\title{
Preparation of Thiazole-2-thiones through TBPB-Promoted Oxidative Cascade Cyclization of Enaminones with Elemental Sulfur
}

Biao Zhang, Donghan Liu, Yulin Sun, Yajing Zhang, Jiayi Feng, and Fuchao Yu*

Faculty of Life Science and Technology, Kunming University of Science and Technology, Kunming, 650504, P. R. China.

\section{Supporting Information}

\section{Table of Contents}

1. General information.

2. General procedure

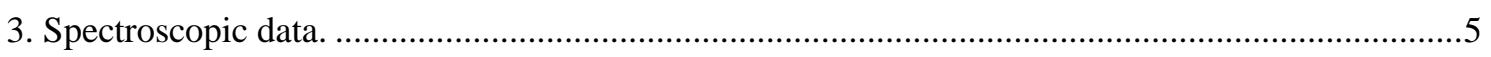

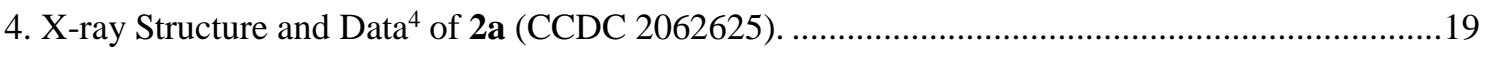

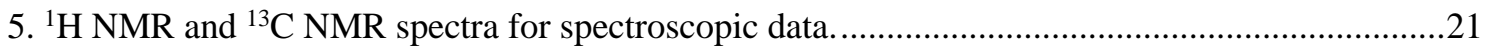

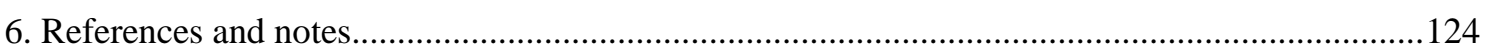




\section{General information.}

All compounds were fully characterized by spectroscopic data. The NMR spectra were recorded on a DRX600 $\left({ }^{1} \mathrm{H}: 600 \mathrm{MHz},{ }^{13} \mathrm{C}: 150 \mathrm{MHz}\right)$, chemical shifts $(\delta)$ are expressed in ppm, and $J$ values are given in $\mathrm{Hz}$, and deuterated $\mathrm{CDCl}_{3}$ and DMSO- $d_{6}$ were used as solvent. The reactions were monitored by thin layer chromatography (TLC) using silica gel $\mathrm{GF}_{254}$. The melting points were determined on XT-4A melting point apparatus and are uncorrected. HRMs were performed on an Agilent LC/MS TOF instrument.

All chemicals and solvents were used as received without further purification unless otherwise stated. Column chromatography was performed on silica gel (200-300 mesh).

Enaminones $\mathbf{1}$ and $\mathbf{3}$ were prepared according to the literature ${ }^{1}$, enaminones $\mathbf{5}$ were prepared according to the literature ${ }^{2}$. Elemental sulfur and oxidant were purchased from Energy Chemical and Adamas-beta®. 


\section{General procedure.}

\subsection{Synthesis of thiazole-2-thiones 2 and 4.}

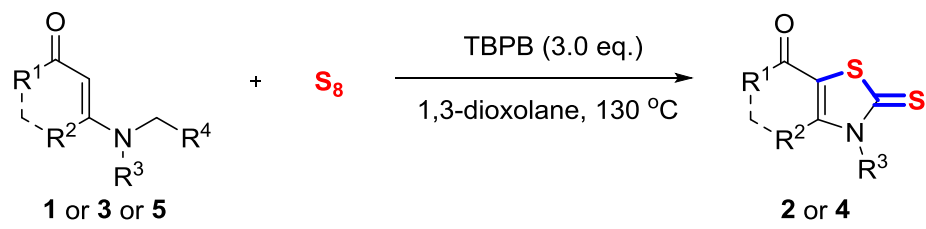

Enaminones 1/3/5 (0.5 mmol), elemental sulfur (1.5 mmol), TBPB (3.0 equiv.) and 1,3-dioxolane $(2.0 \mathrm{~mL})$ were charged into a $10 \mathrm{~mL}$ Ace Glass pressure tubes, and the mixture was stirred at $130{ }^{\circ} \mathrm{C}$ (oil bath) for $3.0 \mathrm{~h}$ until enaminones were completely consumed. The mixture was cooled to room temperature, and then EtOAc $(15 \mathrm{~mL} \times 2)$ were added. The organic phase was washed with water $(10 \mathrm{~mL})$, dried over $\mathrm{Na}_{2} \mathrm{SO}_{4}$, concentrated and purified by flash column chromatography to afford thiazolethiones $\mathbf{2 / 4}$.

\subsection{Synthesis of benzothiazolethiones 7 .}

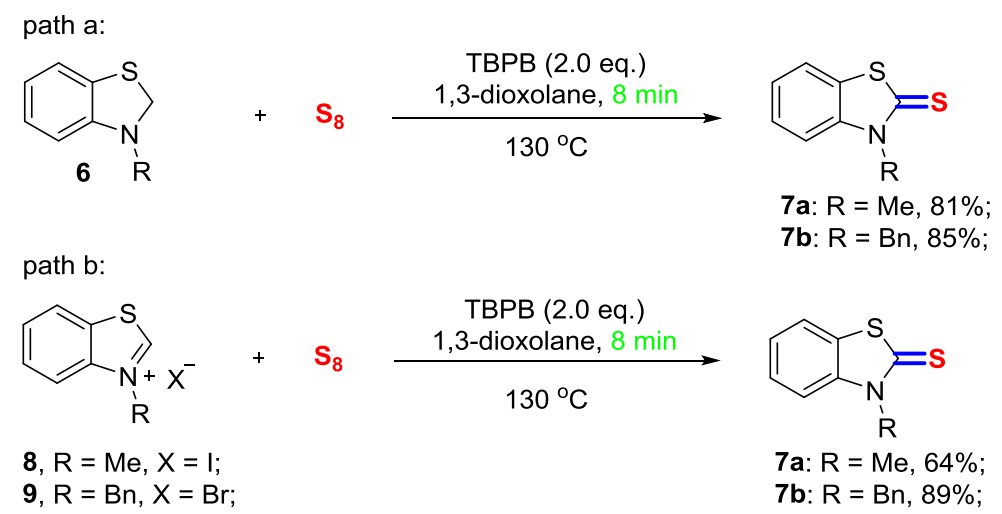

Path a: 2,3-Dihydrobenzo[d]thiazole 6 ( $0.5 \mathrm{mmol})$, elemental sulfur (1.5 mmol), TBPB (2.0 equiv.) and 1,3-dioxolane $(2.0 \mathrm{~mL})$ were charged into a $10 \mathrm{~mL}$ Ace Glass pressure tubes, and the mixture was stirred at $130{ }^{\circ} \mathrm{C}$ (oil bath) for 8 min until enaminones were completely consumed. The mixture was cooled to room temperature, and then EtOAc $(15 \mathrm{~mL} \times 2)$ were added. The organic phase was washed with water $(10 \mathrm{~mL})$, dried over $\mathrm{Na}_{2} \mathrm{SO}_{4}$, concentrated and purified by flash column chromatography to afford benzothiazolethiones 7 .

Path b: Benzo[d]thiazol-3-ium 8/9 (0.5 mmol), elemental sulfur (1.5 mmol), TBPB (2.0 equiv.) and 1,3-dioxolane $(2.0 \mathrm{~mL})$ were charged into a $10 \mathrm{~mL}$ Ace Glass pressure tubes, and the mixture was stirred at $130{ }^{\circ} \mathrm{C}$ (oil bath) for $8 \mathrm{~min}$ until enaminones were completely consumed. The mixture was cooled to room temperature, and then EtOAc $(15 \mathrm{~mL} \times 2)$ were added. The organic phase was washed with water $(10 \mathrm{~mL})$, dried over $\mathrm{Na}_{2} \mathrm{SO}_{4}$, concentrated and purified by flash column chromatography to afford benzothiazolethiones 7. 


\subsection{Scale-up synthesis of thiazole-2-thione 4q.}
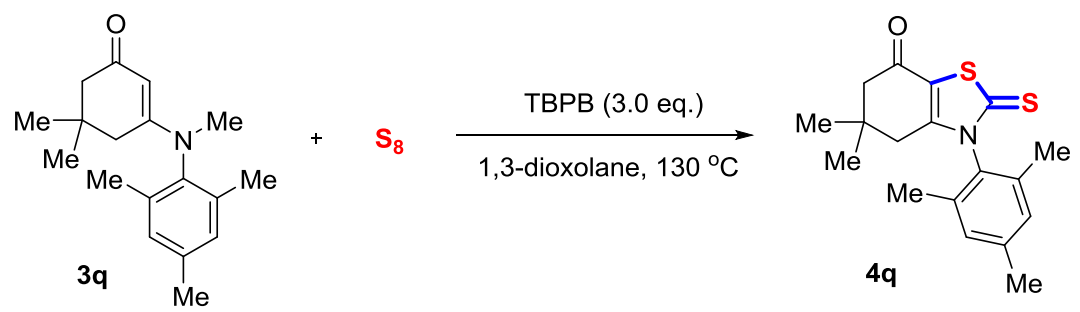

Enaminones 3q (5.8 mmol), elemental sulfur $(17.4 \mathrm{mmol})$, TBPB $(17.4 \mathrm{mmol})$ and 1,3-dioxolane $(23.0 \mathrm{~mL})$ were charged into a $75 \mathrm{~mL}$ Ace Glass pressure tubes, and the mixture was stirred at 130 ${ }^{\circ} \mathrm{C}$ (oil bath) for $3.0 \mathrm{~h}$ until enaminones were completely consumed. The mixture was cooled to room temperature, and then EtOAc $(50 \mathrm{~mL} \times 3)$ were added. The organic phase was washed with water $(50 \mathrm{~mL})$, dried over $\mathrm{Na}_{2} \mathrm{SO}_{4}$, concentrated and purified by flash column chromatography to afford thiazolethiones $\mathbf{4 q}$ in $65 \%$ yield $(1.25 \mathrm{~g})$.

\subsection{Synthesis of thiazole-2-thiones from in situ prepared enamines.}
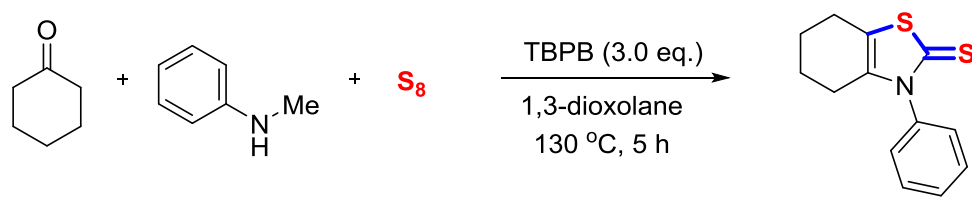

not detected

The reaction of elemental sulfur with in situ prepared enamines from cyclohexanone and $N$-methylaniline were carried out. However, the reaction mixture was too complex and the corresponding target product was not observed.
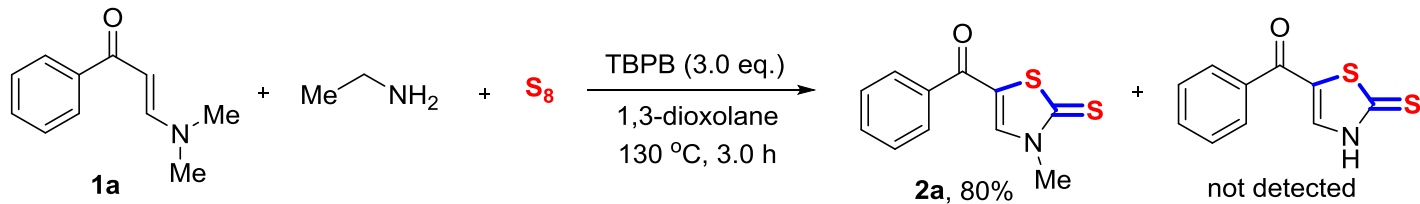

The reaction of enaminones 1a with elemental sulfur was carried out in the additional presence of ethyl amine under the optimized conditions, and the thiazole-2-thione product $2 \mathbf{a}$ was obtained in $80 \%$ yield, but the $\mathrm{NH}$-thiazole-2-thione product was not observed. Therefore, an in situ transamination did not achieved. 


\section{Spectroscopic data.}

(3-Methyl-2-thioxo-2,3-dihydrothiazol-5-yl)(phenyl)methanone (2a)

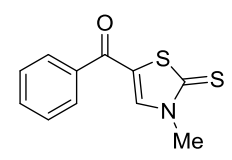

$\mathrm{V}_{\text {Petroleum ether }} / \mathrm{V}_{\text {Ethyl acetate }}=3: 1, \mathrm{R}_{f}=0.20$; Yellow solid: $100 \mathrm{mg}(85 \%) ; \mathrm{mp}=179-181{ }^{\circ} \mathrm{C} ;{ }^{1} \mathrm{H}$ NMR $\left(600 \mathrm{MHz}, \mathrm{CDCl}_{3}\right): \delta=7.70(\mathrm{~d}, J=7.3 \mathrm{~Hz}, 2 \mathrm{H}, \mathrm{ArH}), 7.55-7.58(\mathrm{~m}, 2 \mathrm{H}, \mathrm{C}=\mathrm{CHN}+\mathrm{ArH}), 7.45(\mathrm{t}, J=$ $7.7 \mathrm{~Hz}, 2 \mathrm{H}, \mathrm{ArH}), 3.66$ (s, 3H, $\left.\mathrm{NCH}_{3}\right) ;{ }^{13} \mathrm{C} \mathrm{NMR}\left(150 \mathrm{MHz}, \mathrm{CDCl}_{3}\right): \delta=190.6,184.2,139.1,136.8$, 133.1, 129.0, 129.0, 128.5, 128.5, 126.5, 38.1; HRMS (TOF ES+): $\mathrm{m} / \mathrm{z}$ calcd for $\mathrm{C}_{11} \mathrm{H}_{9} \mathrm{NOS}_{2} \mathrm{Na}$ $\left[(\mathrm{M}+\mathrm{Na})^{+}\right], 258.0018$, found, 258.0020 .

(3-Methyl-2-thioxo-2,3-dihydrothiazol-5-yl)(p-tolyl)methanone (2b)<smiles>Cc1ccc(C(=O)c2cn(-c3ccc(C)cc3)sc2=O)cc1</smiles>

$\mathrm{V}_{\text {Petroleum ether }} / \mathrm{V}_{\text {Ethyl acetate }}=3: 1, \mathrm{R}_{f}=0.20$; Yellow solid: $106 \mathrm{mg}(85 \%) ; \mathrm{mp}=181-182{ }^{\circ} \mathrm{C} ;{ }^{1} \mathrm{H}$ NMR $\left(600 \mathrm{MHz}, \mathrm{CDCl}_{3}\right): \delta=7.68(\mathrm{~d}, J=8.1 \mathrm{~Hz}, 2 \mathrm{H}, \mathrm{ArH}), 7.62(\mathrm{~s}, 1 \mathrm{H}, \mathrm{C}=\mathrm{CHN}), 7.32(\mathrm{~d}, J=7.9 \mathrm{~Hz}, 2 \mathrm{H}$, $\mathrm{ArH}), 3.72$ (s, 3H, $\mathrm{NCH}_{3}$ ), 2.44 (s, 3H, $\left.\mathrm{ArCH}_{3}\right) ;{ }^{13} \mathrm{C} \mathrm{NMR} \mathrm{(150} \mathrm{MHz,} \mathrm{CDCl}_{3}$ ): $\delta=190.5,183.9$, 144.1, 138.7, 134.1, 129.6, 129.6, 128.7, 128.7, 126.6, 38.1, 21.7; HRMS (TOF ES+): m/z calcd for $\mathrm{C}_{12} \mathrm{H}_{12} \mathrm{NOS}_{2}\left[(\mathrm{M}+\mathrm{H})^{+}\right], 250.0355$, found, 250.0350 .

(4-Methoxyphenyl)(3-methyl-2-thioxo-2,3-dihydrothiazol-5-yl)methanone (2c)

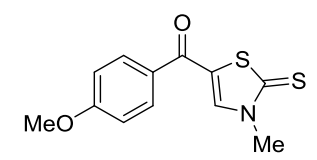

$\mathrm{V}_{\text {Petroleum ether }} / \mathrm{V}_{\text {Ethyl acetate }}=5: 1, \mathrm{R}_{f}=0.10$; Yellow solid: $117 \mathrm{mg}(88 \%) ; \mathrm{mp}=167-168{ }^{\circ} \mathrm{C} ;{ }^{1} \mathrm{H}$ NMR $\left(600 \mathrm{MHz}, \mathrm{CDCl}_{3}\right): \delta=7.82(\mathrm{~d}, J=8.7 \mathrm{~Hz}, 2 \mathrm{H}, \mathrm{ArH}), 7.65(\mathrm{~s}, 1 \mathrm{H}, \mathrm{C}=\mathrm{CHN}), 7.02(\mathrm{~d}, J=8.7 \mathrm{~Hz}, 2 \mathrm{H}$, $\mathrm{ArH}), 3.92$ (s, $\left.3 \mathrm{H}, \mathrm{ArOCH}_{3}\right), 3.76$ (s, $\left.3 \mathrm{H}, \mathrm{NCH}_{3}\right) ;{ }^{13} \mathrm{C} \mathrm{NMR}\left(150 \mathrm{MHz}, \mathrm{CDCl}_{3}\right): \delta=190.4,182.7$, 163.7, 138.2, 131.0, 131.0, 129.4, 126.6, 114.2, 114.2, 55.7, 38.1; HRMS (TOF ES+): m/z calcd for $\mathrm{C}_{12} \mathrm{H}_{12} \mathrm{NO}_{2} \mathrm{~S}_{2}\left[(\mathrm{M}+\mathrm{H})^{+}\right], 266.0304$, found, 266.0300 .

(4-Fluorophenyl)(3-methyl-2-thioxo-2,3-dihydrothiazol-5-yl)methanone (2d)

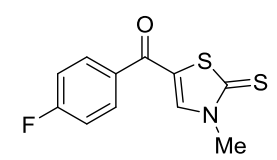

$\mathrm{V}_{\text {Petroleum ether }} / \mathrm{V}_{\text {Ethyl acetate }}=3: 1, \mathrm{R}_{f}=0.20$; Yellow solid: $104 \mathrm{mg}(82 \%) ; \mathrm{mp}=175-177{ }^{\circ} \mathrm{C} ;{ }^{1} \mathrm{H}$ NMR (600 MHz, $\left.\mathrm{CDCl}_{3}\right): \delta=7.83-7.85(\mathrm{~m}, 2 \mathrm{H}, \mathrm{ArH}), 7.64(\mathrm{~s}, 1 \mathrm{H}, \mathrm{C}=\mathrm{CHN}), 7.21-7.24(\mathrm{~m}, 2 \mathrm{H}, \mathrm{ArH})$, $3.76\left(\mathrm{~s}, 1 \mathrm{H}, \mathrm{NCH}_{3}\right) ;{ }^{13} \mathrm{C} \mathrm{NMR}\left(150 \mathrm{MHz}, \mathrm{CDCl}_{3}\right): \delta=190.6,182.7,166.5(\mathrm{C}-\mathrm{F}, 1 \mathrm{JC}-\mathrm{F}=255.6 \mathrm{~Hz})$, $164.8(\mathrm{C}-\mathrm{F}, 1 \mathrm{JC}-\mathrm{F}=255.6 \mathrm{~Hz}), 138.8,133.1,131.1,131.2(\mathrm{C}-\mathrm{F}, 3 \mathrm{JC}-\mathrm{F}=9.1 \mathrm{~Hz}), 131.1(\mathrm{C}-\mathrm{F}, 3 \mathrm{JC}-\mathrm{F}$ = 9.1 Hz), 126.1, $116.3(\mathrm{C}-\mathrm{F}, 2 \mathrm{JC}-\mathrm{F}=21.9 \mathrm{~Hz}), 116.1(\mathrm{C}-\mathrm{F}, 2 \mathrm{JC}-\mathrm{F}=21.9 \mathrm{~Hz}), 38.1$; HRMS (TOF $\mathrm{ES}+): \mathrm{m} / \mathrm{z}$ calcd for $\mathrm{C}_{11} \mathrm{H}_{9} \mathrm{FNOS}_{2}\left[(\mathrm{M}+\mathrm{H})^{+}\right], 254.0104$, found, 254.0098 .

13C NMR (150 MHz, CDCl3): (ppm) 168.3, 166.7, 161.7 (C-F, 1JC-F = 249.0 Hz), 160.0 (C-F, $1 \mathrm{JC}-\mathrm{F}=249.0 \mathrm{~Hz}), 150.2,144.1,139.6,135.7,130.6(\mathrm{C}-\mathrm{F}, 3 \mathrm{JC}-\mathrm{F}=9.0 \mathrm{~Hz}), 130.5(\mathrm{C}-\mathrm{F}, 3 \mathrm{JC}-\mathrm{F}=$ 
9.0 Hz), 128.7, 127.6, 127.4, 127.3, 126.0, 121.6, 121.4, 111.3 (C-F, 2JC-F = 22.5 Hz), 111.1 (C-F, $2 \mathrm{JC}-\mathrm{F}=22.5 \mathrm{~Hz}), 59.3,50.2,48.9,41.9,32.7,29.7,25.4,24.7,24.6$;

(4-Chlorophenyl)(3-methyl-2-thioxo-2,3-dihydrothiazol-5-yl)methanone (2e)<smiles>O=C(c1ccc(Cl)cc1)c1cn(S)c(=O)s1</smiles>

$\mathrm{V}_{\text {Petroleum ether }} / \mathrm{V}_{\text {Ethyl acetate }}=2: 1, \mathrm{R}_{f}=0.30$; Yellow solid: $112 \mathrm{mg}(83 \%) ; \mathrm{mp}=193-195{ }^{\circ} \mathrm{C} ;{ }^{1} \mathrm{H}$ NMR $\left(600 \mathrm{MHz}, \mathrm{CDCl}_{3}\right): \delta=7.66(\mathrm{~d}, J=8.5 \mathrm{~Hz}, 2 \mathrm{H}, \mathrm{ArH}), 7.55(\mathrm{~s}, 1 \mathrm{H}, \mathrm{C}=\mathrm{CHN}), 7.44(\mathrm{~d}, J=8.5 \mathrm{~Hz}, 2 \mathrm{H}$, $\mathrm{ArH}), 3.67$ (s, 3H, $\left.\mathrm{NCH}_{3}\right) ;{ }^{13} \mathrm{C} \mathrm{NMR}\left(150 \mathrm{MHz}, \mathrm{CDCl}_{3}\right.$ ): $\delta=190.6,182.9,139.6,139.0,135.1$, 129.9, 129.9, 129.3, 129.3, 126.0, 38.1; HRMS (TOF ES+): $\mathrm{m} / \mathrm{z}$ calcd for $\mathrm{C}_{11} \mathrm{H}_{9} \mathrm{ClNOS}_{2}\left[(\mathrm{M}+\mathrm{H})^{+}\right]$, 269.9809, found, 269.9803.

(4-Bromophenyl)(3-methyl-2-thioxo-2,3-dihydrothiazol-5-yl)methanone (2f)

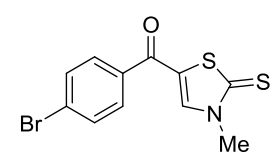

$\mathrm{V}_{\text {Petroleum ether }} / \mathrm{V}_{\text {Ethyl acetate }}=4: 1, \mathrm{R}_{f}=0.20$; Yellow solid: $127 \mathrm{mg}(81 \%) ; \mathrm{mp}=172-174{ }^{\circ} \mathrm{C} ;{ }^{1} \mathrm{H}$ NMR $\left(600 \mathrm{MHz}, \mathrm{CDCl}_{3}\right): \delta=7.63-7.67(\mathrm{~m}, 4 \mathrm{H}, \mathrm{ArH}), 7.61(\mathrm{~s}, 1 \mathrm{H}, \mathrm{C}=\mathrm{CHN}), 3.73\left(\mathrm{~s}, 3 \mathrm{H}, \mathrm{NCH}_{3}\right) ;{ }^{13} \mathrm{C}$ $\operatorname{NMR}\left(150 \mathrm{MHz}, \mathrm{CDCl}_{3}\right): \delta=190.6,183.0,139.1,135.5,132.3,132.3,130.0,130.0,128.1,126.0$, 38.1; HRMS (TOF ES+): $\mathrm{m} / \mathrm{z}$ calcd for $\mathrm{C}_{11} \mathrm{H}_{9} \mathrm{BrNOS}_{2}\left[(\mathrm{M}+\mathrm{H})^{+}\right]$, 313.9303, found, 313.9295 .

(2-Chlorophenyl)(3-methyl-2-thioxo-2,3-dihydrothiazol-5-yl)methanone (2g)

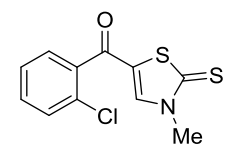

$\mathrm{V}_{\text {Petroleum ether }} / \mathrm{V}_{\text {Ethyl acetate }}=3: 1, \mathrm{R}_{f}=0.30$; Yellow solid: $105 \mathrm{mg}(78 \%) ; \mathrm{mp}=117-119^{\circ} \mathrm{C} ;{ }^{1} \mathrm{H}$ NMR $\left(600 \mathrm{MHz}, \mathrm{CDCl}_{3}\right): \delta=7.48-7.52(\mathrm{~m}, 2 \mathrm{H}, \mathrm{ArH}), 7.40-7.43(\mathrm{~m}, 2 \mathrm{H}, \mathrm{ArH}), 7.36(\mathrm{~s}, 1 \mathrm{H}, \mathrm{C}=\mathrm{CHN})$, $3.69\left(\mathrm{~s}, 1 \mathrm{H}, \mathrm{NCH}_{3}\right) ;{ }^{13} \mathrm{C}$ NMR $\left(150 \mathrm{MHz}, \mathrm{CDCl}_{3}\right): \delta=191.1,183.1,140.4,136.7,132.0,131.2$, 130.6, 128.8, 127.0, 126.4, 38.1; HRMS (TOF ES+): $\mathrm{m} / \mathrm{z}$ calcd for $\mathrm{C}_{11} \mathrm{H}_{9} \mathrm{ClNOS}_{2}\left[(\mathrm{M}+\mathrm{H})^{+}\right]$, 269.9809 found, 269.9802 .

(2,4-Dichlorophenyl)(3-methyl-2-thioxo-2,3-dihydrothiazol-5-yl)methanone (2h)

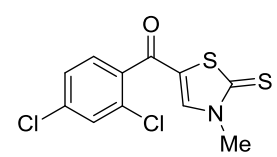

$\mathrm{V}_{\text {Petroleum ether }} / \mathrm{V}_{\text {Ethyl acetate }}=3: 1, \mathrm{R}_{f}=0.20$; Yellow solid: $126 \mathrm{mg}(83 \%) ; \mathrm{mp}=156-157{ }^{\circ} \mathrm{C} ;{ }^{1} \mathrm{H}$ NMR $\left(600 \mathrm{MHz}, \mathrm{CDCl}_{3}\right): \delta=7.54(\mathrm{~d}, J=1.8 \mathrm{~Hz}, 1 \mathrm{H}, \mathrm{ArH}), 7.40(\mathrm{~d}, J=1.8 \mathrm{~Hz}, 1 \mathrm{H}, \operatorname{ArH}), 7.39(\mathrm{~s}, 1 \mathrm{H}$, $\mathrm{C}=\mathrm{CHN}), 7.38(\mathrm{~s}, 1 \mathrm{H}, \mathrm{ArH}), 3.70\left(\mathrm{~s}, 3 \mathrm{H}, \mathrm{NCH}_{3}\right) ;{ }^{13} \mathrm{C} \mathrm{NMR}\left(150 \mathrm{MHz}, \mathrm{CDCl}_{3}\right): \delta=191.0,182.0$, 140.4, 137.7, 135.0, 132.3, 130.6, 129.7, 127.5, 126.1, 38.1; HRMS (TOF ES+): $\mathrm{m} / \mathrm{z}$ calcd for $\mathrm{C}_{11} \mathrm{H}_{8} \mathrm{Cl}_{2} \mathrm{NOS}_{2}\left[(\mathrm{M}+\mathrm{H})^{+}\right], 303.9419$, found, 303.9413 .

(3-Methyl-2-thioxo-2,3-dihydrothiazol-5-yl)(4-nitrophenyl)methanone (2i) 


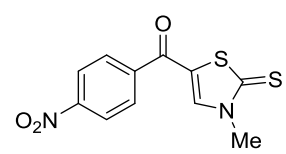

$\mathrm{V}_{\text {Petroleum ether }} / \mathrm{V}_{\text {Ethyl acetate }}=1: 1, \mathrm{R}_{f}=0.20$; Yellow solid: $52 \mathrm{mg}(37 \%) ; \mathrm{mp}=204-206{ }^{\circ} \mathrm{C} ;{ }^{1} \mathrm{H}$ NMR $\left(600 \mathrm{MHz}, \mathrm{CDCl}_{3}\right): \delta=8.40$ (s, 2H, ArH), $7.94(\mathrm{~s}, 2 \mathrm{H}, \mathrm{ArH}), 7.64(\mathrm{~s}, 1 \mathrm{H}, \mathrm{C}=\mathrm{CHN}), 3.76(\mathrm{~s}, 3 \mathrm{H}$, $\left.\mathrm{NCH}_{3}\right) ;{ }^{13} \mathrm{C} \mathrm{NMR}\left(150 \mathrm{MHz}, \mathrm{CDCl}_{3}\right): \delta=190.7,182.2,150.1,141.9,139.9,129.5,129.5,125.4$, 124.2, 124.2, 38.2; HRMS (TOF ES+): $\mathrm{m} / \mathrm{z}$ calcd for $\mathrm{C}_{11} \mathrm{H}_{9} \mathrm{~N}_{2} \mathrm{O}_{3} \mathrm{~S}_{2}\left[(\mathrm{M}+\mathrm{H})^{+}\right], 281.0049$, found, 281.0042 .

4-(3-Methyl-2-thioxo-2,3-dihydrothiazole-5-carbonyl)benzonitrile (2j)

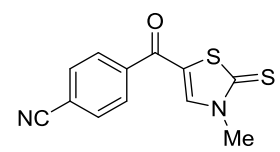

$\mathrm{V}_{\text {Petroleum ether }} / \mathrm{V}_{\text {Ethyl acetate }}=1: 1, \mathrm{R}_{f}=0.20$; Yellow solid: $85 \mathrm{mg}(65 \%) ; \mathrm{mp}=212-213{ }^{\circ} \mathrm{C} ;{ }^{1} \mathrm{H}$ NMR $\left(600 \mathrm{MHz}, \mathrm{CDCl}_{3}\right): \delta=7.85-7.89(\mathrm{~m}, 4 \mathrm{H}, \mathrm{ArH}), 7.63(\mathrm{~s}, 1 \mathrm{H}, \mathrm{C}=\mathrm{CHN}), 3.76\left(\mathrm{~s}, 3 \mathrm{H}, \mathrm{NCH}_{3}\right) ;{ }^{13} \mathrm{C}$ $\operatorname{NMR}\left(150 \mathrm{MHz}, \mathrm{CDCl}_{3}\right): \delta=190.7,182.5,140.3,139.7,132.8,132.8,128.9,128.9,125.4,117.6$, 116.4, 38.2; HRMS (TOF ES+): $\mathrm{m} / \mathrm{z}$ calcd for $\mathrm{C}_{12} \mathrm{H}_{9} \mathrm{~N}_{2} \mathrm{OS}_{2}\left[(\mathrm{M}+\mathrm{H})^{+}\right]$, 261.0151, found,261.0149.

(3-Methyl-2-thioxo-2,3-dihydrothiazol-5-yl)(4-(methylsulfonyl)phenyl)methanone (2k)

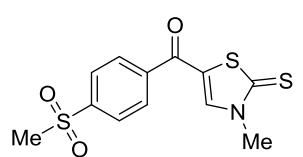

$\mathrm{V}_{\text {Petroleum ether }} / \mathrm{V}_{\text {Ethyl acetate }}=1: 1, \mathrm{R}_{f}=0.20$; Yellow solid: $105 \mathrm{mg}(67 \%) ; \mathrm{mp}=197-199{ }^{\circ} \mathrm{C} ;{ }^{1} \mathrm{H}$ NMR $\left(600 \mathrm{MHz}, \mathrm{CDCl}_{3}\right): \delta=8.11(\mathrm{~d}, J=8.2 \mathrm{~Hz}, 2 \mathrm{H}, \mathrm{ArH}), 7.94(\mathrm{~d}, J=8.2 \mathrm{~Hz}, 2 \mathrm{H}, \mathrm{ArH}), 7.61(\mathrm{~s}, 1 \mathrm{H}$, $\mathrm{C}=\mathrm{CHN}), 3.73\left(\mathrm{~s}, 3 \mathrm{H}, \mathrm{NCH}_{3}\right), 3.12\left(\mathrm{~s}, 3 \mathrm{H}, \mathrm{SCH}_{3}\right) ;{ }^{13} \mathrm{C} \mathrm{NMR}\left(150 \mathrm{MHz}, \mathrm{CDCl}_{3}\right): \delta=190.8,182.7$, 144.1, 141.4, 140.0, 129.3, 129.3, 128.1, 128.1, 125.5, 44.4, 38.2; HRMS (TOF ES+): m/z calcd for $\mathrm{C}_{12} \mathrm{H}_{12} \mathrm{NO}_{3} \mathrm{~S}_{3}\left[(\mathrm{M}+\mathrm{H})^{+}\right]$, 313.9974, found, 313.9979.

(3-Methyl-2-thioxo-2,3-dihydrothiazol-5-yl)(4-(trifluoromethyl)phenyl)methanone (2l)

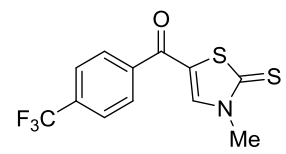

$\mathrm{V}_{\text {Petroleum ether }} / \mathrm{V}_{\text {Ethyl acetate }}=3: 1, \mathrm{R}_{f}=0.30$; Yellow solid: $106 \mathrm{mg}(70 \%) ; \mathrm{mp}=127-129{ }^{\circ} \mathrm{C} ;{ }^{1} \mathrm{H}$ NMR $\left(600 \mathrm{MHz}, \mathrm{CDCl}_{3}\right): \delta=7.90(\mathrm{~d}, J=8.0 \mathrm{~Hz}, 2 \mathrm{H}, \mathrm{ArH}), 7.82(\mathrm{~d}, J=8.0 \mathrm{~Hz}, 2 \mathrm{H}, \operatorname{ArH}), 7.63(\mathrm{~s}, 1 \mathrm{H}$, $\mathrm{C}=\mathrm{CHN}), 3.76\left(\mathrm{~s}, 3 \mathrm{H}, \mathrm{NCH}_{3}\right) ;{ }^{13} \mathrm{C} \mathrm{NMR}\left(150 \mathrm{MHz}, \mathrm{CDCl}_{3}\right): \delta=190.7,183.0,139.8,139.6,134.4$ (q, $J=37.6 \mathrm{~Hz}), 128.8,128.8,126.1(\mathrm{q}, J=3.7 \mathrm{~Hz}), 126.1(\mathrm{q}, J=3.7 \mathrm{~Hz}), 125.8,123.4(\mathrm{q}, J=272.3$ $\mathrm{Hz}$ ), 38.2; HRMS (TOF ES+): m/z calcd for $\mathrm{C}_{12} \mathrm{H}_{9} \mathrm{~F}_{3} \mathrm{NOS}_{2}\left[(\mathrm{M}+\mathrm{H})^{+}\right], 304.0072$, found, 304.0070.

(4-(Dimethylamino)phenyl)(3-methyl-2-thioxo-2,3-dihydrothiazol-5-yl)methanone (2m)

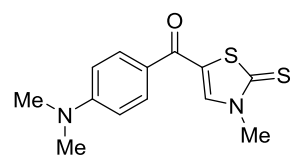

$\mathrm{V}_{\text {Petroleum ether }} / \mathrm{V}_{\text {Ethyl acetate }}=1: 1, \mathrm{R}_{f}=0.20$; Yellow solid: $77 \mathrm{mg}(55 \%) ; \mathrm{mp}=193-195{ }^{\circ} \mathrm{C} ;{ }^{1} \mathrm{H}$ NMR $\left(600 \mathrm{MHz}, \mathrm{CDCl}_{3}\right): \delta=7.82(\mathrm{~d}, J=8.7 \mathrm{~Hz}, 2 \mathrm{H}, \mathrm{ArH}), 7.66(\mathrm{~s}, 1 \mathrm{H}, \mathrm{C}=\mathrm{CHN}), 6.87(\mathrm{~d}, J=8.4 \mathrm{~Hz}, 2 \mathrm{H}$, 
$\mathrm{ArH}), 3.76$ (s, 3H, $\left.\mathrm{NCH}_{3}\right), 3.13\left(\mathrm{~s}, 6 \mathrm{H}, \mathrm{NCH}_{3}\right) ;{ }^{13} \mathrm{C} \mathrm{NMR}\left(150 \mathrm{MHz}, \mathrm{CDCl}_{3}\right): \delta=190.0,181.8$, 152.8, 137.4, 131.2, 131.2, 131.2, 131.2, 127.0, 112.3, 40.9, 38.0; HRMS (TOF ES+): $\mathrm{m} / \mathrm{z}$ calcd for $\mathrm{C}_{13} \mathrm{H}_{15} \mathrm{~N}_{2} \mathrm{OS}_{2}\left[(\mathrm{M}+\mathrm{H})^{+}\right], 279.0620$, found, 279.0620 .

$N$-(4-(3-Methyl-2-thioxo-2,3-dihydrothiazole-5-carbonyl)phenyl)acetamide (2n)

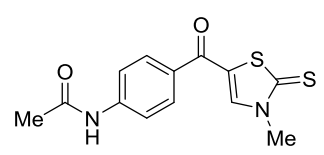

$\mathrm{V}_{\text {Petroleum ether }} / \mathrm{V}_{\text {Ethyl acetate }}=1: 1, \mathrm{R}_{f}=0.20$; Yellow solid: $85 \mathrm{mg}(58 \%) ; \mathrm{mp}=264-266{ }^{\circ} \mathrm{C} ;{ }^{1} \mathrm{H}$ NMR (600 MHz, DMSO- $\left.d_{6}\right): \delta=10.37(\mathrm{~s}, 1 \mathrm{H}, \mathrm{NH}), 8.44(\mathrm{~s}, 1 \mathrm{H}, \mathrm{C}=\mathrm{CHN}), 7.86(\mathrm{~d}, J=8.7 \mathrm{~Hz}, 2 \mathrm{H}, \mathrm{ArH})$, $7.78(\mathrm{~d}, J=8.7 \mathrm{~Hz}, 2 \mathrm{H}, \mathrm{ArH}), 3.66\left(\mathrm{~s}, 3 \mathrm{H}, \mathrm{NCH}_{3}\right), 2.11\left(\mathrm{~s}, 3 \mathrm{H}, \mathrm{CCH}_{3}\right) ;{ }^{13} \mathrm{C} \mathrm{NMR}(150 \mathrm{MHz}$, DMSO- $\left.d_{6}\right): \delta=189.6,182.9,169.6,144.2,142.4,130.8,130.7,130.7,125.3,118.9,118.9,38.2$, 24.7; HRMS (TOF ES+): $\mathrm{m} / \mathrm{z}$ calcd for $\mathrm{C}_{13} \mathrm{H}_{13} \mathrm{~N}_{2} \mathrm{O}_{2} \mathrm{~S}_{2}\left[(\mathrm{M}+\mathrm{H})^{+}\right]$, 293.0413, found, 293.0409.

\section{(2-Hydroxyphenyl)(3-methyl-2-thioxo-2,3-dihydrothiazol-5-yl)methanone (2o)}

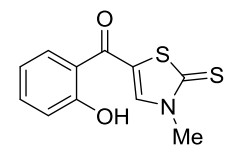

$\mathrm{V}_{\text {Petroleum ether }} / \mathrm{V}_{\text {Ethyl acetate }}=3: 1, \mathrm{R}_{f}=0.30$; Yellow solid: $92 \mathrm{mg}(73 \%) ; \mathrm{mp}=144-145{ }^{\circ} \mathrm{C} ;{ }^{1} \mathrm{H}$ NMR $\left(600 \mathrm{MHz}, \mathrm{CDCl}_{3}\right): \delta=11.13(\mathrm{~s}, 1 \mathrm{H}, \mathrm{OH}), 7.71(\mathrm{~s}, 1 \mathrm{H}, \mathrm{C}=\mathrm{CHN}), 7.68-7.70(\mathrm{~m}, 1 \mathrm{H}, \mathrm{ArH})$, 7.47-7.50 (m, 1H, ArH), 7.01 (d, $J=8.4 \mathrm{~Hz}, 1 \mathrm{H}, \mathrm{ArH}), 6.91(\mathrm{t}, J=7.6 \mathrm{~Hz}, 1 \mathrm{H}, \operatorname{ArH}), 3.70$ (s, 3H, $\left.\mathrm{NCH}_{3}\right) ;{ }^{13} \mathrm{C}$ NMR $\left(150 \mathrm{MHz}, \mathrm{CDCl}_{3}\right): \delta=190.1,186.4,162.3,138.8,136.7,129.9,124.8,119.5$, 119.0, 118.8, 38.1; HRMS (TOF ES+): $\mathrm{m} / \mathrm{z}$ calcd for $\mathrm{C}_{11} \mathrm{H}_{10} \mathrm{NO}_{2} \mathrm{~S}_{2}\left[(\mathrm{M}+\mathrm{H})^{+}\right], 252.0147$, found, 252.0144 .

(2-Hydroxy-5-methoxyphenyl)(3-methyl-2-thioxo-2,3-dihydrothiazol-5-yl)methanone (2p)

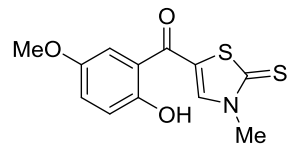

$\mathrm{V}_{\text {Petroleum ether }} / \mathrm{V}_{\text {Ethyl acetate }}=2: 1, \mathrm{R}_{f}=0.10$; Yellow solid: $105 \mathrm{mg}(75 \%) ; \mathrm{mp}=152-153{ }^{\circ} \mathrm{C} ;{ }^{1} \mathrm{H}$ NMR (600 MHz, $\left.\mathrm{CDCl}_{3}\right): \delta=12.03(\mathrm{~s}, 1 \mathrm{H}, \mathrm{OH}), 7.74(\mathrm{~s}, 1 \mathrm{H}, \mathrm{C}=\mathrm{CHN}), 7.71(\mathrm{~d}, J=9.3 \mathrm{~Hz}, 1 \mathrm{H}, \mathrm{ArH})$, $6.53(\mathrm{~d}, J=7.8 \mathrm{~Hz}, 2 \mathrm{H}, \mathrm{ArH}), 3.89\left(\mathrm{~s}, 3 \mathrm{H}, \mathrm{OCH}_{3}\right), 3.78\left(\mathrm{~s}, 3 \mathrm{H}, \mathrm{NCH}_{3}\right) ;{ }^{13} \mathrm{C} \mathrm{NMR}(150 \mathrm{MHz}$, $\left.\mathrm{CDCl}_{3}\right): \delta=189.7,184.8,166.7,165.9,137.7,131.7,124.8,112.5,108.5,101.5,55.8,38.1 ;$ HRMS (TOF ES+): $\mathrm{m} / \mathrm{z}$ calcd for $\mathrm{C}_{12} \mathrm{H}_{12} \mathrm{NO}_{3} \mathrm{~S}_{2}\left[(\mathrm{M}+\mathrm{H})^{+}\right], 282.0253$, found, 282.0256 .

(5-Chloro-2-hydroxyphenyl)(3-methyl-2-thioxo-2,3-dihydrothiazol-5-yl)methanone (2q)<smiles>Nn1cc(C(=O)Sc2cc(Cl)ccc2O)sc1=S</smiles>

$\mathrm{V}_{\text {Petroleum ether }} / \mathrm{V}_{\text {Ethyl acetate }}=2: 1, \mathrm{R}_{f}=0.20$; Yellow solid: $101 \mathrm{mg}(71 \%) ; \mathrm{mp}=153-155{ }^{\circ} \mathrm{C} ;{ }^{1} \mathrm{H}$ NMR $\left(600 \mathrm{MHz}, \mathrm{CDCl}_{3}\right): \delta=11.08(\mathrm{~s}, 1 \mathrm{H}, \mathrm{OH}), 7.81(\mathrm{~s}, 1 \mathrm{H}, \mathrm{C}=\mathrm{CHN}), 7.73(\mathrm{~s}, 1 \mathrm{H}, \mathrm{ArH}), 7.51(\mathrm{dd}, J=$ 8.9, $2.5 \mathrm{~Hz}, 1 \mathrm{H}, \mathrm{ArH}), 7.07$ (d, $J=8.9 \mathrm{~Hz}, 1 \mathrm{H}, \mathrm{ArH}), 3.81\left(\mathrm{~s}, 3 \mathrm{H}, \mathrm{NCH}_{3}\right) ;{ }^{13} \mathrm{C}$ NMR $(150 \mathrm{MHz}$, $\mathrm{CDCl}_{3}$ ): $\delta=190.1,186.3,160.7,139.0,135.5,128.9,128.9,124.3,120.7,119.4,38.3$; HRMS (TOF $\mathrm{ES}+): \mathrm{m} / \mathrm{z}$ calcd for $\mathrm{C}_{11} \mathrm{H}_{9} \mathrm{ClNO}_{2} \mathrm{~S}_{2}\left[(\mathrm{M}+\mathrm{H})^{+}\right], 285.9758$, found, 285.9760 . 
Benzo $[d][1,3]$ dioxol-5-yl(3-methyl-2-thioxo-2,3-dihydrothiazol-5-yl)methanone (2r)

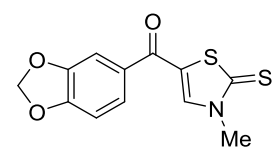

$\mathrm{V}_{\text {Petroleum ether }} / \mathrm{V}_{\text {Ethyl acetate }}=2: 1, \mathrm{R}_{f}=0.20$; Yellow solid: $110 \mathrm{mg}(79 \%) ; \mathrm{mp}=169-171{ }^{\circ} \mathrm{C} ;{ }^{1} \mathrm{H}$ NMR $\left(600 \mathrm{MHz}, \mathrm{CDCl}_{3}\right): \delta=7.66$ (s, 1H, C=CHN), $7.41(\mathrm{~d}, J=7.9 \mathrm{~Hz}, 1 \mathrm{H}, \mathrm{ArH}), 7.29(\mathrm{~s}, 1 \mathrm{H}, \mathrm{ArH}), 6.92$ $(\mathrm{d}, J=8 \mathrm{~Hz}, 1 \mathrm{H}, \mathrm{ArH}), 6.11\left(\mathrm{~s}, 2 \mathrm{H}, \mathrm{OCH}_{2} \mathrm{O}\right), 3.75\left(\mathrm{~s}, 3 \mathrm{H}, \mathrm{NCH}_{3}\right) ;{ }^{13} \mathrm{C} \mathrm{NMR}\left(150 \mathrm{MHz}, \mathrm{CDCl}_{3}\right): \delta=$ 190.4, 182.3 152.1, 148.5, 138.3, 131.0, 125.4, 124.8, 108.7, 108.3, 102.2, 38.1; HRMS (TOF ES+): $\mathrm{m} / \mathrm{z}$ calcd for $\mathrm{C}_{12} \mathrm{H}_{10} \mathrm{NO}_{3} \mathrm{~S}_{2}\left[(\mathrm{M}+\mathrm{H})^{+}\right], 280.0097$, found, 280.0092 .

(3-Methyl-2-thioxo-2,3-dihydrothiazol-5-yl)(naphthalen-2-yl)methanone (2s)

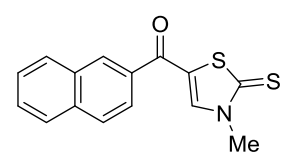

$\mathrm{V}_{\text {Petroleum ether }} / \mathrm{V}_{\text {Ethyl acetate }}=2: 1, \mathrm{R}_{f}=0.30$; Yellow solid: $113 \mathrm{mg}(79 \%) ; \mathrm{mp}=195-196{ }^{\circ} \mathrm{C} ;{ }^{1} \mathrm{H}$ NMR $\left(600 \mathrm{MHz}, \mathrm{CDCl}_{3}\right): \delta=8.29$ (s, $\left.1 \mathrm{H}, \mathrm{C}=\mathrm{CHN}\right), 7.97(\mathrm{dd}, J=8.1,4.8 \mathrm{~Hz}, 2 \mathrm{H}, \operatorname{ArH}), 7.92(\mathrm{~d}, J=8.1$ $\mathrm{Hz}, 1 \mathrm{H}, \mathrm{ArH}), 7.82$ (dd, $J=8.5,1.5 \mathrm{~Hz}, 1 \mathrm{H}, \mathrm{ArH}), 7.69$ (s, 1H, ArH), 7.59-7.66 (m, 2H, ArH), 3.74 (s, $\left.3 \mathrm{H}, \mathrm{NCH}_{3}\right) ;{ }^{13} \mathrm{C} \mathrm{NMR}\left(150 \mathrm{MHz}, \mathrm{CDCl}_{3}\right): \delta=190.6,184.2,139.1,135.4,134.1,132.3,129.8$, 129.3, 129.1, 128.8, 128.0, 127.4, 126.6, 124.5, 38.1; HRMS (TOF ES+): $\mathrm{m} / \mathrm{z}$ calcd for $\mathrm{C}_{15} \mathrm{H}_{12} \mathrm{NOS}_{2}$ $\left[(\mathrm{M}+\mathrm{H})^{+}\right], 286.0355$, found, 286.0352.

\section{[1,1'-Biphenyl]-4-yl(3-methyl-2-thioxo-2,3-dihydrothiazol-5-yl)methanone (2t)}

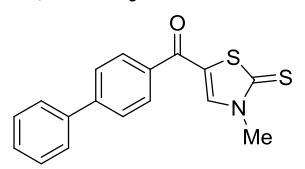

$\mathrm{V}_{\text {Petroleum ether }} / \mathrm{V}_{\text {Ethyl acetate }}=2: 1, \mathrm{R}_{f}=0.30$; Yellow solid: $146 \mathrm{mg}(94 \%) ; \mathrm{mp}=187-189{ }^{\circ} \mathrm{C} ;{ }^{1} \mathrm{H}$ NMR $\left(600 \mathrm{MHz}, \mathrm{CDCl}_{3}\right): \delta=7.89(\mathrm{~d}, J=8.3 \mathrm{~Hz}, 2 \mathrm{H}, \mathrm{ArH}), 7.76(\mathrm{~d}, J=8.4 \mathrm{~Hz}, 2 \mathrm{H}, \mathrm{ArH}), 7.70(\mathrm{~s}, 1 \mathrm{H}$, $\mathrm{C}=\mathrm{CHN}), 7.65-7.67(\mathrm{~m}, 2 \mathrm{H}, \mathrm{ArH}), 7.51-7.53(\mathrm{~m}, 2 \mathrm{H}, \mathrm{ArH}), 7.44-7.47(\mathrm{~m}, 1 \mathrm{H}, \mathrm{ArH}), 3.77(\mathrm{~s}, 3 \mathrm{H}$, $\left.\mathrm{NCH}_{3}\right) ;{ }^{13} \mathrm{C}$ NMR $\left(150 \mathrm{MHz}, \mathrm{CDCl}_{3}\right): \delta=190.6,183.7,146.0,139.5,138.9,135.4,129.2,129.2$, 129.1, 129.1, 128.5, 127.6, 127.6, 127.3, 127.3, 126.5, 38.1; HRMS (TOF ES+): m/z calcd for $\mathrm{C}_{17} \mathrm{H}_{14} \mathrm{NOS}_{2}\left[(\mathrm{M}+\mathrm{H})^{+}\right], 312.0511$, found, 312.0506 .

1-(3-Methyl-2-thioxo-2,3-dihydrothiazol-5-yl)-3-phenylprop-2-en-1-one (2u)<smiles>Cn1cc(C(=O)/C=C/c2ccccc2)sc1=S</smiles>

$\mathrm{V}_{\text {Petroleum ether }} / \mathrm{V}_{\text {Ethyl acetate }}=2: 1, \mathrm{R}_{f}=0.20$; Yellow solid: $88 \mathrm{mg}(68 \%) ; \mathrm{mp}=177-179{ }^{\circ} \mathrm{C} ;{ }^{1} \mathrm{H} \mathrm{NMR}$ $\left(600 \mathrm{MHz}, \mathrm{CDCl}_{3}\right): \delta=7.89$ (s, $\left.1 \mathrm{H}, \mathrm{C}=\mathrm{CHN}\right), 7.86(\mathrm{~d}, J=15.4 \mathrm{~Hz}, 1 \mathrm{H}, \mathrm{ArCH}=\mathrm{C}), 7.64$ (dd, $J=7.6$, $1.9 \mathrm{~Hz}, \mathrm{ArH}), 7.44-7.47$ (m, 3H, ArH), 7.07 (d, $J=15.5 \mathrm{~Hz}, 1 \mathrm{H}, \mathrm{C}=\mathrm{CHC}), 3.79$ (s, 3H, $\left.\mathrm{NCH}_{3}\right) ;{ }^{13} \mathrm{C}$ NMR (150 MHz, $\left.\mathrm{CDCl}_{3}\right): \delta=190.5,178.4,145.4,136.5,134.1,131.2,129.1,129.1,128.7,128.7$, 128.4, 119.7, 38.1; HRMS (TOF ES+): $\mathrm{m} / \mathrm{z}$ calcd for $\mathrm{C}_{13} \mathrm{H}_{12} \mathrm{NOS}_{2}\left[(\mathrm{M}+\mathrm{H})^{+}\right], 262.0355$, found, 262.0353 . 
(3-Methyl-2-thioxo-2,3-dihydrothiazol-5-yl)(thiophen-2-yl)methanone (2v)

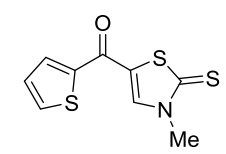

$\mathrm{V}_{\text {Petroleum ether }} / \mathrm{V}_{\text {Ethyl acetate }}=2: 1, \mathrm{R}_{f}=0.20$; Yellow solid: $87 \mathrm{mg}(72 \%) ; \mathrm{mp}=190-192{ }^{\circ} \mathrm{C} ;{ }^{1} \mathrm{H}$ NMR $\left(600 \mathrm{MHz}, \mathrm{CDCl}_{3}\right): \delta=7.88(\mathrm{~s}, 1 \mathrm{H}, \mathrm{C}=\mathrm{CHN}), 7.81(\mathrm{dd}, J=3.8,0.9 \mathrm{~Hz}, 1 \mathrm{H}, \mathrm{C}=\mathrm{CHC}), 7.74(\mathrm{dd}, J=$ $4.9,0.9 \mathrm{~Hz}, 1 \mathrm{H}, \mathrm{C}=\mathrm{CHC}), 7.21(\mathrm{dd}, J=4.9,3.9 \mathrm{~Hz}, 1 \mathrm{H}, \mathrm{SCH}=\mathrm{C}), 3.76\left(\mathrm{~s}, 3 \mathrm{H}, \mathrm{NCH}_{3}\right) ;{ }^{13} \mathrm{C} \mathrm{NMR}$ $\left(150 \mathrm{MHz}, \mathrm{CDCl}_{3}\right): \delta=190.0,174.7,141.5,137.8,134.4,132.7,128.4,125.4,38.1$; HRMS (TOF $\mathrm{ES}+): \mathrm{m} / \mathrm{z}$ calcd for $\mathrm{C}_{9} \mathrm{H}_{8} \mathrm{NOS}_{3}\left[(\mathrm{M}+\mathrm{H})^{+}\right], 241.9763$, found, 241.9757 .

Furan-2-yl(3-methyl-2-thioxo-2,3-dihydrothiazol-5-yl)methanone (2w)

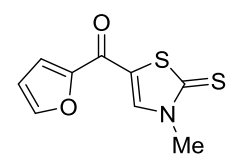

$\mathrm{V}_{\text {Petroleum ether }} / \mathrm{V}_{\text {Ethyl acetate }}=2: 1, \mathrm{R}_{f}=0.20$; Yellow solid: $83 \mathrm{mg}(74 \%) ; \mathrm{mp}=168-170{ }^{\circ} \mathrm{C} ;{ }^{1} \mathrm{H}$ NMR $\left(600 \mathrm{MHz}, \mathrm{CDCl}_{3}\right): \delta=8.27(\mathrm{~s}, 1 \mathrm{H}, \mathrm{OCH}=\mathrm{C}), 7.68(\mathrm{~s}, 1 \mathrm{H}, \mathrm{C}=\mathrm{CHN}), 7.41(\mathrm{~d}, J=3.4 \mathrm{~Hz}, 1 \mathrm{H}$, $\mathrm{C}=\mathrm{CHC}), 6.66(\mathrm{dd}, J=3.5,1.5145 .6, \mathrm{~Hz}, 1 \mathrm{H}, \mathrm{C}=\mathrm{CHC}), 3.80\left(\mathrm{~s}, 3 \mathrm{H}, \mathrm{NCH}_{3}\right) ;{ }^{13} \mathrm{C} \mathrm{NMR}(150 \mathrm{MHz}$, $\mathrm{CDCl}_{3}$ ): $\delta=190.4,169.3,151.9,133.8,125.1,119.1,113.1,38.1$; HRMS (TOF ES+): $\mathrm{m} / \mathrm{z}$ calcd for $\mathrm{C}_{9} \mathrm{H}_{8} \mathrm{NO}_{2} \mathrm{~S}_{2}\left[(\mathrm{M}+\mathrm{H})^{+}\right], 225.9991$, found, 225.9988.

(3-Methyl-2-thioxo-2,3-dihydrothiazol-5-yl)(pyridin-4-yl)methanone (2x)

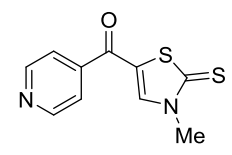

$\mathrm{V}_{\text {Petroleum ether }} / \mathrm{V}_{\text {Ethyl acetate }}=2: 1, \mathrm{R}_{f}=0.30$; Yellow solid: $76 \mathrm{mg}(64 \%) ; \mathrm{mp}=204-205{ }^{\circ} \mathrm{C} ;{ }^{1} \mathrm{H}$ NMR $\left(600 \mathrm{MHz}, \mathrm{CDCl}_{3}\right): \delta=8.87(\mathrm{~d}, J=5.9 \mathrm{~Hz}, 2 \mathrm{H}, \mathrm{N}=\mathrm{CHC}), 7.65(\mathrm{~s}, 1 \mathrm{H}, \mathrm{C}=\mathrm{CHN}), 7.59$ (d, $J=5.5 \mathrm{~Hz}$, $2 \mathrm{H}, \mathrm{C}=\mathrm{CHC}), 3.75\left(\mathrm{~s}, 3 \mathrm{H}, \mathrm{NCH}_{3}\right) ;{ }^{13} \mathrm{C}$ NMR $\left(150 \mathrm{MHz}, \mathrm{CDCl}_{3}\right): \delta=190.8,182.7,150.9,150.9$, 143.4, 140.0, 125.3, 121.6, 121.6, 38.2; HRMS (TOF ES+): $\mathrm{m} / \mathrm{z}$ calcd for $\mathrm{C}_{10} \mathrm{H}_{9} \mathrm{~N}_{2} \mathrm{OS}{ }_{2}\left[(\mathrm{M}+\mathrm{H})^{+}\right]$, 237.0151, found, 237.0148 .

(3-Methyl-2-thioxo-2,3-dihydrothiazol-5-yl)(pyrazin-2-yl)methanone (2y)

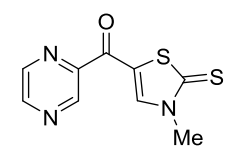

$\mathrm{V}_{\text {Petroleum ether }} / \mathrm{V}_{\text {Ethyl acetate }}=2: 1, \mathrm{R}_{f}=0.20$; Orange solid: $84 \mathrm{mg}(71 \%) ; \mathrm{mp}=188-190{ }^{\circ} \mathrm{C} ;{ }^{1} \mathrm{H}$ NMR $\left(600 \mathrm{MHz}, \mathrm{CDCl}_{3}\right): \delta=9.39(\mathrm{~m} .1 \mathrm{H}, \mathrm{CCH}=\mathrm{N}), 8.86(\mathrm{~d}, J=2.3 \mathrm{~Hz}, 1 \mathrm{H}, \mathrm{C}=\mathrm{CHN}), 8.70-8.71(\mathrm{~m}, 1 \mathrm{H}$, $\mathrm{NCH}=\mathrm{C}), 8.66$ (s, $1 \mathrm{H}, \mathrm{C}=\mathrm{CHN}), 3.81\left(\mathrm{~s}, 3 \mathrm{H}, \mathrm{NCH}_{3}\right) ;{ }^{13} \mathrm{C} \mathrm{NMR}\left(150 \mathrm{MHz}, \mathrm{CDCl}_{3}\right): \delta=191.9,178.7$, 148.1 147.0, 145.5, 142.8, 141.9, 123.1, 38.1; HRMS (TOF ES+): m/z calcd for $\mathrm{C}_{9} \mathrm{H}_{8} \mathrm{NO}_{2} \mathrm{~S}_{2}$ $\left[(\mathrm{M}+\mathrm{H})^{+}\right], 238.0103$, found, 238.0099 .

1-(3-Methyl-2-thioxo-2,3-dihydrothiazol-5-yl)ethan-1-one (2z)

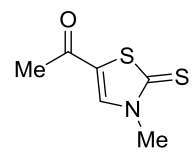


$\mathrm{V}_{\text {Petroleum ether }} / \mathrm{V}_{\text {Ethyl acetate }}=2: 1, \mathrm{R}_{f}=0.30$; Yellow solid: $62 \mathrm{mg}(72 \%) ; \mathrm{mp}=153-154{ }^{\circ} \mathrm{C} ;{ }^{1} \mathrm{H}$ NMR $\left(600 \mathrm{MHz}, \mathrm{CDCl}_{3}\right): \delta=7.62(\mathrm{~s}, 1 \mathrm{H}, \mathrm{C}=\mathrm{CHN}), 3.66\left(\mathrm{~s}, 3 \mathrm{H}, \mathrm{NCH}_{3}\right), 2.36\left(\mathrm{~s}, 3 \mathrm{H}, \mathrm{CCH}_{3}\right) ;{ }^{13} \mathrm{C} \mathrm{NMR}$ $\left(150 \mathrm{MHz}, \mathrm{CDCl}_{3}\right): \delta=190.7,186.7,137.0,127.3,38.0,26.4$; HRMS (TOF ES+): $\mathrm{m} / \mathrm{z}$ calcd for $\mathrm{C}_{6} \mathrm{H}_{8} \mathrm{NOS}_{2}\left[(\mathrm{M}+\mathrm{H})^{+}\right], 174.0042$, found, 174.0042 .

\section{Adamantan-1-yl(3-methyl-2-thioxo-2,3-dihydrothiazol-5-yl)methanone (2a')}

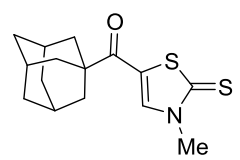

$\mathrm{V}_{\text {Petroleum ether }} / \mathrm{V}_{\text {Ethyl acetate }}=2: 1, \mathrm{R}_{f}=0.30$; Yellow solid: $138 \mathrm{mg}(95 \%) ; \mathrm{mp}=184-186{ }^{\circ} \mathrm{C} ;{ }^{1} \mathrm{H}$ NMR $\left(600 \mathrm{MHz}, \mathrm{CDCl}_{3}\right): \delta=7.82(\mathrm{~s}, 1 \mathrm{H}, \mathrm{C}=\mathrm{CHN}), 3.74\left(\mathrm{~s}, 3 \mathrm{H}, \mathrm{NCH}_{3}\right), 2.12-2.14\left(\mathrm{~m}, 3 \mathrm{H}, \mathrm{CCH}_{2} \mathrm{C}\right), 1.97$ (d, $J=2.3 \mathrm{~Hz}, 6 \mathrm{H}, \mathrm{CCH}_{2} \mathrm{C}$ ), 1.81 (d, $\left.J=12.4 \mathrm{~Hz}, 3 \mathrm{H}, \mathrm{CCHC}\right), 1.75$ (d, $J=11.7 \mathrm{~Hz}, 3 \mathrm{H}, \mathrm{CCH}_{2} \mathrm{C}$ ); ${ }^{13} \mathrm{C}$ NMR $\left(150 \mathrm{MHz}, \mathrm{CDCl}_{3}\right): \delta=195.2,189.4,137.4,123.9,46.6,39.0,39.0,39.0,37.9,36.4,36.4$, 36.4, 27.9, 27.9, 27.9; HRMS (TOF ES+): $\mathrm{m} / \mathrm{z}$ calcd for $\mathrm{C}_{15} \mathrm{H}_{20} \mathrm{NOS}_{2}\left[(\mathrm{M}+\mathrm{H})^{+}\right], 293.0908$, found, 293.0911

((3S,9S,10R,13S,14S,17S)-3-Hydroxy-10,13-dimethyl-2,3,4,7,8,9,10,11,12,13,14,15,16,17-tetrade cahydro-1H-cyclopenta[a]phenanthren-17-yl)(3-methyl-2-thioxo-2,3-dihydrothiazol-5-yl)metha none (2b')

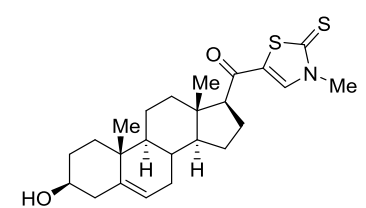

$\mathrm{V}_{\text {Petroleum ether }} / \mathrm{V}_{\text {Ethyl acetate }}=2: 1, \mathrm{R}_{f}=0.20$; Beige solid: $131 \mathrm{mg}(61 \%) ; \mathrm{mp}=185-187{ }^{\circ} \mathrm{C} ;{ }^{1} \mathrm{H}$ NMR $\left(600 \mathrm{MHz}, \mathrm{CDCl}_{3}\right): \delta=7.63(\mathrm{~s}, 1 \mathrm{H}, \mathrm{C}=\mathrm{CHN}), 5.35(\mathrm{~s}, 1 \mathrm{H}, \mathrm{C}=\mathrm{CH}), 3.71\left(\mathrm{~s}, 3 \mathrm{H}, \mathrm{NCH}_{3}\right), 3.51-3.53$ (m, 1H, OCHC), 2.84-2.87 (m, 1H, CH), 2.21-2.34 (m, 3H, CH), 2.01-2.04 (m, 1H, CH), 1.75-1.85 (m, 6H, CH), 1.61-1.62 (m, 2H, CH), 1.41-1.49 (m, 3H, CH), 1.31-1.37 (m, 2H, CH), 1.22-1.24 (m, $1 \mathrm{H}, \mathrm{CH}), 1.05-1.07(\mathrm{~m}, 1 \mathrm{H}, \mathrm{CH}), 0.99\left(\mathrm{~s}, 3 \mathrm{H}, \mathrm{CH}_{3}\right), 0.65\left(\mathrm{~s}, 3 \mathrm{H}, \mathrm{CH}_{3}\right) ;{ }^{13} \mathrm{C}$ NMR $(150 \mathrm{MHz}$, $\left.\mathrm{CDCl}_{3}\right): \delta=190.5,190.5,140.8,136.4,129.1,121.3,71.7,59.2,57.4,50.0,45.6,42.2,39.6,38.1$, 37.3, 36.6, 32.1, 31.9, 31.6, 24.8, 24.2, 21.2, 19.4, 13.7; HRMS (TOF ES $\left.{ }^{+}\right): \mathrm{m} / \mathrm{z}$ calcd for $\mathrm{C}_{24} \mathrm{H}_{34} \mathrm{NO}_{2} \mathrm{~S}_{2}\left[(\mathrm{M}+\mathrm{H})^{+}\right], 432.2025$, found, 432.2023 .

\section{(3,4-Dimethyl-2-thioxo-2,3-dihydrothiazol-5-yl)(phenyl)methanone (2c')}

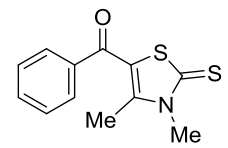

$\mathrm{V}_{\text {Petroleum ether }} / \mathrm{V}_{\text {Ethyl acetate }}=2: 1, \mathrm{R}_{f}=0.30$; Yellow solid: $76 \mathrm{mg}(61 \%) ; \mathrm{mp}=128-130{ }^{\circ} \mathrm{C} ;{ }^{1} \mathrm{H}$ NMR (600 MHz, DMSO- $\left.d_{6}\right): \delta=7.75-7.77(\mathrm{~m}, 2 \mathrm{H}, \mathrm{ArH}), 7.67-7.70(\mathrm{~m}, 1 \mathrm{H}, \mathrm{ArH}), 7.55-7.57(\mathrm{~m}, 2 \mathrm{H}$, $\mathrm{ArH}), 3.67$ (s, $3 \mathrm{H}, \mathrm{NCH}_{3}$ ), 2.37 (s, 3H, $\left.\mathrm{CCH}_{3}\right) ;{ }^{13} \mathrm{C} \mathrm{NMR}\left(150 \mathrm{MHz}, \mathrm{DMSO}-d_{6}\right): \delta=187.8,186.5$, 149.5, 138.7, 133.5, 129.3 129.3, 129.0, 129.0, 119.6, 35.1, 16.3; HRMS (TOF ES $\left.{ }^{+}\right): \mathrm{m} / \mathrm{z}$ calcd for $\mathrm{C}_{12} \mathrm{H}_{12} \mathrm{NOS}_{2}\left[(\mathrm{M}+\mathrm{H})^{+}\right], 250.0355$, found, 250.0364 .

(3-Methyl-4-phenyl-2-thioxo-2,3-dihydrothiazol-5-yl)(phenyl)methanone (2d') 


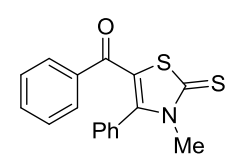

$\mathrm{V}_{\text {Petroleum ether }} / \mathrm{V}_{\text {Ethyl acetate }}=2: 1, \mathrm{R}_{f}=0.30$; Yellow solid: $115 \mathrm{mg}(74 \%) ; \mathrm{mp}=151-152{ }^{\circ} \mathrm{C} ;{ }^{1} \mathrm{H}$ NMR $\left(600 \mathrm{MHz}, \mathrm{DMSO}-d_{6}\right): \delta=7.38-7.43$ (m, 4H, ArH), 7.30-7.34 (m, 2H, ArH), 7.24-7.27 (m, 2H, $\operatorname{ArH}), 7.15(\mathrm{t}, J=7.6 \mathrm{~Hz}, 2 \mathrm{H}, \mathrm{ArH}), 3.42\left(\mathrm{~s}, 3 \mathrm{H}, \mathrm{NCH}_{3}\right) ;{ }^{13} \mathrm{C}$ NMR $\left(150 \mathrm{MHz}, \mathrm{DMSO}-d_{6}\right): \delta=188.2$, 186.9, 149.6, 137.2, 132.6, 131.0, 131.0, 130.6, 129.7, 129.0, 129.0, 128.8, 128.8, 128.3, 128.3, 123.3, 37.0; HRMS (TOF ES ${ }^{+}$): m/z calcd for $\mathrm{C}_{17} \mathrm{H}_{14} \mathrm{NOS}_{2}\left[(\mathrm{M}+\mathrm{H})^{+}\right], 312.0511$, found, 312.0519 .

\section{3-Methyl-2-thioxo-1,2,3,5,6,7-hexahydro-4H-indol-4-one (2e')}

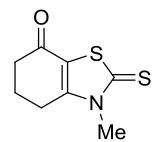

$\mathrm{V}_{\text {Petroleum ether }} / \mathrm{V}_{\text {Ethyl acetate }}=1: 1, \mathrm{R}_{f}=0.20$; Beige solid: $69 \mathrm{mg}(69 \%) ; \mathrm{mp}=170-172{ }^{\circ} \mathrm{C} ;{ }^{1} \mathrm{H}$ NMR $\left(600 \mathrm{MHz}, \mathrm{CDCl}_{3}\right): \delta=3.60\left(\mathrm{~s}, 3 \mathrm{H}, \mathrm{NCH}_{3}\right), 2.76-2.78\left(\mathrm{~m}, 2 \mathrm{H}, \mathrm{CCH}_{2} \mathrm{C}\right), 2.53-2.55\left(\mathrm{~m}, 2 \mathrm{H}, \mathrm{CCH}_{2} \mathrm{C}\right)$, 2.19-2.24 (m, $\left.2 \mathrm{H}, \mathrm{CCH}_{2} \mathrm{C}\right) ;{ }^{13} \mathrm{C} \mathrm{NMR}\left(150 \mathrm{MHz}, \mathrm{CDCl}_{3}\right): \delta=191.2,188.4,154.3,120.5,37.1,34.4$, 25.0, 22.0; HRMS (TOF ES+): m/z calcd for $\mathrm{C}_{8} \mathrm{H}_{10} \mathrm{NOS}_{2}\left[(\mathrm{M}+\mathrm{H})^{+}\right]$, 200.0198, found, 200.0202.

3,5,5-Trimethyl-2-thioxo-2,3,5,6-tetrahydrobenzo[d]thiazol-7(4H)-one (2f')

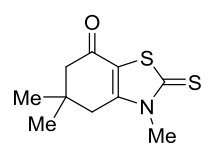

$\mathrm{V}_{\text {Petroleum ether }} / \mathrm{V}_{\text {Ethyl acetate }}=1: 1, \mathrm{R}_{f}=0.30$; Beige solid: $76 \mathrm{mg}(67 \%) ; \mathrm{mp}=153-154{ }^{\circ} \mathrm{C} ;{ }^{1} \mathrm{H}$ NMR $\left(600 \mathrm{MHz}, \mathrm{CDCl}_{3}\right): \delta=3.59\left(\mathrm{~s}, 3 \mathrm{H}, \mathrm{NCH}_{3}\right), 2.61\left(\mathrm{~s}, 2 \mathrm{H}, \mathrm{CCH}_{2} \mathrm{C}\right), 2.41\left(\mathrm{~s}, 2 \mathrm{H}, \mathrm{CCH}_{2} \mathrm{C}\right), 1.14(\mathrm{~s}, 6 \mathrm{H}$, $\left.\mathrm{CCH}_{3}\right) ;{ }^{13} \mathrm{C}$ NMR $\left(150 \mathrm{MHz}, \mathrm{CDCl}_{3}\right): \delta=191.5,188.1,152.8,119.3,51.1,38.8,34.7,34.4,28.6$, 28.6; HRMS (TOF ES+): $\mathrm{m} / \mathrm{z}$ calcd for $\mathrm{C}_{10} \mathrm{H}_{14} \mathrm{NOS}_{2}\left[(\mathrm{M}+\mathrm{H})^{+}\right]$, 228.0511, found, 228.0510 .

Phenyl(3-phenyl-2-thioxo-2,3-dihydrothiazol-5-yl)methanone (4a)

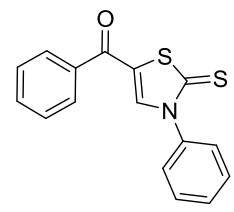

$\mathrm{V}_{\text {Petroleum ether }} / \mathrm{V}_{\text {Ethyl acetate }}=3: 1, \mathrm{R}_{f}=0.20$; Yellow solid: $111 \mathrm{mg}(75 \%) ; \mathrm{mp}=108-110{ }^{\circ} \mathrm{C} ;{ }^{1} \mathrm{H}$ NMR (600 MHz, CDCl3): $\delta=7.75-7.71(\mathrm{~m}, 2 \mathrm{H}, \mathrm{ArH}), 7.61(\mathrm{~s}, 1 \mathrm{H}, \mathrm{C}=\mathrm{CHN}), 7.55(\mathrm{t}, J=7.5 \mathrm{~Hz}, 1 \mathrm{H}$, $\mathrm{ArH}), 7.49-7.39(\mathrm{~m}, 7 \mathrm{H}, \mathrm{ArH}) ;{ }^{13} \mathrm{C} \mathrm{NMR}\left(150 \mathrm{MHz}, \mathrm{CDCl}_{3}\right): \delta=191.1,184.3,139.1,137.9,136.7$, 133.1 130.0, 129.8, 129.8, 129.0, 129.0, 128.5, 128.5, 127.0, 126.7, 126.7; HRMS (TOF ES+): m/z calcd for $\mathrm{C}_{16} \mathrm{H}_{12} \mathrm{NOS}_{2}\left[(\mathrm{M}+\mathrm{H})^{+}\right]$, 298.0355, found, 298.0356 .

(3-(4-Methoxyphenyl)-2-thioxo-2,3-dihydrothiazol-5-yl)(phenyl)methanone (4b)

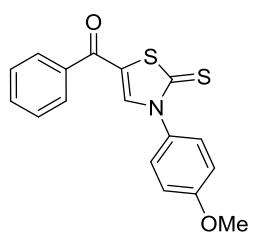


$\mathrm{V}_{\text {Petroleum ether }} / \mathrm{V}_{\text {Ethyl acetate }}=4: 1, \mathrm{R}_{f}=0.20$; Yellow solid: $142 \mathrm{mg}(87 \%) ; \mathrm{mp}=152-154{ }^{\circ} \mathrm{C} ;{ }^{1} \mathrm{H}$ NMR (600 MHz, $\left.\mathrm{CDCl}_{3}\right): \delta=7.81-7.82(\mathrm{~m}, 2 \mathrm{H}, \mathrm{ArH}), 7.69(\mathrm{~s}, 1 \mathrm{H}, \mathrm{C}=\mathrm{CHN}), 7.64(\mathrm{t}, J=7.5 \mathrm{~Hz}, 2 \mathrm{H}$, $\operatorname{ArH}), 7.53(\mathrm{t}, J=7.7 \mathrm{~Hz}, 2 \mathrm{H}, \mathrm{ArH}), 7.41(\mathrm{~d}, J=8.9 \mathrm{~Hz}, 2 \mathrm{H}, \mathrm{ArH}), 7.05(\mathrm{~d}, J=8.9 \mathrm{~Hz}, 2 \mathrm{H}, \operatorname{ArH})$, $3.88\left(\mathrm{~s}, 3 \mathrm{H}, \mathrm{OCH}_{3}\right) ;{ }^{13} \mathrm{C} \mathrm{NMR}\left(150 \mathrm{MHz}, \mathrm{CDCl}_{3}\right): \delta=191.4,184.3,160.4,139.5,136.7,133.1$, 130.6, 129.0, 129.0, 128.5, 128.5, 127.9, 127.9, 126.7, 114.8, 114.8, 55.7; HRMS (TOF ES+): m/z calcd for $\mathrm{C}_{17} \mathrm{H}_{14} \mathrm{NO}_{2} \mathrm{~S}_{2}\left[(\mathrm{M}+\mathrm{H})^{+}\right], 328.0460$, found, 328.0464.

(3-Ethyl-2-thioxo-2,3-dihydrothiazol-5-yl)(phenyl)methanone (4c)

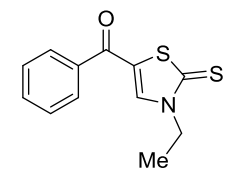

$\mathrm{V}_{\text {Petroleum ether }} / \mathrm{V}_{\text {Ethyl acetate }}=3: 1, \mathrm{R}_{f}=0.30$; Yellow solid: $90 \mathrm{mg}(72 \%) ; \mathrm{mp}=129-131{ }^{\circ} \mathrm{C} ;{ }^{1} \mathrm{H}$ NMR $\left(600 \mathrm{MHz}, \mathrm{CDCl}_{3}\right): \delta=7.79(\mathrm{~s}, 1 \mathrm{H}, \mathrm{C}=\mathrm{CHN}), 7.77-7.78(\mathrm{~m}, 1 \mathrm{H}, \mathrm{ArH}), 7.66(\mathrm{~d}, J=5.6 \mathrm{~Hz}, 2 \mathrm{H}$, $\operatorname{ArH}), 7.55$ (t, $J=7.7 \mathrm{~Hz}, 2 \mathrm{H}, \mathrm{ArH}), 4.28$ (q, $J=7.3 \mathrm{~Hz}, 2 \mathrm{H}, \mathrm{NCH}_{2} \mathrm{C}$ ), 1.46 (t, $J=7.3 \mathrm{~Hz}, 3 \mathrm{H}$, $\left.\mathrm{CCH}_{3}\right) ;{ }^{13} \mathrm{C}$ NMR $\left(150 \mathrm{MHz}, \mathrm{CDCl}_{3}\right): \delta=190.1,184.2,137.8,136.9,133.1,129.0,129.0,128.5$, 128.5, 126.8, 45.6, 14.0; HRMS (TOF ES+): $\mathrm{m} / \mathrm{z}$ calcd for $\mathrm{C}_{12} \mathrm{H}_{12} \mathrm{NOS}_{2}\left[(\mathrm{M}+\mathrm{H})^{+}\right], 250.0355$, found, 250.0359 .

(3-Ethyl-2-thioxo-2,3-dihydrothiazol-5-yl)(p-tolyl)methanone (4d)

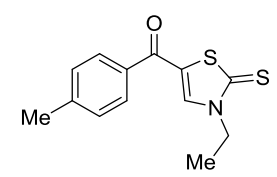

$\mathrm{V}_{\text {Petroleum ether }} / \mathrm{V}_{\text {Ethyl acetate }}=3: 1, \mathrm{R}_{f}=0.20$; Yellow solid: $95 \mathrm{mg}(72 \%) ; \mathrm{mp}=138-140{ }^{\circ} \mathrm{C} ;{ }^{1} \mathrm{H}$ NMR $\left(600 \mathrm{MHz}, \mathrm{CDCl}_{3}\right): \delta=7.70-7.71(\mathrm{~d}, J=7.7 \mathrm{~Hz}, 2 \mathrm{H}, \mathrm{ArH}), 7.65(\mathrm{~s}, 1 \mathrm{H}, \mathrm{C}=\mathrm{CHN}), 7.34(\mathrm{~d}, J=7.9$ $\mathrm{Hz}, 2 \mathrm{H}, \mathrm{ArH}), 4.27$ (q, J=7.3 Hz, 2H, $\left.\mathrm{NCCH}_{2}\right), 2.47$ (s, 3H, $\left.\mathrm{ArCH}_{3}\right), 1.46$ (t, $J=7.3 \mathrm{~Hz}, 3 \mathrm{H}, \mathrm{CCH}_{3}$ ); ${ }^{13} \mathrm{C}$ NMR (150 MHz, $\left.\mathrm{CDCl}_{3}\right): \delta=189.9,183.9,144.1,137.4,134.2,129.7,129.7,128.7,128.7$, 126.9, 45.6, 21.8, 14.0; HRMS (TOF ES+): $\mathrm{m} / \mathrm{z}$ calcd for $\mathrm{C}_{13} \mathrm{H}_{14} \mathrm{NOS}_{2}\left[(\mathrm{M}+\mathrm{H})^{+}\right], 264.0511$, found, 264.0514.

(2,4-Dichlorophenyl)(3-ethyl-2-thioxo-2,3-dihydrothiazol-5-yl)methanone (4e)<smiles></smiles>

$\mathrm{V}_{\text {Petroleum ether }} / \mathrm{V}_{\text {Ethyl acetate }}=4: 1, \mathrm{R}_{f}=0.20$; Yellow solid: $111 \mathrm{mg}(70 \%) ; \mathrm{mp}=127-129{ }^{\circ} \mathrm{C} ;{ }^{1} \mathrm{H}$ NMR $\left(600 \mathrm{MHz}, \mathrm{CDCl}_{3}\right): \delta=7.54(\mathrm{~d}, J=1.6 \mathrm{~Hz}, 1 \mathrm{H}, \mathrm{C}=\mathrm{CHN}), 7.39-7.42(\mathrm{~m}, 3 \mathrm{H}, \mathrm{ArH}), 4.22(\mathrm{q}, J=7.3$ $\left.\mathrm{Hz}, 2 \mathrm{H}, \mathrm{NCH}_{2} \mathrm{C}\right), 1.43\left(\mathrm{t}, J=7.3 \mathrm{~Hz}, 3 \mathrm{H}, \mathrm{CCH}_{3}\right) ;{ }^{13} \mathrm{C} \mathrm{NMR}\left(150 \mathrm{MHz}, \mathrm{CDCl}_{3}\right): \delta=190.5,182.0$, 139.1, 137.7, 135.1 132.3, 130.6, 129.8, 127.5, 126.4, 15.6, 13.9; HRMS (TOF ES+): $\mathrm{m} / \mathrm{z}$ calcd for $\mathrm{C}_{12} \mathrm{H}_{10} \mathrm{Cl}_{2} \mathrm{NOS}_{2}[(\mathrm{M}+\mathrm{H})+], 317.9575$, found, 317.9571 .

(3-Cyclohexyl-2-thioxo-2,3-dihydrothiazol-5-yl)(phenyl)methanone (4f) 


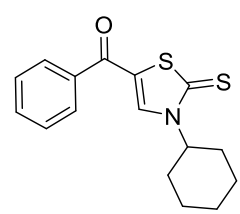

$\mathrm{V}_{\text {Petroleum ether }} / \mathrm{V}_{\text {Ethyl acetate }}=4: 1, \mathrm{R}_{f}=0.20$; Yellow solid: $136 \mathrm{mg}(90 \%) ; \mathrm{mp}=116-118{ }^{\circ} \mathrm{C} ;{ }^{1} \mathrm{H}$ NMR $\left(600 \mathrm{MHz}, \mathrm{CDCl}_{3}\right): \delta=7.79(\mathrm{~d}, J=7.6 \mathrm{~Hz}, 2 \mathrm{H}, \mathrm{ArH}), 7.67(\mathrm{~s}, 1 \mathrm{H}, \mathrm{C}=\mathrm{CHN}), 7.65-7.66(\mathrm{~m}, 1 \mathrm{H}$, ArH), 7.55-7.57 (m, 2H, ArH), 4.98-5.03 (m, 1H, NCHC), 2.14 (d, $\left.J=11.1 \mathrm{~Hz}, 2 \mathrm{H}, \mathrm{CCH}_{2} \mathrm{C}\right), 1.94$ (d, $\left.J=13.8 \mathrm{~Hz}, 2 \mathrm{H}, \mathrm{CCH}_{2} \mathrm{C}\right), 1.80\left(\mathrm{~d}, J=13.3 \mathrm{~Hz}, 1 \mathrm{H}, \mathrm{CCH}_{2} \mathrm{C}\right), 1.50-1.57\left(\mathrm{~m}, 2 \mathrm{H}, \mathrm{CCH}_{2} \mathrm{C}\right)$, 1.39-1.42 (m, $\left.2 \mathrm{H}, \mathrm{CCH}_{2} \mathrm{C}\right), 1.19-1.27\left(\mathrm{~m}, 1 \mathrm{H}, \mathrm{CCH}_{2} \mathrm{C}\right) ;{ }^{13} \mathrm{C}$ NMR $\left(150 \mathrm{MHz}, \mathrm{CDCl}_{3}\right): \delta=189.9$, 184.3, 137.0, 135.3, 133.1, 129.0, 129.0, 128.5, 128.5, 126.8, 58.4, 32.6, 32.6, 25.5, 25.5, 25.1; HRMS (TOF ES+): $\mathrm{m} / \mathrm{z}$ calcd for $\mathrm{C}_{16} \mathrm{H}_{18} \mathrm{NOS}_{2}\left[(\mathrm{M}+\mathrm{H})^{+}\right], 304.4455$, found, 304.4460.

(3-Benzyl-2-thioxo-2,3-dihydrothiazol-5-yl)(phenyl)methanone (4g)

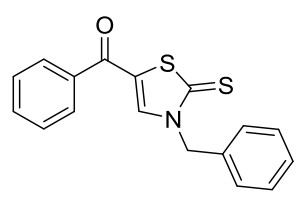

$\mathrm{V}_{\text {Petroleum ether }} / \mathrm{V}_{\text {Ethyl acetate }}=5: 1, \mathrm{R}_{f}=0.20$; Yellow solid: $114 \mathrm{mg}(73 \%) ; \mathrm{mp}=104-106{ }^{\circ} \mathrm{C} ;{ }^{1} \mathrm{H}$ NMR (600 MHz, $\left.\mathrm{CDCl}_{3}\right): \delta=7.61$ (dd, $\left.J=8.2,1.2 \mathrm{~Hz}, 2 \mathrm{H}, \mathrm{ArH}\right), 7.50-7.54(\mathrm{~m}, 1 \mathrm{H}, \mathrm{ArH}), 7.43(\mathrm{~s}, 1 \mathrm{H}$, $\mathrm{C}=\mathrm{CHN}$ ), $7.39(\mathrm{t}, J=7.8 \mathrm{~Hz}, 2 \mathrm{H}, \mathrm{ArH}), 7.31$ (dddd, $J=11.5,6.8,4.5,2.4 \mathrm{~Hz}, 3 \mathrm{H}, \mathrm{ArH}), 7.23-7.27$ $(\mathrm{m}, 2 \mathrm{H}, \mathrm{ArH}), 5.32\left(\mathrm{~s}, 2 \mathrm{H}, \mathrm{CCH}_{2} \mathrm{C}\right) ;{ }^{13} \mathrm{C}$ NMR $\left(150 \mathrm{MHz}, \mathrm{CDCl}_{3}\right): \delta=190.7,184.1,137.8,135.7$, 134.4, 133.1, 129.3, 129.3, 128.9, 128.9, 128.9, 128.5, 128.5, 128.2, 128.2, 126.7, 53.2; HRMS (TOF ES+): $\mathrm{m} / \mathrm{z}$ calcd for $\mathrm{C}_{17} \mathrm{H}_{14} \mathrm{NOS}_{2}\left[(\mathrm{M}+\mathrm{H})^{+}\right], 312.0511$, found, 312.0512 .

(3-Phenethyl-2-thioxo-2,3-dihydrothiazol-5-yl)(phenyl)methanone (4h)

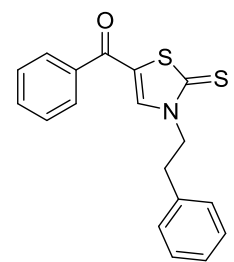

$\mathrm{V}_{\text {Petroleum ether }} / \mathrm{V}_{\text {Ethyl acetate }}=4: 1, \mathrm{R}_{f}=0.20$; Yellow solid: $143 \mathrm{mg}(88 \%) ; \mathrm{mp}=108-109{ }^{\circ} \mathrm{C} ;{ }^{1} \mathrm{H}$ NMR $\left(600 \mathrm{MHz}, \mathrm{CDCl}_{3}\right): \delta=7.58(\mathrm{t}, J=7.4 \mathrm{~Hz}, 1 \mathrm{HArH}), 7.44-7.36(\mathrm{~m}, 5 \mathrm{H}, \mathrm{ArH}), 7.34$ (d, $J=7.1 \mathrm{~Hz}$, 2H, ArH), 7.24-7.19 (m, 2H, ArH), $6.91(\mathrm{~s}, 1 \mathrm{H}, \mathrm{C}=\mathrm{CHN}), 4.44\left(\mathrm{t}, J=6.5 \mathrm{~Hz}, 2 \mathrm{H}, \mathrm{NCH}_{2} \mathrm{C}\right), 3.21(\mathrm{t}$, $\left.J=6.5 \mathrm{~Hz}, 2 \mathrm{H}, \mathrm{CCH}_{2} \mathrm{C}\right) ;{ }^{13} \mathrm{C} \mathrm{NMR}\left(150 \mathrm{MHz}, \mathrm{CDCl}_{3}\right): \delta=190.1,184.2,139.2,137.3,136.5,132.8$, 129.3, 129.3, 129.2 129.2, 128.8, 128.8, 128.5, 128.5, 127.2, 125.7, 52.2, 33.5; HRMS (TOF ES+): $\mathrm{m} / \mathrm{z}$ calcd for $\mathrm{C}_{18} \mathrm{H}_{16} \mathrm{NOS}_{2}\left[(\mathrm{M}+\mathrm{H})^{+}\right], 326.0668$, found, 326.0670 .

Phenyl(3-(2-(tetrahydrofuran-2-yl)ethyl)-2-thioxo-2,3-dihydrothiazol-5-yl)methanone (4i)<smiles>O=C(c1ccccc1)c1cn(CCC2CCCO2)c(=S)s1</smiles> 
$\mathrm{V}_{\text {Petroleum ether }} / \mathrm{V}_{\text {Ethyl acetate }}=3: 1, \mathrm{R}_{f}=0.30 ;$ Yellow solid: $142 \mathrm{mg}(89 \%) ; \mathrm{mp}=141-143{ }^{\circ} \mathrm{C} ;{ }^{1} \mathrm{H}$ NMR $\left(600 \mathrm{MHz}, \mathrm{CDCl}_{3}\right): \delta=7.86(\mathrm{~s}, 1 \mathrm{H}, \mathrm{C}=\mathrm{CHN}), 7.84-7.76(\mathrm{~m}, 2 \mathrm{H}, \mathrm{ArH}), 7.65(\mathrm{t}, J=7.5 \mathrm{~Hz}, 1 \mathrm{H}$, $\mathrm{ArH}), 7.54(\mathrm{t}, J=7.7 \mathrm{~Hz}, 2 \mathrm{H}, \mathrm{ArH}), 4.62-4.64\left(\mathrm{~m}, 1 \mathrm{H}, \mathrm{OCH}_{2} \mathrm{C}\right), 4.24-4.27\left(\mathrm{~m}, 1 \mathrm{H}, \mathrm{OCH}_{2} \mathrm{C}\right)$, 3.85-3.89 (m, $\left.1 \mathrm{H}, \mathrm{NCH}_{2} \mathrm{C}\right), 3.78-3.81\left(\mathrm{~m}, 1 \mathrm{H}, \mathrm{CCH}_{2} \mathrm{C}\right), 2.11-2.17\left(\mathrm{~m}, 1 \mathrm{H}, \mathrm{CCH}_{2} \mathrm{C}\right), 1.92-1.96(\mathrm{~m}$, $\left.2 \mathrm{H}, \mathrm{CCH}_{2} \mathrm{C}\right), 1.62-1.68\left(\mathrm{~m}, 2 \mathrm{H}, \mathrm{CCH}_{2} \mathrm{C}\right) ;{ }^{13} \mathrm{C}$ NMR $\left(150 \mathrm{MHz}, \mathrm{CDCl}_{3}\right): \delta=191.0,184.5,140.0$, 136.9, 133.0, 128.9, 128.9, 128.7, 128.7, 125.9, 76.5, 68.4, 53.0, 28.8, 25.8; HRMS (TOF ES+): m/z calcd for $\mathrm{C}_{16} \mathrm{H}_{18} \mathrm{NO}_{2} \mathrm{~S}_{2}\left[(\mathrm{M}+\mathrm{H})^{+}\right], 320.0773$, found, 320.0778 .

(4-Methoxyphenyl)(3-(2-(tetrahydrofuran-2-yl)ethyl)-2-thioxo-2,3-dihydrothiazol-5-yl)methan one $(4 \mathbf{j})$

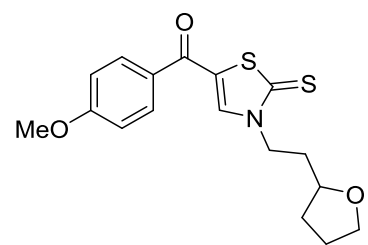

$\mathrm{V}_{\text {Petroleum ether }} / \mathrm{V}_{\text {Ethyl acetate }}=4: 1, \mathrm{R}_{f}=0.30 ;$ Yellow solid: $148 \mathrm{mg}(85 \%) ; \mathrm{mp}=83-85{ }^{\circ} \mathrm{C} ;{ }^{1} \mathrm{H}$ NMR $\left(600 \mathrm{MHz}, \mathrm{CDCl}_{3}\right): \delta=7.77(\mathrm{~s}, 1 \mathrm{H}, \mathrm{C}=\mathrm{CHN}), 7.74(\mathrm{~d}, J=8.8 \mathrm{~Hz}, 2 \mathrm{H}, \mathrm{ArH}), 6.93(\mathrm{~d}, J=8.8 \mathrm{~Hz}, 2 \mathrm{H}$, $\mathrm{ArH}), 4.54(\mathrm{dd}, J=13.9,2.4 \mathrm{~Hz}, 1 \mathrm{H}, \mathrm{OCHC}), 4.16-4.20\left(\mathrm{~m}, 1 \mathrm{H}, \mathrm{OCH}_{2} \mathrm{C}\right), 3.97-4.00(\mathrm{~m}, 1 \mathrm{H}$, $\left.\mathrm{OCH}_{2} \mathrm{C}\right), 3.83\left(\mathrm{~s}, 3 \mathrm{H}, \mathrm{ArOCH}_{3}\right), 3.78-3.81\left(\mathrm{~m}, 1 \mathrm{H}, \mathrm{NCH}_{2} \mathrm{C}\right), 3.70-3.73(\mathrm{~m}, 1 \mathrm{H}), 2.03-2.08(\mathrm{~m}, 1 \mathrm{H}$, $\left.\mathrm{CCH}_{2} \mathrm{C}\right), 1.84-1.87\left(\mathrm{~m}, 2 \mathrm{H}, \mathrm{CCH}_{2} \mathrm{C}\right), 1.54-1.59\left(\mathrm{~m}, 1 \mathrm{H}, \mathrm{CCH}_{2} \mathrm{C}\right) ;{ }^{13} \mathrm{C} \mathrm{NMR}\left(150 \mathrm{MHz}, \mathrm{CDCl}_{3}\right): \delta=$ 190.7, 183.0, 163.6, 139.1, 131.1, 131.1, 129.4, 126.2, 114.16, 114.16, 76.5, 68.4, 55.6, 53.0, 28.8, 25.8; HRMS (TOF ES+): $\mathrm{m} / \mathrm{z}$ calcd for $\mathrm{C}_{17} \mathrm{H}_{20} \mathrm{NO}_{3} \mathrm{~S}_{2}\left[(\mathrm{M}+\mathrm{H})^{+}\right], 350.0879$, found, 350.0872 .

(3-(3-(2-(1-methyl-1H-indol-3-yl)ethyl)-2-thioxo-2,3-dihydrothiazol-5-yl)(p-tolyl)methanone $(4 k)$

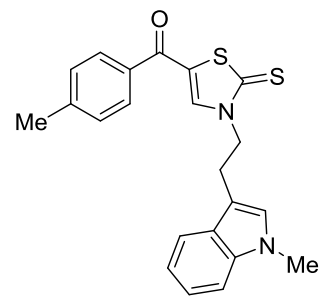

$\mathrm{V}_{\text {Petroleum ether }} / \mathrm{V}_{\text {Ethyl acetate }}=4: 1, \mathrm{R}_{f}=0.20$; Brown oil: $166 \mathrm{mg},(85 \%) ;{ }^{1} \mathrm{H}$ NMR $\left(600 \mathrm{MHz}, \mathrm{CDCl}_{3}\right): \delta$ $=7.45(\mathrm{~d}, J=7.9 \mathrm{~Hz}, 1 \mathrm{H}, \operatorname{ArH}), 7.33(\mathrm{~d}, J=8.2 \mathrm{~Hz}, 1 \mathrm{H}, \operatorname{ArH}), 7.23(\mathrm{t}, J=7.7 \mathrm{~Hz}, 1 \mathrm{H}, \operatorname{ArH}), 7.08(\mathrm{t}$, $J=7.4 \mathrm{~Hz}, 1 \mathrm{H}, \mathrm{ArH}), 6.96(\mathrm{~d}, J=7.9 \mathrm{~Hz}, 2 \mathrm{H}, \mathrm{ArH}), 6.82(\mathrm{~s}, 1 \mathrm{H}, \mathrm{C}=\mathrm{CHN}), 6.81(\mathrm{~d}, J=4.4 \mathrm{~Hz}, 2 \mathrm{H}$, $\mathrm{ArH}), 6.68(\mathrm{~s}, 1 \mathrm{H}, \mathrm{C}=\mathrm{CHN}), 4.39\left(\mathrm{t}, J=6.3 \mathrm{~Hz}, 2 \mathrm{H}, \mathrm{CCH}_{2} \mathrm{C}\right), 3.70\left(\mathrm{~s}, 3 \mathrm{H}, \mathrm{NCH}_{3}\right), 3.27(\mathrm{t}, J=6.2$

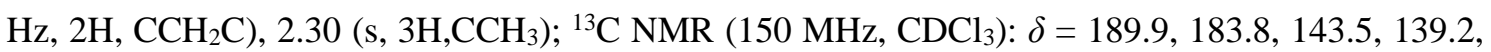
137.2, 133.8, 129.3, 129.3, 128.3, 128.3, 127.9, 127.4, 125.7, 122.3, 119.8, 118.4, 109.7, 109.6, 51.4, 32.8, 23.2, 21.7; HRMS (TOF ES+): $\mathrm{m} / \mathrm{z}$ calcd for $\mathrm{C}_{22} \mathrm{H}_{21} \mathrm{~N}_{2} \mathrm{OS}_{2}\left[(\mathrm{M}+\mathrm{H})^{+}\right], 393.1090$, found, 393.1104 .

3-Phenyl-2-thioxo-2,3,5,6-tetrahydrobenzo[d]thiazol-7(4H)-one (4l) 


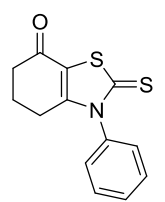

$\mathrm{V}_{\text {Petroleum ether }} / \mathrm{V}_{\text {Ethyl acetate }}=3: 1, \mathrm{R}_{f}=0.30$; Beige solid: $94 \mathrm{mg}(72 \%) ; \mathrm{mp}=217-219{ }^{\circ} \mathrm{C} ;{ }^{1} \mathrm{H}$ NMR $\left(600 \mathrm{MHz}, \mathrm{CDCl}_{3}\right): \delta=7.57-7.63(\mathrm{~m}, 3 \mathrm{H}, \mathrm{ArH}), 7.31-7.31(\mathrm{~m}, 2 \mathrm{H}, \mathrm{ArH}), 2.62-2.64(\mathrm{~m}, 2 \mathrm{H}$, $\left.\mathrm{CCH}_{2} \mathrm{C}\right), 2.47-2.49\left(\mathrm{~m}, 2 \mathrm{H}, \mathrm{CCH}_{2} \mathrm{C}\right), 2.17-2.21\left(\mathrm{~m}, 2 \mathrm{H}, \mathrm{CCH}_{2} \mathrm{C}\right) ;{ }^{13} \mathrm{C} \mathrm{NMR}\left(150 \mathrm{MHz}, \mathrm{CDCl}_{3}\right): \delta=$ 192.4 188.6, 154.9, 136.5, 130.3, 127.9, 120.8, 37.3, 25.7, 22.2; HRMS (TOF ES+): m/z calcd for $\mathrm{C}_{13} \mathrm{H}_{12} \mathrm{NOS}_{2}\left[(\mathrm{M}+\mathrm{H})^{+}\right], 262.0355$, found, 262.0352 .

\section{5,5-Dimethyl-3-phenyl-2-thioxo-2,3,5,6-tetrahydrobenzo[d]thiazol-7 $(4 \mathrm{H})$-one (4m)}

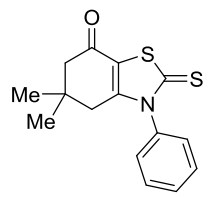

$\mathrm{V}_{\text {Petroleum ether }} / \mathrm{V}_{\text {Ethyl acetate }}=3: 1, \mathrm{R}_{f}=0.30 ;$ Beige solid: $114 \mathrm{mg}(73 \%) ; \mathrm{mp}=174-176{ }^{\circ} \mathrm{C} ;{ }^{1} \mathrm{H}$ NMR $\left(600 \mathrm{MHz}, \mathrm{CDCl}_{3}\right): \delta=7.58-7.64(\mathrm{~m}, 3 \mathrm{H}, \mathrm{ArH}), 7.28-7.29(\mathrm{~m}, 2 \mathrm{H}, \mathrm{ArH}), 2.50\left(\mathrm{~s}, 2 \mathrm{H}, \mathrm{CCH}_{2} \mathrm{C}\right)$, 2.33 (s, $2 \mathrm{H}, \mathrm{CCH}_{2} \mathrm{C}$ ), 1.13 (s, 6H, $\left.\mathrm{CCH}_{3}\right) ;{ }^{13} \mathrm{C} \mathrm{NMR}\left(150 \mathrm{MHz}, \mathrm{CDCl}_{3}\right): \delta=192.6,188.3,154.9$, 136.4 130.3, 130.3 127.9, 127.9, 119.6, 51.3, 39.4, 34.9, 28.3, 28.3; HRMS (TOF ES+): $\mathrm{m} / \mathrm{z}$ calcd for $\mathrm{C}_{15} \mathrm{H}_{15} \mathrm{NOS}_{2} \mathrm{Na}\left[(\mathrm{M}+\mathrm{Na})^{+}\right]$, 312.0487, found, 312.0483 .

\section{3-(2,6-Diisopropylphenyl)-2-thioxo-2,3,5,6-tetrahydrobenzo[d]thiazol-7(4H)-one (4n)}

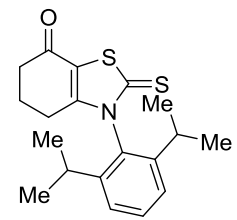

$\mathrm{V}_{\text {Petroleum ether }} / \mathrm{V}_{\text {Ethyl acetate }}=4: 1, \mathrm{R}_{f}=0.20$; Beige solid: $124 \mathrm{mg}(72 \%) ; \mathrm{mp}=220-222{ }^{\circ} \mathrm{C} ;{ }^{1} \mathrm{H}$ NMR $\left(600 \mathrm{MHz}, \mathrm{CDCl}_{3}\right): \delta=7.55(\mathrm{t}, J=7.8 \mathrm{~Hz}, 1 \mathrm{H}, \mathrm{ArH}), 7.36(\mathrm{~d}, J=7.8 \mathrm{~Hz}, 2 \mathrm{H}, \mathrm{ArH}), 2.64-2.66(\mathrm{~m}$, $\left.2 \mathrm{H}, \mathrm{CCH}_{2} \mathrm{C}\right), 2.49-2.51\left(\mathrm{~m}, 2 \mathrm{H}, \mathrm{CCH}_{2} \mathrm{C}\right), 2.34-2.36\left(\mathrm{~m}, 2 \mathrm{H}, \mathrm{CCH}_{2} \mathrm{C}\right), 2.14-2.16(\mathrm{~m}, 2 \mathrm{H}, \mathrm{CCHC})$, $1.27\left(\mathrm{~d}, J=6.8 \mathrm{~Hz}, 6 \mathrm{H}, \mathrm{CCH}_{3}\right), 1.19\left(\mathrm{~d}, J=6.9 \mathrm{~Hz}, 6 \mathrm{H}, \mathrm{CCH}_{3}\right) ;{ }^{13} \mathrm{C}$ NMR $\left(150 \mathrm{MHz}, \mathrm{CDCl}_{3}\right): \delta=$ 191.9, 188.6, 155.4, 146.0, 131.7, 131.1, 125.0, 120.8, 120.8, 37.5, 29.0, 29.0, 25.6, 24.6, 24.6, 24.0, 24.0, 22.3; HRMS (TOF ES+): $\mathrm{m} / \mathrm{z}$ calcd for $\mathrm{C}_{19} \mathrm{H}_{24} \mathrm{NOS}_{2}\left[(\mathrm{M}+\mathrm{H})^{+}\right]$, 346.1294, found, 346.1296.

\section{3-(2,6-Diisopropylphenyl)-5,5-dimethyl-2-thioxo-2,3,5,6-tetrahydrobenzo[d]thiazol-7(4H)-one} (40)

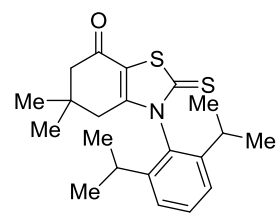

$\mathrm{V}_{\text {Petroleum ether }} / \mathrm{V}_{\text {Ethyl acetate }}=4: 1, \mathrm{R}_{f}=0.20$; Beige solid: $141 \mathrm{mg}(76 \%) ; \mathrm{mp}=190-192{ }^{\circ} \mathrm{C} ;{ }^{1} \mathrm{H}$ NMR $\left(600 \mathrm{MHz}, \mathrm{CDCl}_{3}\right): \delta=7.57(\mathrm{t}, J=7.8 \mathrm{~Hz}, 1 \mathrm{H}, \mathrm{ArH}), 7.37(\mathrm{~d}, J=7.8 \mathrm{~Hz}, 2 \mathrm{H}, \mathrm{ArH}), 2.51(\mathrm{~s}, 2 \mathrm{H}$, $\mathrm{CCH}_{2} \mathrm{C}$ ), 2.49-2.44 (m, 2H, CCHC), 2.21 (s, 2H, $\left.\mathrm{CCH}_{2} \mathrm{C}\right), 1.28$ (d, J=6.8 Hz, 6H, $\left.\mathrm{CCH}_{3}\right), 1.22$ (d, $\left.J=6.9 \mathrm{~Hz}, 6 \mathrm{H}, \mathrm{CCH}_{3}\right), 1.14\left(\mathrm{~s}, 6 \mathrm{H}, \mathrm{CCH}_{3}\right) ;{ }^{13} \mathrm{C} \mathrm{NMR}\left(150 \mathrm{MHz}, \mathrm{CDCl}_{3}\right): \delta=192.1,188.2,153.9$, 
146.1, 131.4, 131.1, 125.1, 125.1, 119.6, 51.5, 39.0, 35.0, 29.0, 29.0, 28.3, 28.3, 24.8, 24.8, 24.1, 24.1; HRMS (TOF ES+): m/z calcd for $\mathrm{C}_{21} \mathrm{H}_{28} \mathrm{NOS}_{2}\left[(\mathrm{M}+\mathrm{H})^{+}\right]$, 374.1607, found, 374.1607.

3-Mesityl-2-thioxo-2,3,5,6-tetrahydrobenzo[d]thiazol-7(4H)-one (4p)

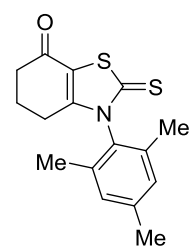

$\mathrm{V}_{\text {Petroleum ether }} / \mathrm{V}_{\text {Ethyl acetate }}=5: 1, \mathrm{R}_{f}=0.20$; Beige solid: $111 \mathrm{mg}(73 \%) ; \mathrm{mp}=215-217{ }^{\circ} \mathrm{C} ;{ }^{1} \mathrm{H}$ NMR $\left(600 \mathrm{MHz}, \mathrm{CDCl}_{3}\right): \delta=7.06(\mathrm{~s}, 2 \mathrm{H}, \mathrm{ArH}), 2.64-2.66\left(\mathrm{~m}, 2 \mathrm{H}, \mathrm{CCH}_{2} \mathrm{C}\right), 2.38\left(\mathrm{~s}, 3 \mathrm{H}, \mathrm{ArCH}_{3}\right)$, 2.33-2.35 (m, $\left.2 \mathrm{H}, \mathrm{CCH}_{2} \mathrm{C}\right), 2.17-2.19\left(\mathrm{~m}, 2 \mathrm{H}, \mathrm{CCH}_{2} \mathrm{C}\right), 2.08\left(\mathrm{~s}, 6 \mathrm{H}, \mathrm{ArCH}_{3}\right) ;{ }^{13} \mathrm{C} \mathrm{NMR}(150 \mathrm{MHz}$, $\left.\mathrm{CDCl}_{3}\right): \delta=190.7,188.6,154.7,140.5,135.2,132.0,129.9,129.9,120.9,37.5,24.9,22.3,21.3$, 17.6, 17.6; HRMS (TOF ES+): $\mathrm{m} / \mathrm{z}$ calcd for $\mathrm{C}_{16} \mathrm{H}_{18} \mathrm{NOS}_{2}\left[(\mathrm{M}+\mathrm{H})^{+}\right]$, 304.0824, found, 304.0821.

\section{3-Mesityl-5,5-dimethyl-2-thioxo-2,3,5,6-tetrahydrobenzo[d]thiazol-7(4H)-one (4q)}

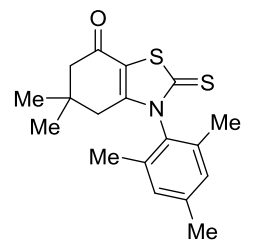

$\mathrm{V}_{\text {Petroleum ether }} / \mathrm{V}_{\text {Ethyl acetate }}=5: 1, \mathrm{R}_{f}=0.20$; Beige solid: $124 \mathrm{mg}(75 \%) ; \mathrm{mp}=187-189{ }^{\circ} \mathrm{C} ;{ }^{1} \mathrm{H}$ NMR (600 MHz, $\left.\mathrm{CDCl}_{3}\right): \delta=7.08(\mathrm{~s}, 2 \mathrm{H}, \mathrm{ArH}), 2.51\left(\mathrm{~s}, 2 \mathrm{H}, \mathrm{CCH}_{2}\right), 2.39$ (s, 3H, $\left.\mathrm{ArCH}_{3}\right), 2.20(\mathrm{~s}, 2 \mathrm{H}$, $\mathrm{CCH}_{2} \mathrm{C}$ ), 2.07 (s, 6H, $\left.\mathrm{ArCH}_{3}\right), 1.14$ (s, 6H, $\left.\mathrm{CCH}_{3}\right) ;{ }^{13} \mathrm{C} \mathrm{NMR}\left(150 \mathrm{MHz}, \mathrm{CDCl}_{3}\right): \delta=190.9,188.2$, 153.5, 140.5, 135.1, 132.0, 130.0, 119.6, 119.6, 51.4, 38.6, 34.9, 28.3, 28.3, 21.3, 17.6, 17.6; HRMS (TOF ES $\left.{ }^{+}\right): \mathrm{m} / \mathrm{z}$ calcd for $\mathrm{C}_{18} \mathrm{H}_{22} \mathrm{NOS}_{2}\left[(\mathrm{M}+\mathrm{H})^{+}\right], 332.1137$, found, 332.1138 .

3-Methylbenzo $[d]$ thiazole-2(3H)-thione $(7 \mathbf{a})^{3}$

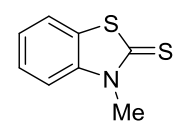

$\mathrm{V}_{\text {Petroleum ether }} / \mathrm{V}_{\text {Ethyl acetate }}=1: 1, \mathrm{R}_{f}=0.30 ;$ White solid: $58 \mathrm{mg}(64 \%) ; \mathrm{mp}=85-87{ }^{\circ} \mathrm{C} ;{ }^{1} \mathrm{H}$ NMR $(600$ MHz, DMSO- $\left.d_{6}\right): \delta=7.78-7.79$ (d, $\left.J=7.9 \mathrm{~Hz}, 1 \mathrm{H}, \mathrm{ArH}\right), 7.50-7.57(\mathrm{~m}, 2 \mathrm{H}, \mathrm{ArH}), 7.37-7.40(\mathrm{~m}$, $1 \mathrm{H}, \mathrm{ArH}), 3.82\left(\mathrm{~s}, 3 \mathrm{H}, \mathrm{NCH}_{3}\right) ;{ }^{13} \mathrm{C} \mathrm{NMR}\left(150 \mathrm{MHz}, \mathrm{DMSO}-d_{6}\right): \delta=188.5,142.4,127.8,126.8$, 125.5, 122.3, 114.0, 33.7; HRMS (TOF ES ${ }^{+}$): $\mathrm{m} / \mathrm{z}$ calcd for $\mathrm{C}_{8} \mathrm{H}_{8} \mathrm{NS}_{2}\left[(\mathrm{M}+\mathrm{H})^{+}\right], 182.0093$, found, 182.0095 .

\section{3-Benzylbenzo $[d]$ thiazole-2(3H)-thione $(7 b)^{3}$}

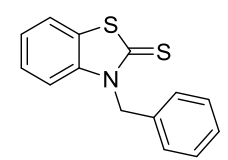

$\mathrm{V}_{\text {Petroleum ether }} / \mathrm{V}_{\text {Ethyl acetate }}=2: 1, \mathrm{R}_{f}=0.30$; White solid: $114 \mathrm{mg}(89 \%) ; \mathrm{mp}=144-145{ }^{\circ} \mathrm{C} ;{ }^{1} \mathrm{H}$ NMR (600 MHz, DMSO- $\left.d_{6}\right): \delta=7.80-7.82(\mathrm{~d}, J=7.8 \mathrm{~Hz}, 1 \mathrm{H}, \mathrm{ArH}), 7.26-7.43(\mathrm{~m}, 8 \mathrm{H}, \mathrm{ArH}), 5.75(\mathrm{~s}, 2 \mathrm{H}$, $\left.\mathrm{NCH}_{2} \mathrm{C}\right) ;{ }^{13} \mathrm{C}$ NMR $\left(150 \mathrm{MHz}, \mathrm{DMSO}-d_{6}\right): \delta=189.5,141.5,135.3,129.3,129.3,128.2,127.8$, 
127.5, 127.5, 127.0, 125.6, 122.5, 114.2, 48.9; HRMS (TOF ES $\left.{ }^{+}\right): \mathrm{m} / \mathrm{z}$ calcd for $\mathrm{C}_{14} \mathrm{H}_{12} \mathrm{NS}_{2}$ $\left[(\mathrm{M}+\mathrm{H})^{+}\right], 258.0406$, found, 258.0410 . 


\section{X-ray Structure and Data ${ }^{4}$ of 2a (CCDC 2062625).}

Single crystal culture and confirmation: First, compound 2a was added to a bottle and dissolved by the addition of dichloromethane and ethyl acetate. Then, the bottle was placed in a jar, and petroleum ether $(15 \mathrm{~mL})$ was added to the bottle. The jar was sealed, and the bottle within the jar remained at room temperature for 3 days. Some crystals appeared, and for single crystal parsing. The APEX DUO diffractometer was used to obtain single crystal diffraction at 293(2) K with the use of four-circle diffractometer Mo K (lambda $=1.54184$ A) for diffraction intensity data collection, using omega scanning. Multi-scan correction was performed for all intensity data. The crystal structure was solved by the atomic method using the SHELXT program (Supporting Information, Figure S1, CCDC 2062625).

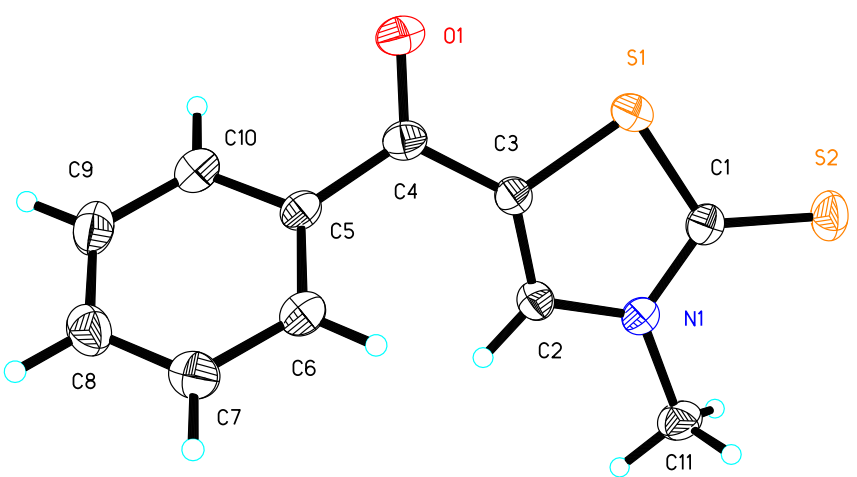

Figure S1 X-Ray crystal structure of 2a, ellipsoids are drawn at the 30\% probability level.

Table S1 Crystal data and structure refinement for $2 \mathbf{a}$.

Empirical formula

Formula weight

Temperature

Wavelength

Crystal system, space group

Unit cell dimensions

Volume

Z, Calculated density

Absorption coefficient

$\mathrm{F}(000)$

Theta range for data collection

Limiting indices

Completeness to theta $=66.97$

Absorption correction
$\mathrm{C}_{11} \mathrm{H}_{9} \mathrm{NOS}_{2}$

235.31

$293.15 \mathrm{~K}$

$1.54184 \AA$

Orthorhombic, P 1 21/c 1

$\mathrm{a}=9.4254(9) \mathrm{A} \quad$ alpha $=90 \mathrm{deg}$.

$\mathrm{b}=10.3437(13) \mathrm{A} \quad$ beta $=104.385(10) \mathrm{deg}$.

$\mathrm{c}=11.5166(11) \mathrm{A} \quad$ gamma $=90 \mathrm{deg}$.

$1087.6(2) \mathrm{A}^{\wedge} 3$

$4, \quad 1.437 \mathrm{Mg} / \mathrm{m}^{\wedge} 3$

$4.197 \mathrm{~mm}^{\wedge}-1$

488

4.2420 to $69.6170 \mathrm{deg}$.

$-11<=\mathrm{h}<=8,-12<=\mathrm{k}<=8,-13<=\mathrm{l}<=12$

$99.13 \%$

Multi-scan 
Refinement method

Data/restraints/parameters

Goodness-of-fit on $\mathrm{F}^{\wedge} 2$

Final R indices [I>2sigma(I)]

$\mathrm{R}$ indices (all data)

Largest diff. peak and hole
SHELXL-2018/3 (Sheldrick 2018)

2040 / 0 / 138

1.068

$\mathrm{R} 1=0.0502, \mathrm{wR} 2=0.1234$

$\mathrm{R} 1=0.0670, \mathrm{wR} 2=0.1404$

0.354 and -0.286 e. $\mathrm{A}^{\wedge}-3$ 
5. ${ }^{1} \mathrm{H}$ NMR and ${ }^{13} \mathrm{C}$ NMR spectra for spectroscopic data. 

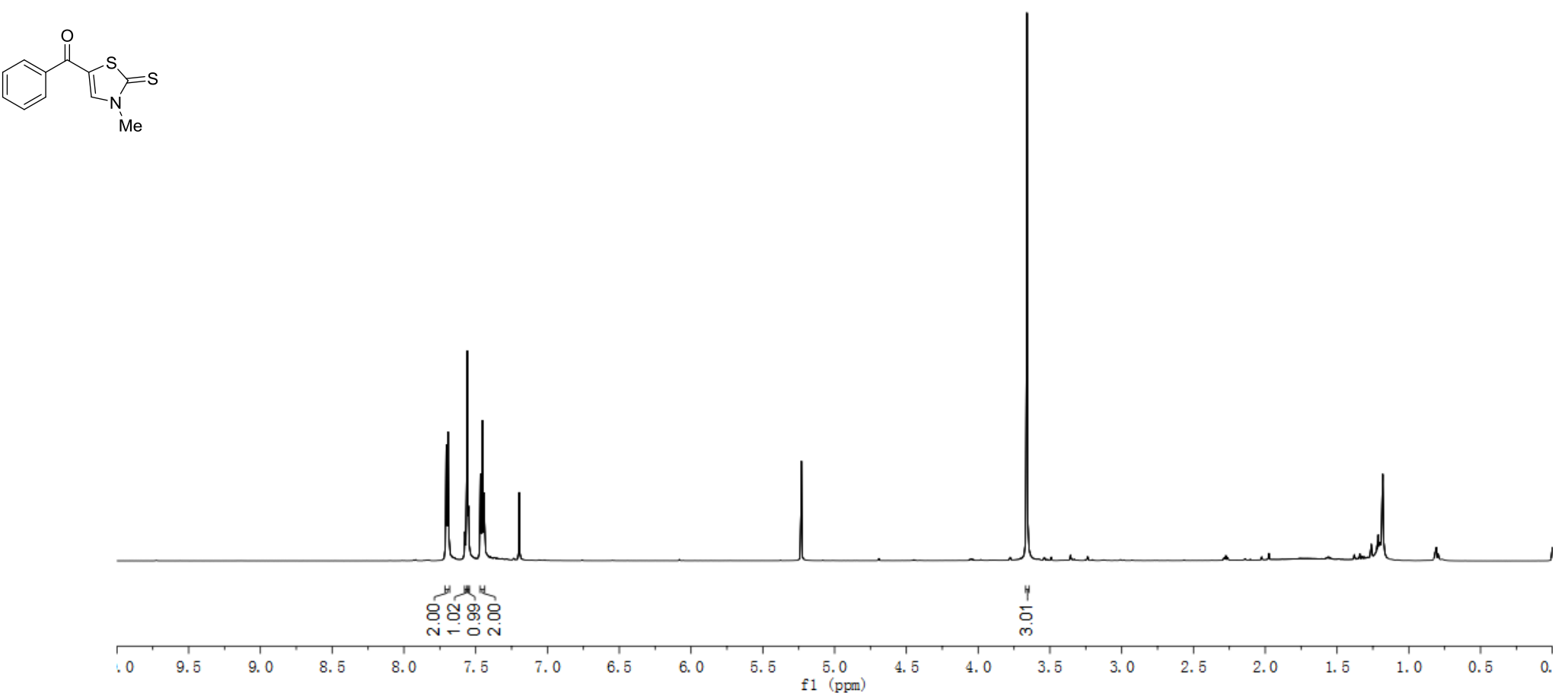

Figure S2. $\quad{ }^{1} \mathrm{H} \mathrm{NMR}\left(600 \mathrm{MHz}, \mathrm{CDCl}_{3}\right)$ spectra of compound 2a 
亳融

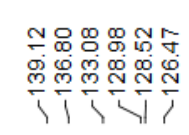

盟量

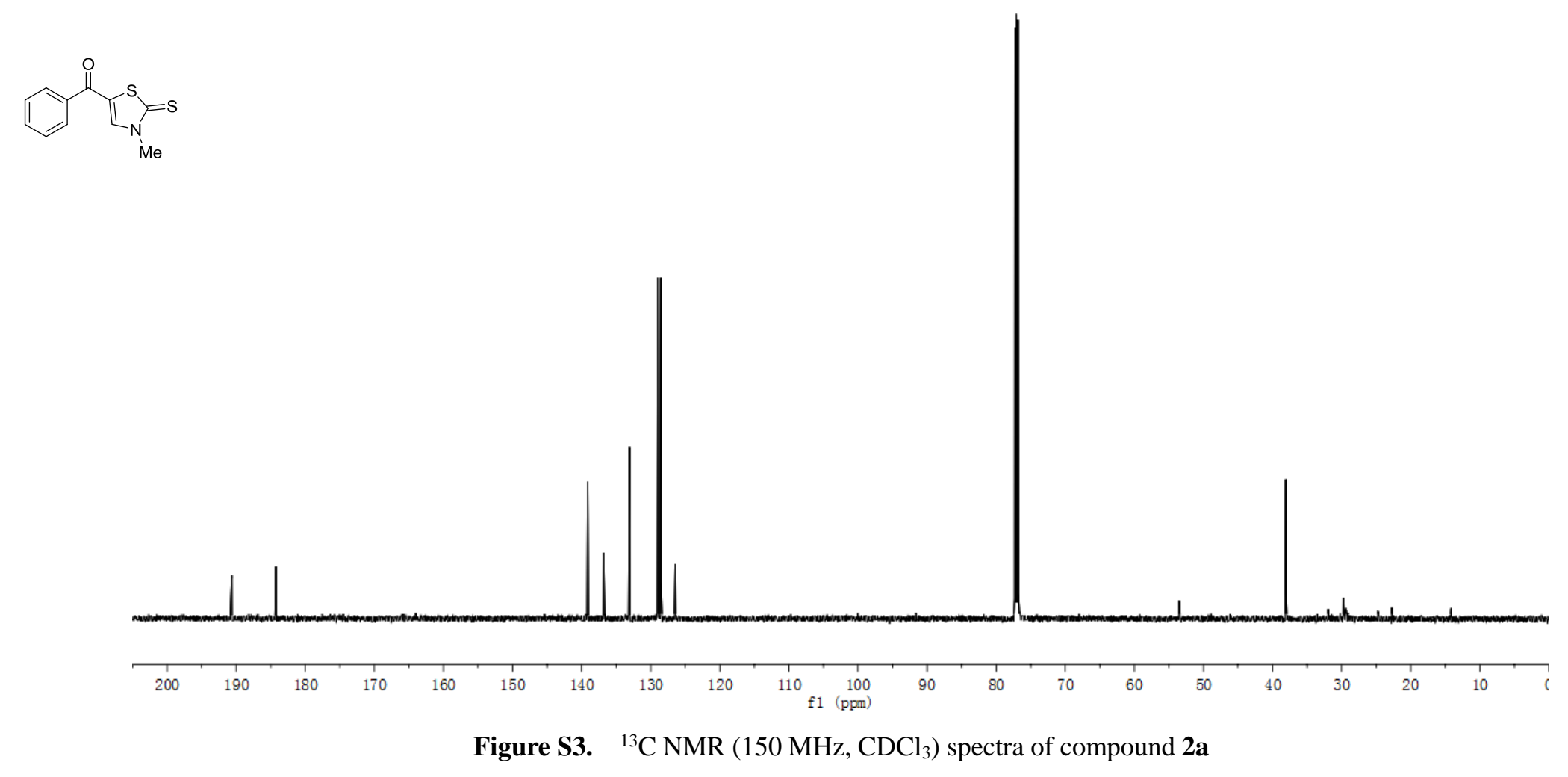




$$
{ }^{2}
$$




\begin{tabular}{|c|c|c|c|}
\hline $\begin{array}{ll}0 \\
0 \\
0 \\
0 \\
0 \\
0 \\
& 0 \\
& 0 \\
\end{array}$ & 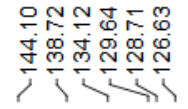 & 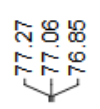 & $\begin{array}{l}n \\
\infty \\
\infty \\
\infty \\
0 \\
1\end{array}$ \\
\hline
\end{tabular}

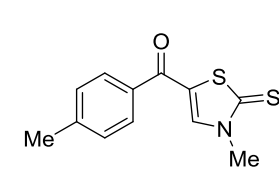

Me

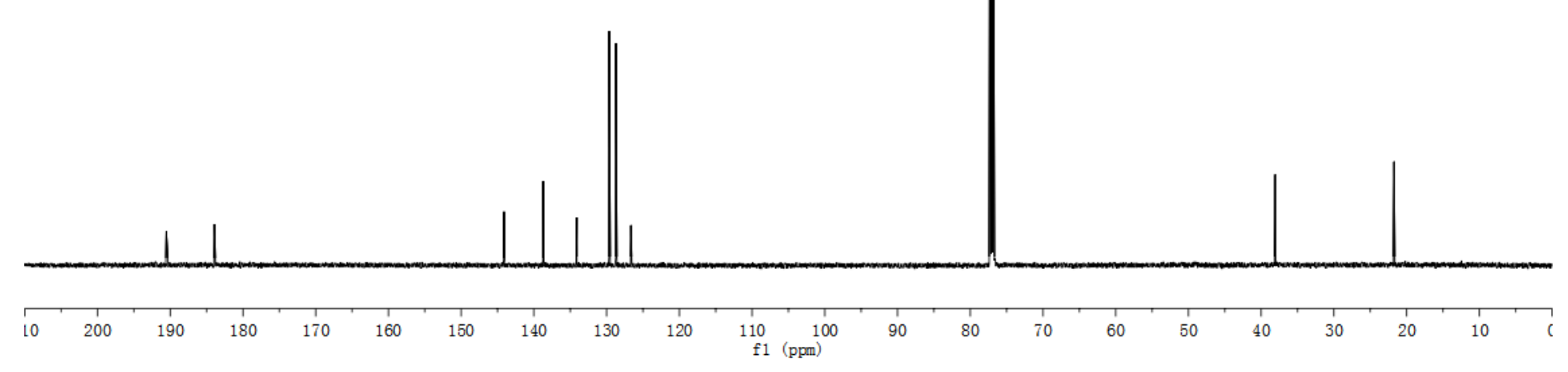

Figure S5. ${ }^{13} \mathrm{C} \mathrm{NMR}\left(150 \mathrm{MHz}, \mathrm{CDCl}_{3}\right)$ spectra of compound $\mathbf{2 b}$ 

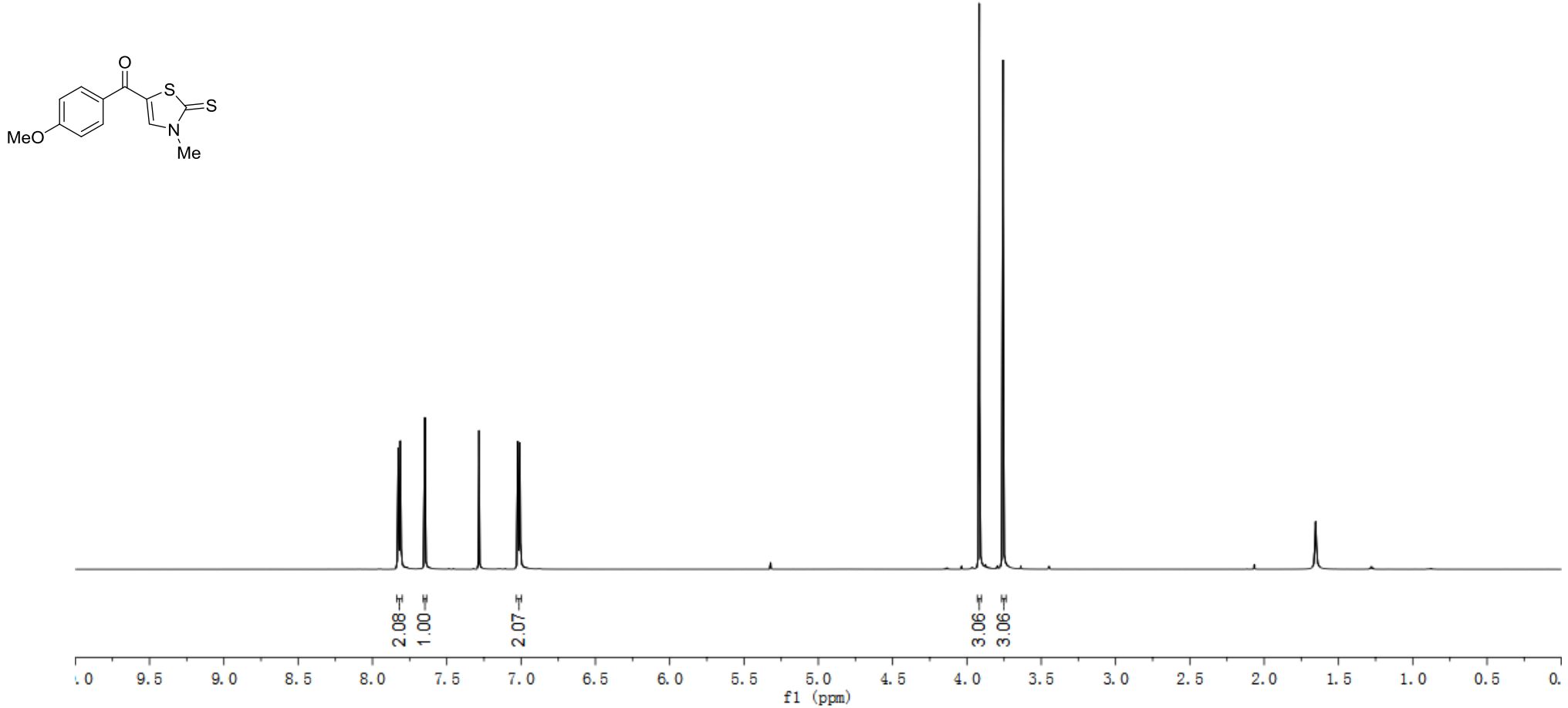

Figure S6. ${ }^{1} \mathrm{H} \mathrm{NMR}\left(600 \mathrm{MHz}, \mathrm{CDCl}_{3}\right)$ spectra of compound $\mathbf{2 c}$ 
I

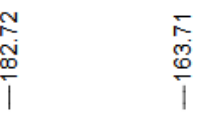

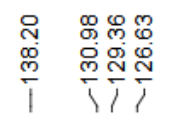

$\stackrel{\stackrel{m}{+}}{\frac{1}{+}}$

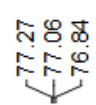

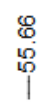

$\stackrel{\substack{\infty \\ \infty}}{\infty}$
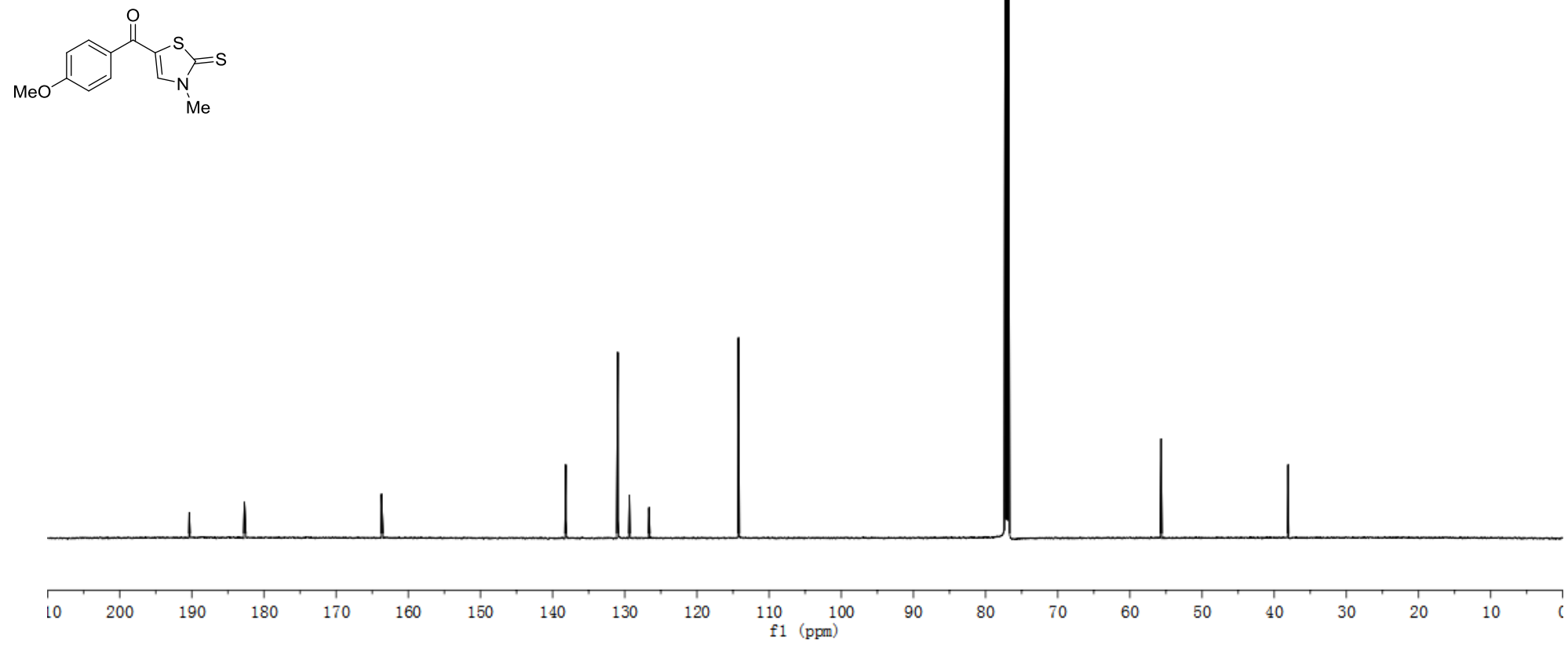

Figure S7. ${ }^{13} \mathrm{C} \mathrm{NMR}\left(150 \mathrm{MHz}, \mathrm{CDCl}_{3}\right)$ spectra of compound $2 \mathrm{c}$

S27 

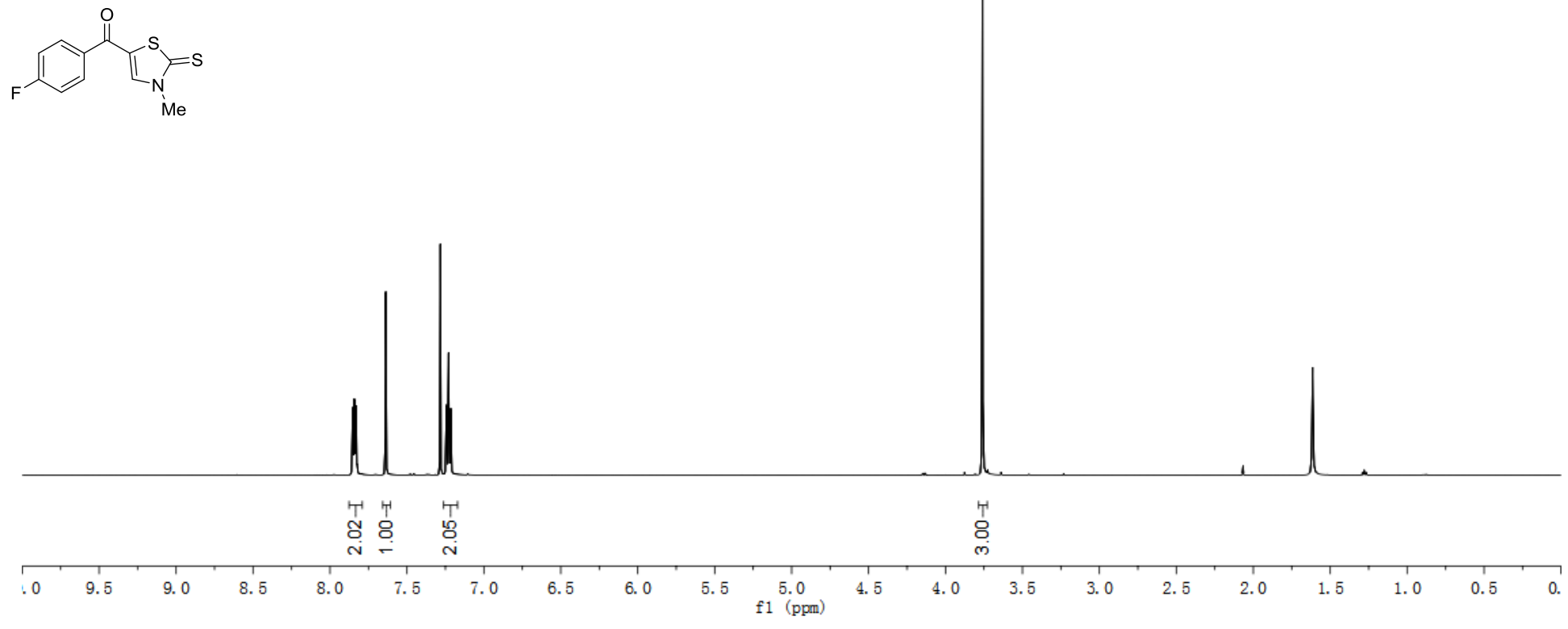

Figure S8. $\quad{ }^{1} \mathrm{H} \mathrm{NMR}\left(600 \mathrm{MHz}, \mathrm{CDCl}_{3}\right)$ spectra of compound $\mathbf{2 d}$ 


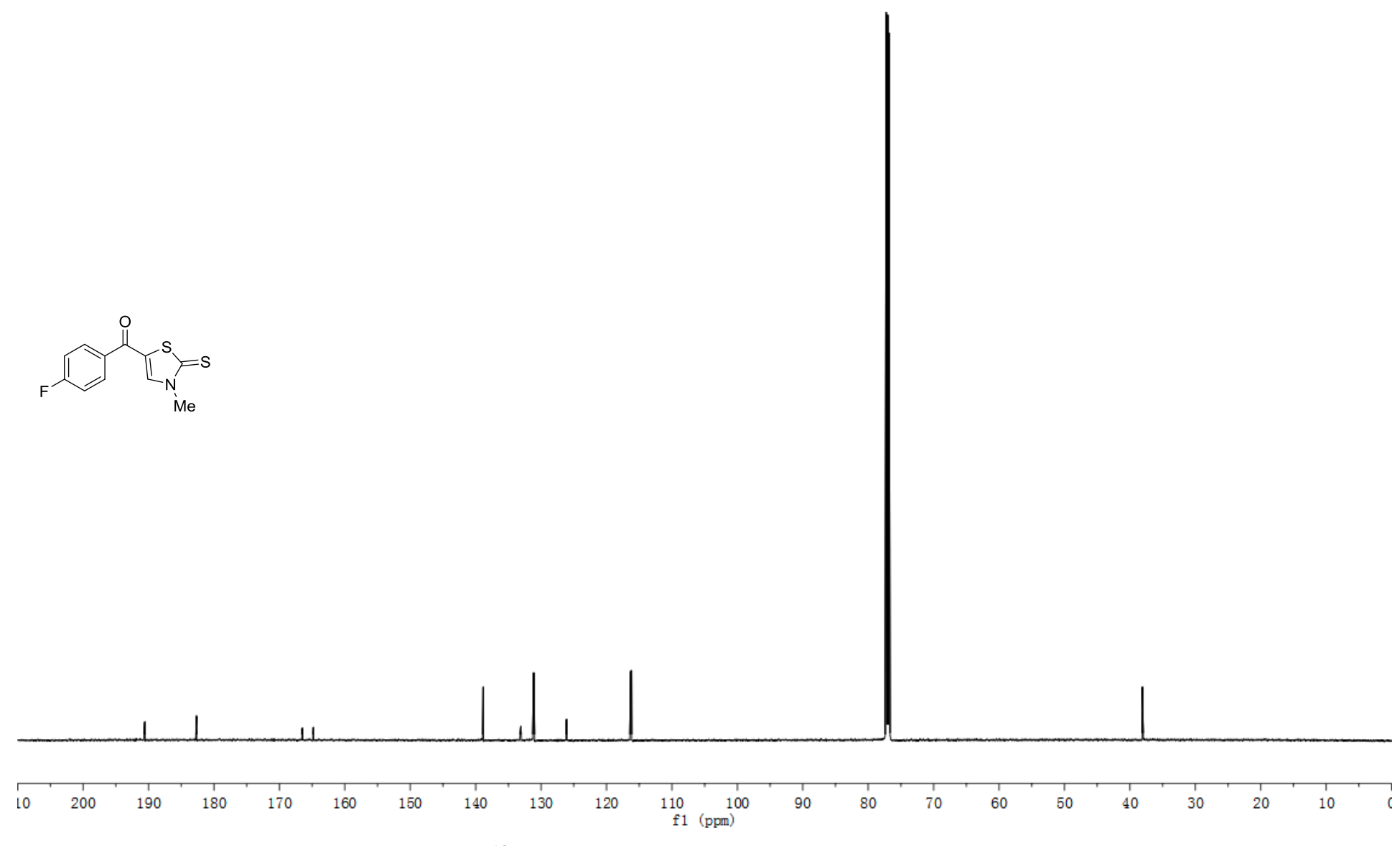

Figure S9. ${ }^{13} \mathrm{C} \mathrm{NMR}\left(150 \mathrm{MHz}, \mathrm{CDCl}_{3}\right)$ spectra of compound $\mathbf{2 d}$ 

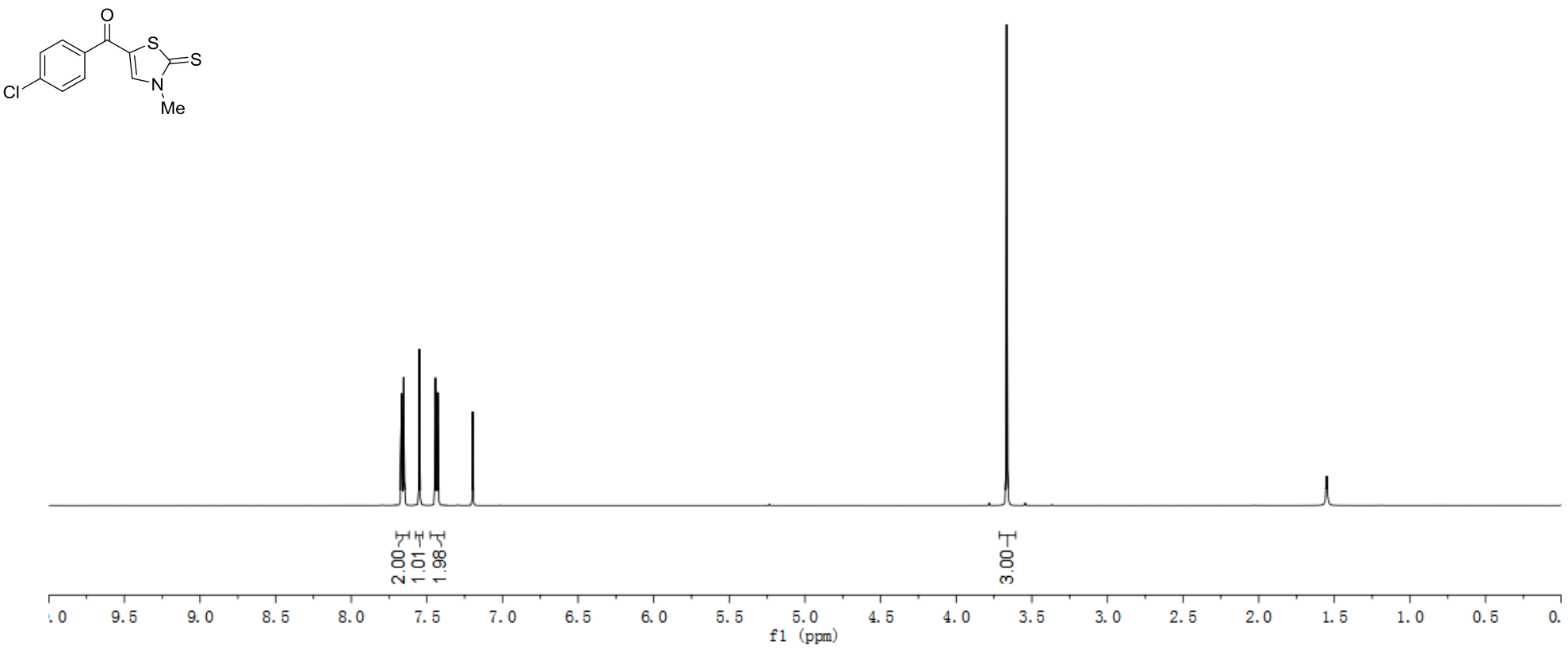

Figure S10. ${ }^{1} \mathrm{H} \mathrm{NMR}\left(600 \mathrm{MHz}, \mathrm{CDCl}_{3}\right)$ spectra of compound $2 \mathbf{e}$ 


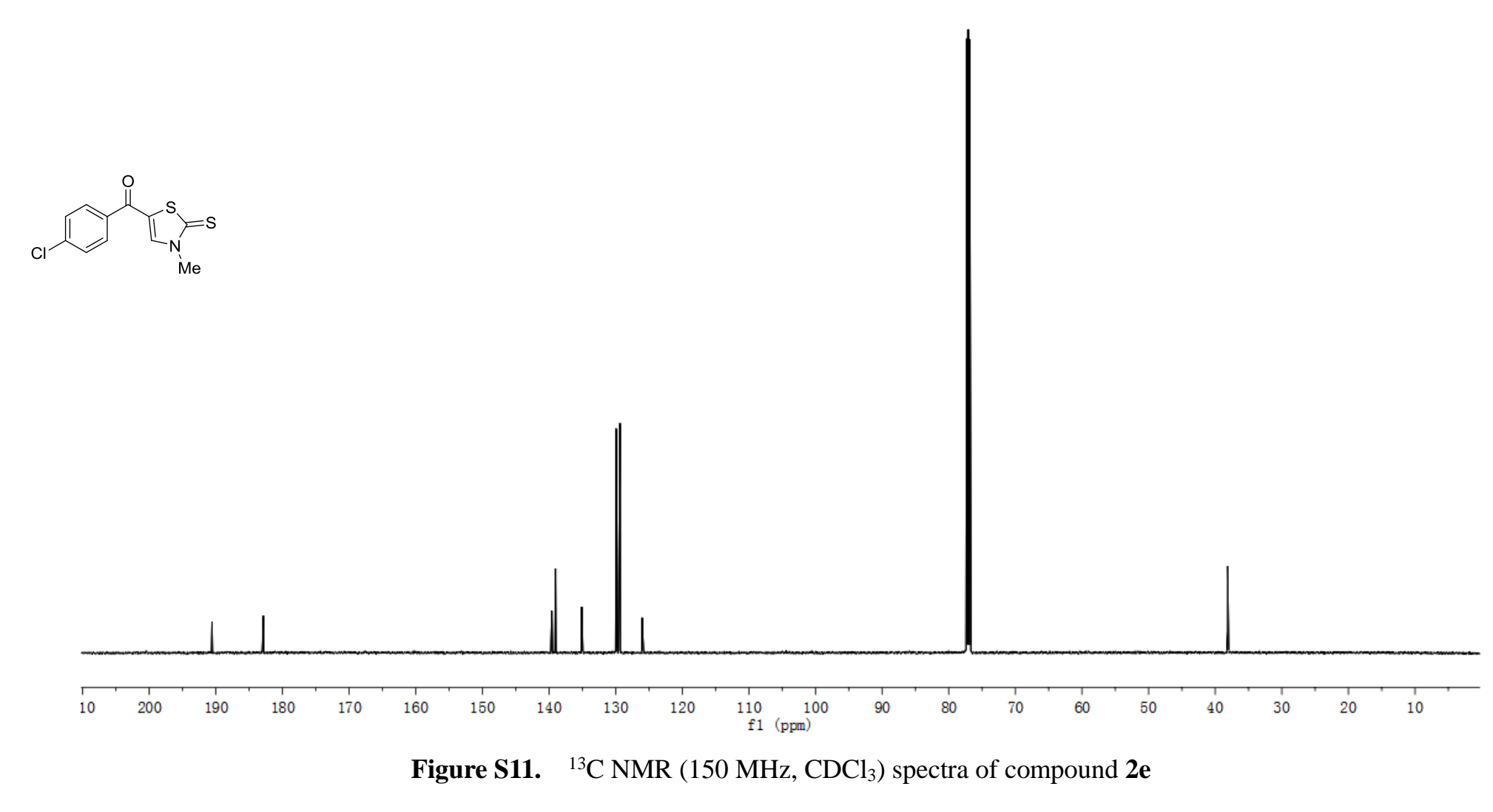



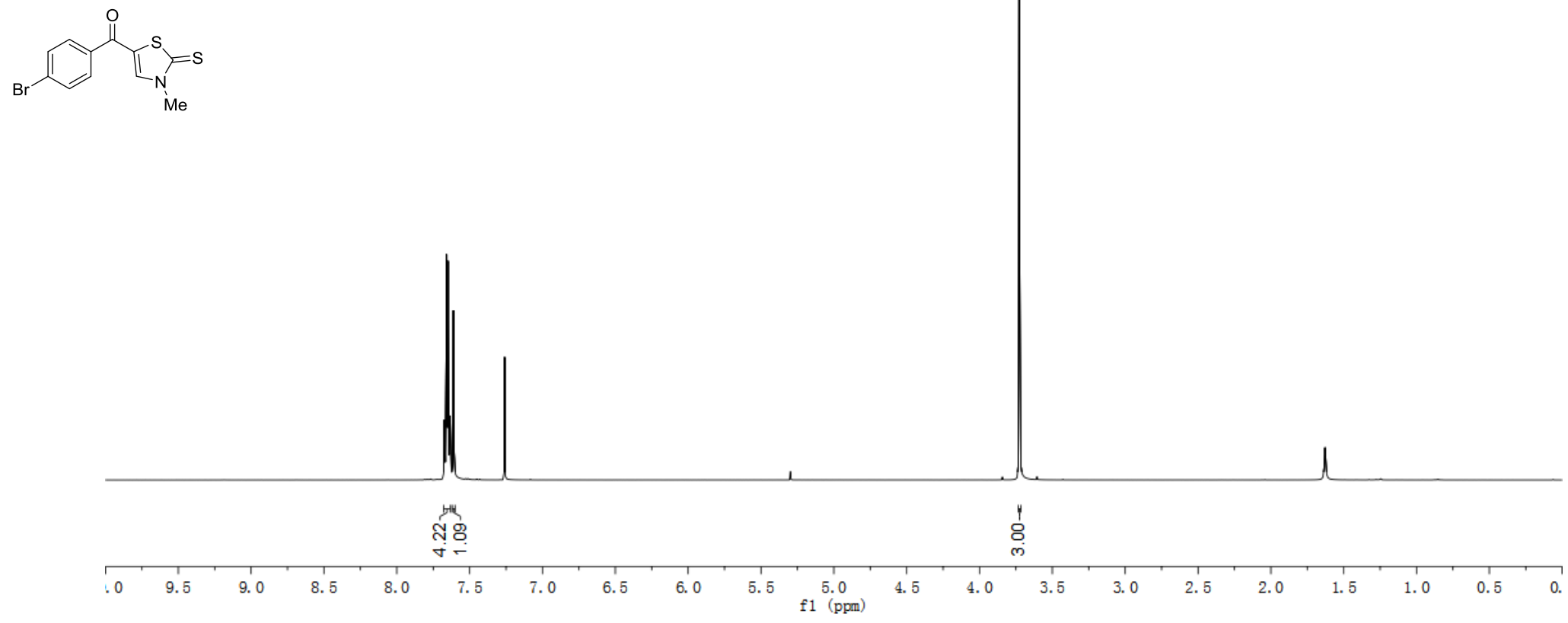

Figure S12. $\quad{ }^{1} \mathrm{H} \mathrm{NMR}\left(600 \mathrm{MHz}, \mathrm{CDCl}_{3}\right)$ spectra of compound $\mathbf{2 f}$ 


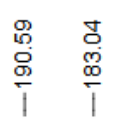

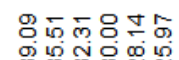
$\stackrel{1}{\sim}$
กิ้อ

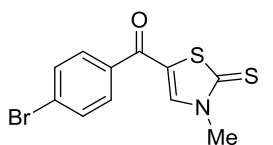

Me

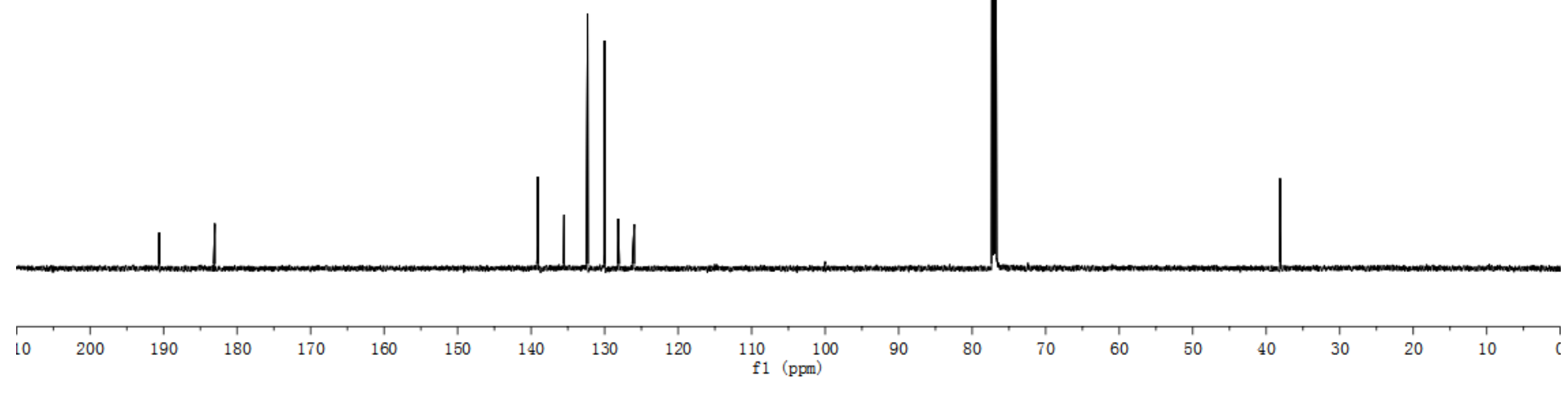

Figure S13. ${ }^{13} \mathrm{C}$ NMR $\left(150 \mathrm{MHz}, \mathrm{CDCl}_{3}\right)$ spectra of compound $\mathbf{2 f}$ 

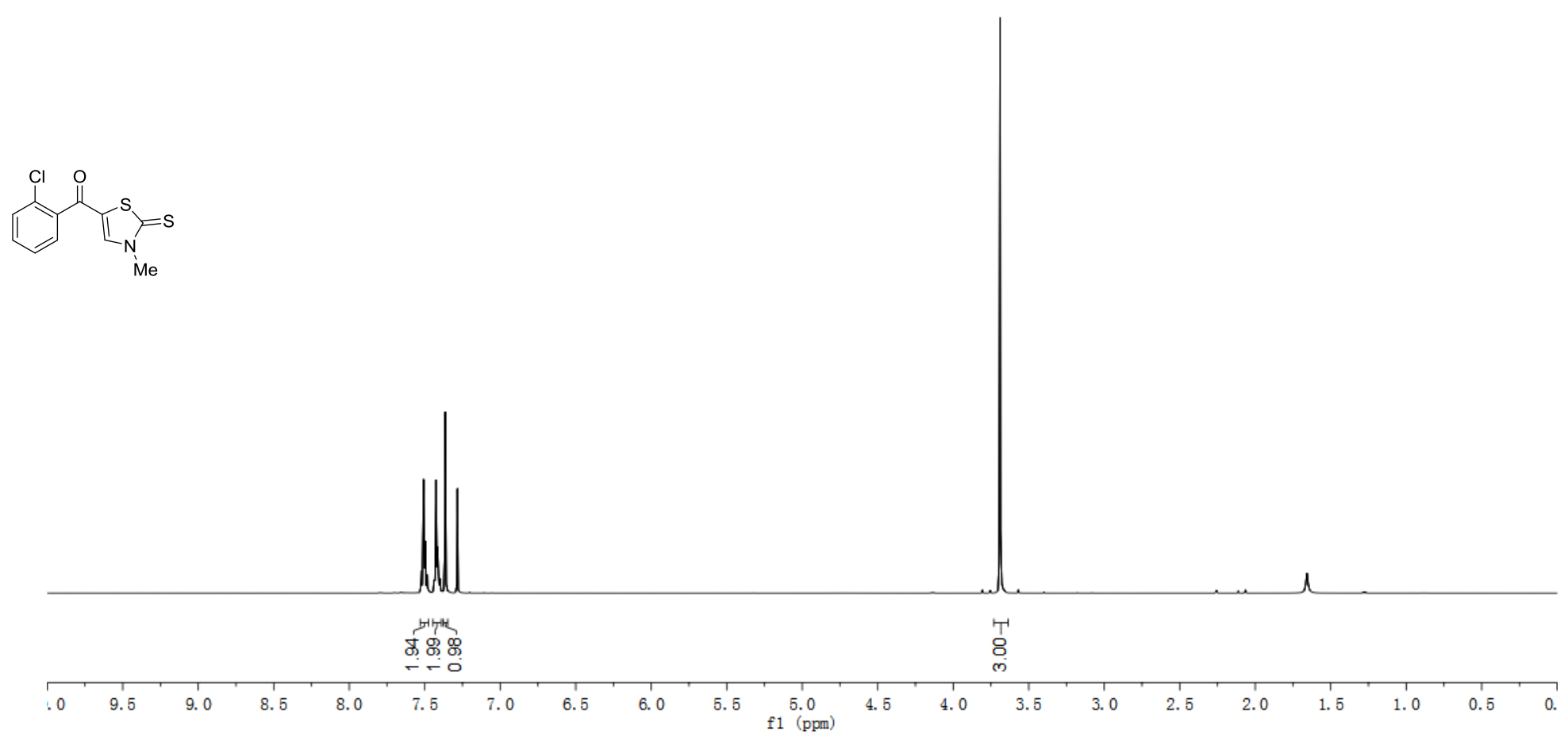

Figure S14. $\quad{ }^{1} \mathrm{H} \mathrm{NMR}\left(600 \mathrm{MHz}, \mathrm{CDCl}_{3}\right)$ spectra of compound $\mathbf{2 g}$ 


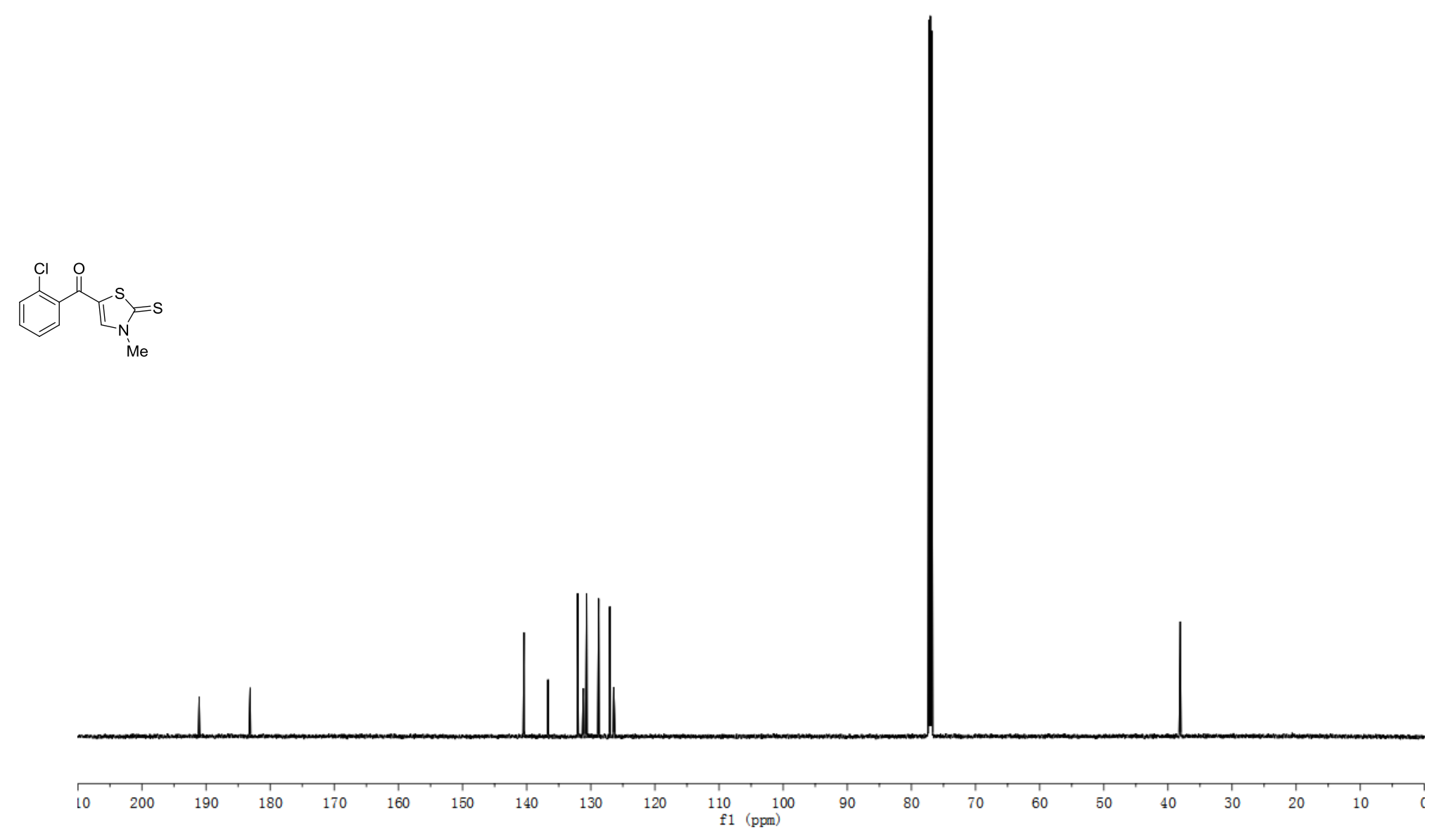

Figure S15. ${ }^{13} \mathrm{C}$ NMR $\left(150 \mathrm{MHz}, \mathrm{CDCl}_{3}\right)$ spectra of compound $\mathbf{2 g}$ 

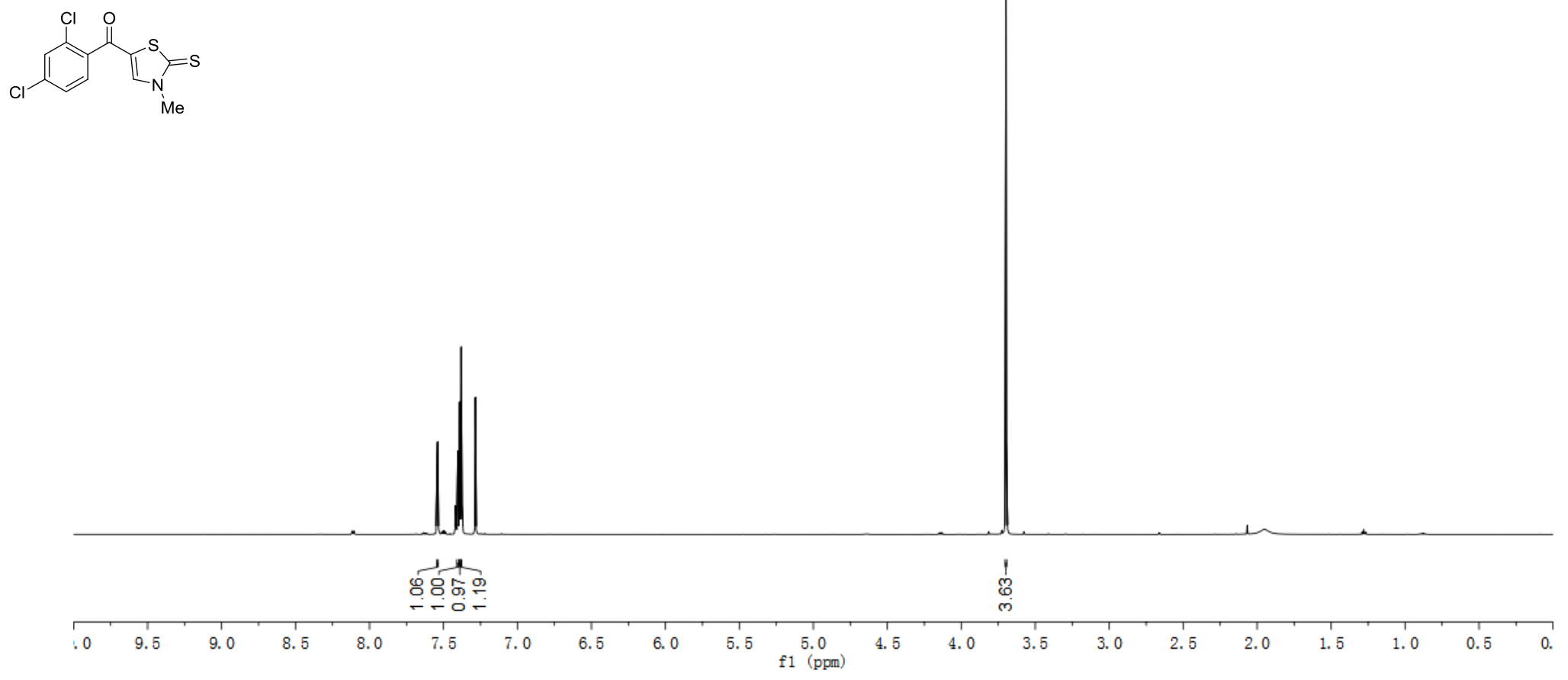

Figure S16. ${ }^{1} \mathrm{H} \mathrm{NMR}\left(600 \mathrm{MHz}, \mathrm{CDCl}_{3}\right)$ spectra of compound $\mathbf{2 h}$ 


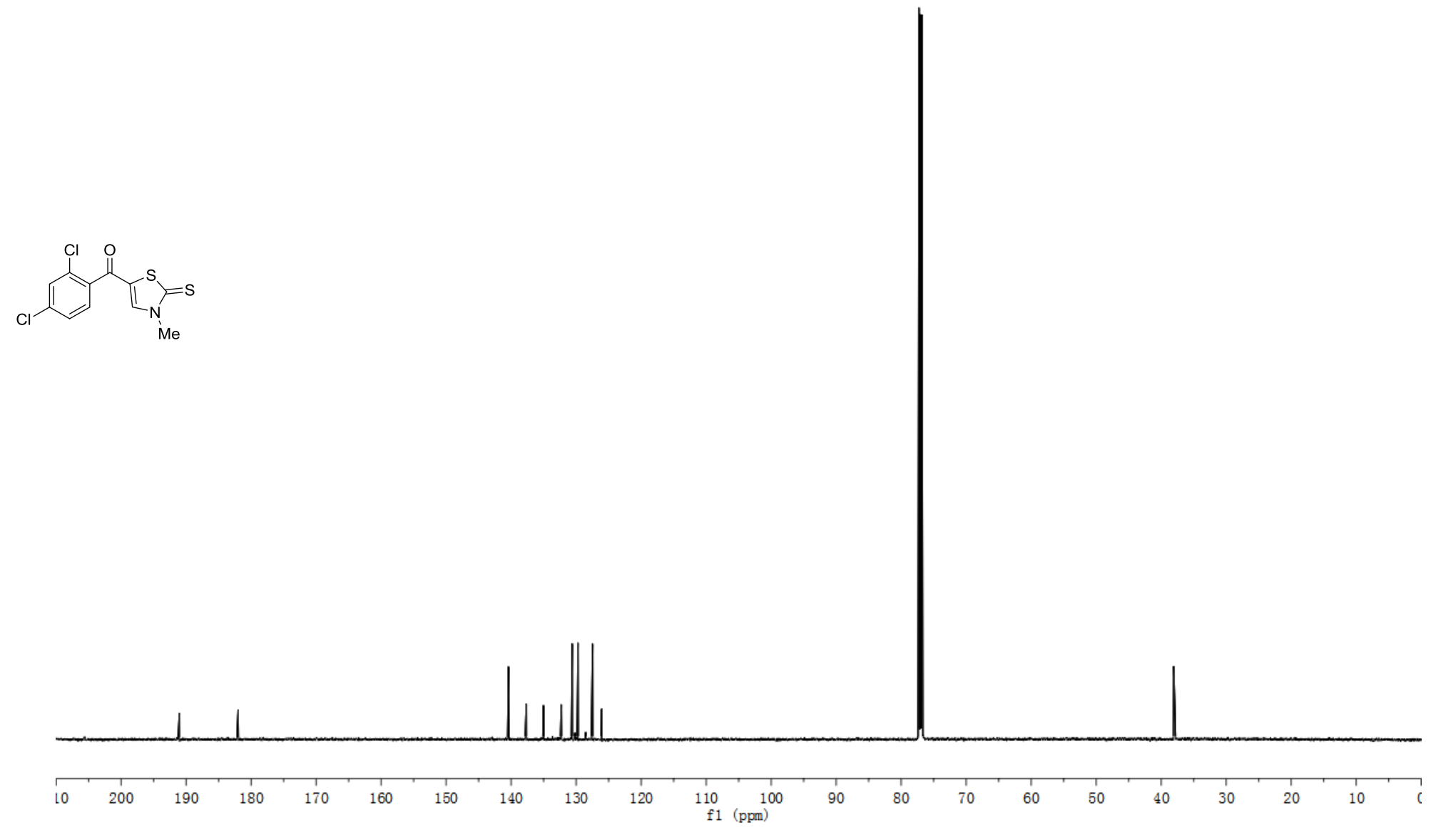

Figure S17. ${ }^{13} \mathrm{C} \mathrm{NMR}\left(150 \mathrm{MHz}, \mathrm{CDCl}_{3}\right)$ spectra of compound $\mathbf{2 h}$ 

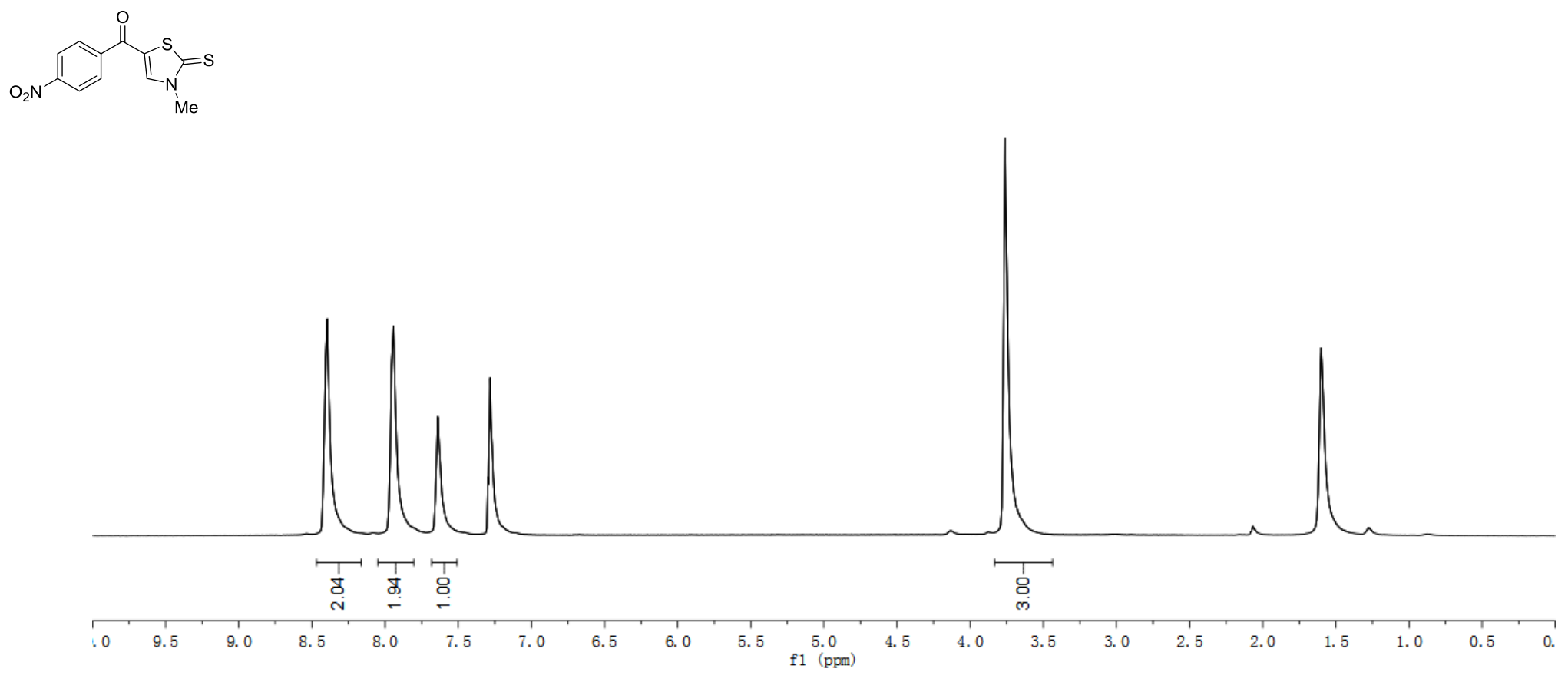

Figure S18. ${ }^{1} \mathrm{H}$ NMR $\left(600 \mathrm{MHz}, \mathrm{CDCl}_{3}\right)$ spectra of compound $\mathbf{2 i}$ 


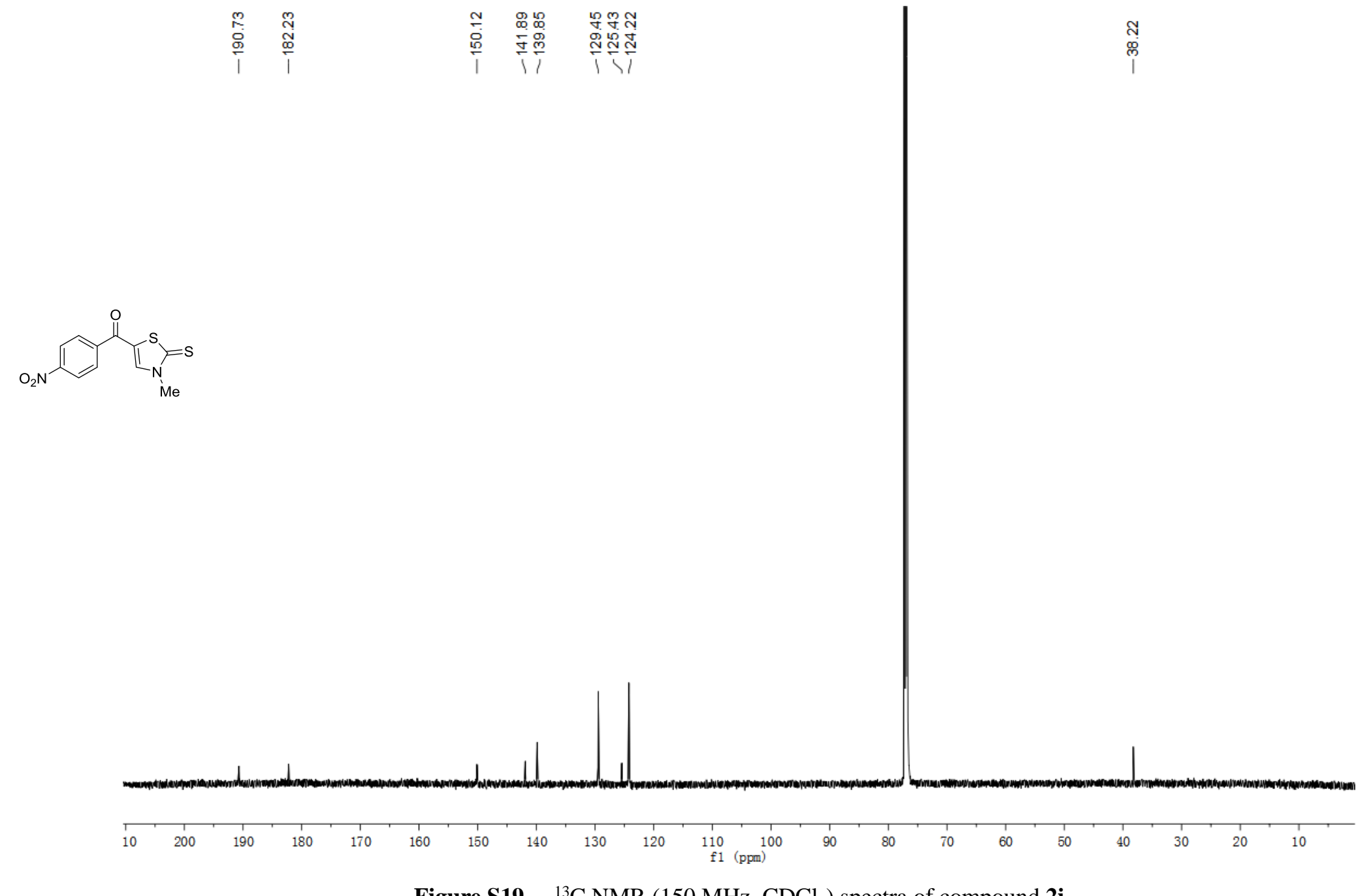

Figure S19. ${ }^{13} \mathrm{C} \mathrm{NMR}\left(150 \mathrm{MHz}, \mathrm{CDCl}_{3}\right)$ spectra of compound $\mathbf{2 i}$ 


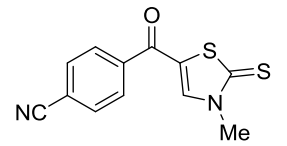

Me

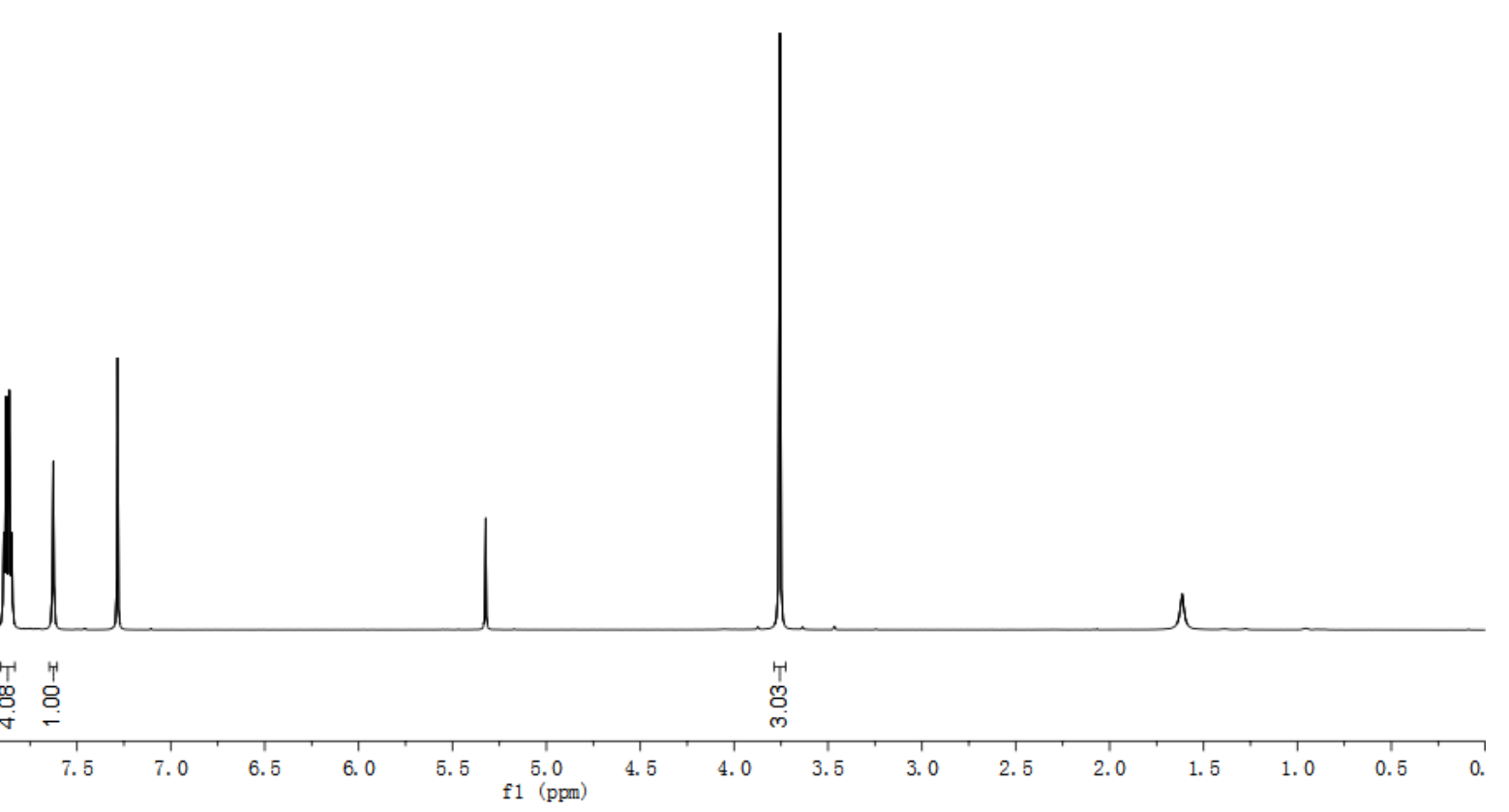

Figure S20. $\quad{ }^{1} \mathrm{H}$ NMR $\left(600 \mathrm{MHz}, \mathrm{CDCl}_{3}\right)$ spectra of compound $\mathbf{2 j}$ 


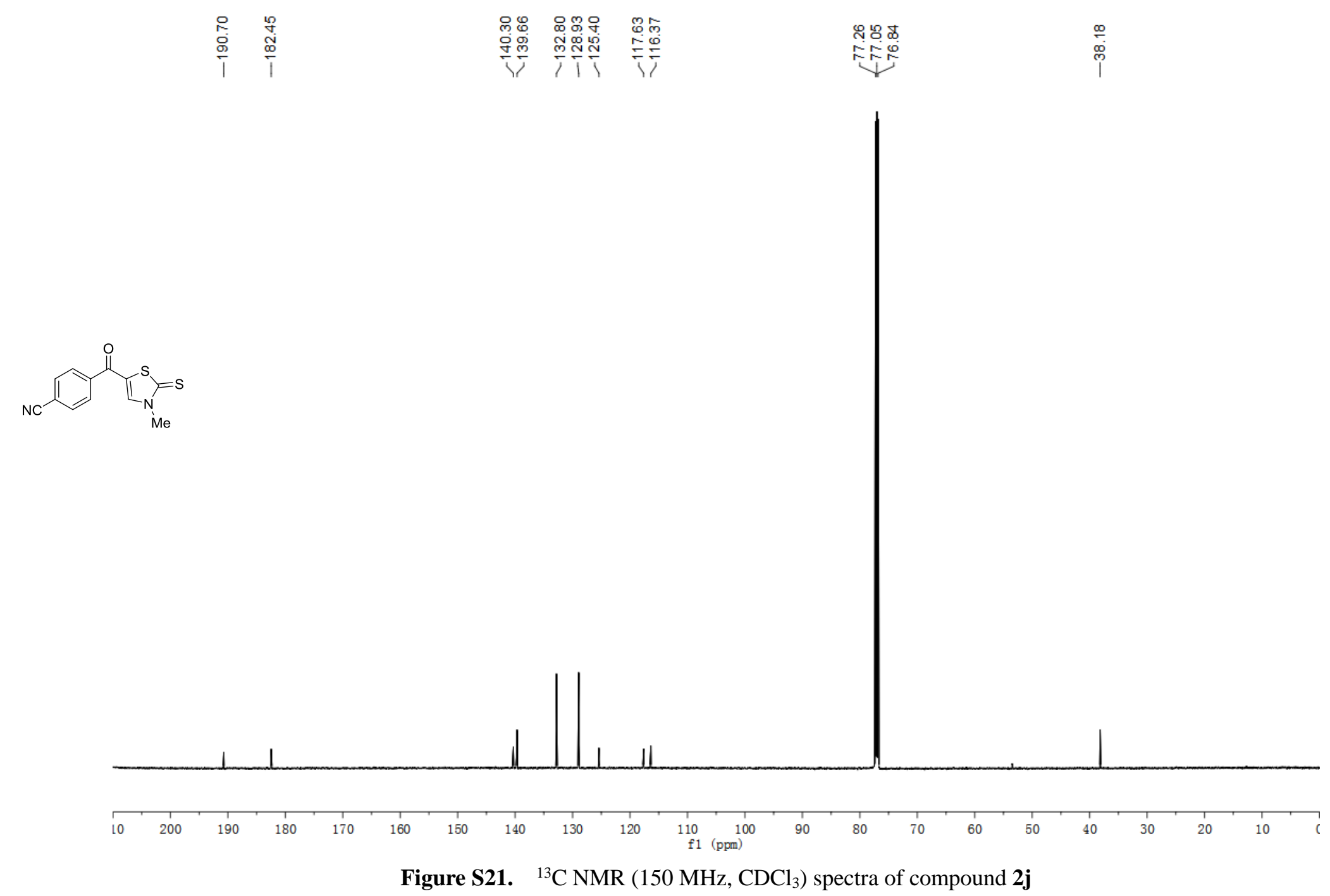



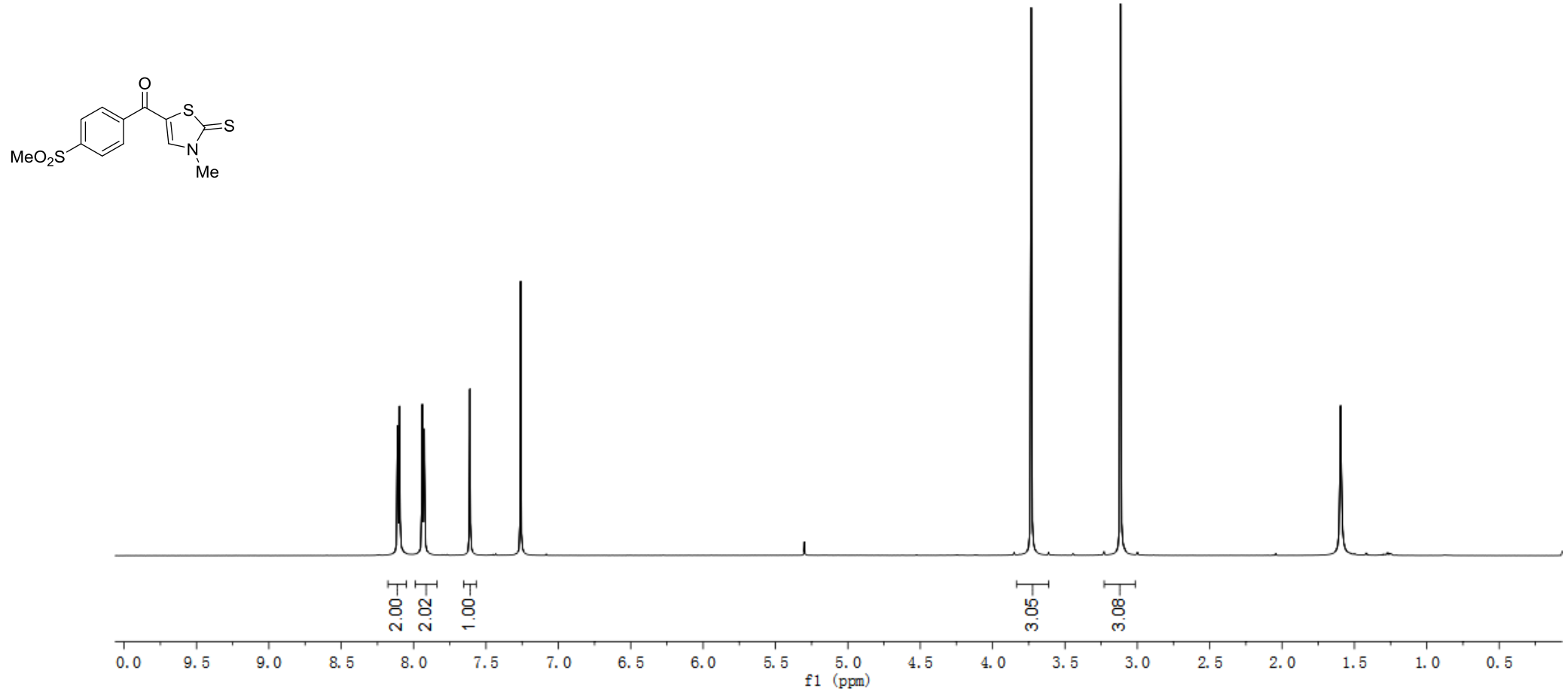

Figure S22. $\quad{ }^{1} \mathrm{H}$ NMR $\left(600 \mathrm{MHz}, \mathrm{CDCl}_{3}\right)$ spectra of compound $\mathbf{2 k}$ 


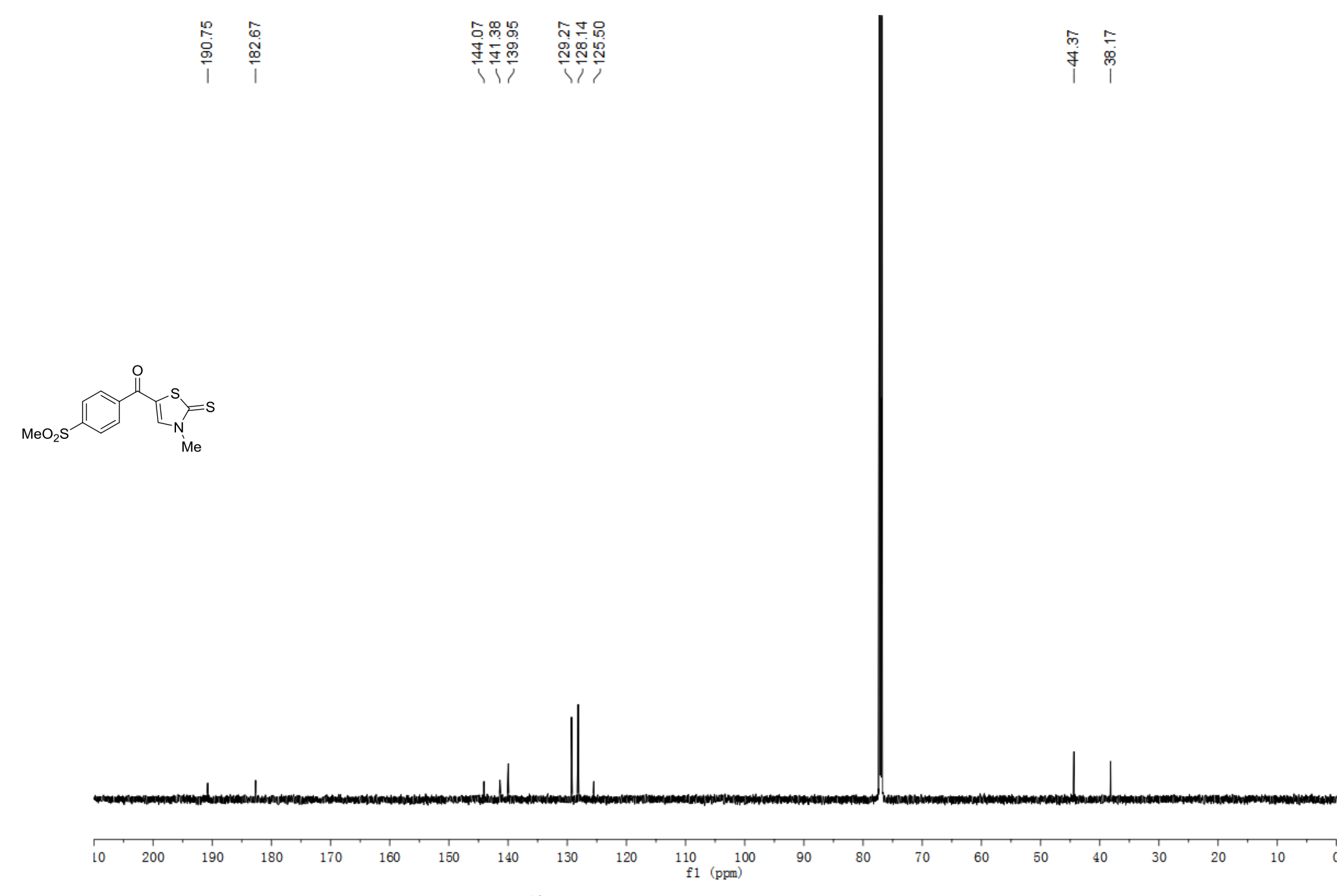

Figure S23. ${ }^{13} \mathrm{C}$ NMR $\left(150 \mathrm{MHz}, \mathrm{CDCl}_{3}\right)$ spectra of compound $\mathbf{2 k}$ 

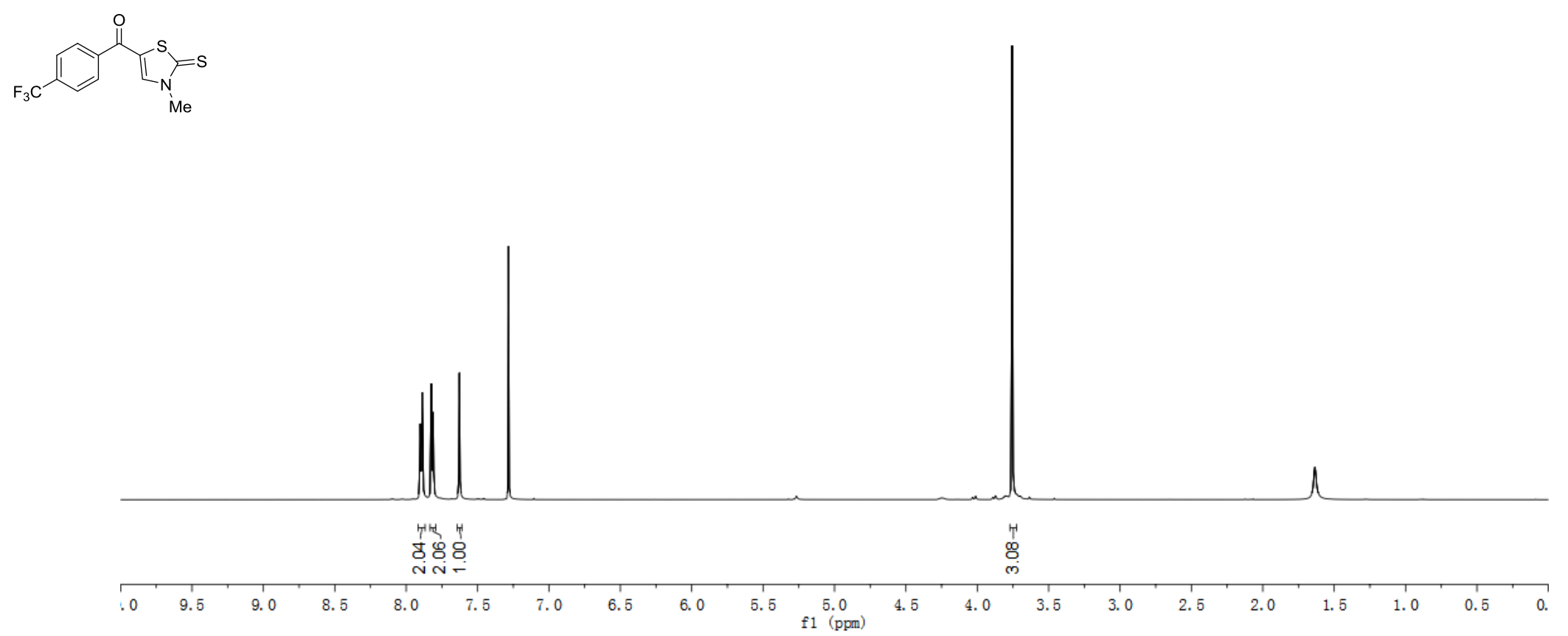

Figure S24. ${ }^{1} \mathrm{H}$ NMR $\left(600 \mathrm{MHz}, \mathrm{CDCl}_{3}\right)$ spectra of compound $2 \mathbf{I}$ 


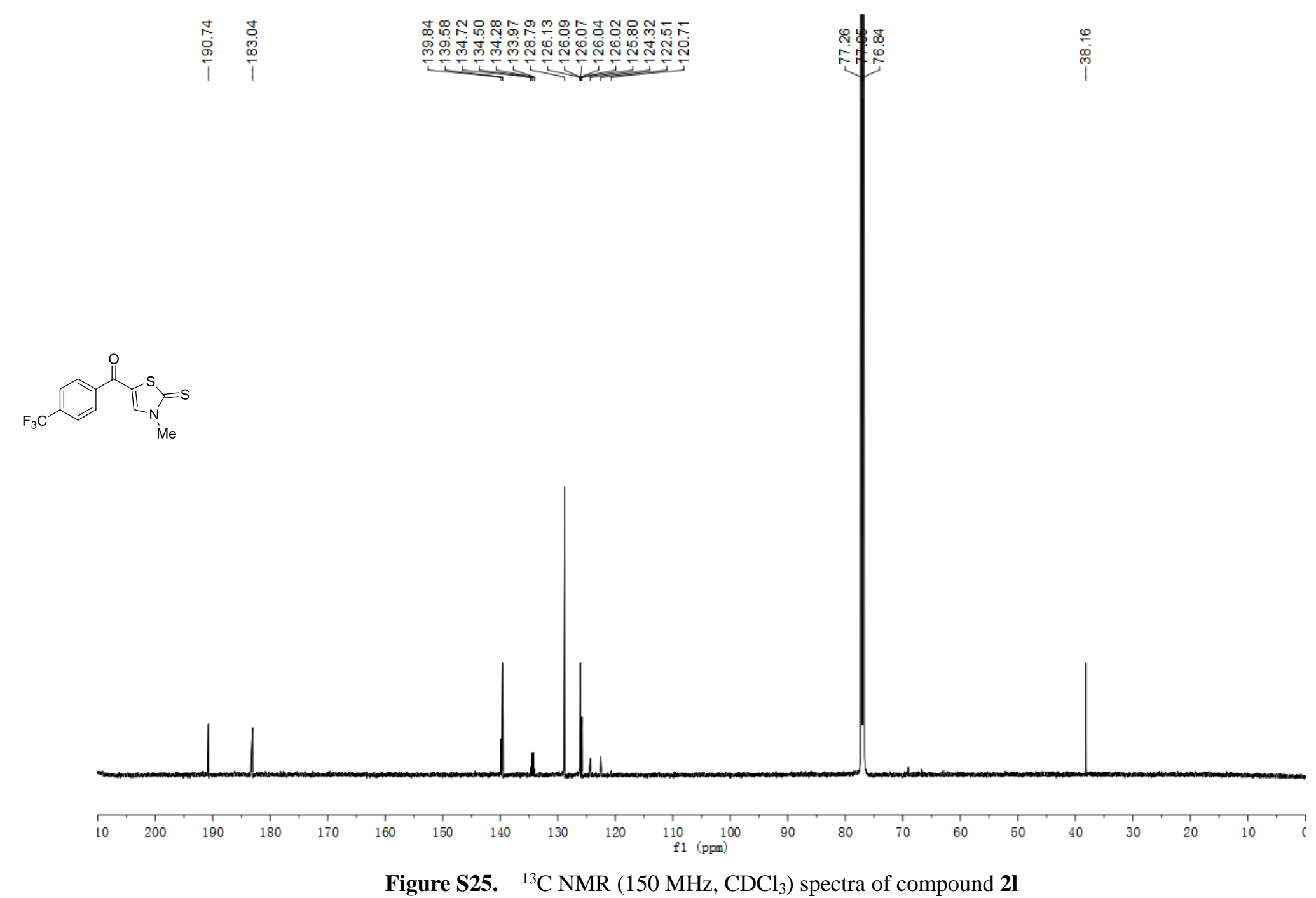




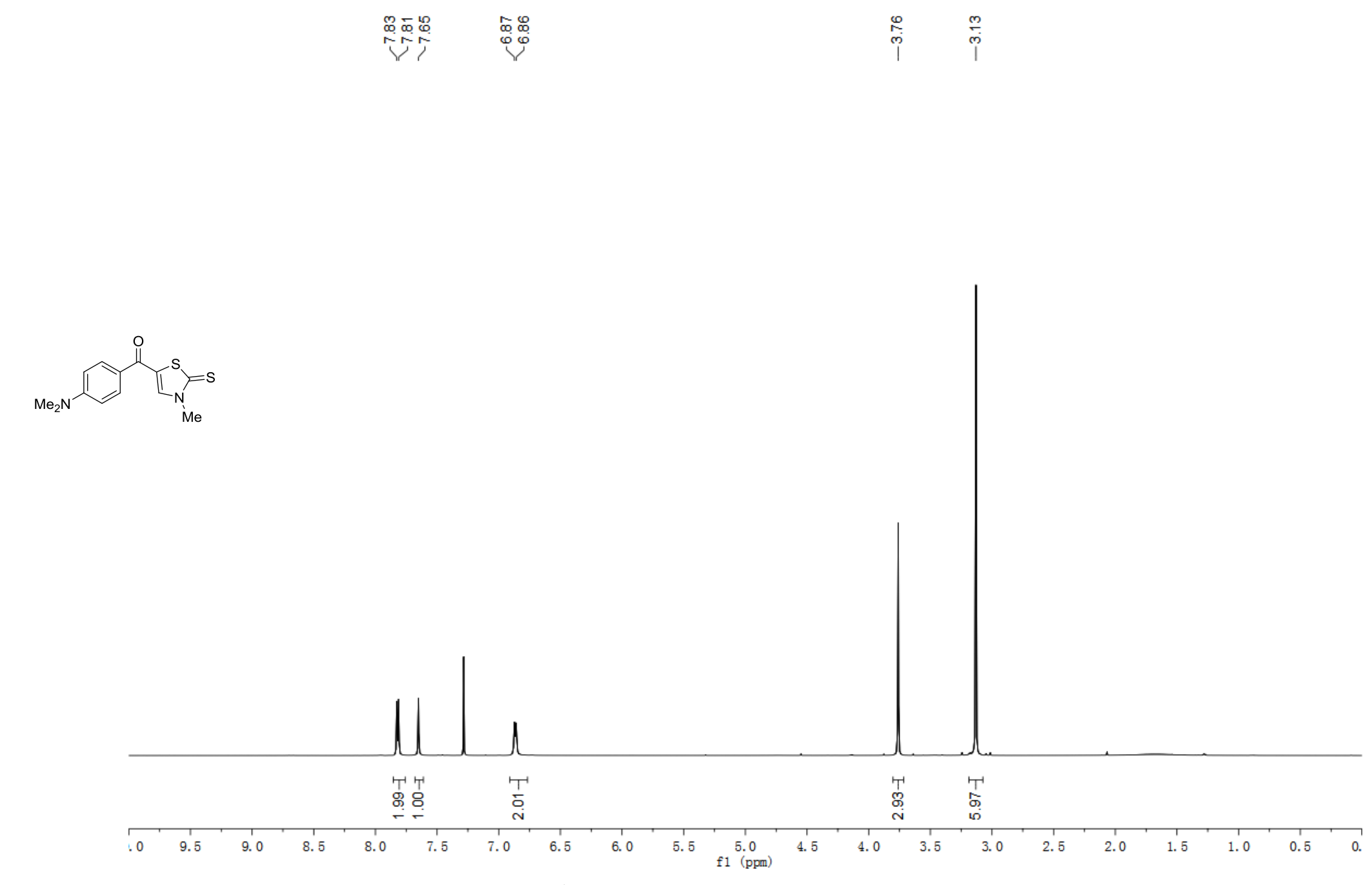

Figure S26. ${ }^{1} \mathrm{H}$ NMR $\left(600 \mathrm{MHz}, \mathrm{CDCl}_{3}\right)$ spectra of compound $\mathbf{2 m}$ 


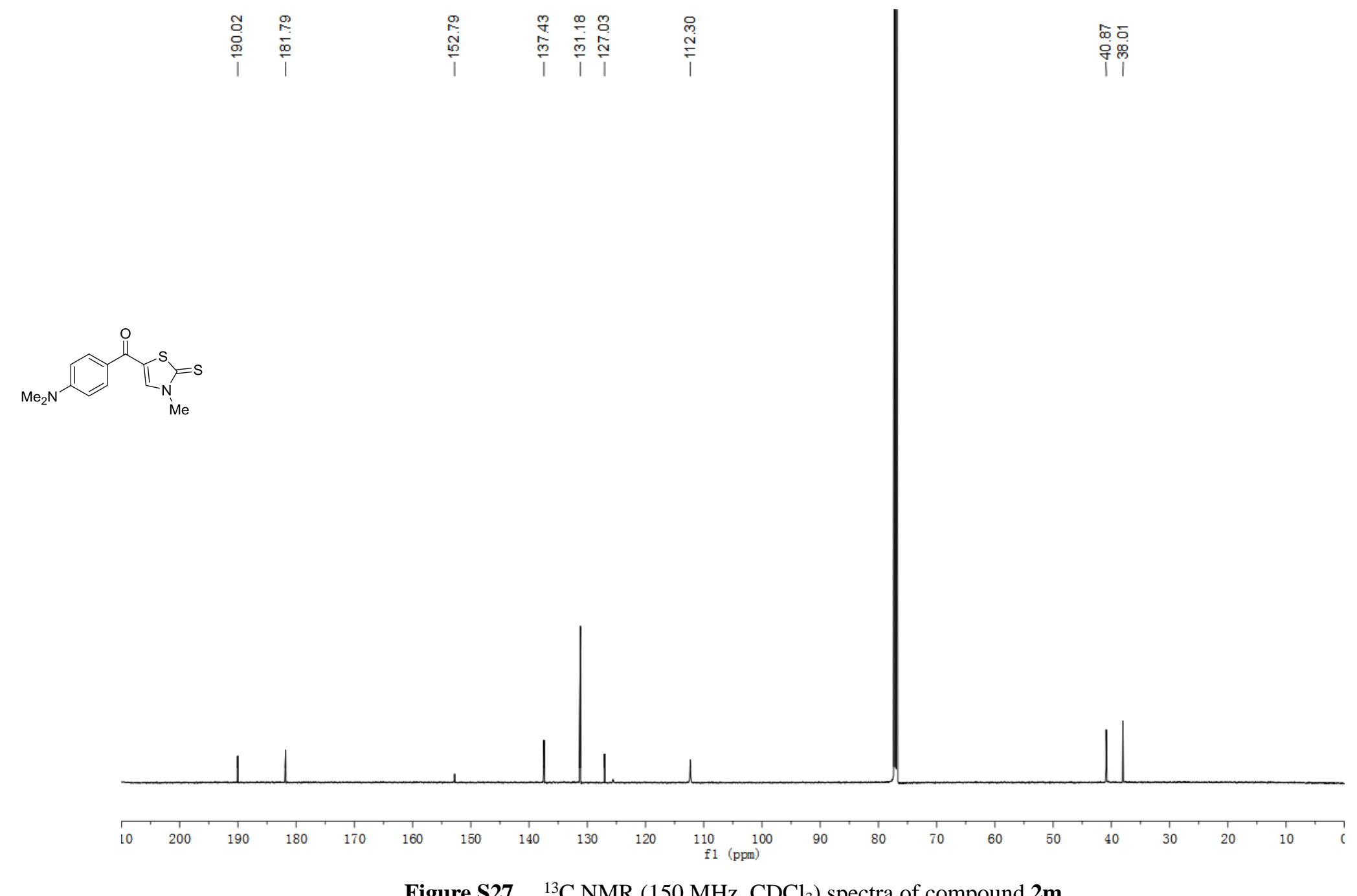

Figure S27. ${ }^{13} \mathrm{C} \mathrm{NMR}\left(150 \mathrm{MHz}, \mathrm{CDCl}_{3}\right)$ spectra of compound $\mathbf{2 m}$ 

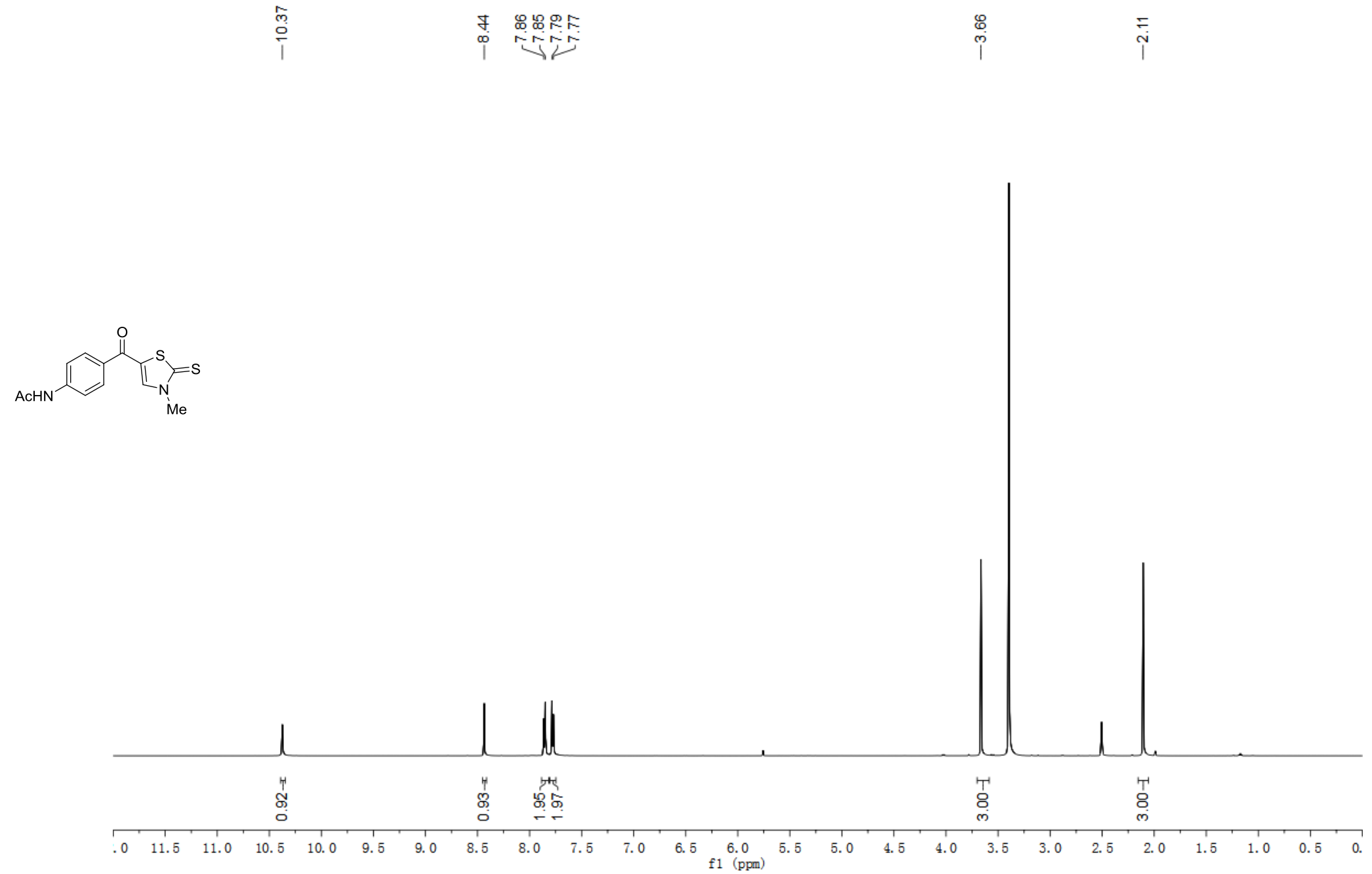

Figure S28. $\quad{ }^{1} \mathrm{H}$ NMR (600 MHz, DMSO- $\left.d_{6}\right)$ spectra of compound 2n 


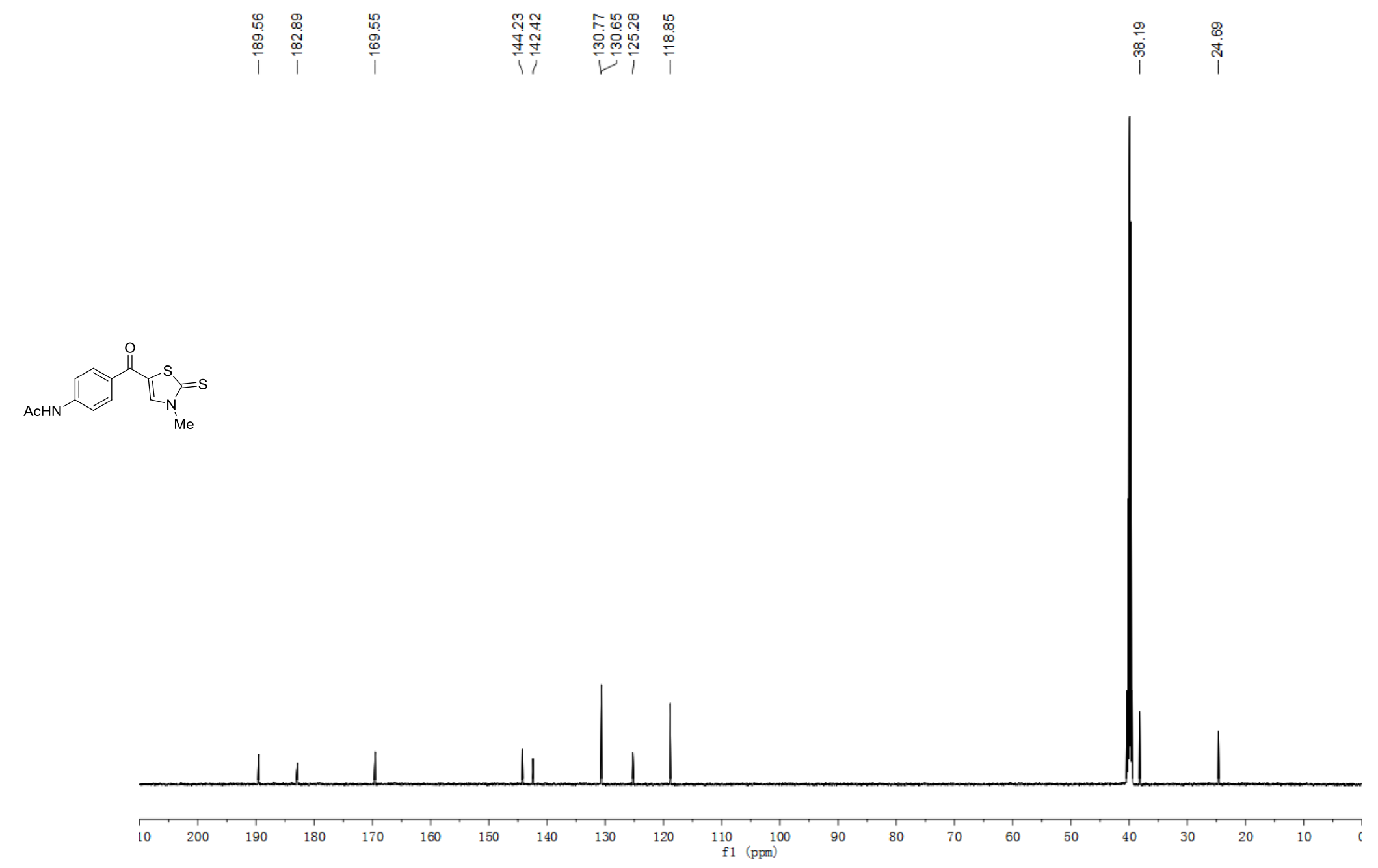

Figure S29. ${ }^{13} \mathrm{C}$ NMR $\left(150 \mathrm{MHz}, \mathrm{DMSO}-d_{6}\right)$ spectra of compound $\mathbf{2 n}$ 

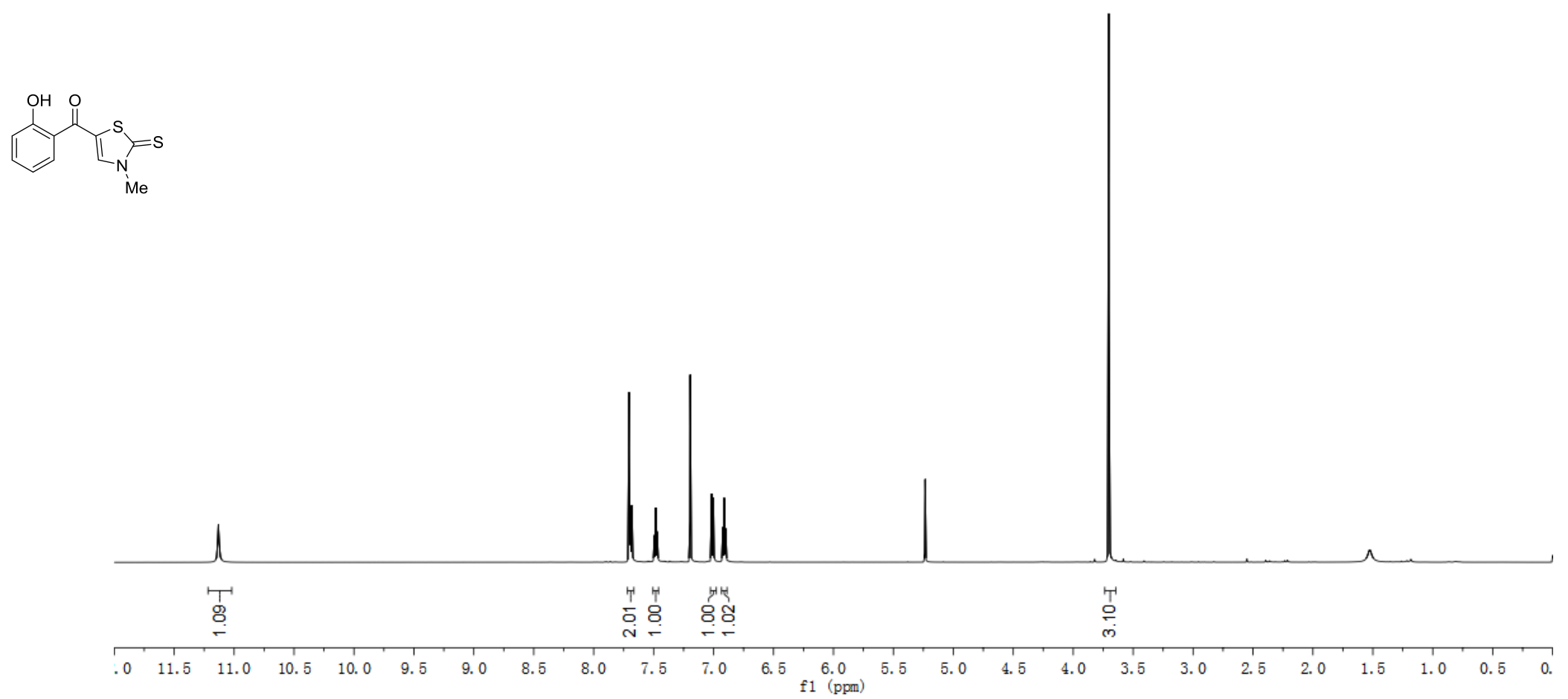

Figure S30. ${ }^{1} \mathrm{H}$ NMR $\left(600 \mathrm{MHz}, \mathrm{CDCl}_{3}\right)$ spectra of compound 20 


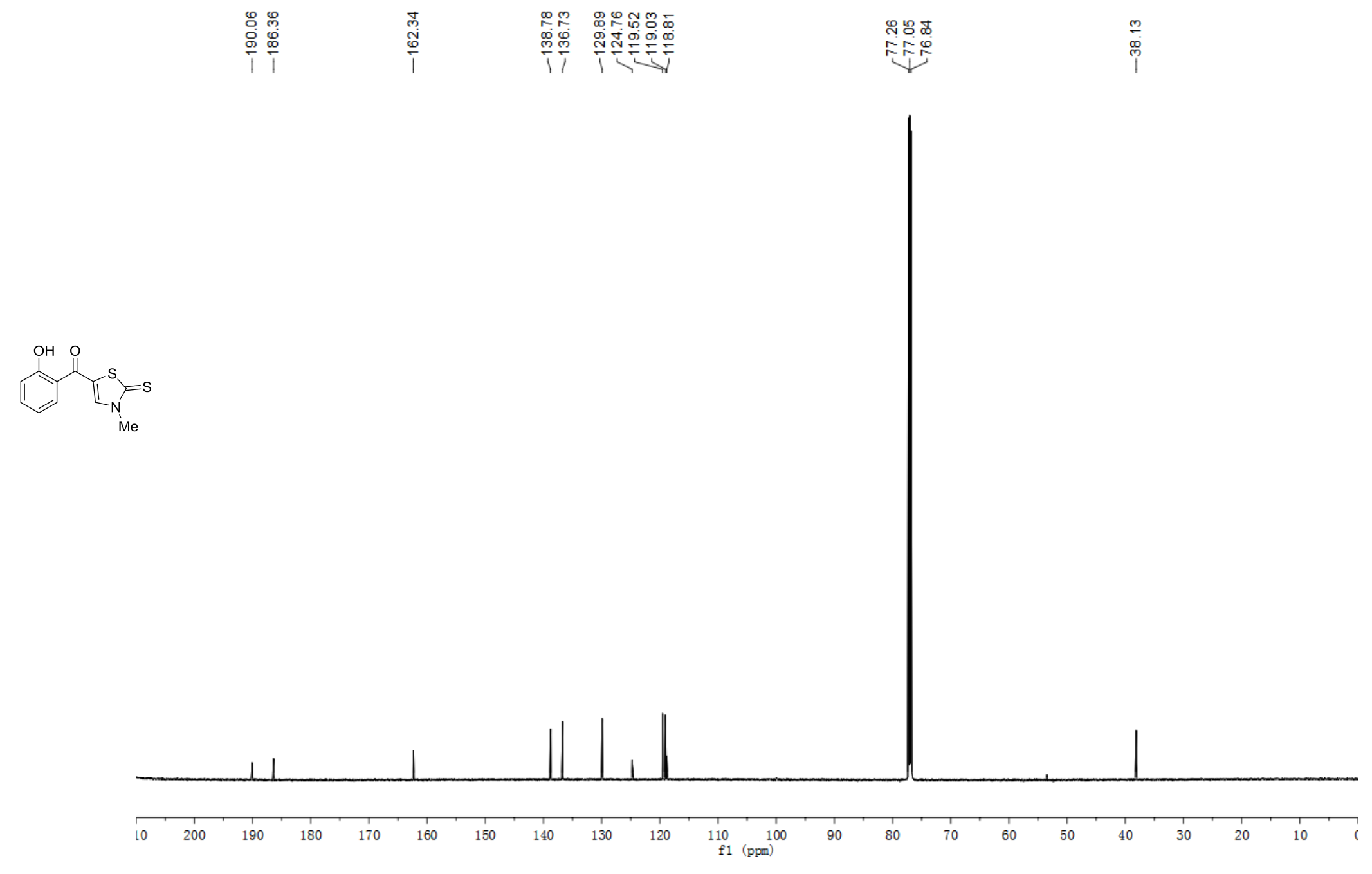

Figure S31. ${ }^{13} \mathrm{C}$ NMR $\left(150 \mathrm{MHz}, \mathrm{CDCl}_{3}\right)$ spectra of compound 20 

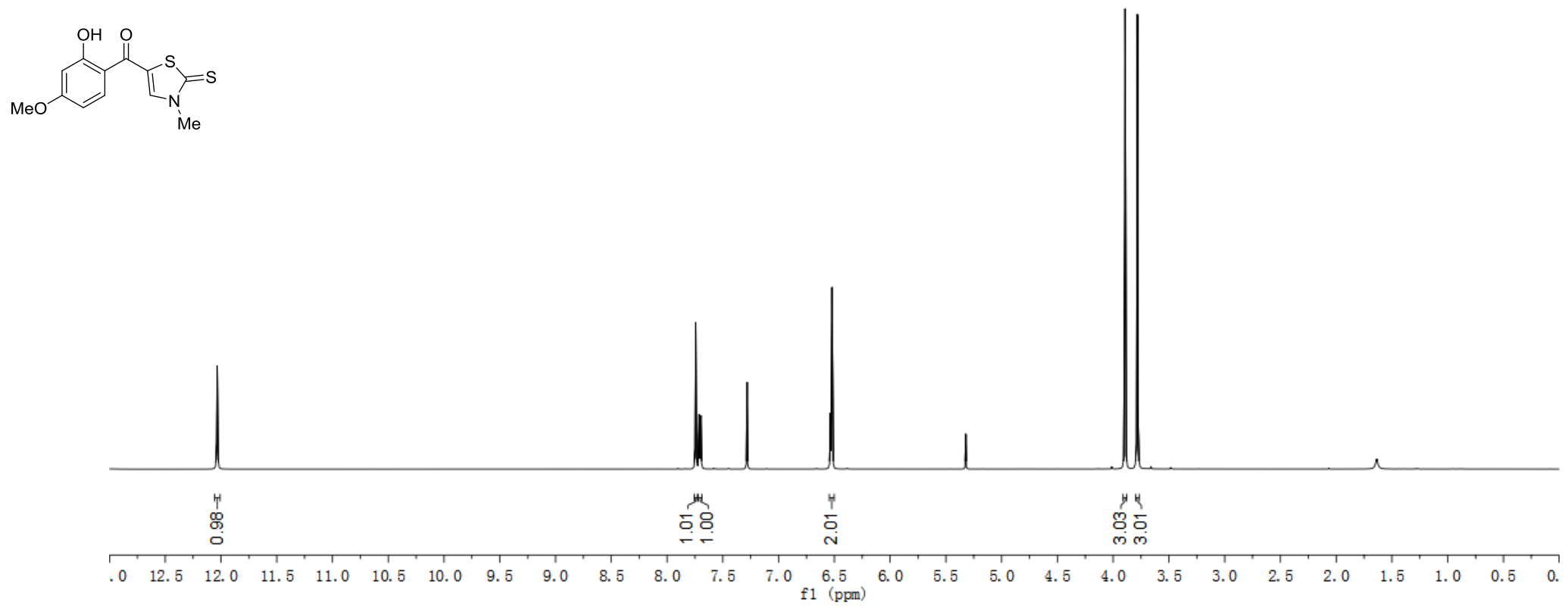

Figure S32. ${ }^{1} \mathrm{H}$ NMR (600 MHz, $\left.\mathrm{CDCl}_{3}\right)$ spectra of compound 2p 


\begin{tabular}{|c|c|c|c|c|c|c|c|c|}
\hline 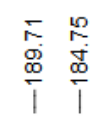 & 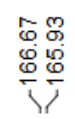 & $\underset{\substack{m \\
\stackrel{m}{m}}}{\stackrel{m}{i}}$ & $\frac{\stackrel{\leftrightarrow}{\oplus}}{\stackrel{m}{0}_{1}}$ & 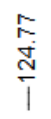 & 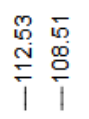 & 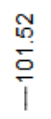 & 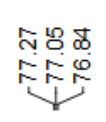 & مْ \\
\hline
\end{tabular}

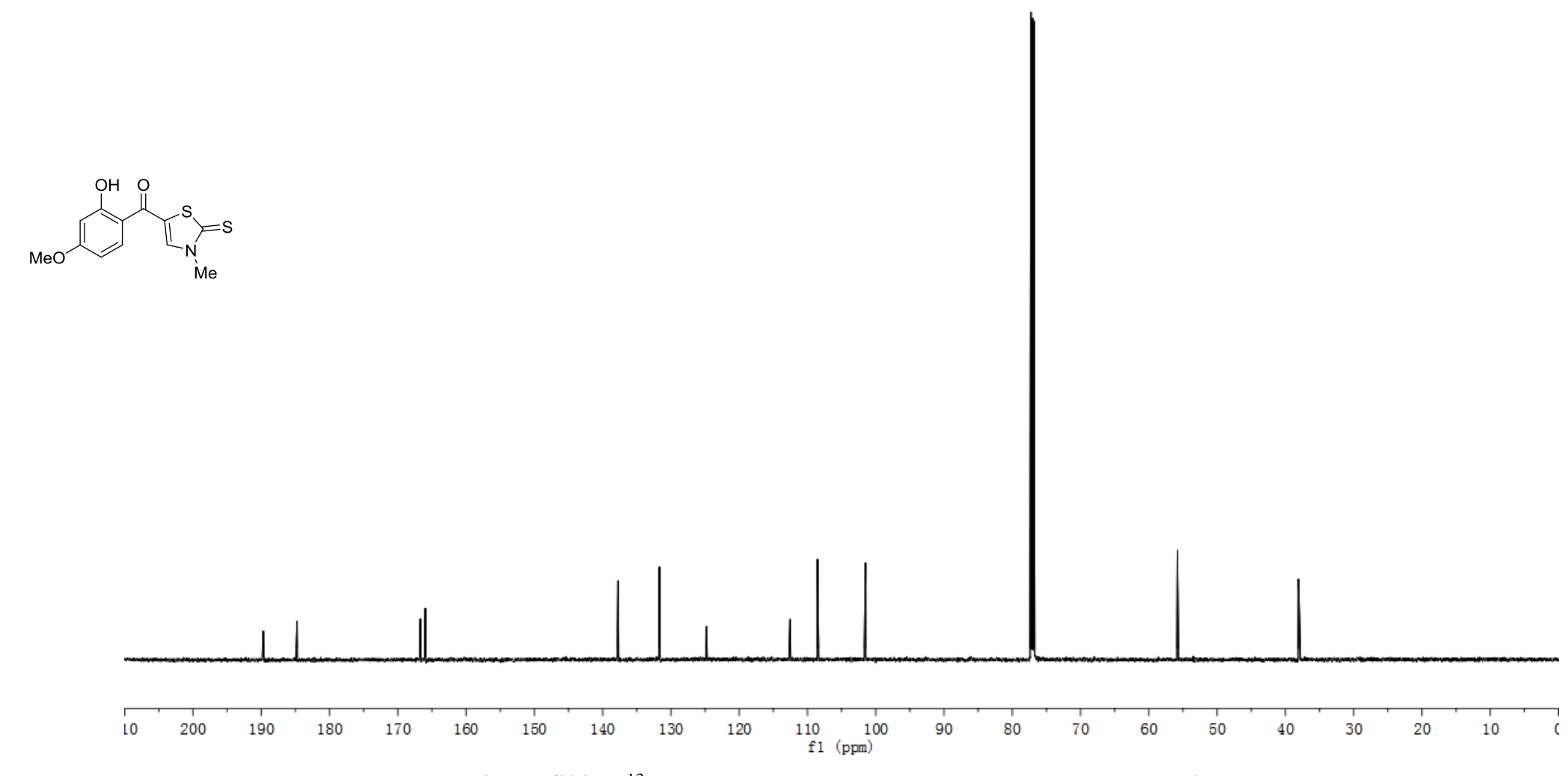

Figure S33. ${ }^{13} \mathrm{C} \mathrm{NMR}\left(150 \mathrm{MHz}, \mathrm{CDCl}_{3}\right)$ spectra of compound 2p 


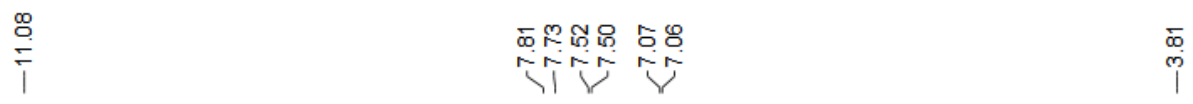

$\int_{n=s}^{s}$

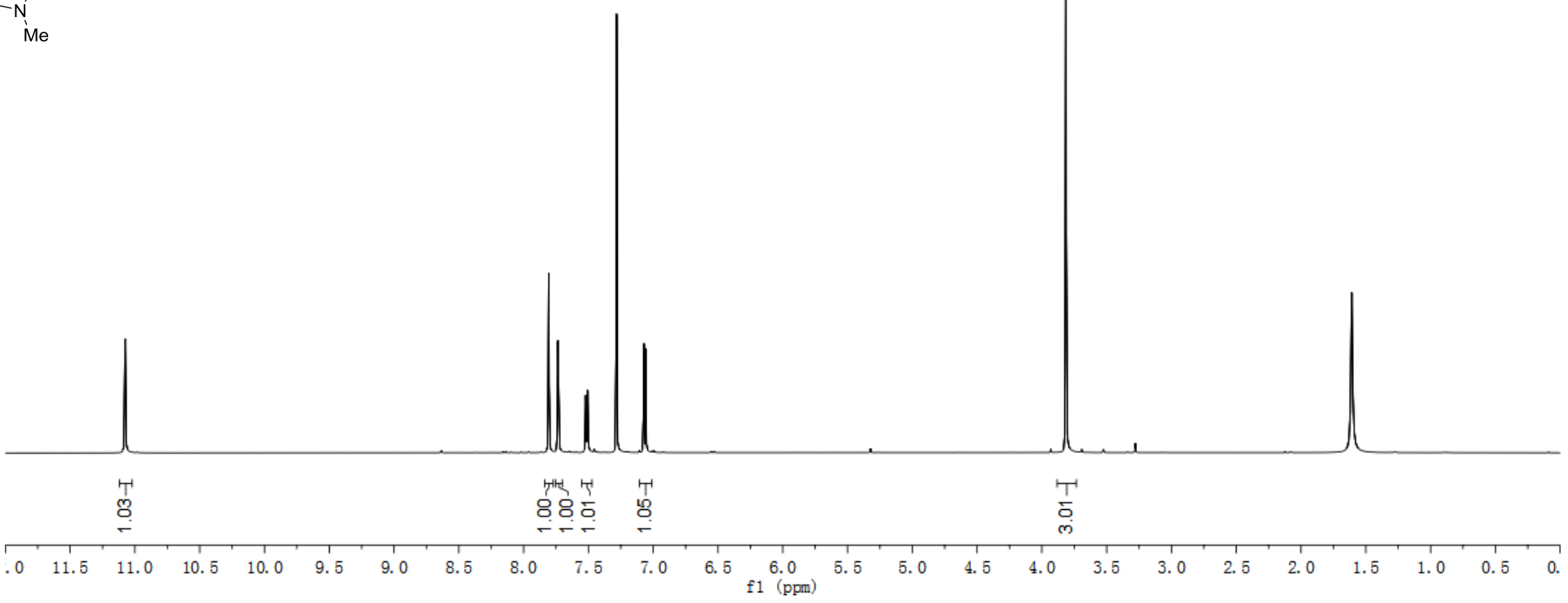

Figure S34. $\quad{ }^{1} \mathrm{H}$ NMR $\left(600 \mathrm{MHz}, \mathrm{CDCl}_{3}\right)$ spectra of compound $\mathbf{2 q}$ 


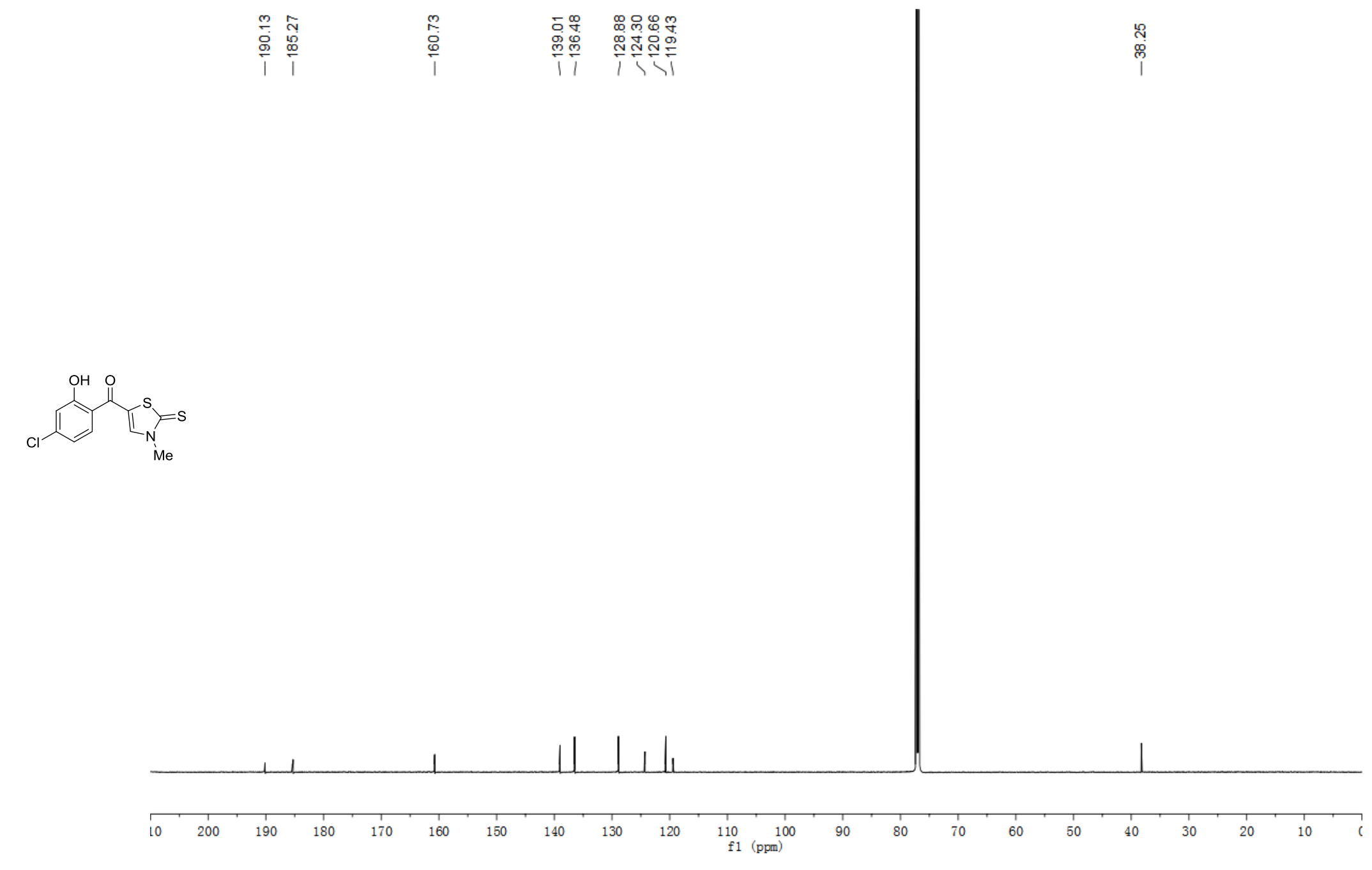

Figure S35. ${ }^{13} \mathrm{C}$ NMR $\left(150 \mathrm{MHz}, \mathrm{CDCl}_{3}\right)$ spectra of compound $\mathbf{2 q}$ 


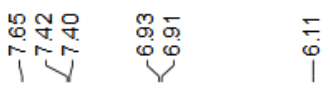

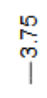
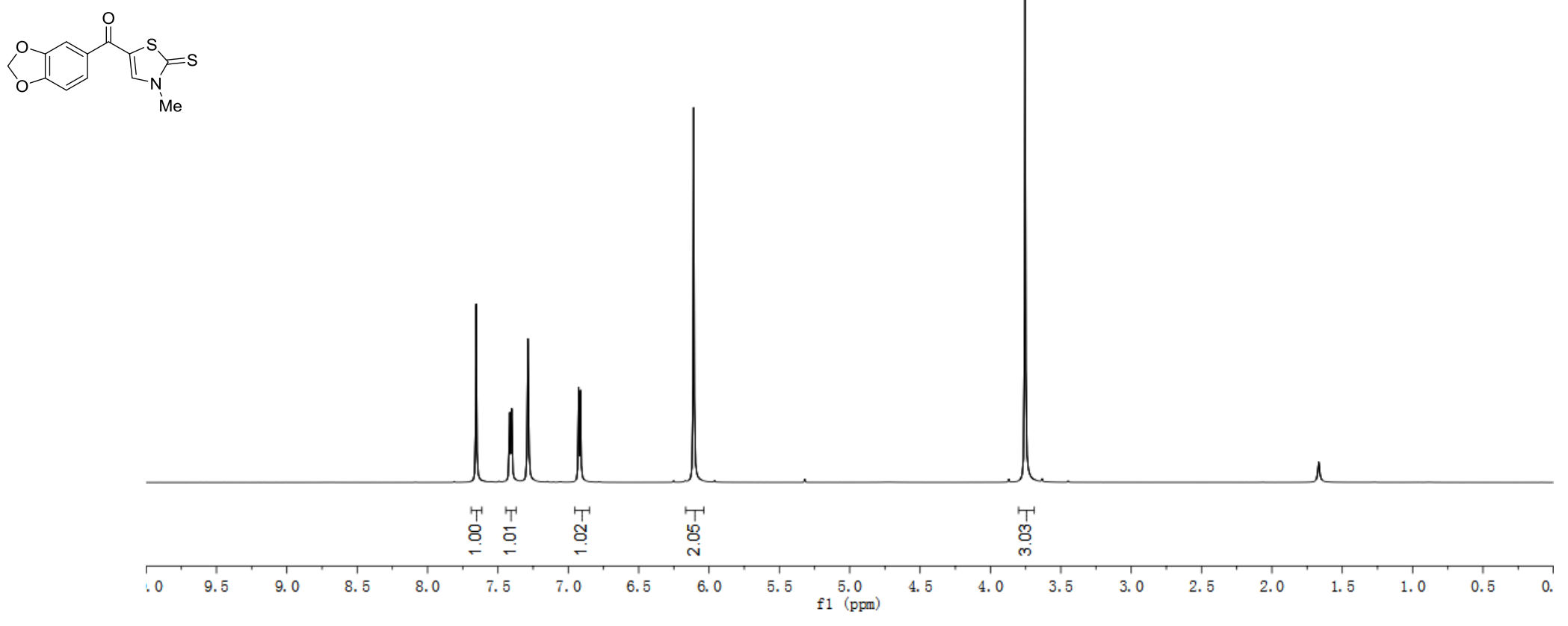

Figure S36. $\quad{ }^{1} \mathrm{H} \mathrm{NMR}\left(600 \mathrm{MHz}, \mathrm{CDCl}_{3}\right)$ spectra of compound $\mathbf{2 r}$

S56 


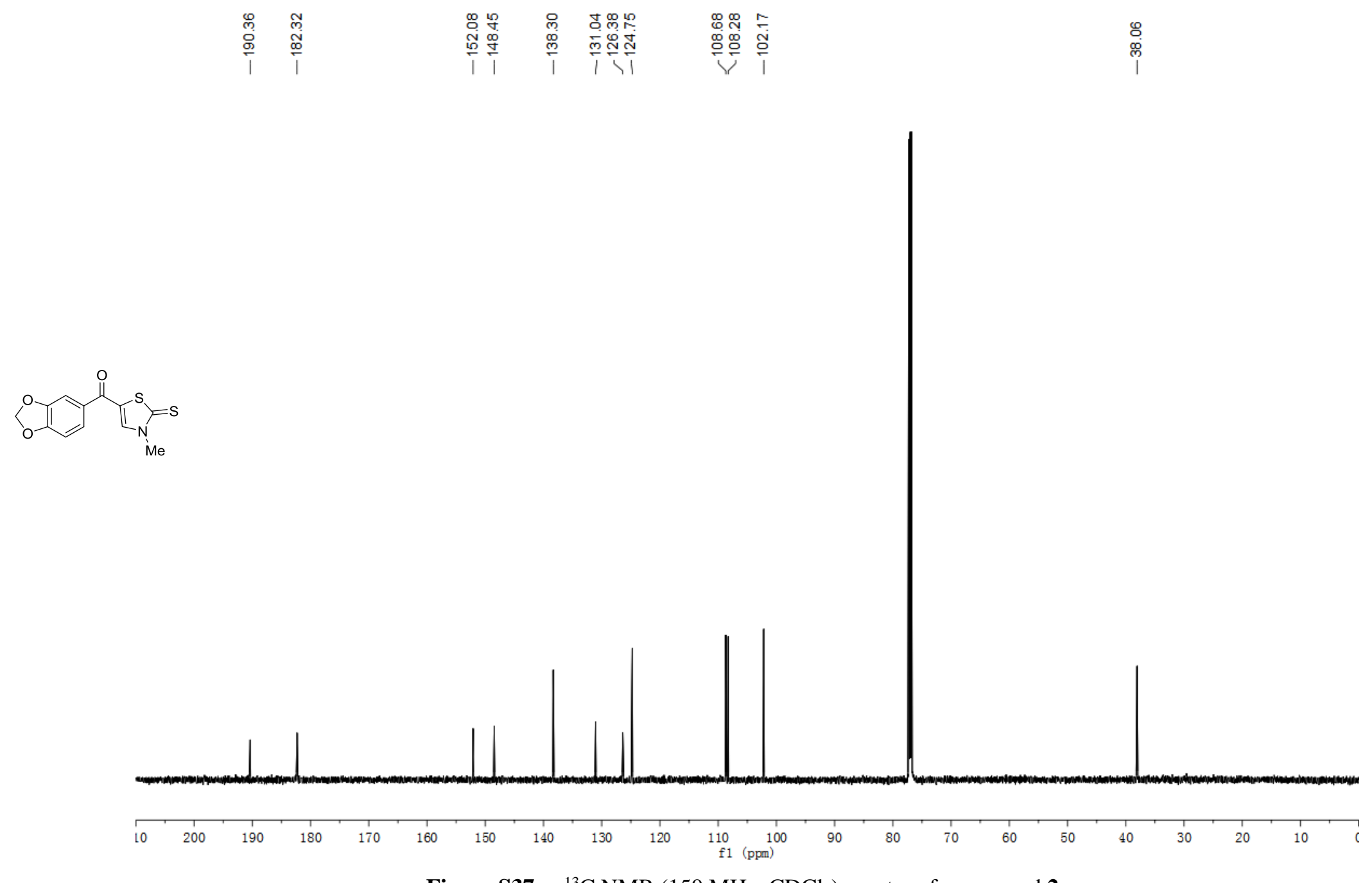

Figure S37. ${ }^{13} \mathrm{C}$ NMR $\left(150 \mathrm{MHz}, \mathrm{CDCl}_{3}\right)$ spectra of compound $\mathbf{2 r}$ 

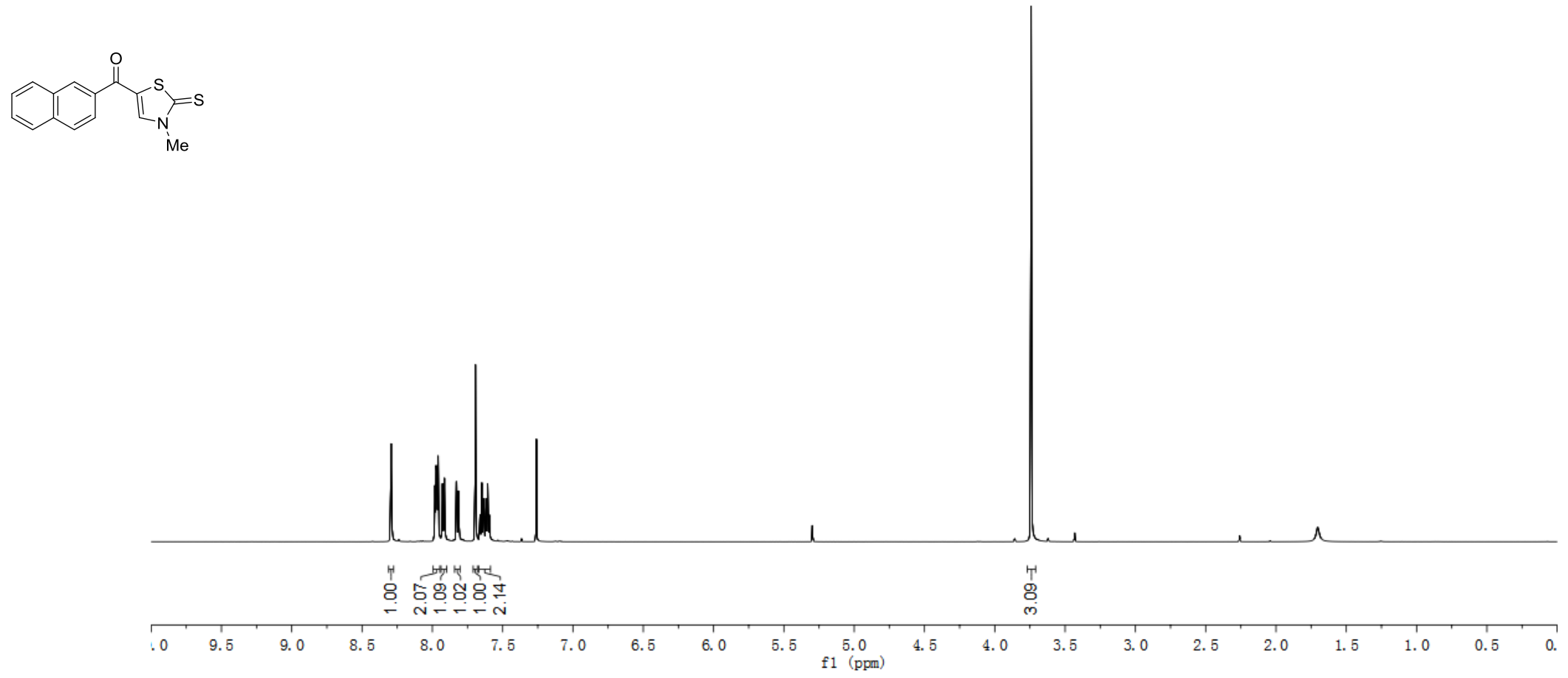

Figure S38. $\quad{ }^{1} \mathrm{H}$ NMR $\left(600 \mathrm{MHz}, \mathrm{CDCl}_{3}\right)$ spectra of compound 2s 

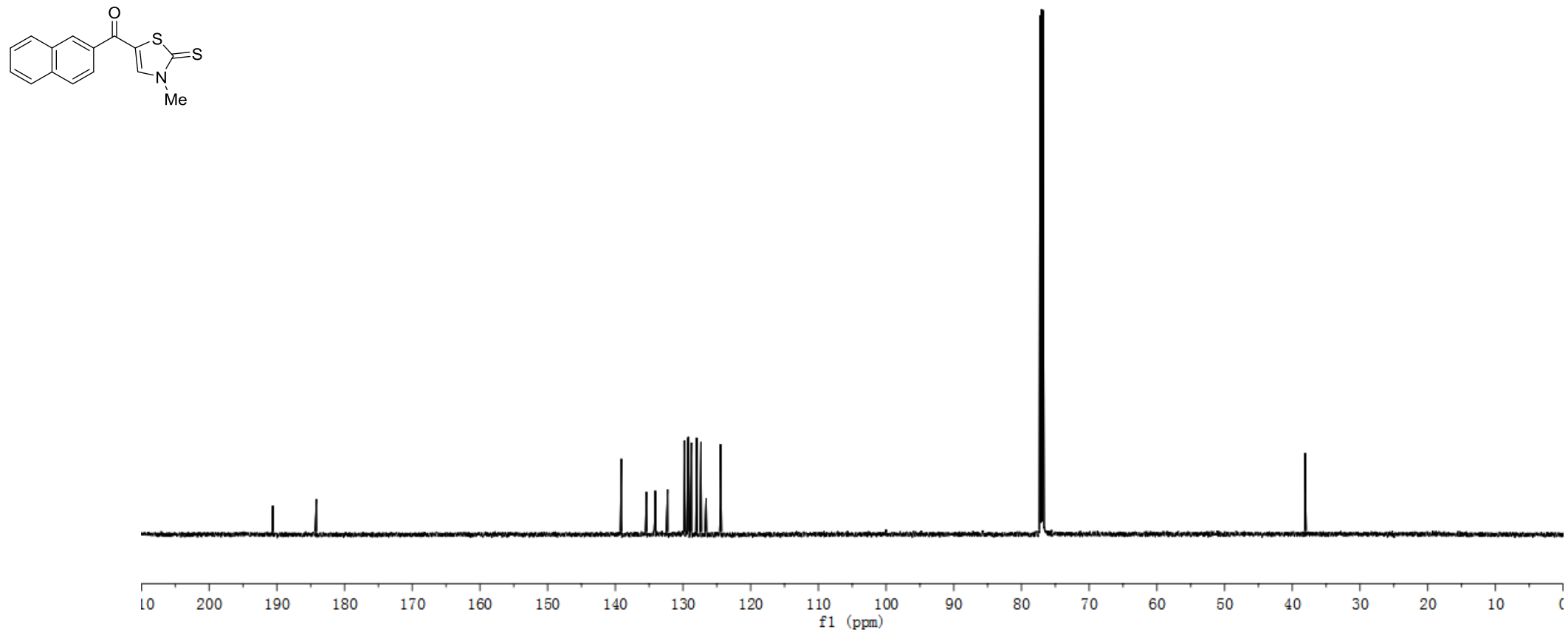

Figure S39. ${ }^{13} \mathrm{C} \mathrm{NMR}\left(150 \mathrm{MHz}, \mathrm{CDCl}_{3}\right)$ spectra of compound $2 \mathrm{~s}$ 


$$
1
$$




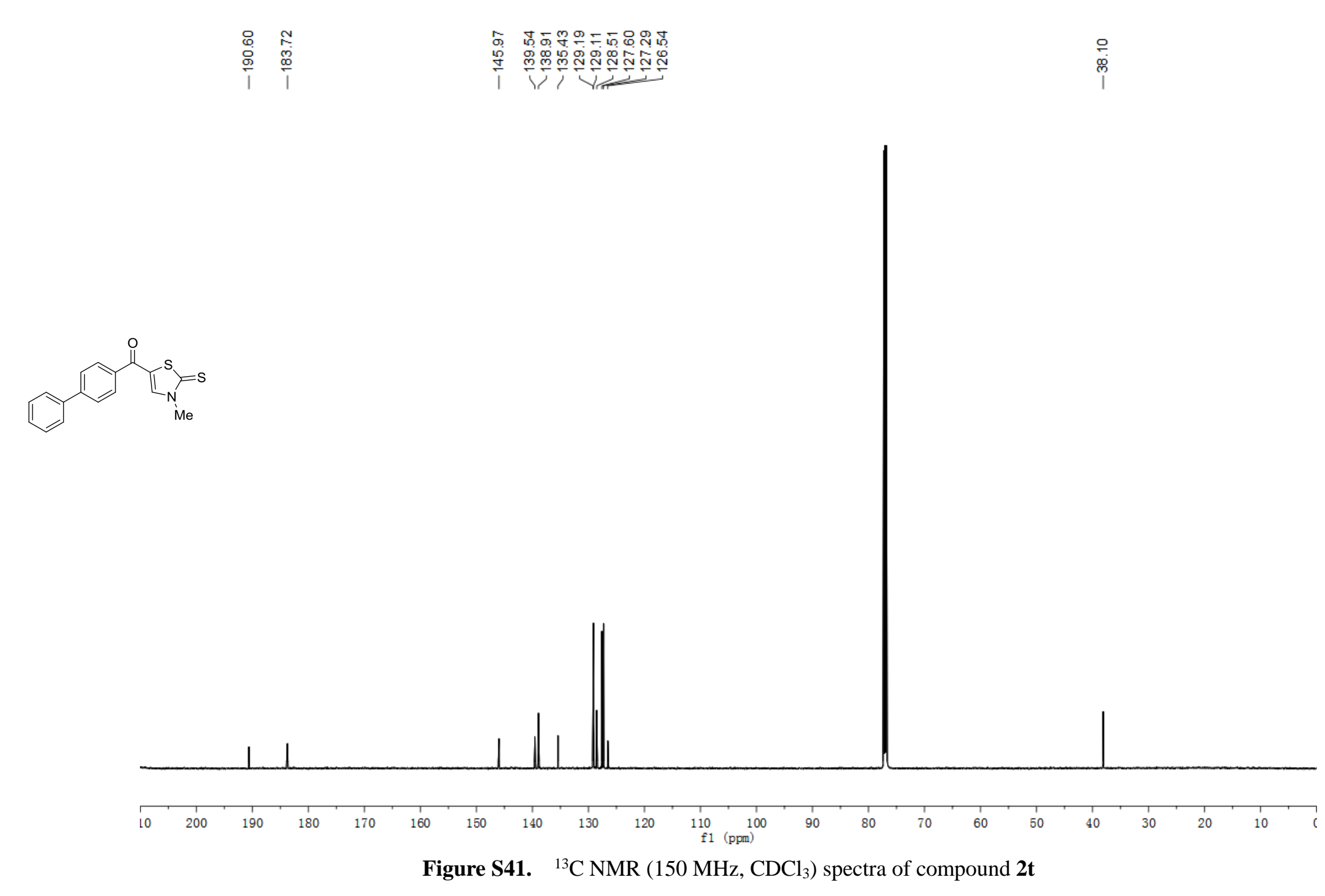




$$
4
$$




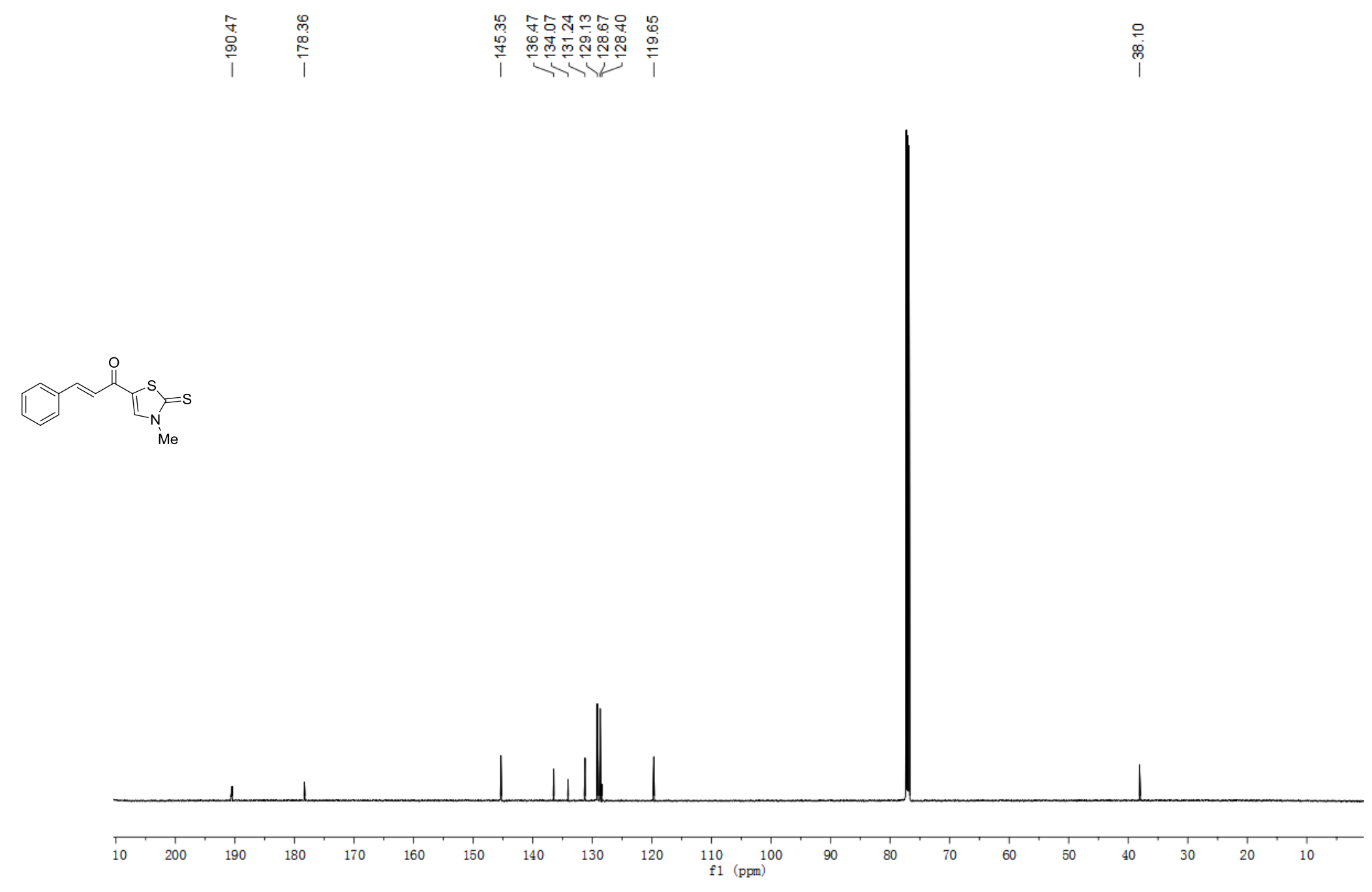

Figure S43. ${ }^{13} \mathrm{C}$ NMR $\left(150 \mathrm{MHz}, \mathrm{CDCl}_{3}\right)$ spectra of compound $\mathbf{2 u}$ 
$\underbrace{\infty}$

$\stackrel{\substack{0 \\ i}}{i}$
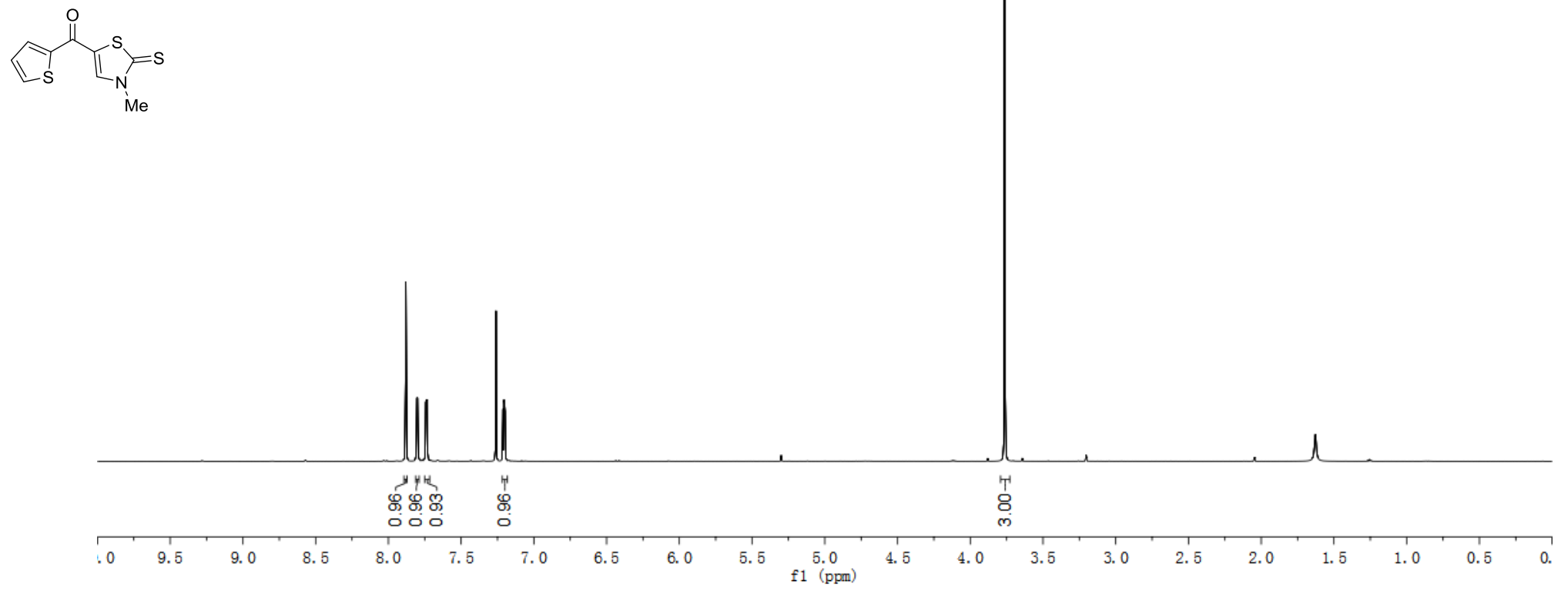

Figure S44. $\quad{ }^{1} \mathrm{H}$ NMR $\left(600 \mathrm{MHz}, \mathrm{CDCl}_{3}\right)$ spectra of compound $\mathbf{2 v}$

S64 


$$
=1
$$



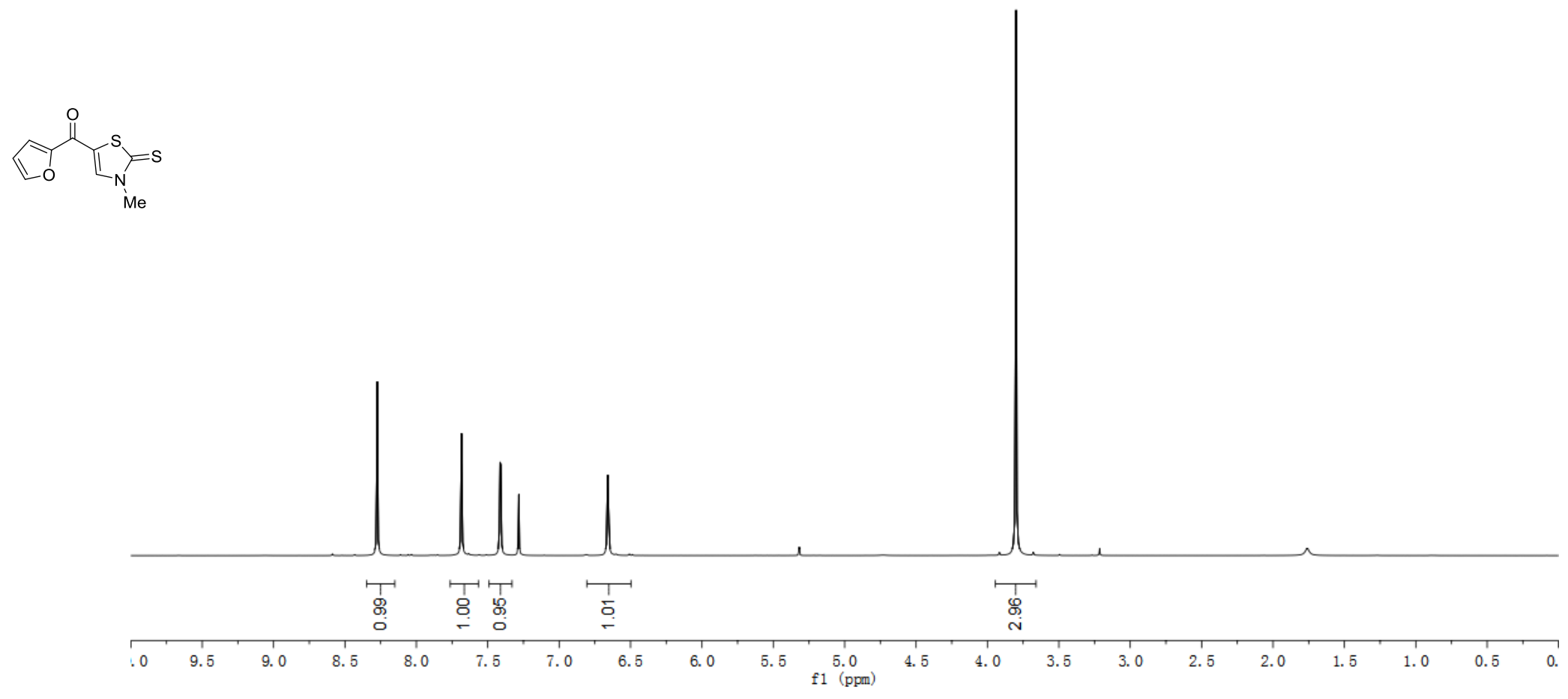

Figure S46. ${ }^{1} \mathrm{H}$ NMR (600 MHz, $\left.\mathrm{CDCl}_{3}\right)$ spectra of compound $\mathbf{2 w}$ 


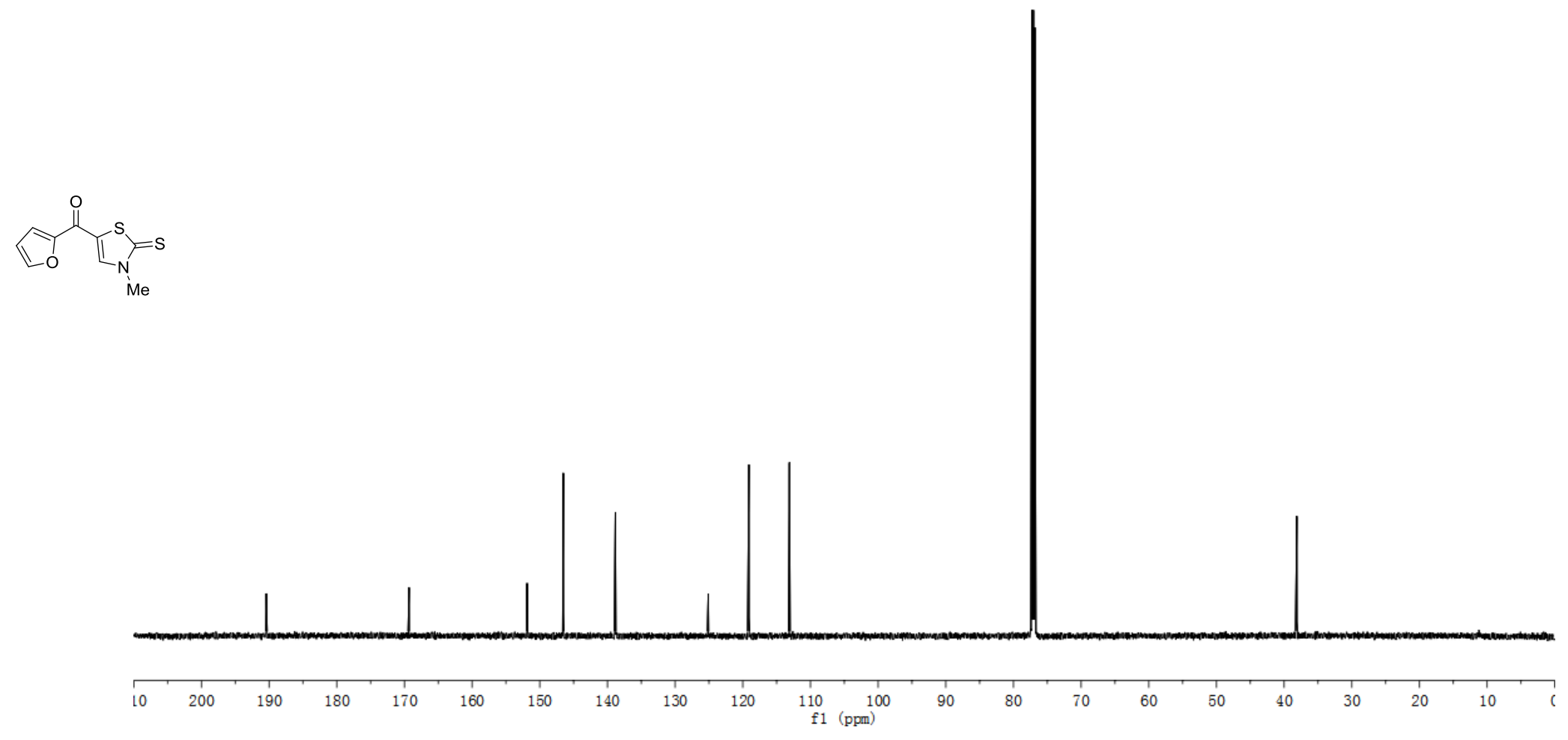

Figure S47. ${ }^{13} \mathrm{C} \mathrm{NMR}\left(150 \mathrm{MHz}, \mathrm{CDCl}_{3}\right)$ spectra of compound $\mathbf{2 w}$ 

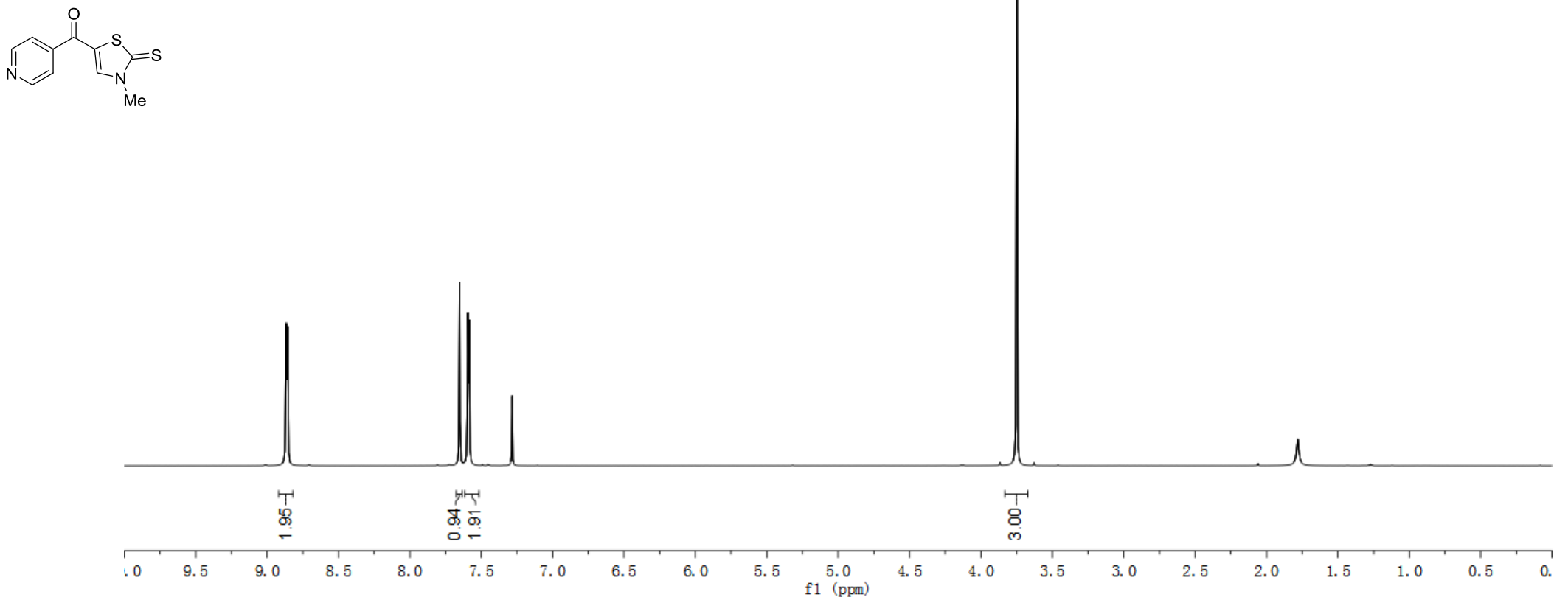

Figure S48. $\quad{ }^{1} \mathrm{H}$ NMR $\left(600 \mathrm{MHz}, \mathrm{CDCl}_{3}\right)$ spectra of compound $\mathbf{2 x}$ 


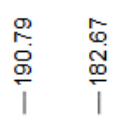

군

1

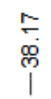
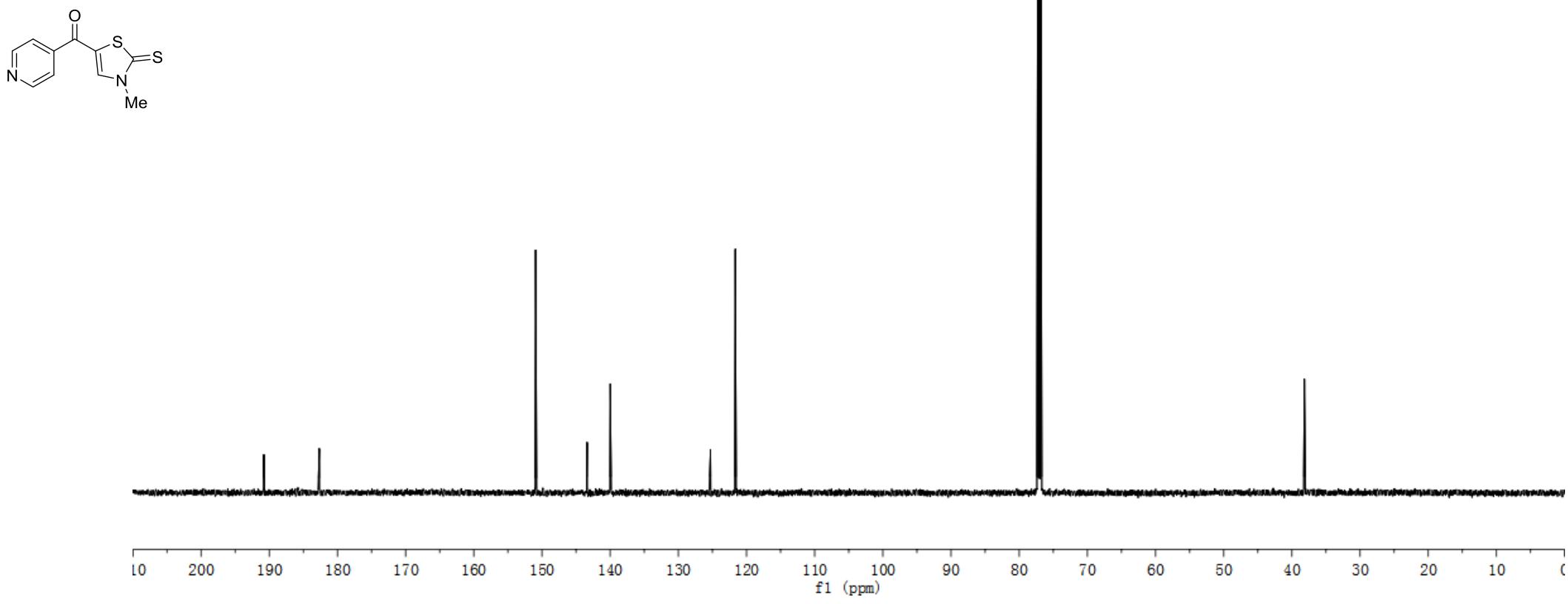

Figure S49. ${ }^{13} \mathrm{C}$ NMR ( $\left.150 \mathrm{MHz}, \mathrm{CDCl}_{3}\right)$ spectra of compound $\mathbf{2 x}$

S69 

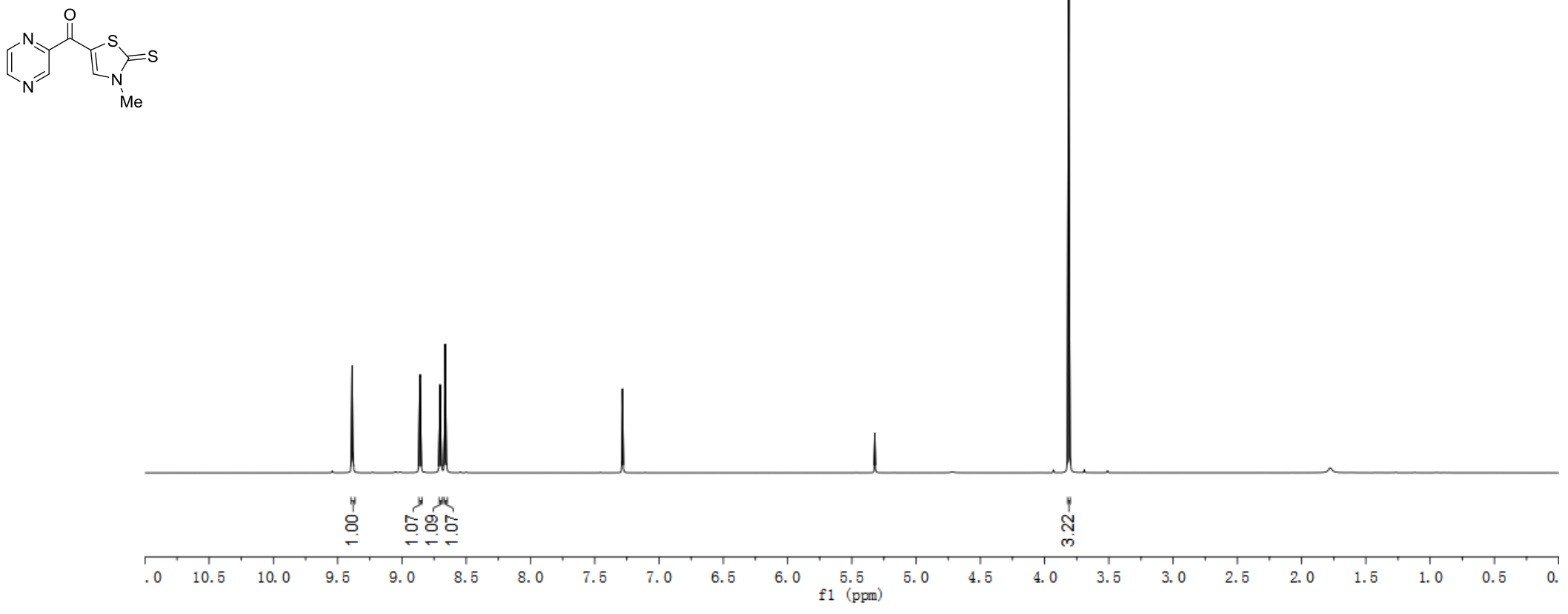

Figure S50. $\quad{ }^{1} \mathrm{H} \mathrm{NMR}\left(600 \mathrm{MHz}, \mathrm{CDCl}_{3}\right)$ spectra of compound $\mathbf{2 y}$ 
$\frac{8}{\bar{\sigma}} \quad \frac{0}{i}$

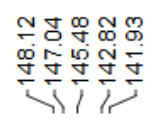

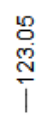

年

$\underset{\substack{\infty \\ \infty}}{\substack{\infty \\ 1}}$
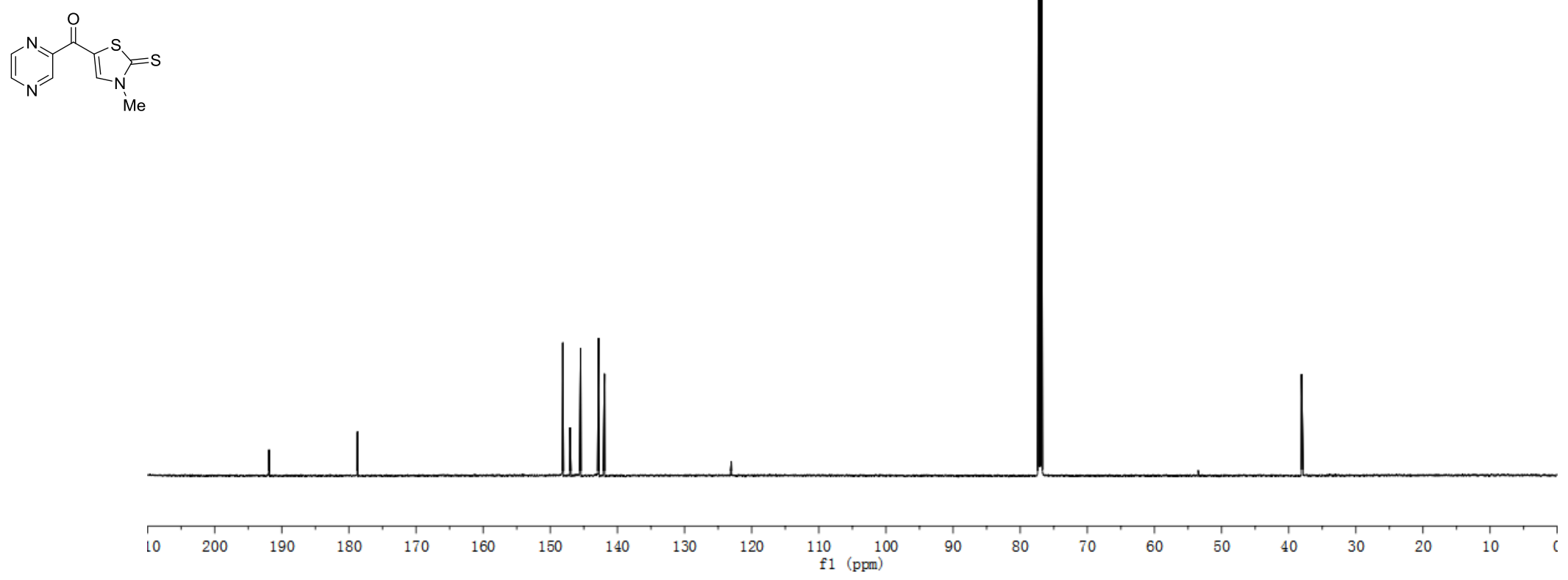

Figure S51. ${ }^{13} \mathrm{C}$ NMR $\left(150 \mathrm{MHz}, \mathrm{CDCl}_{3}\right)$ spectra of compound $\mathbf{2 y}$

S71 


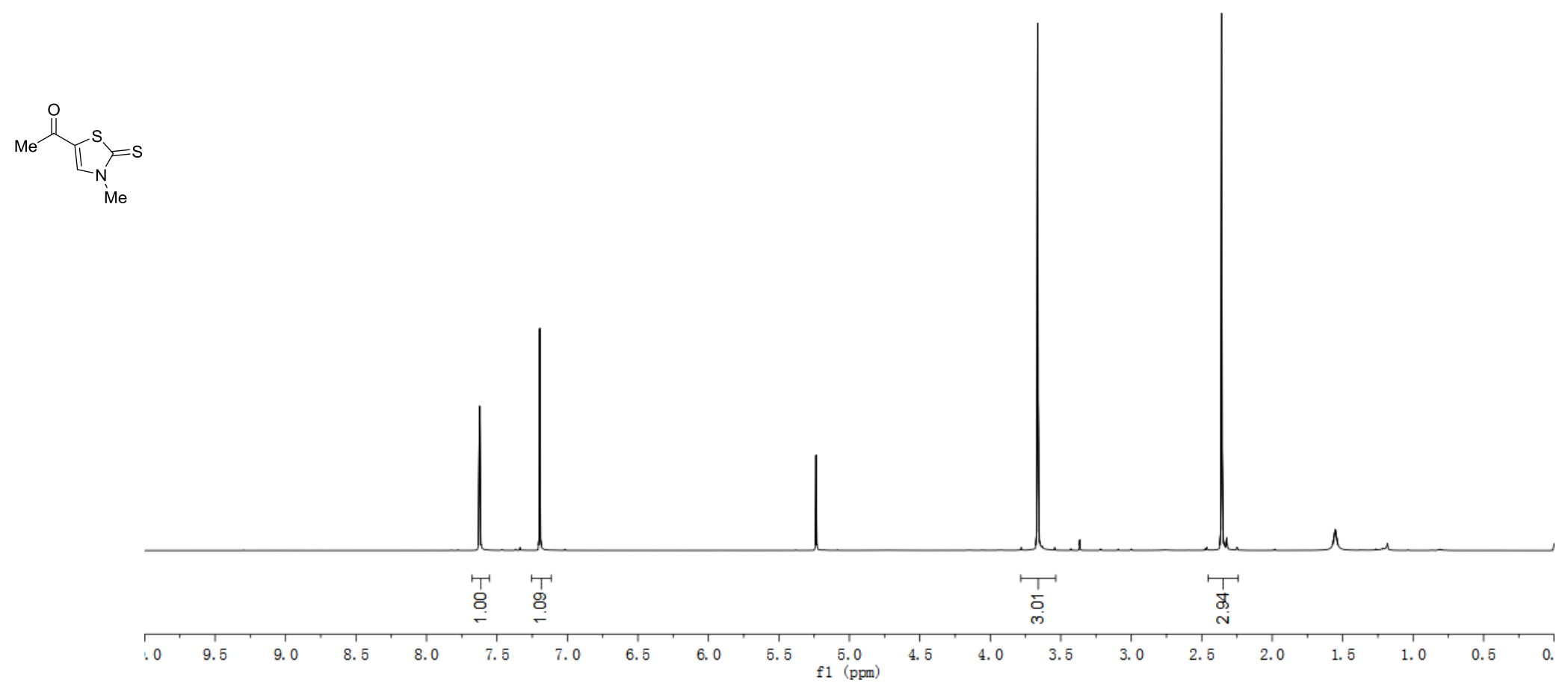

Figure S52. ${ }^{1} \mathrm{H}$ NMR $\left(600 \mathrm{MHz}, \mathrm{CDCl}_{3}\right)$ spectra of compound $\mathbf{2 z}$ 


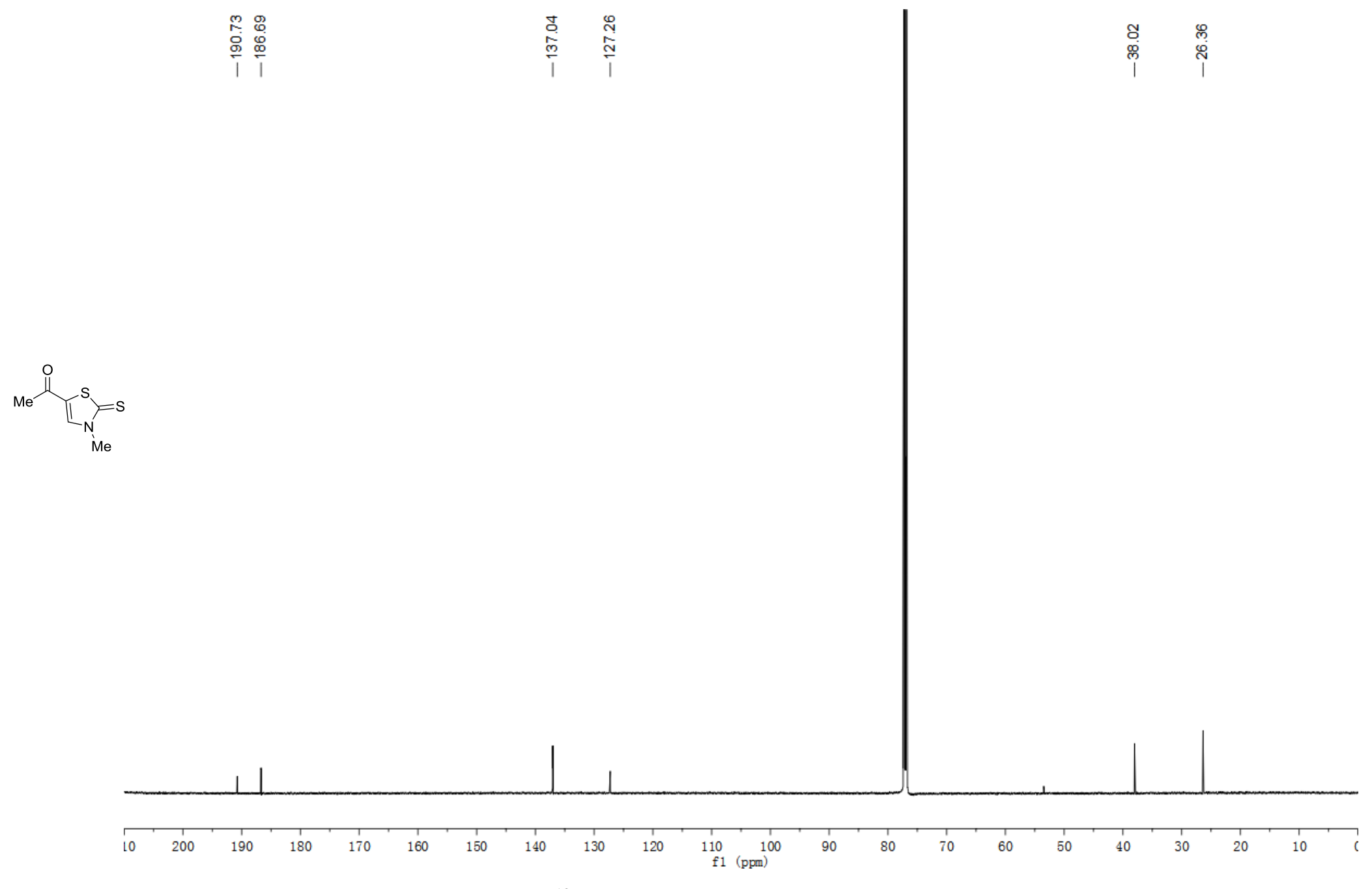

Figure S53. ${ }^{13} \mathrm{C}$ NMR $\left(150 \mathrm{MHz}, \mathrm{CDCl}_{3}\right)$ spectra of compound $\mathbf{2 z}$ 


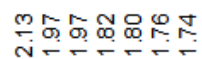
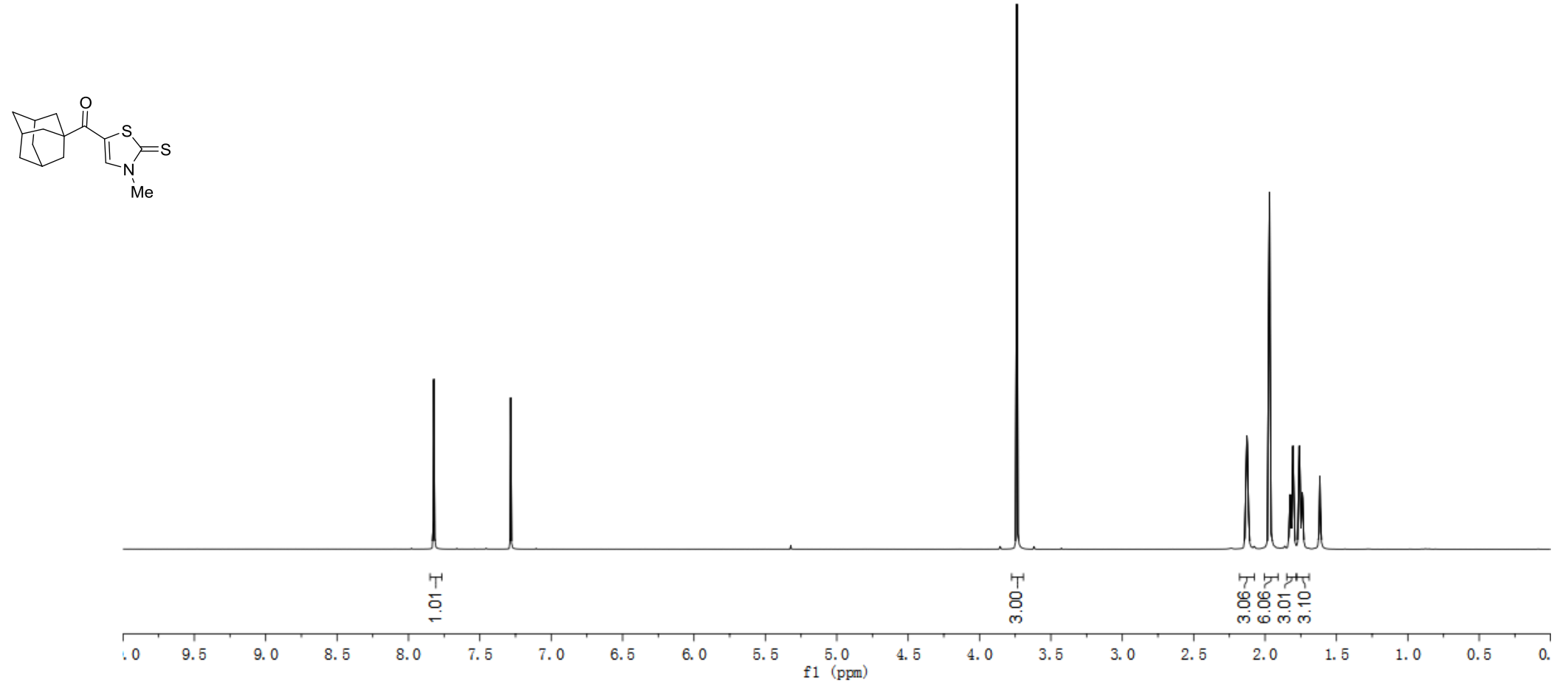

Figure S54. $\quad{ }^{1} \mathrm{H}$ NMR $\left(600 \mathrm{MHz}, \mathrm{CDCl}_{3}\right)$ spectra of compound 2a' 

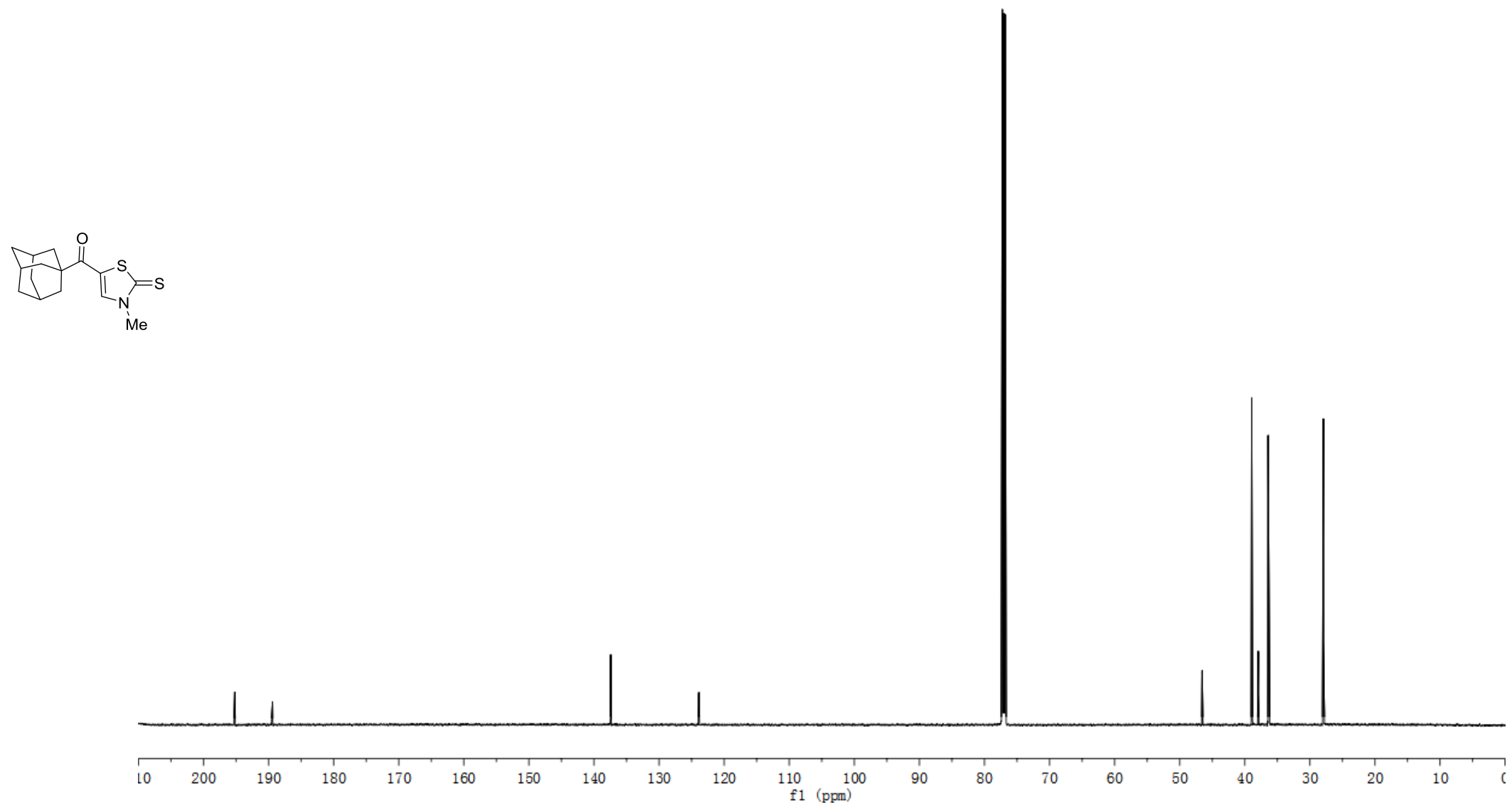

Figure S55. ${ }^{13} \mathrm{C} \mathrm{NMR}\left(150 \mathrm{MHz}, \mathrm{CDCl}_{3}\right)$ spectra of compound 2a' 

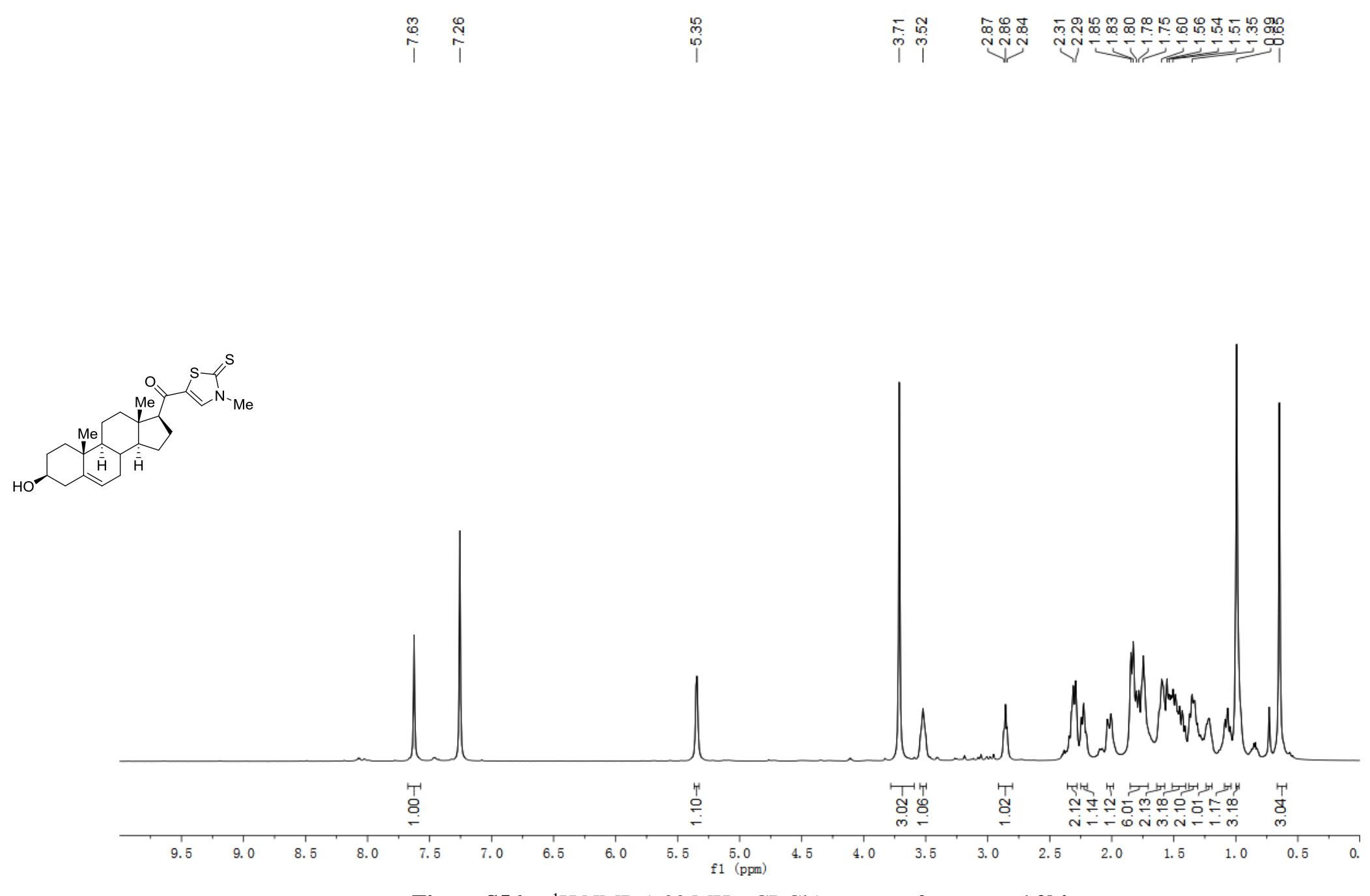

Figure S56. ${ }^{1} \mathrm{H} \mathrm{NMR}\left(600 \mathrm{MHz}, \mathrm{CDCl}_{3}\right)$ spectra of compound $\mathbf{2 b}$ ' 


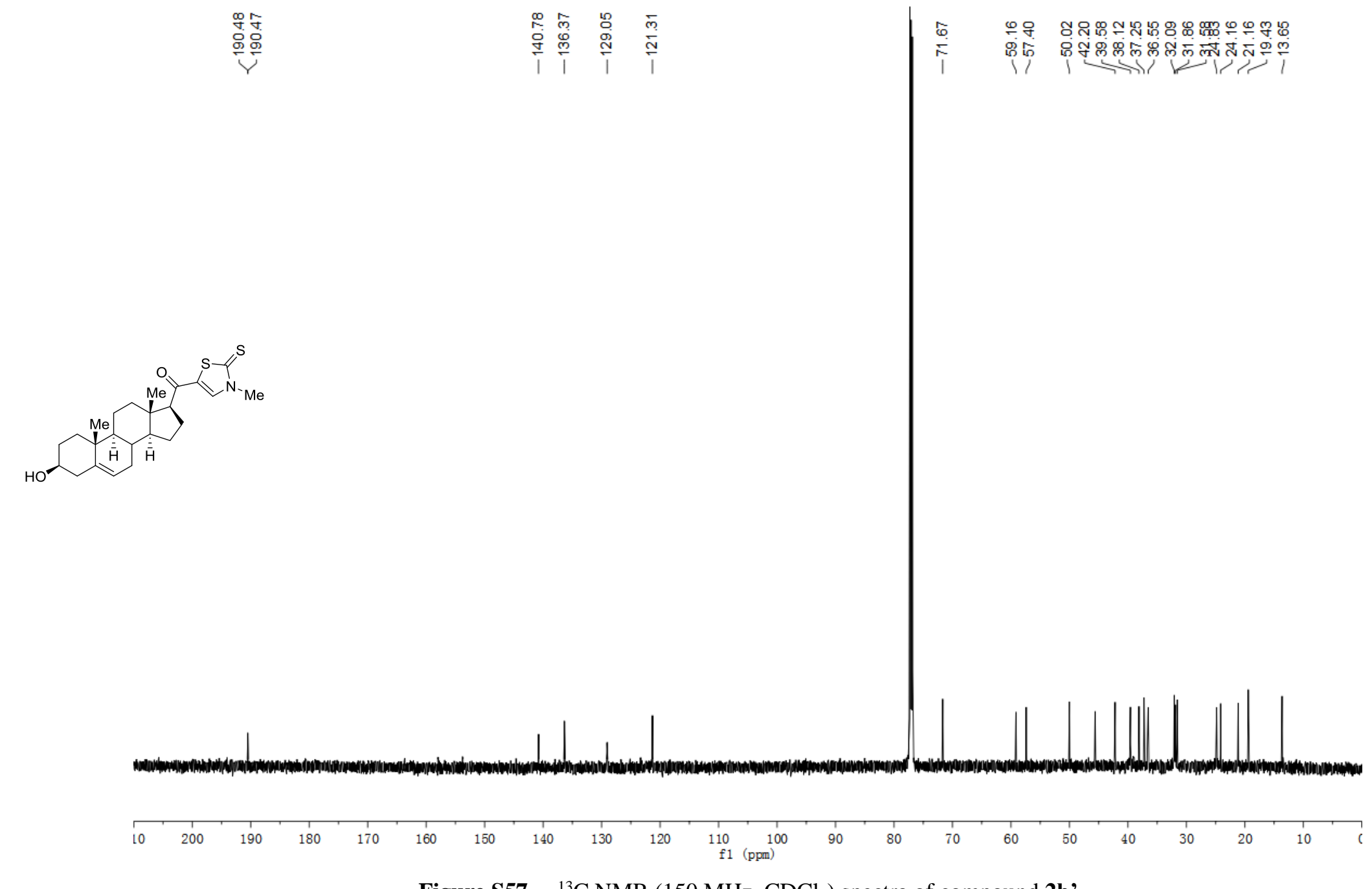

Figure S57. ${ }^{13} \mathrm{C}$ NMR $\left(150 \mathrm{MHz}, \mathrm{CDCl}_{3}\right)$ spectra of compound 2b' 
$\int_{M e}^{S}=s$

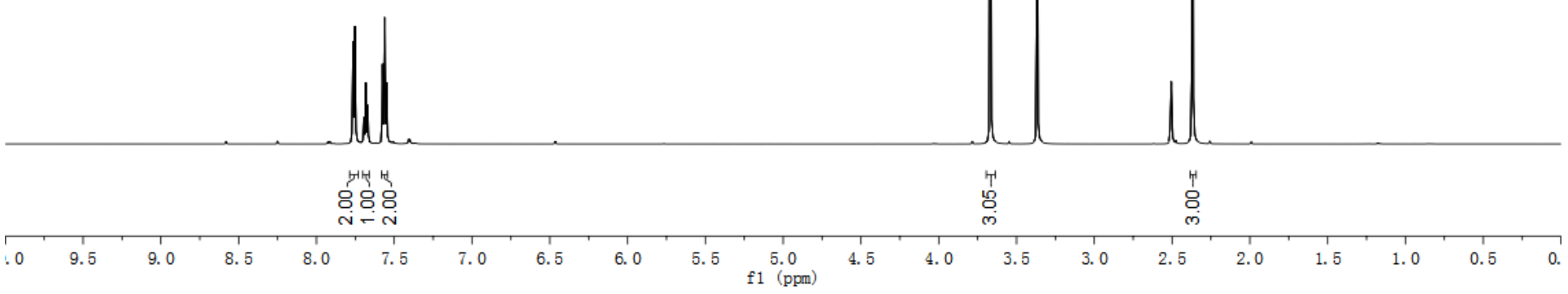

Figure S58. $\quad{ }^{1} \mathrm{H}$ NMR $\left(600 \mathrm{MHz}, \mathrm{DMSO}-d_{6}\right)$ spectra of compound $\mathbf{2 c}$ ' 


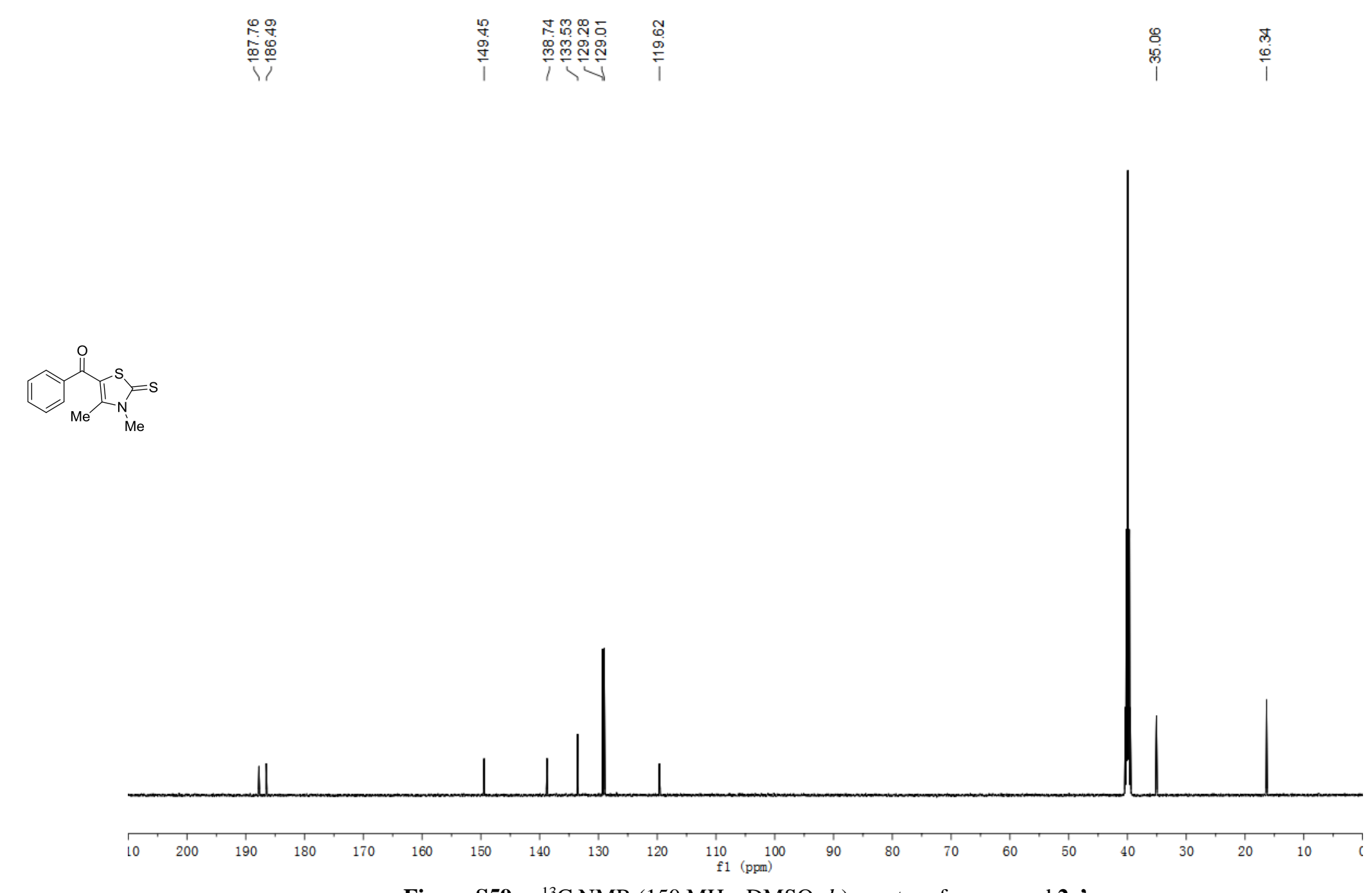

Figure S59. ${ }^{13} \mathrm{C}$ NMR $\left(150 \mathrm{MHz}, \mathrm{DMSO}-d_{6}\right)$ spectra of compound 2c' 
$\underbrace{S}_{P_{N}=S}=S$

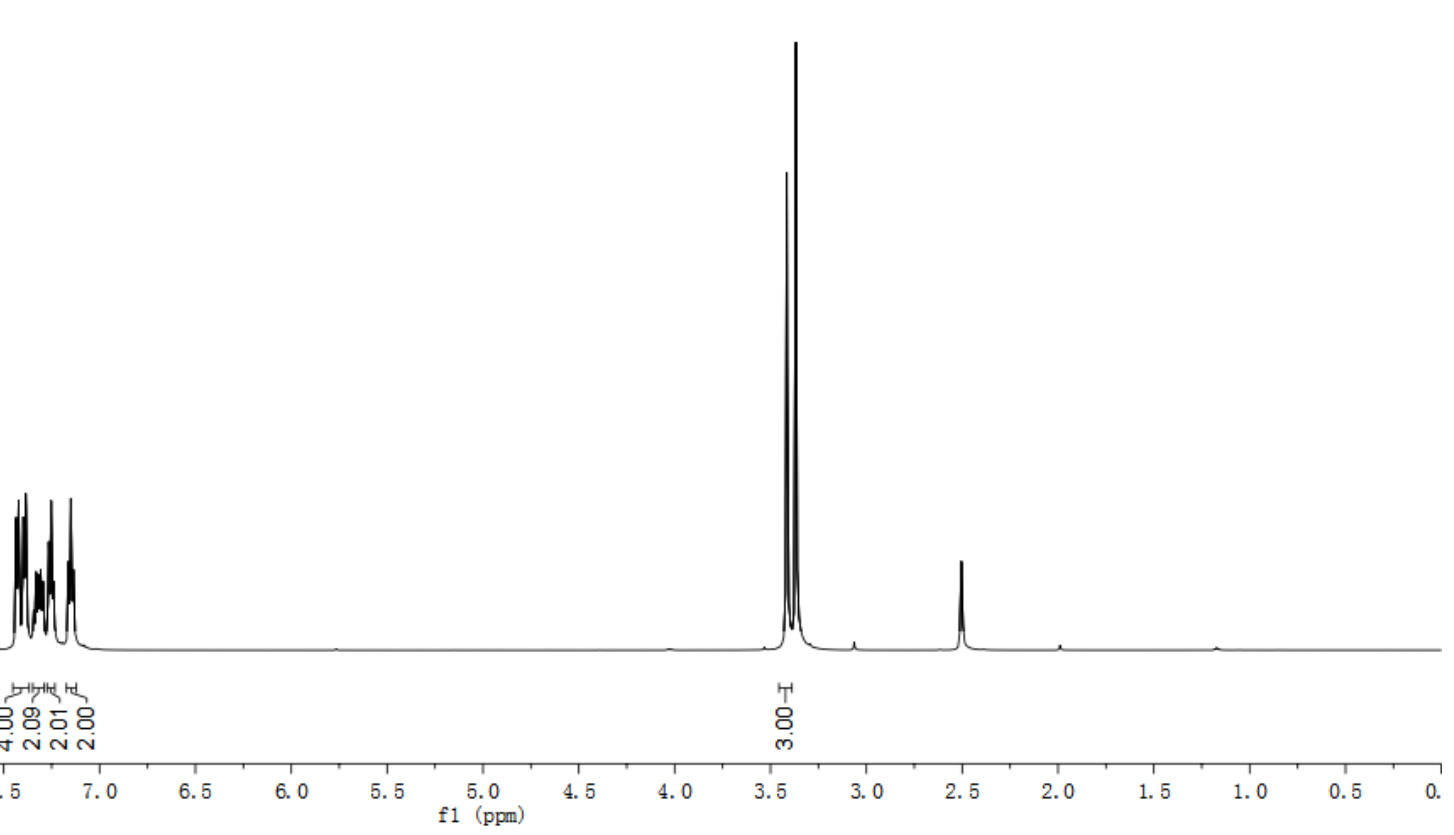

Figure S60. ${ }^{1} \mathrm{H}$ NMR $\left(600 \mathrm{MHz}, \mathrm{DMSO}-d_{6}\right)$ spectra of compound 2d' 


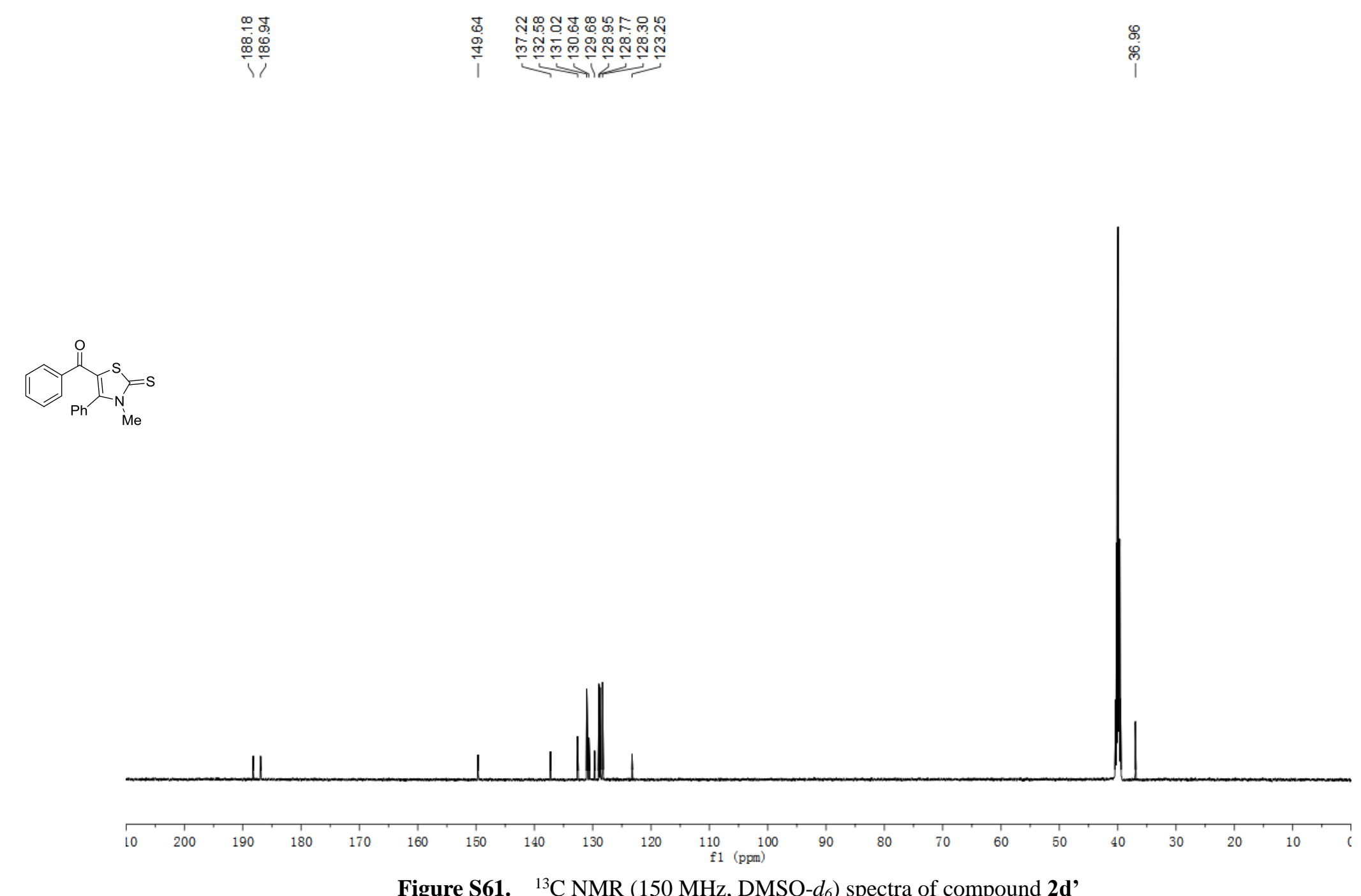

Figure S61. ${ }^{13} \mathrm{C}$ NMR $\left(150 \mathrm{MHz}\right.$, DMSO- $\left.d_{6}\right)$ spectra of compound 2d' 


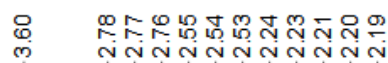

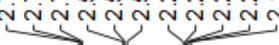

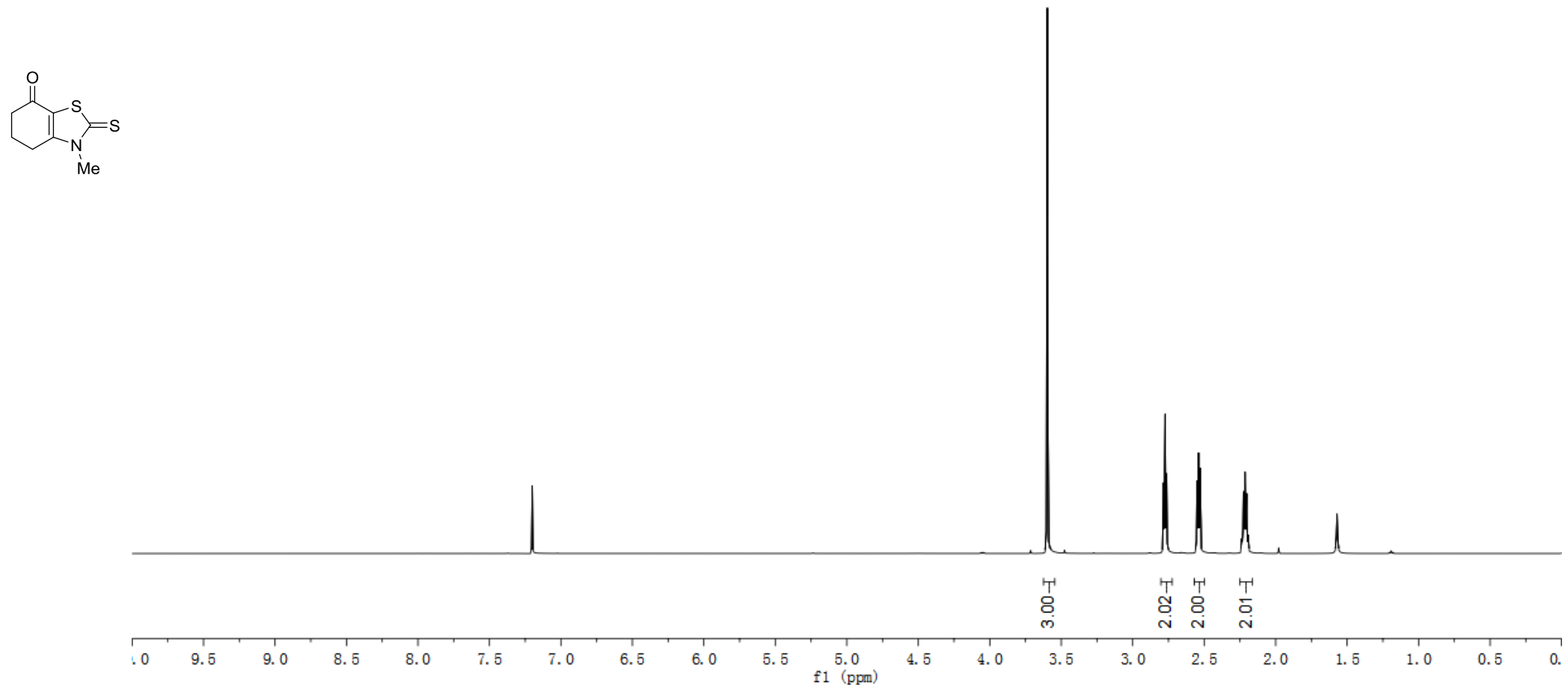

Figure S62. ' ${ }^{1} \mathrm{H}$ NMR (600 MHz, $\left.\mathrm{CDCl}_{3}\right)$ spectra of compound 2e' 


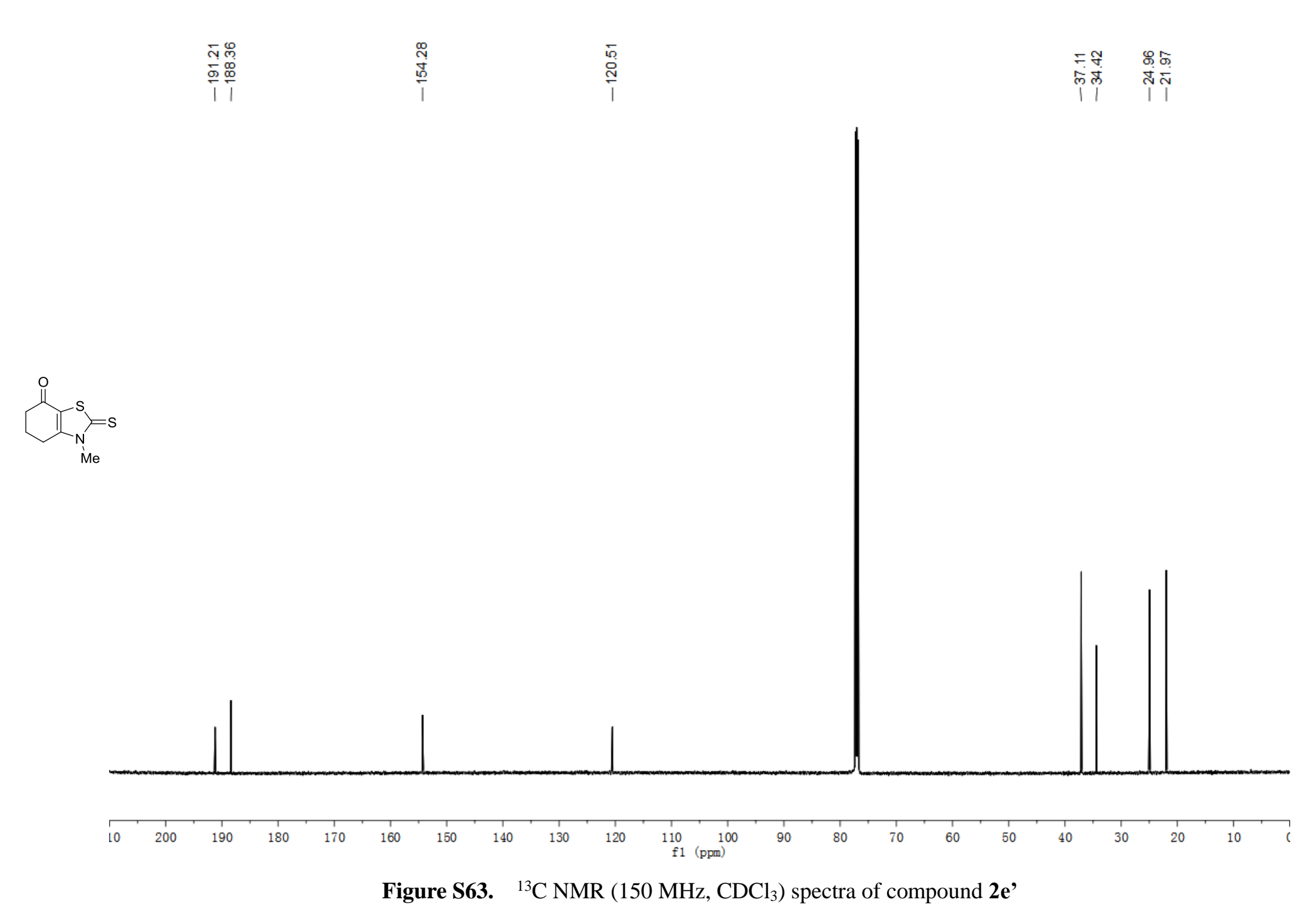



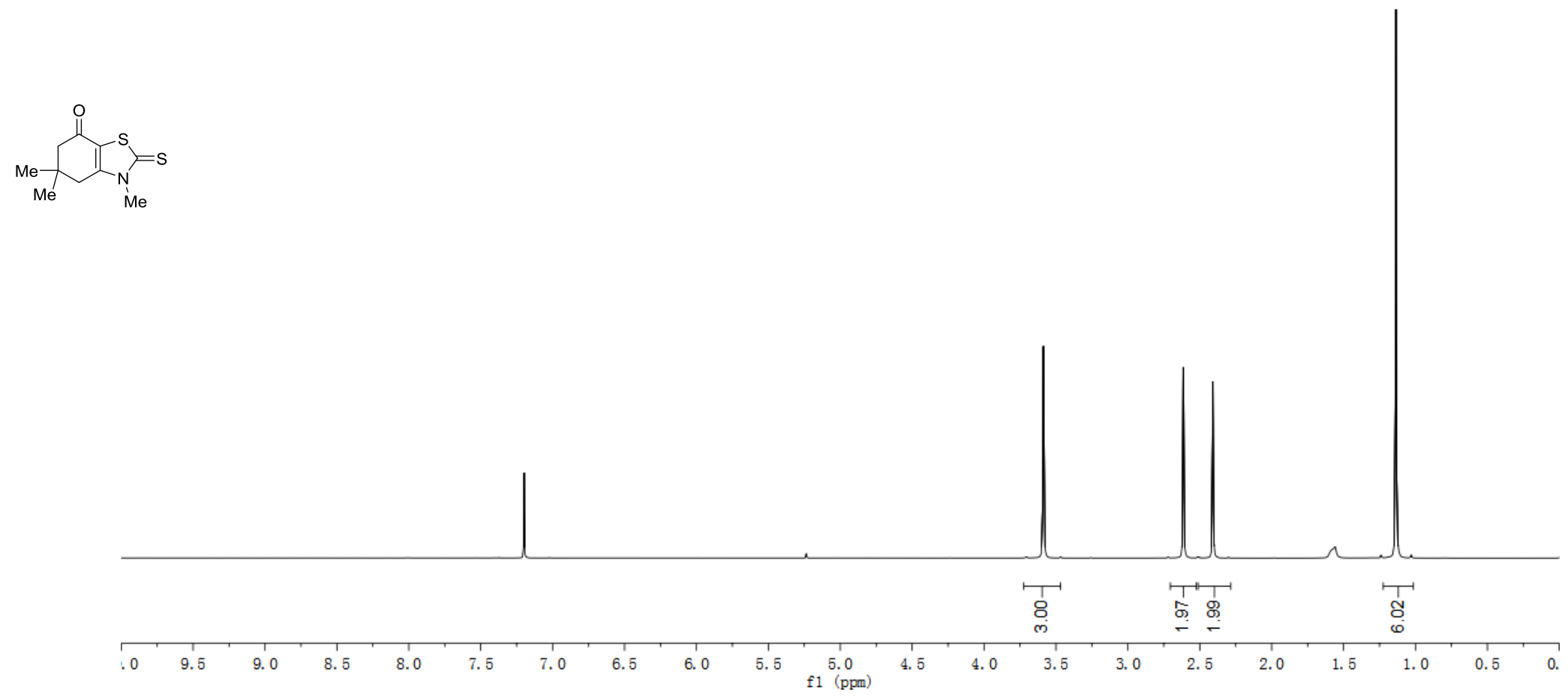

Figure S64. ' ${ }^{1} \mathrm{H}$ NMR (600 MHz, $\left.\mathrm{CDCl}_{3}\right)$ spectra of compound 2f' 


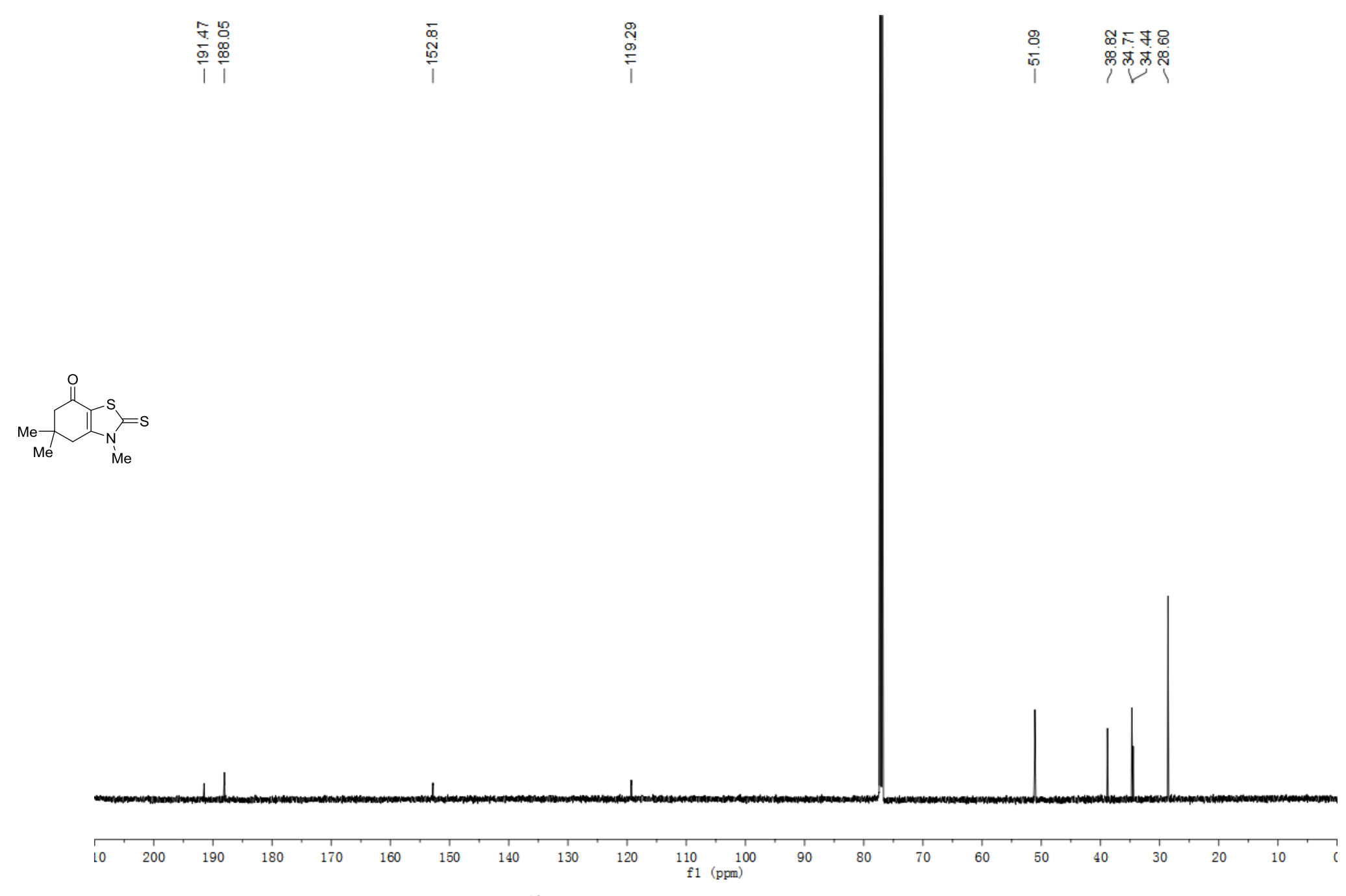

Figure S65. ${ }^{13} \mathrm{C} \mathrm{NMR}\left(150 \mathrm{MHz}, \mathrm{CDCl}_{3}\right)$ spectra of compound $\mathbf{2 f}$ ' 


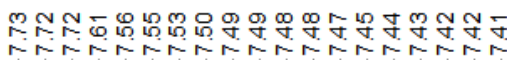

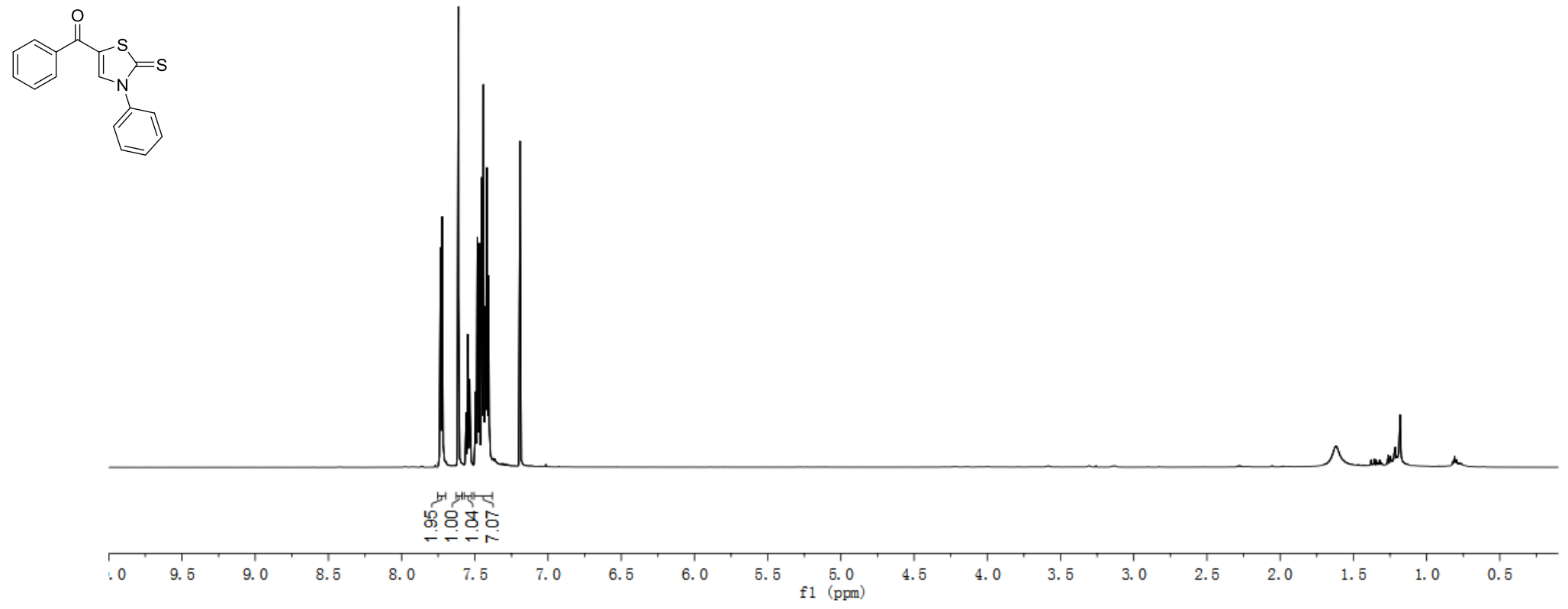

Figure S66. $\quad{ }^{1} \mathrm{H} \mathrm{NMR}\left(600 \mathrm{MHz}, \mathrm{CDCl}_{3}\right)$ spectra of compound $4 \mathbf{a}$ 


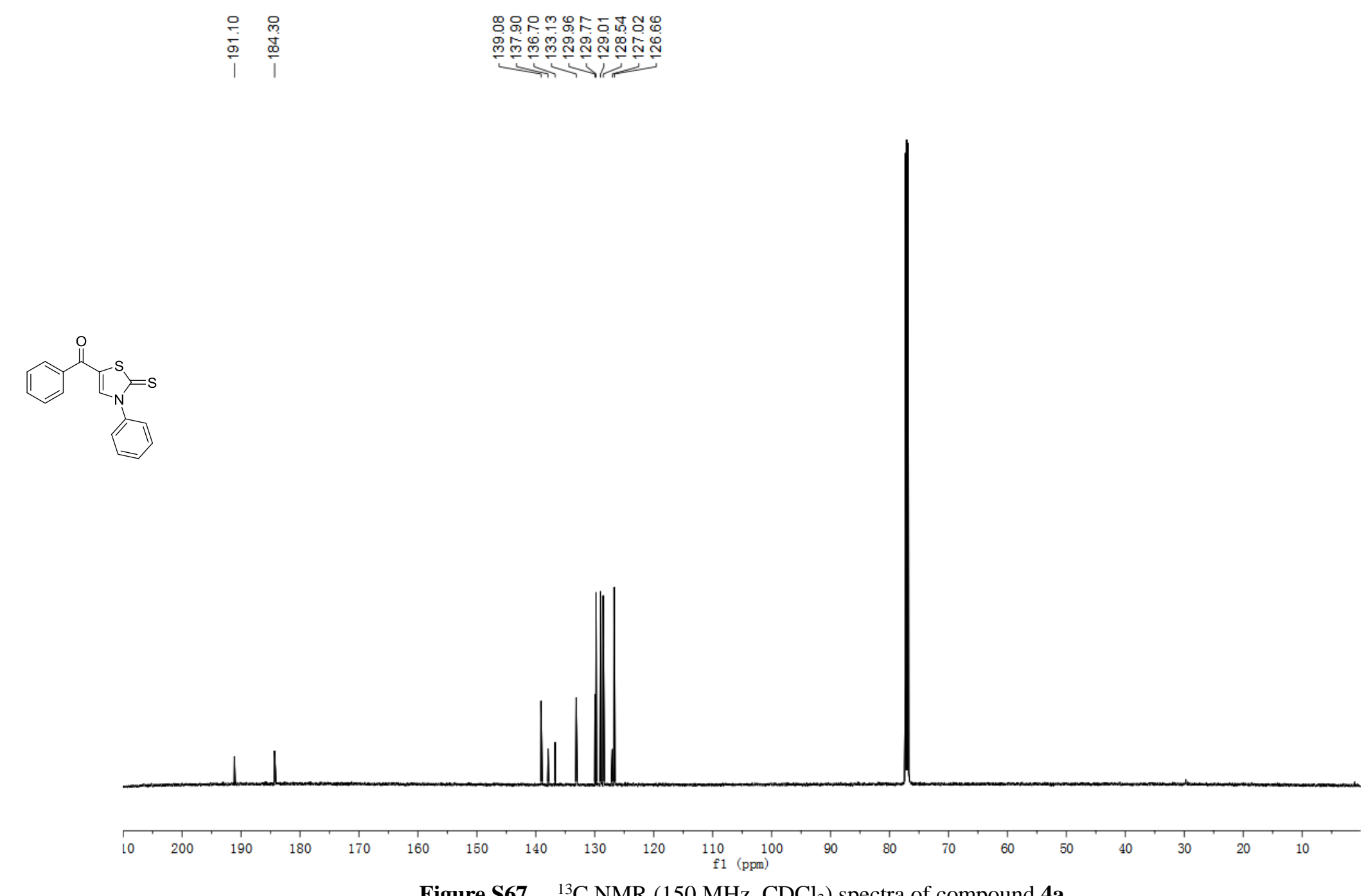

Figure S67. ${ }^{13} \mathrm{C} \mathrm{NMR}\left(150 \mathrm{MHz}, \mathrm{CDCl}_{3}\right)$ spectra of compound $4 \mathbf{a}$ 


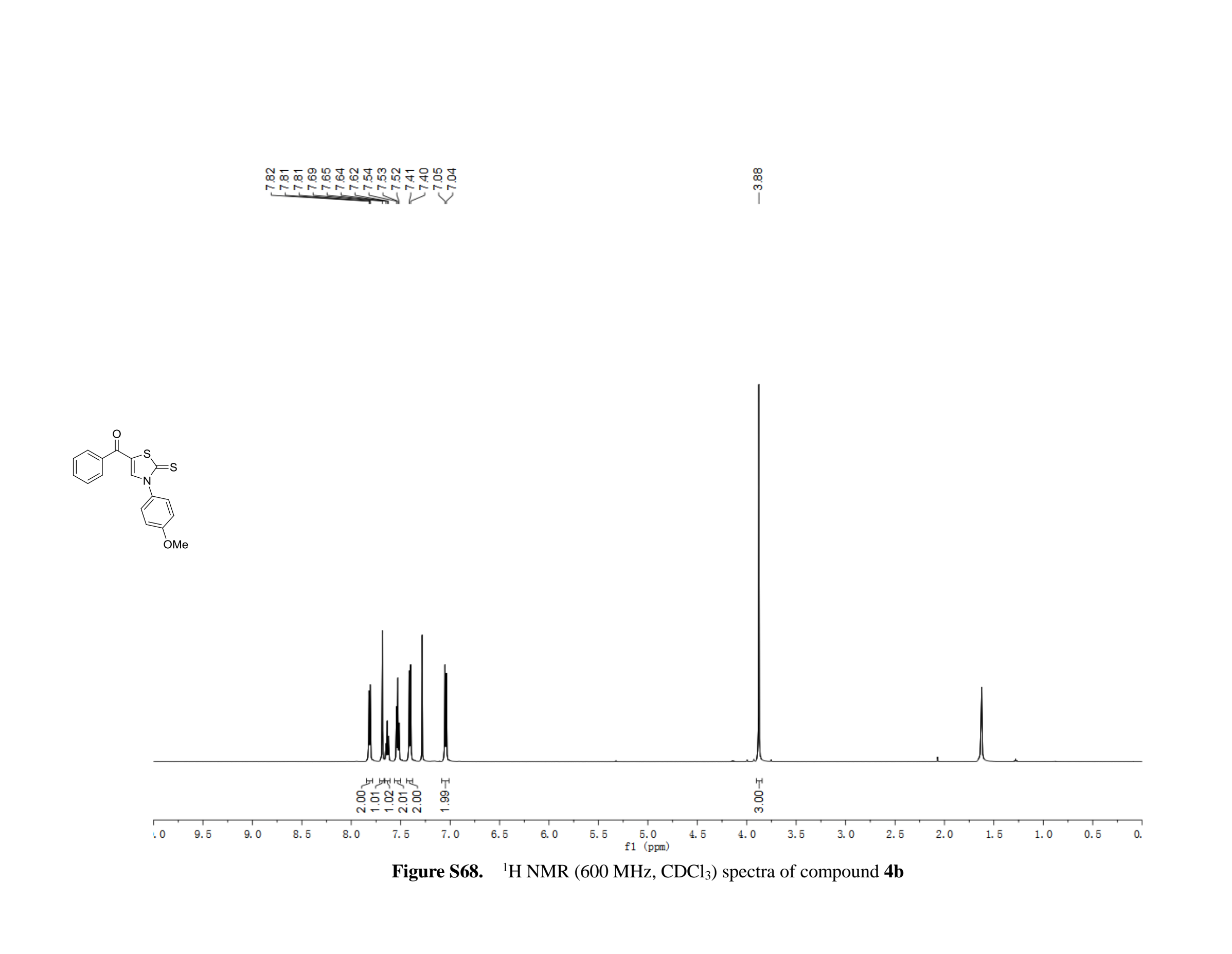




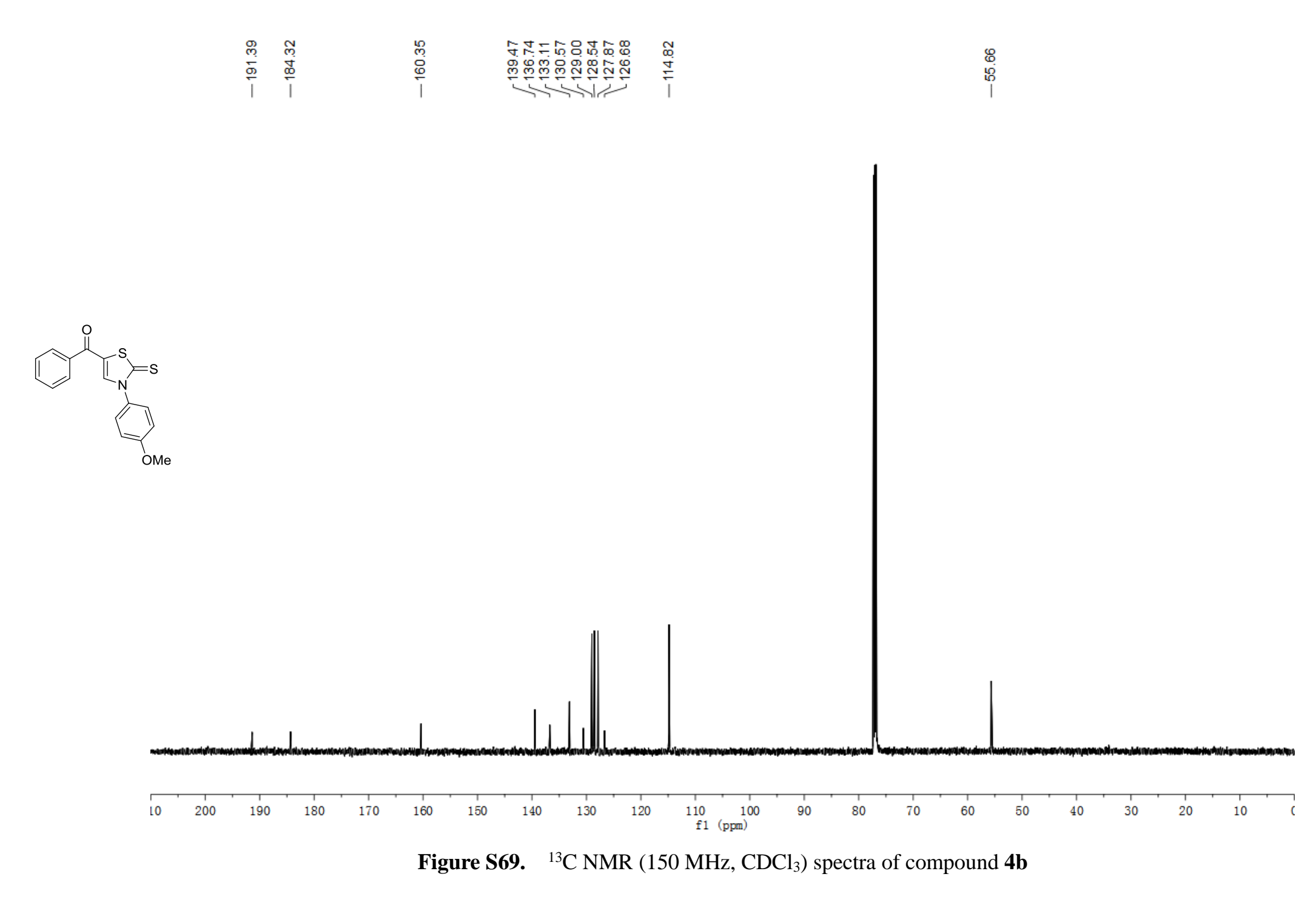



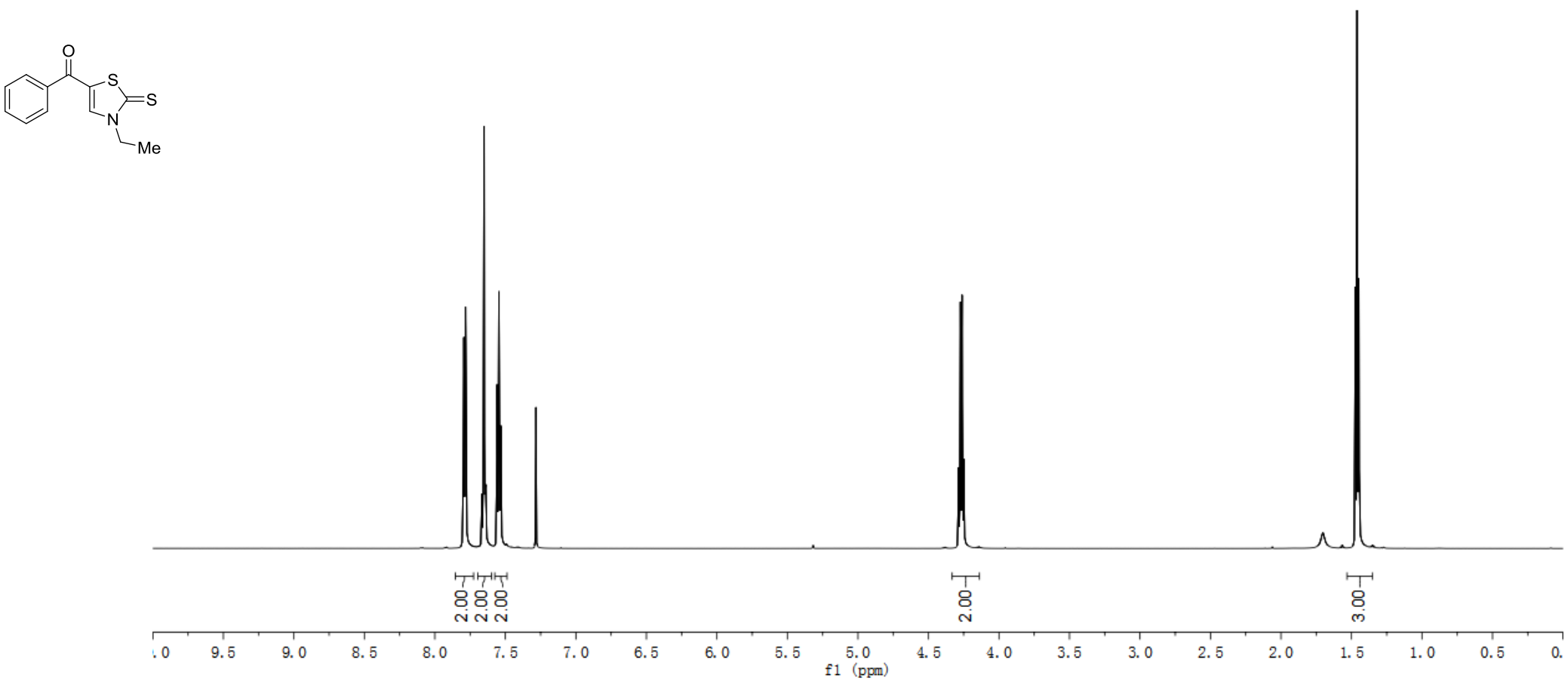

Figure S70. $\quad{ }^{1} \mathrm{H}$ NMR $\left(600 \mathrm{MHz}, \mathrm{CDCl}_{3}\right)$ spectra of compound $4 \mathbf{c}$ 


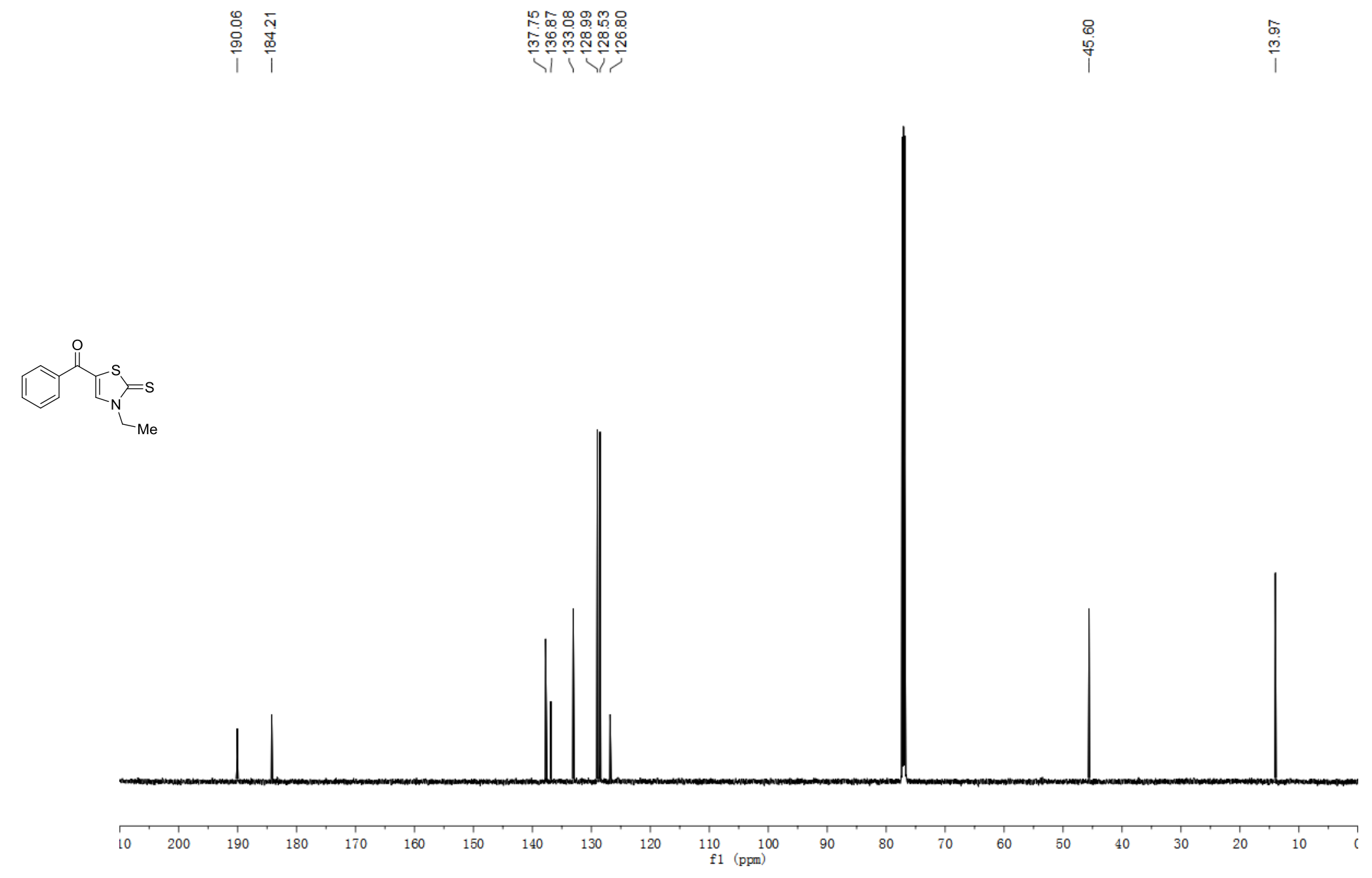

Figure S71. ${ }^{13} \mathrm{C}$ NMR $\left(150 \mathrm{MHz}, \mathrm{CDCl}_{3}\right)$ spectra of compound $4 \mathbf{c}$ 

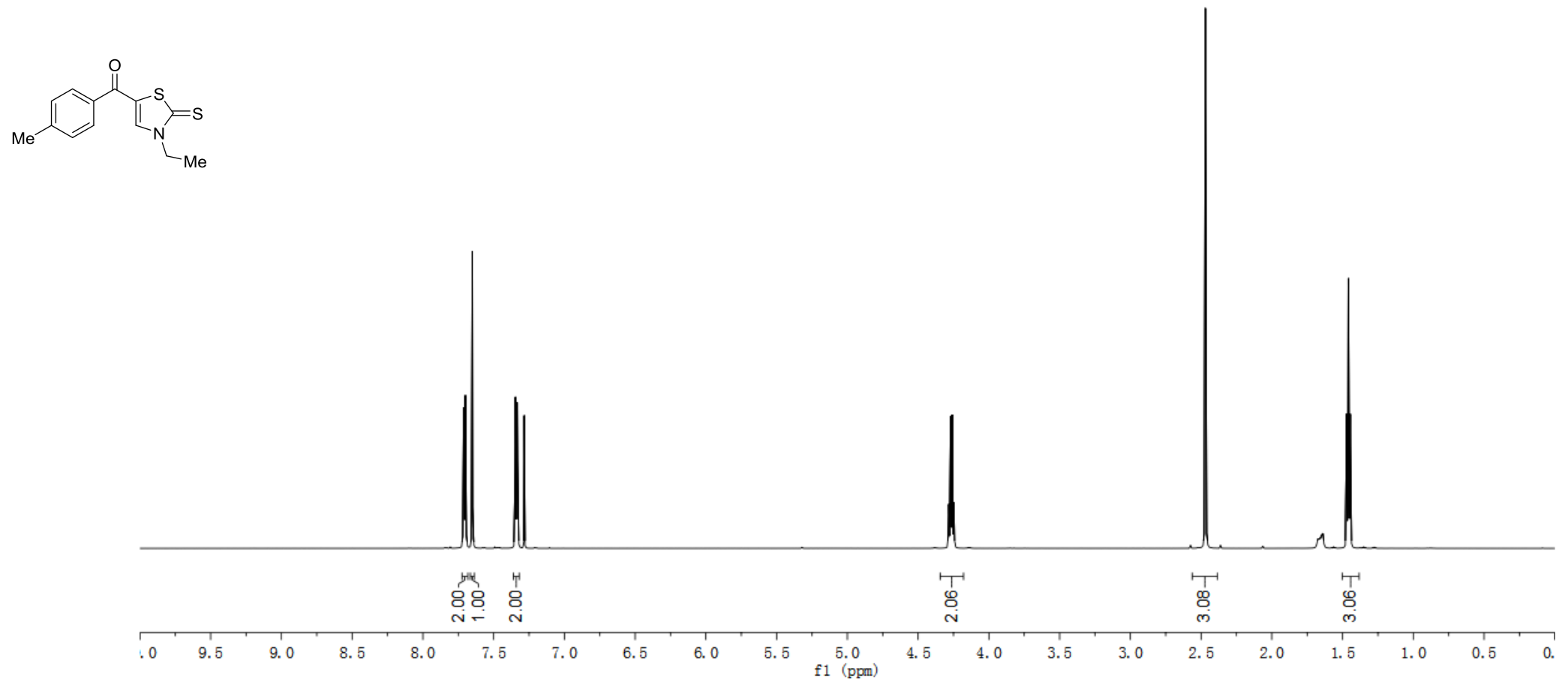

Figure S72. $\quad{ }^{1} \mathrm{H}$ NMR (600 MHz, $\left.\mathrm{CDCl}_{3}\right)$ spectra of compound 4d 


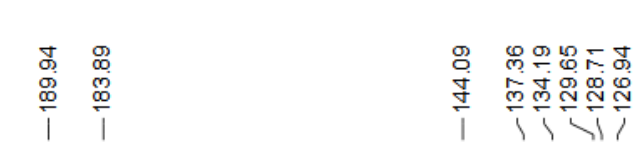

量早量

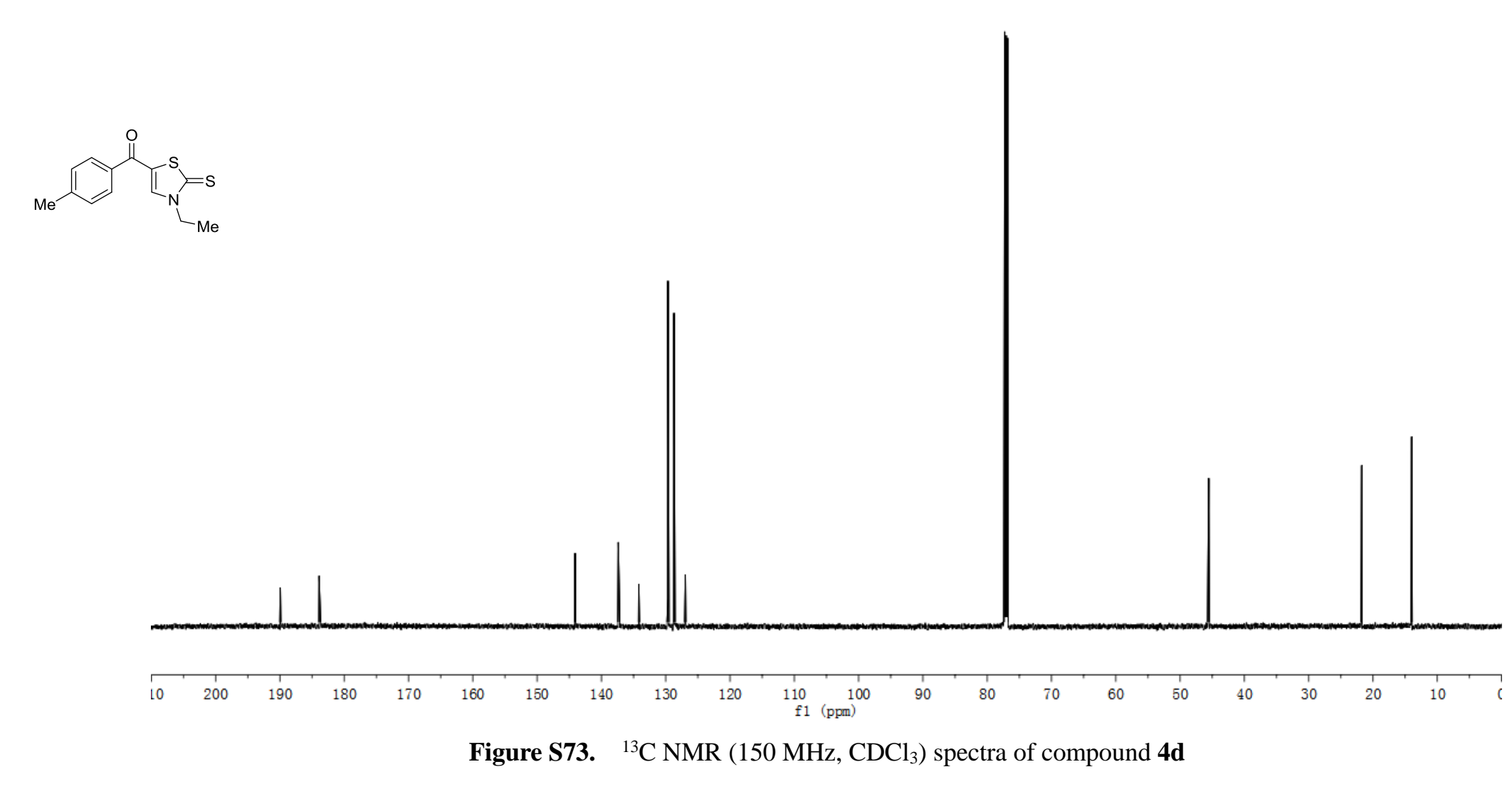


${ }_{N_{N}^{S}}^{s}=s$
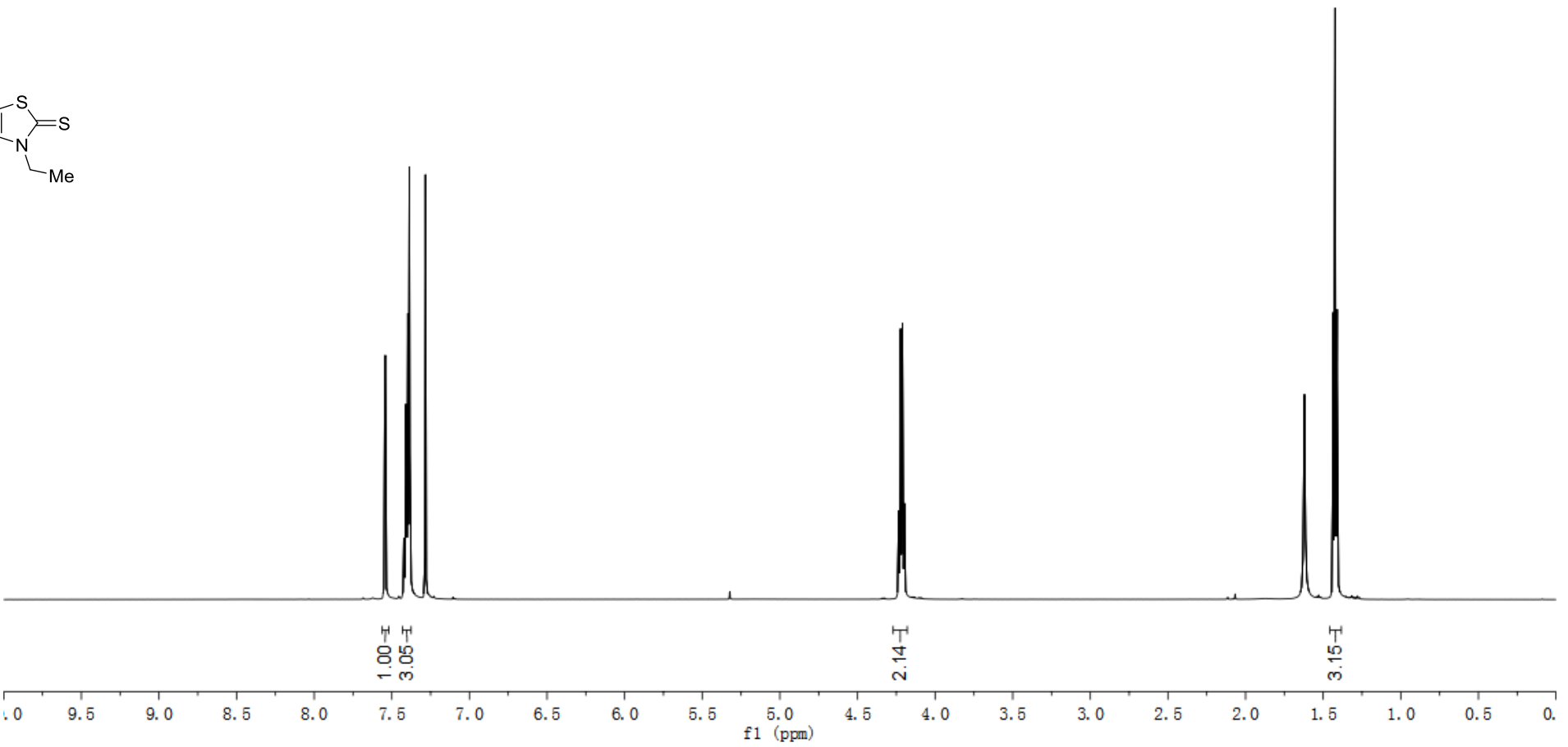

Figure S74. ${ }^{1} \mathrm{H} \mathrm{NMR}\left(600 \mathrm{MHz}, \mathrm{CDCl}_{3}\right)$ spectra of compound $4 \mathbf{e}$ 


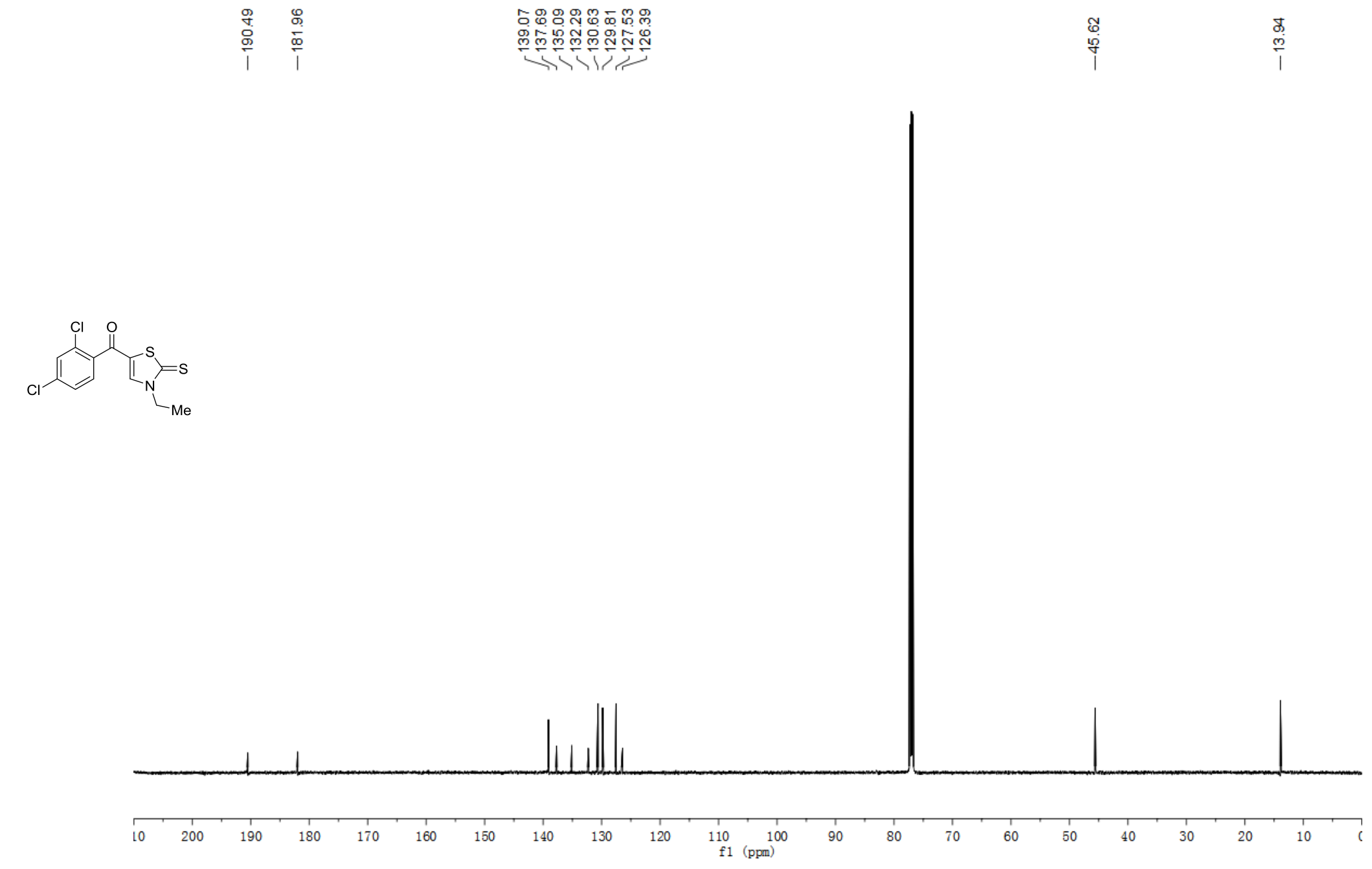

Figure S75. ${ }^{13} \mathrm{C} \mathrm{NMR}\left(150 \mathrm{MHz}, \mathrm{CDCl}_{3}\right)$ spectra of compound $4 \mathbf{e}$ 


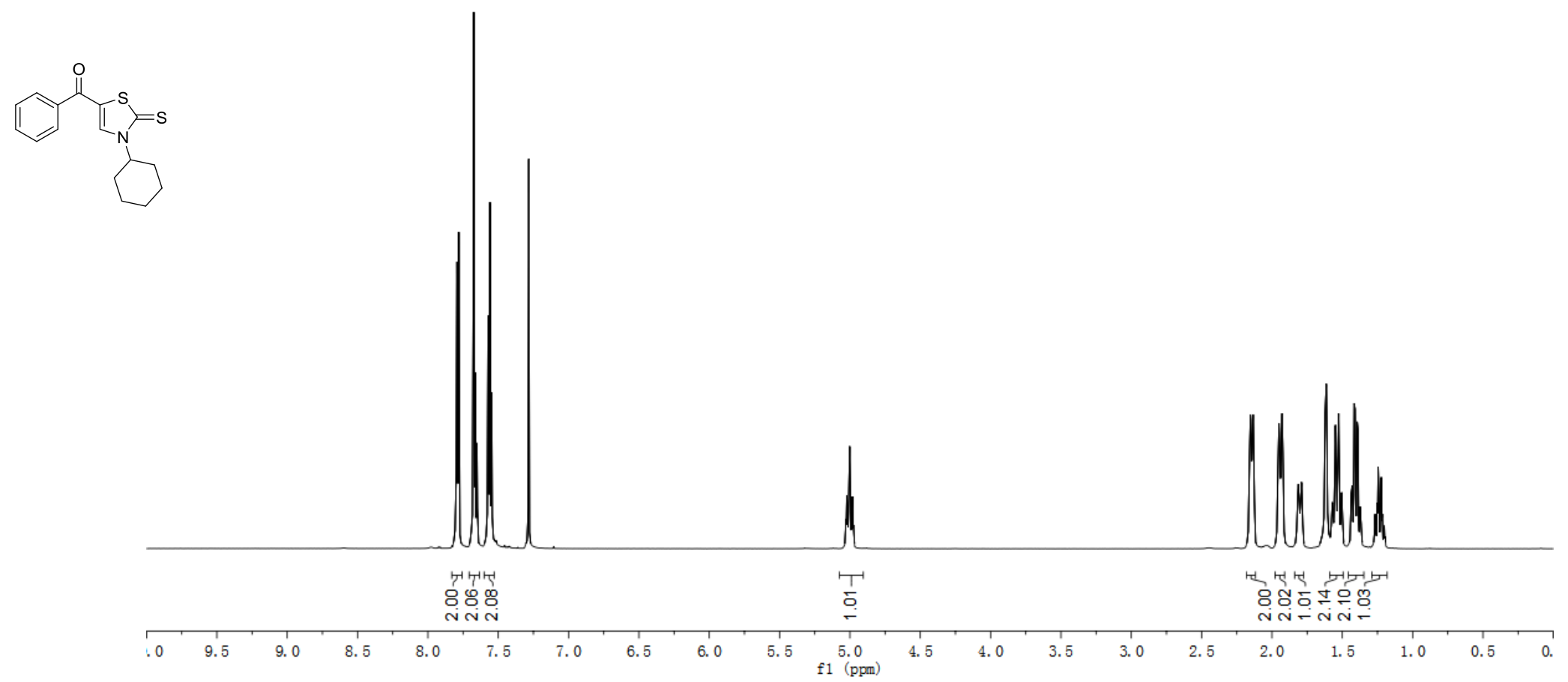

Figure S76. $\quad{ }^{1} \mathrm{H}$ NMR $\left(600 \mathrm{MHz}, \mathrm{CDCl}_{3}\right)$ spectra of compound $\mathbf{4 f}$ 
$\underset{\substack{\sigma \\ \infty}}{\substack{\infty \\ \infty}}$
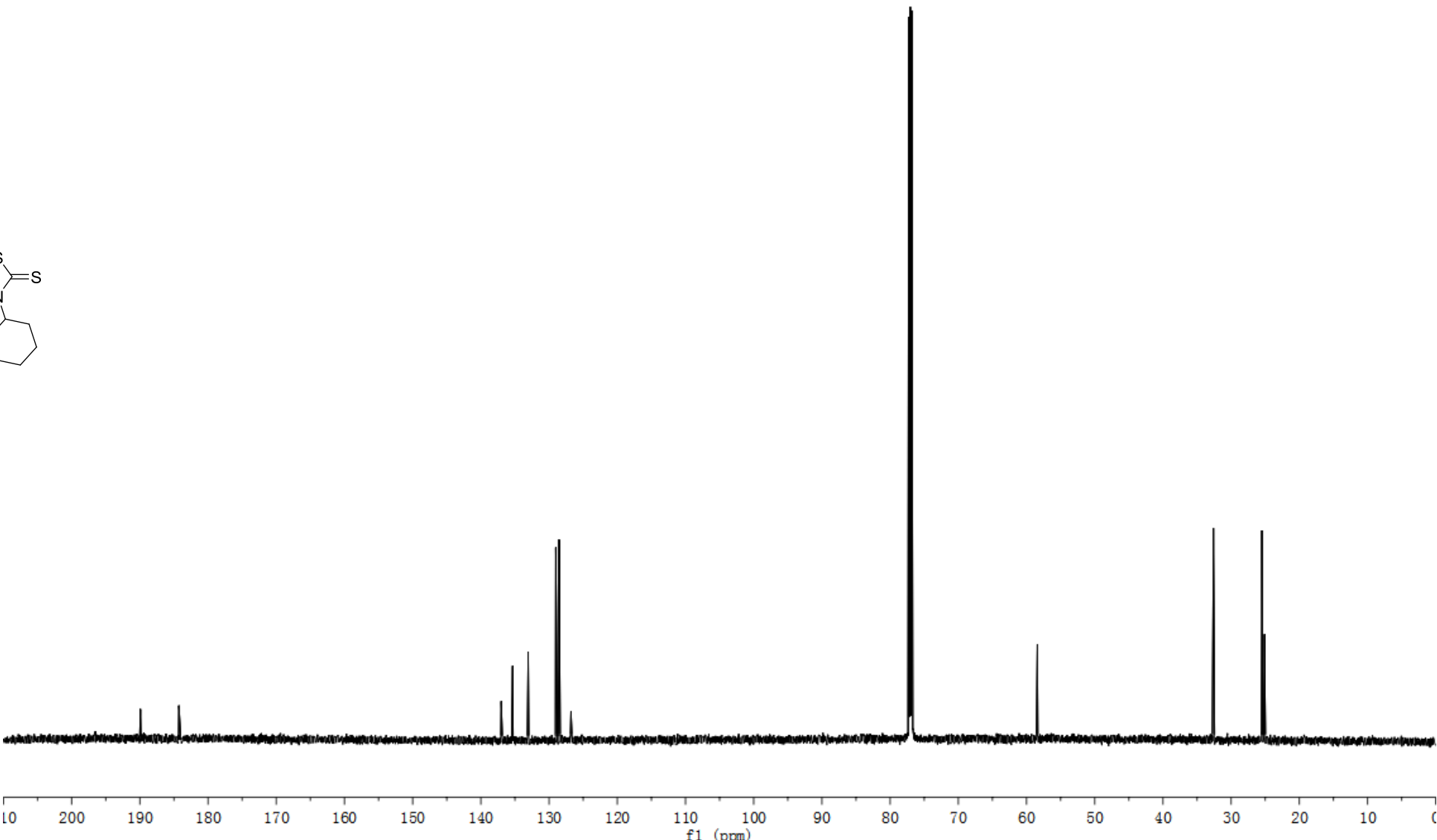

$\begin{array}{cc}110 & 100 \\ \text { f1 } & (\mathrm{ppm})\end{array}$

Figure S77. ${ }^{13} \mathrm{C} \mathrm{NMR}\left(150 \mathrm{MHz}, \mathrm{CDCl}_{3}\right)$ spectra of compound $\mathbf{4 f}$ 


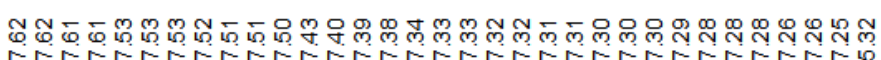
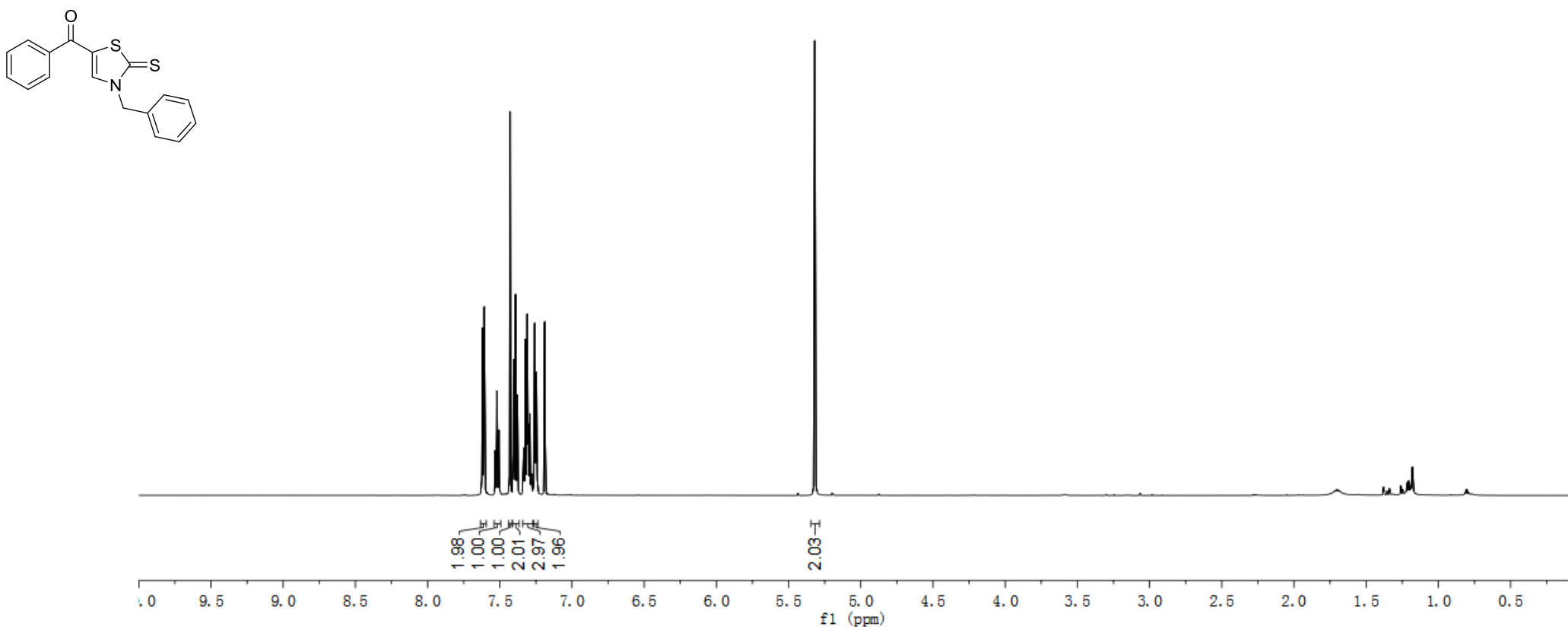

Figure S78. ${ }^{1} \mathrm{H}$ NMR $\left(600 \mathrm{MHz}, \mathrm{CDCl}_{3}\right)$ spectra of compound $\mathbf{4 g}$ 


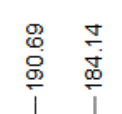

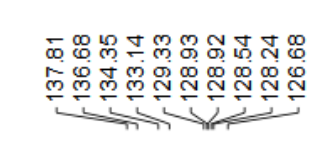

$\frac{2}{19}$

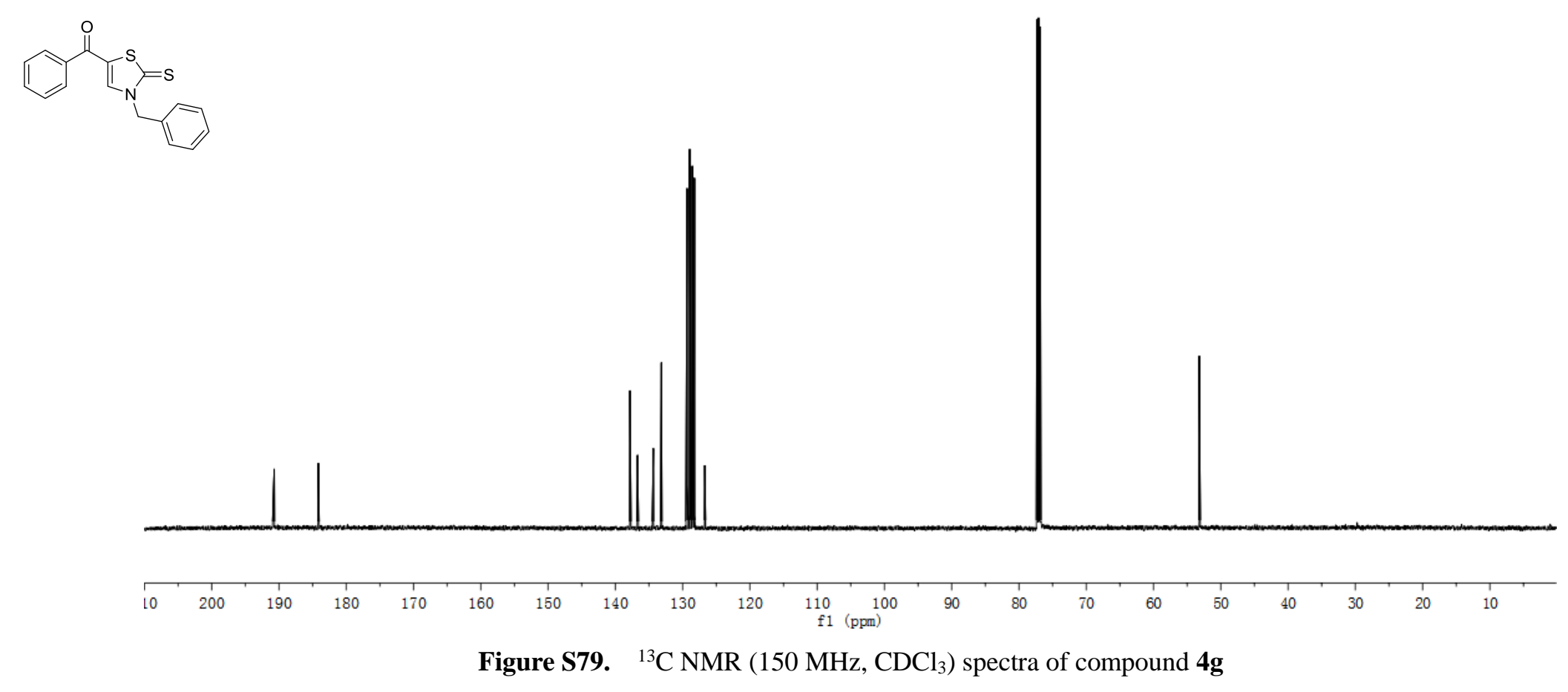



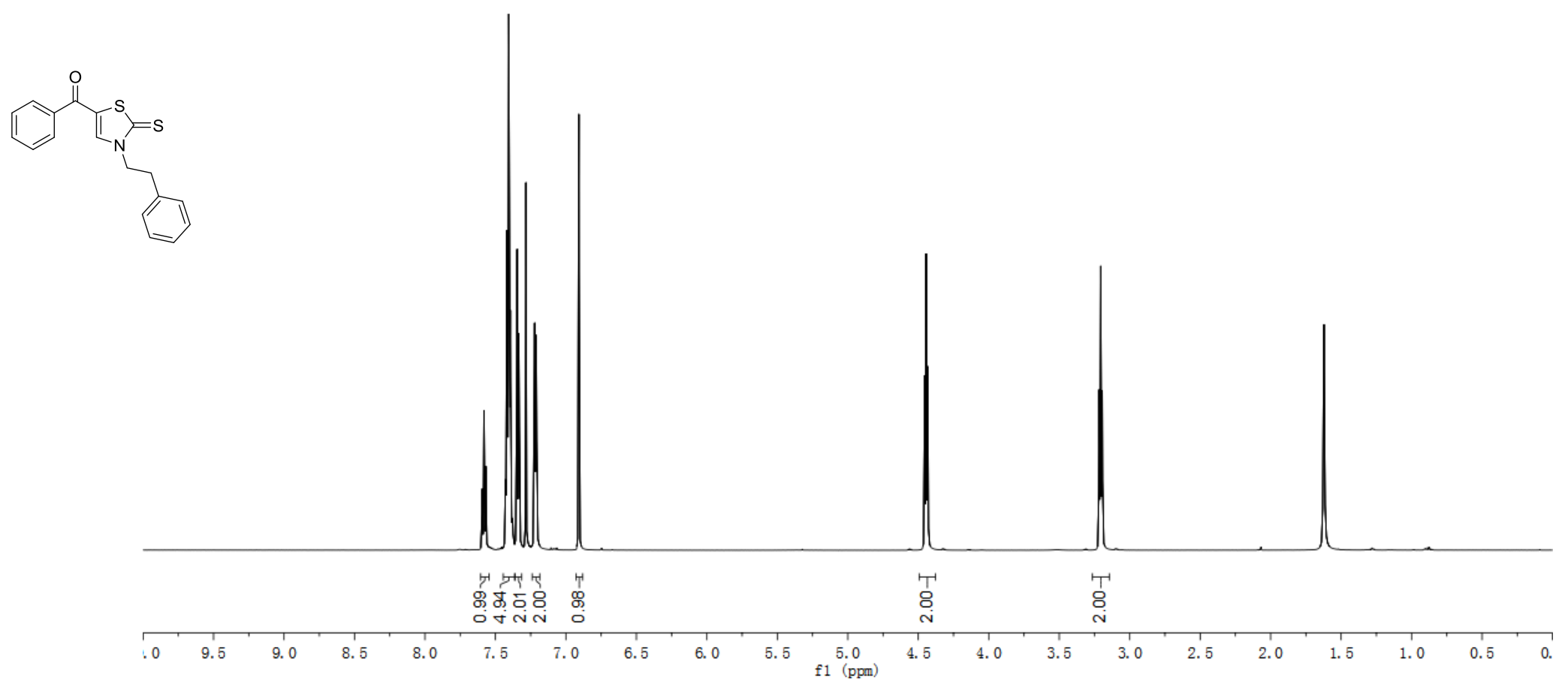

Figure S80. ${ }^{1} \mathrm{H}$ NMR $\left(600 \mathrm{MHz}, \mathrm{CDCl}_{3}\right)$ spectra of compound $\mathbf{4 h}$ 
\}

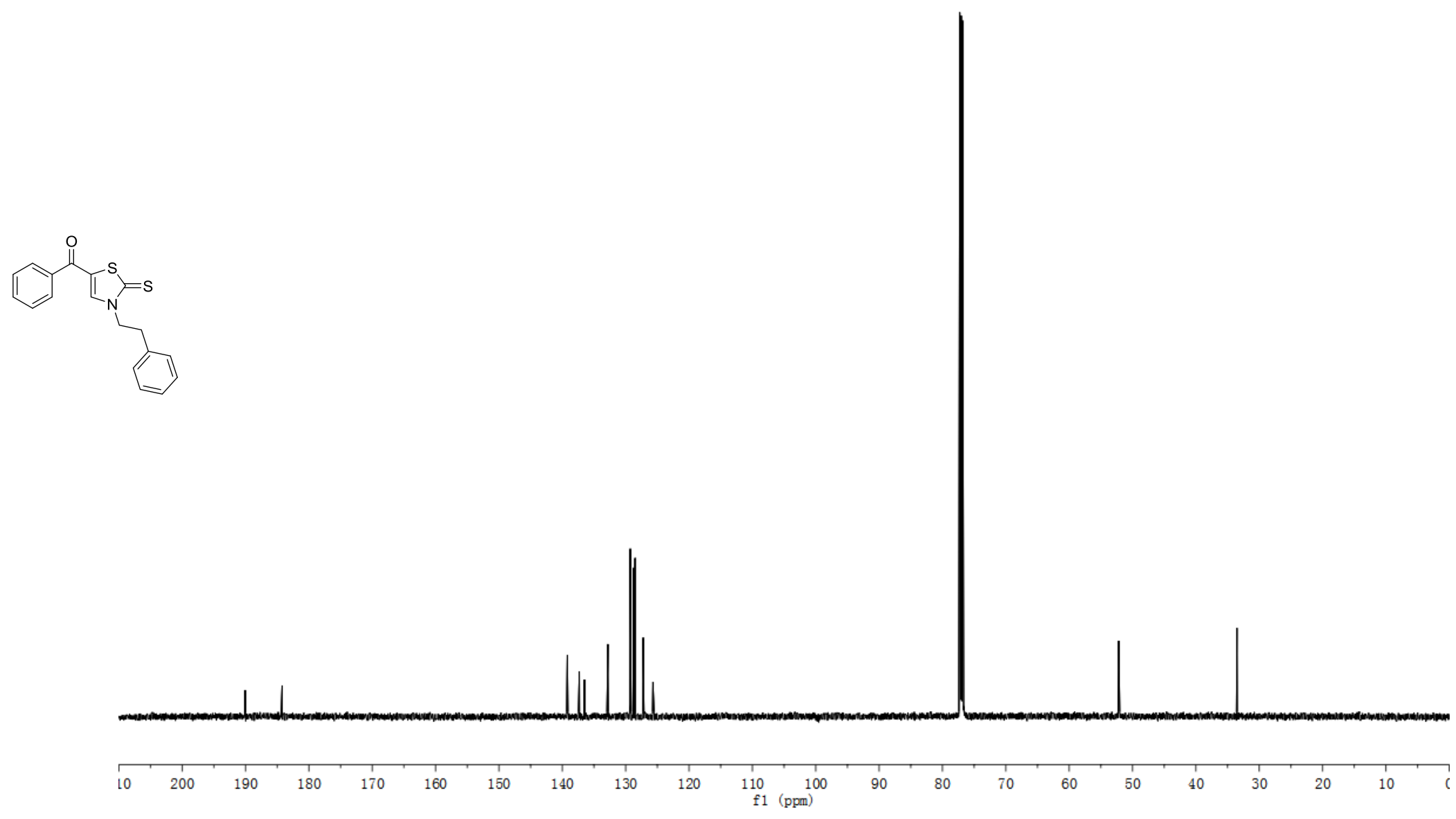

Figure S81. ${ }^{13} \mathrm{C} \mathrm{NMR}\left(150 \mathrm{MHz}, \mathrm{CDCl}_{3}\right)$ spectra of compound $\mathbf{4 h}$ 

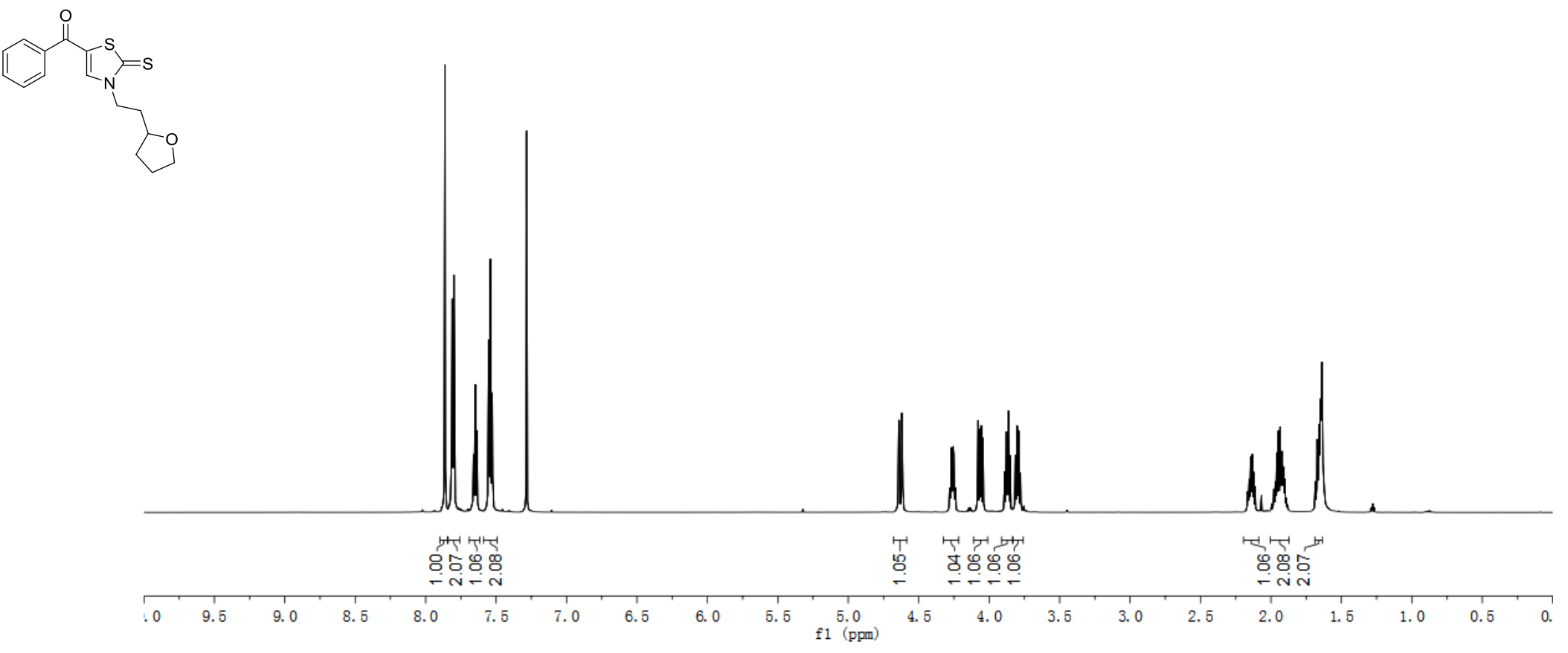

Figure S82. ${ }^{1} \mathrm{H} \mathrm{NMR}\left(600 \mathrm{MHz}, \mathrm{CDCl}_{3}\right)$ spectra of compound $4 \mathbf{i}$ 

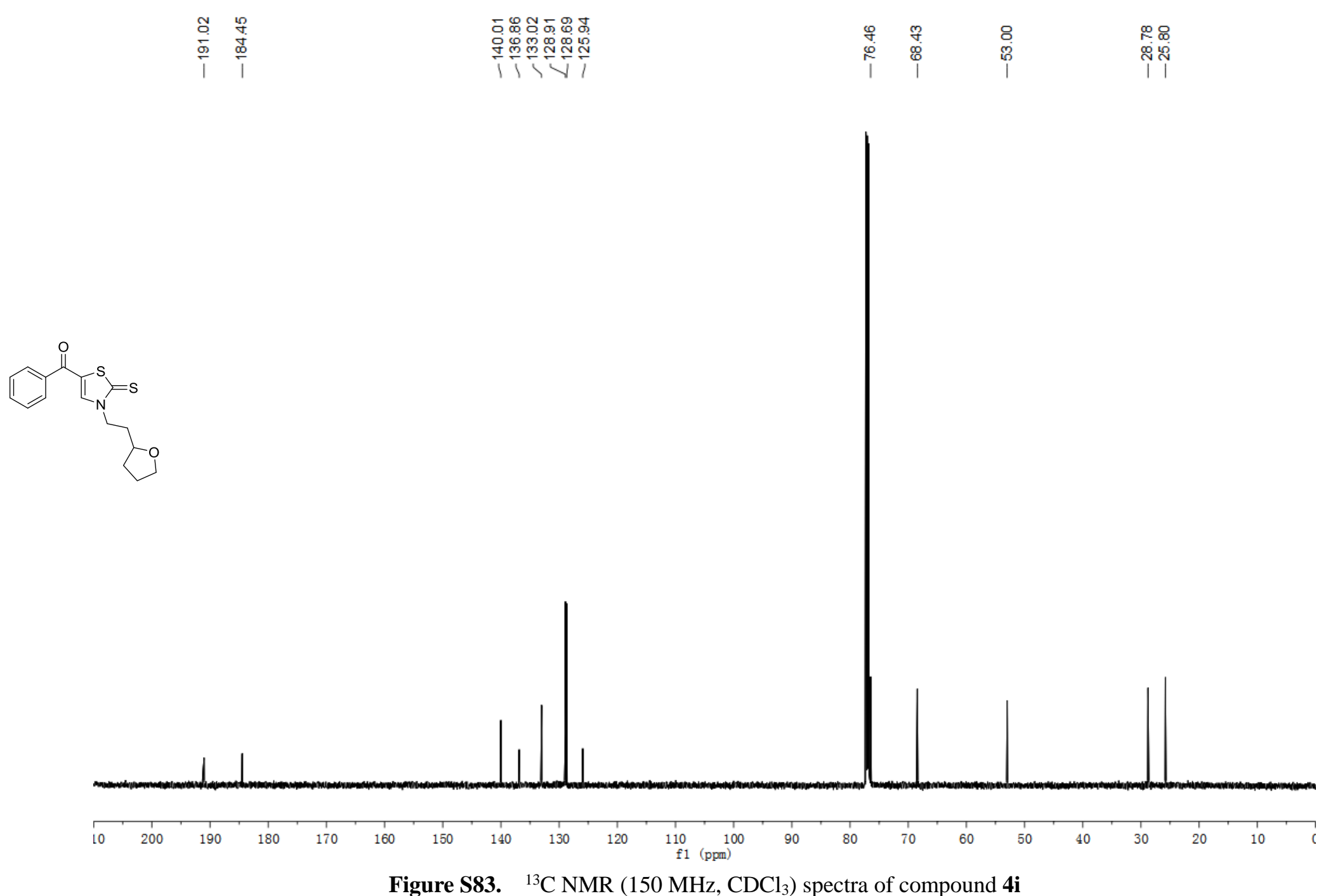

Figure S83. ${ }^{13} \mathrm{C} \mathrm{NMR}\left(150 \mathrm{MHz}, \mathrm{CDCl}_{3}\right)$ spectra of compound $4 \mathbf{i}$ 


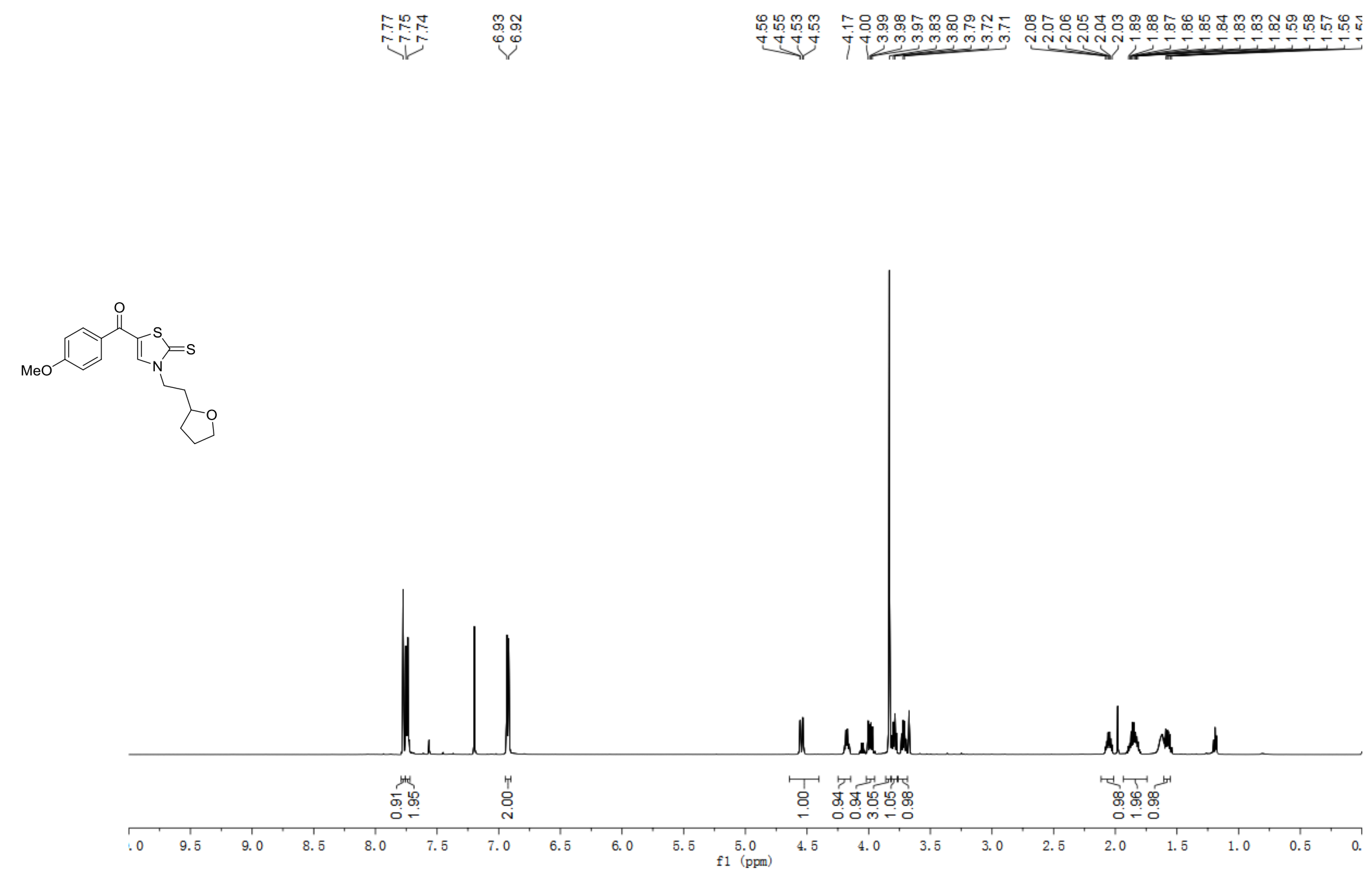

Figure S84. ${ }^{1} \mathrm{H} \mathrm{NMR}\left(600 \mathrm{MHz}, \mathrm{CDCl}_{3}\right)$ spectra of compound $\mathbf{4 j}$ 


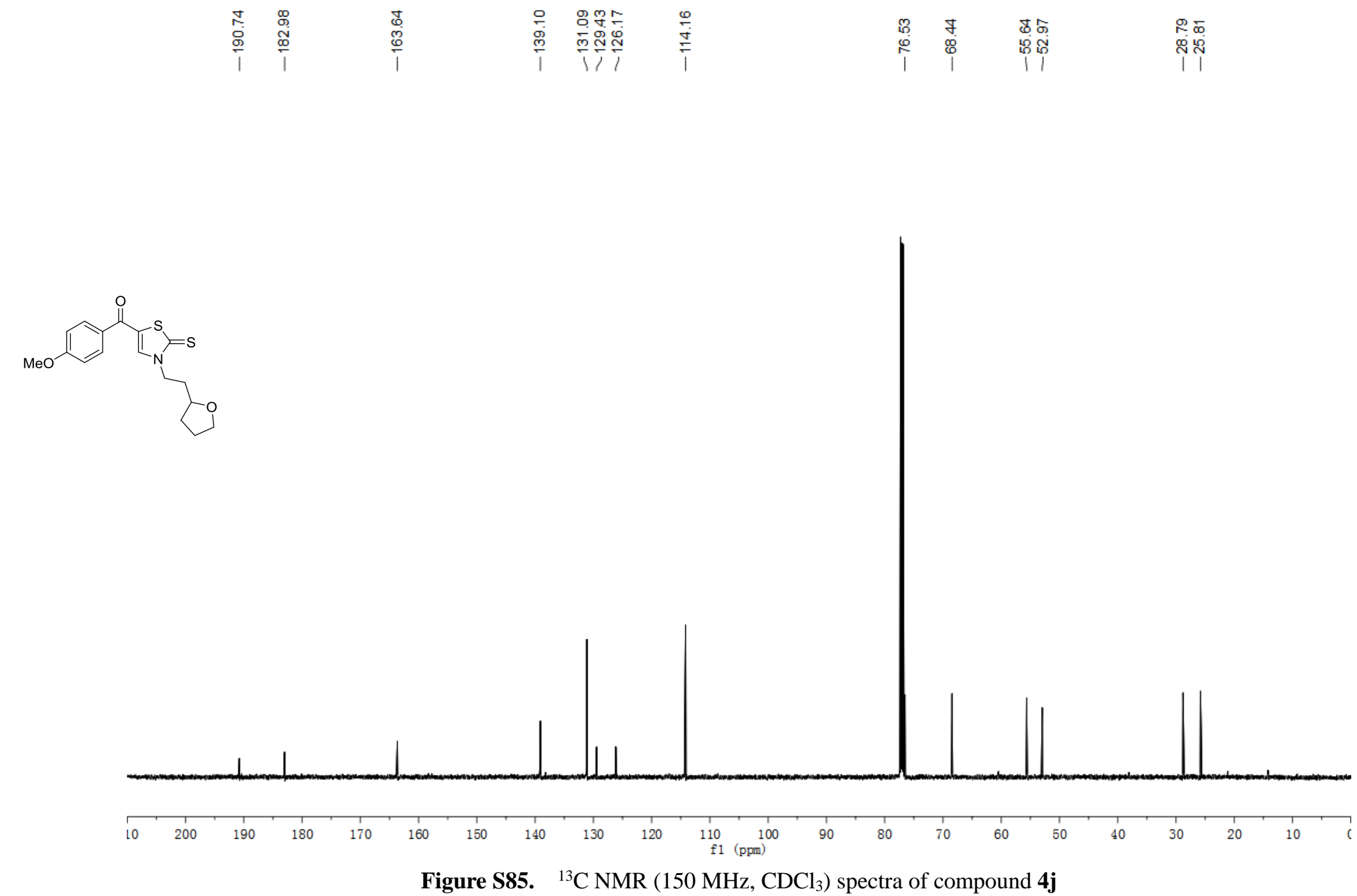



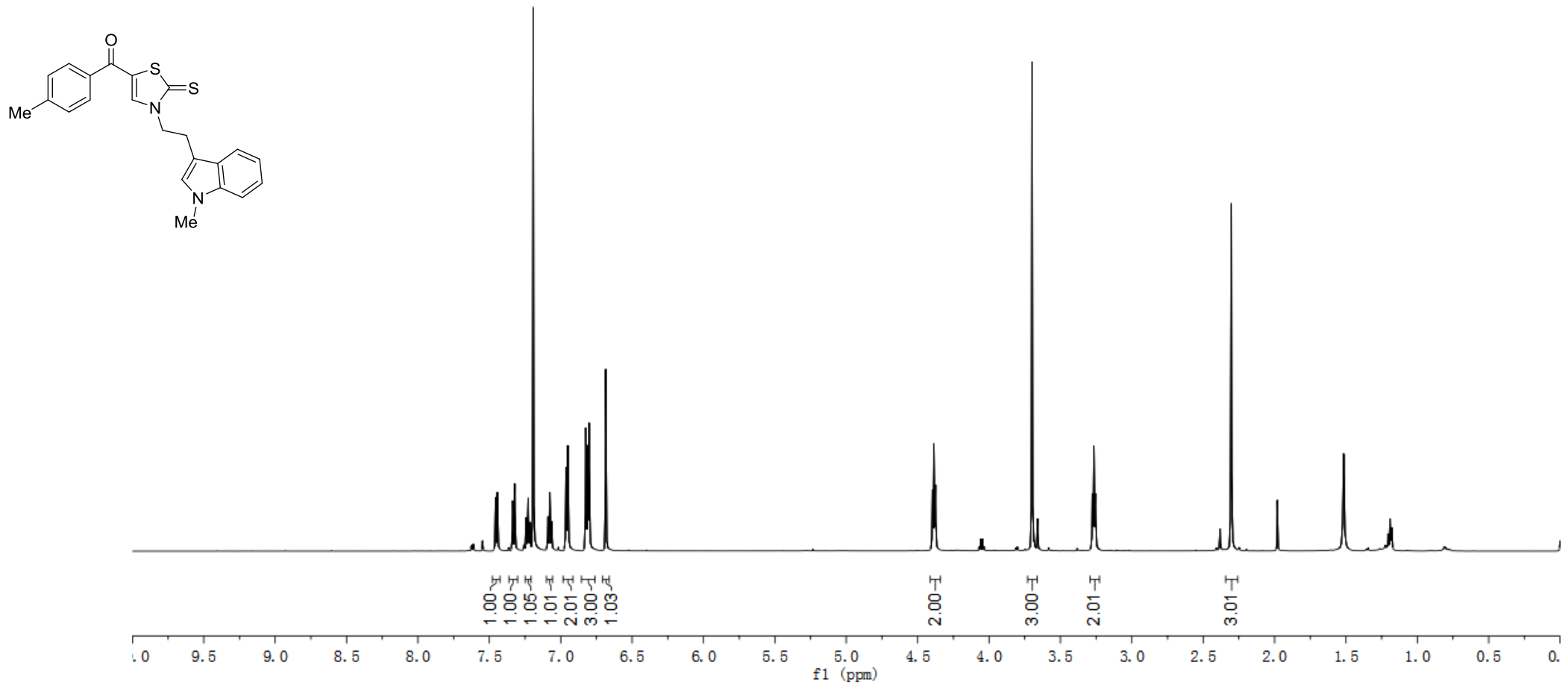

Figure S86. $\quad{ }^{1} \mathrm{H}$ NMR $\left(600 \mathrm{MHz}, \mathrm{CDCl}_{3}\right)$ spectra of compound $\mathbf{4 k}$ 

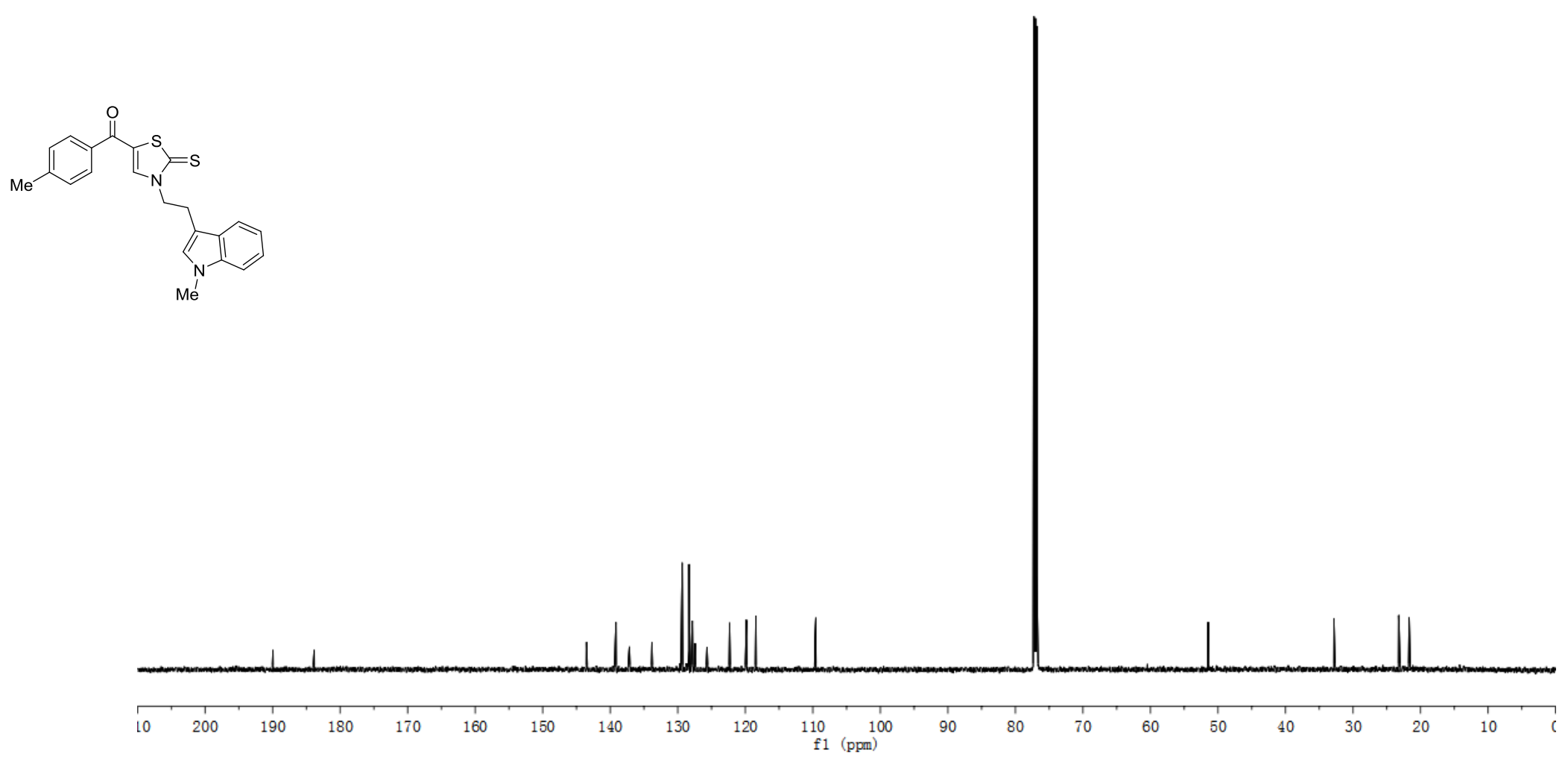

Figure S87. ${ }^{13} \mathrm{C}$ NMR $\left(150 \mathrm{MHz}, \mathrm{CDCl}_{3}\right)$ spectra of compound $4 \mathbf{k}$ 

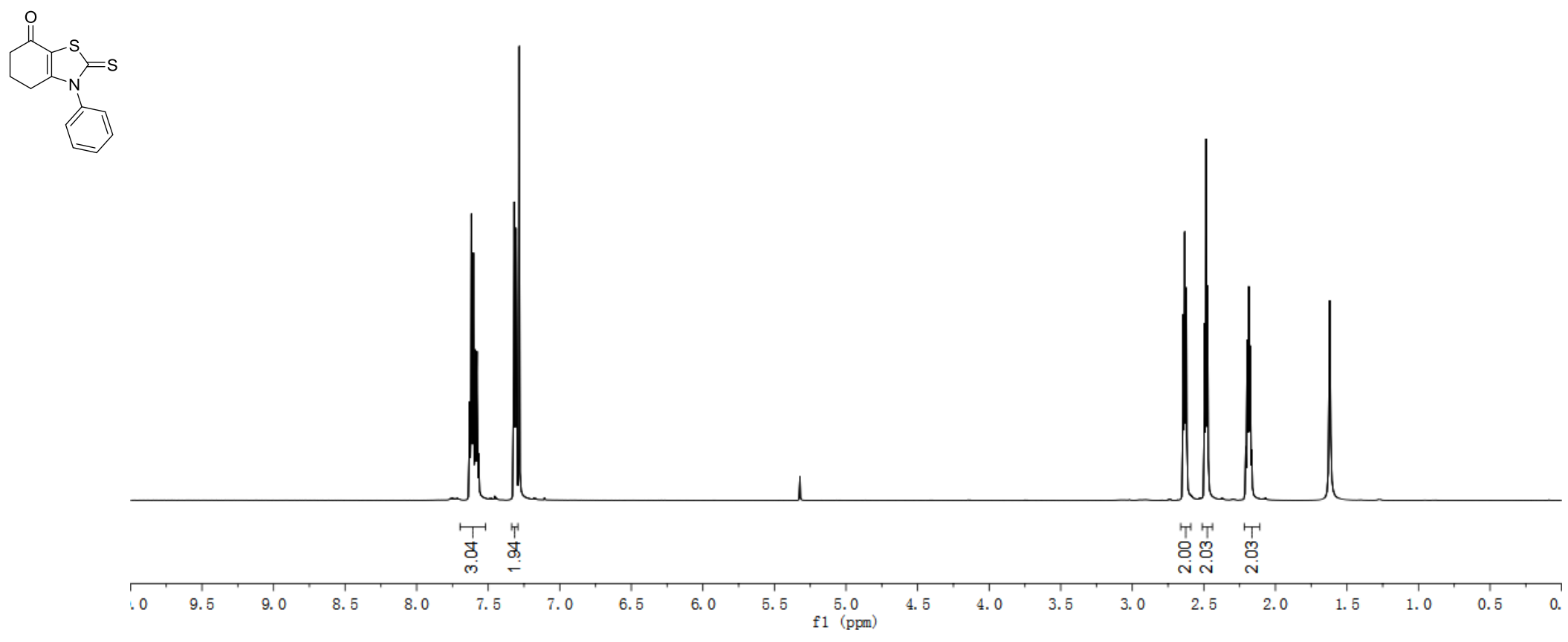

Figure S88. $\quad{ }^{1} \mathrm{H} \mathrm{NMR}\left(600 \mathrm{MHz}, \mathrm{CDCl}_{3}\right)$ spectra of compound $4 \mathbf{l}$ 


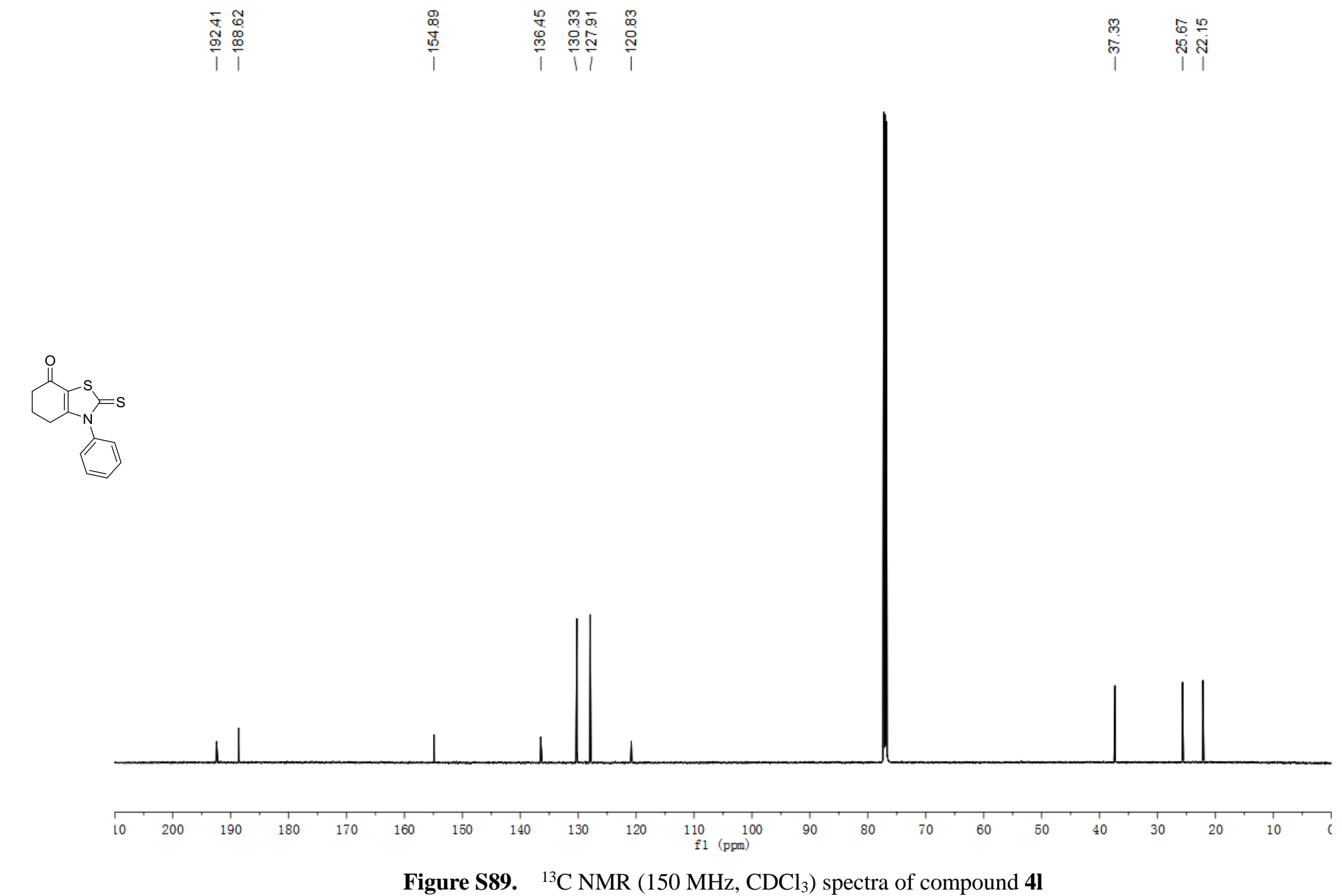



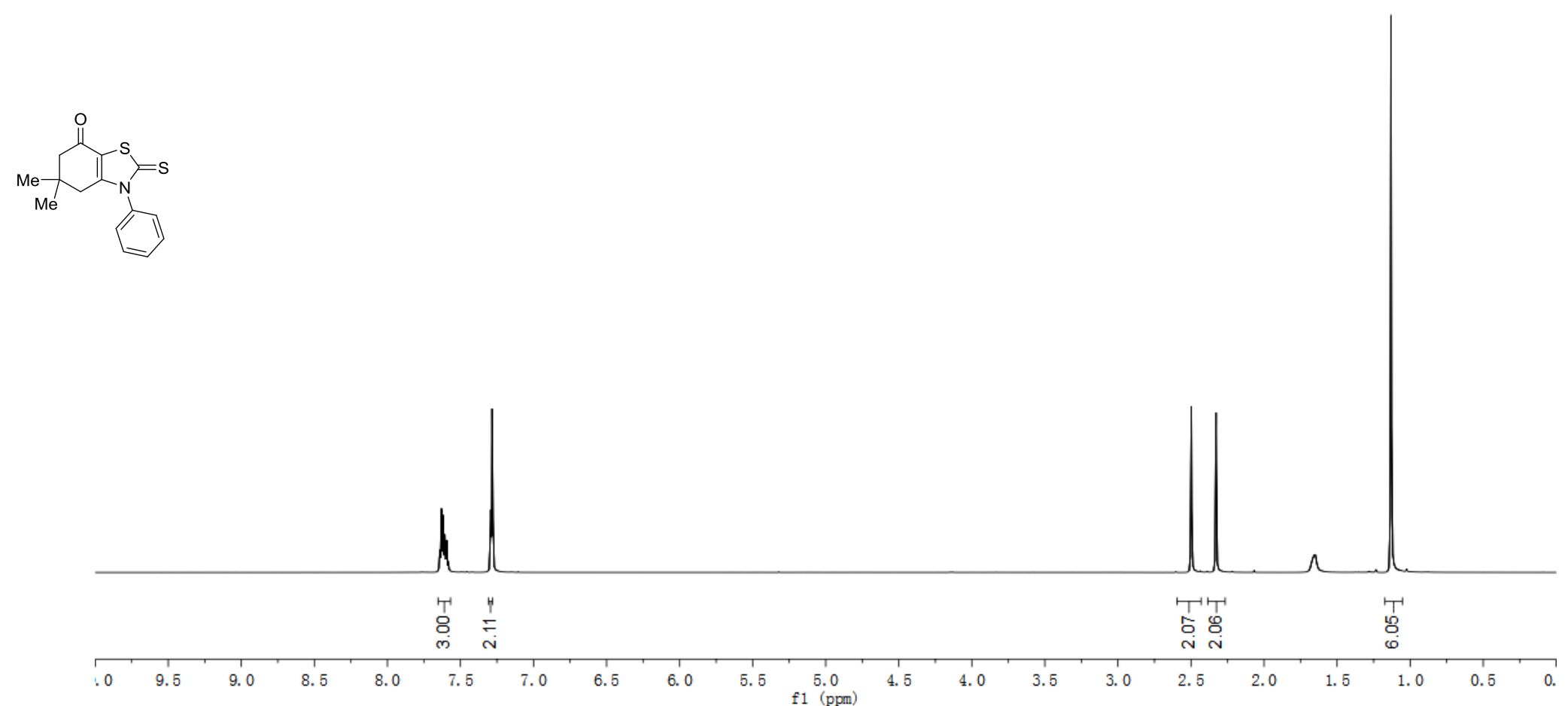

Figure S90. ${ }^{1} \mathrm{H} \mathrm{NMR}\left(600 \mathrm{MHz}, \mathrm{CDCl}_{3}\right)$ spectra of compound $\mathbf{4 m}$ 


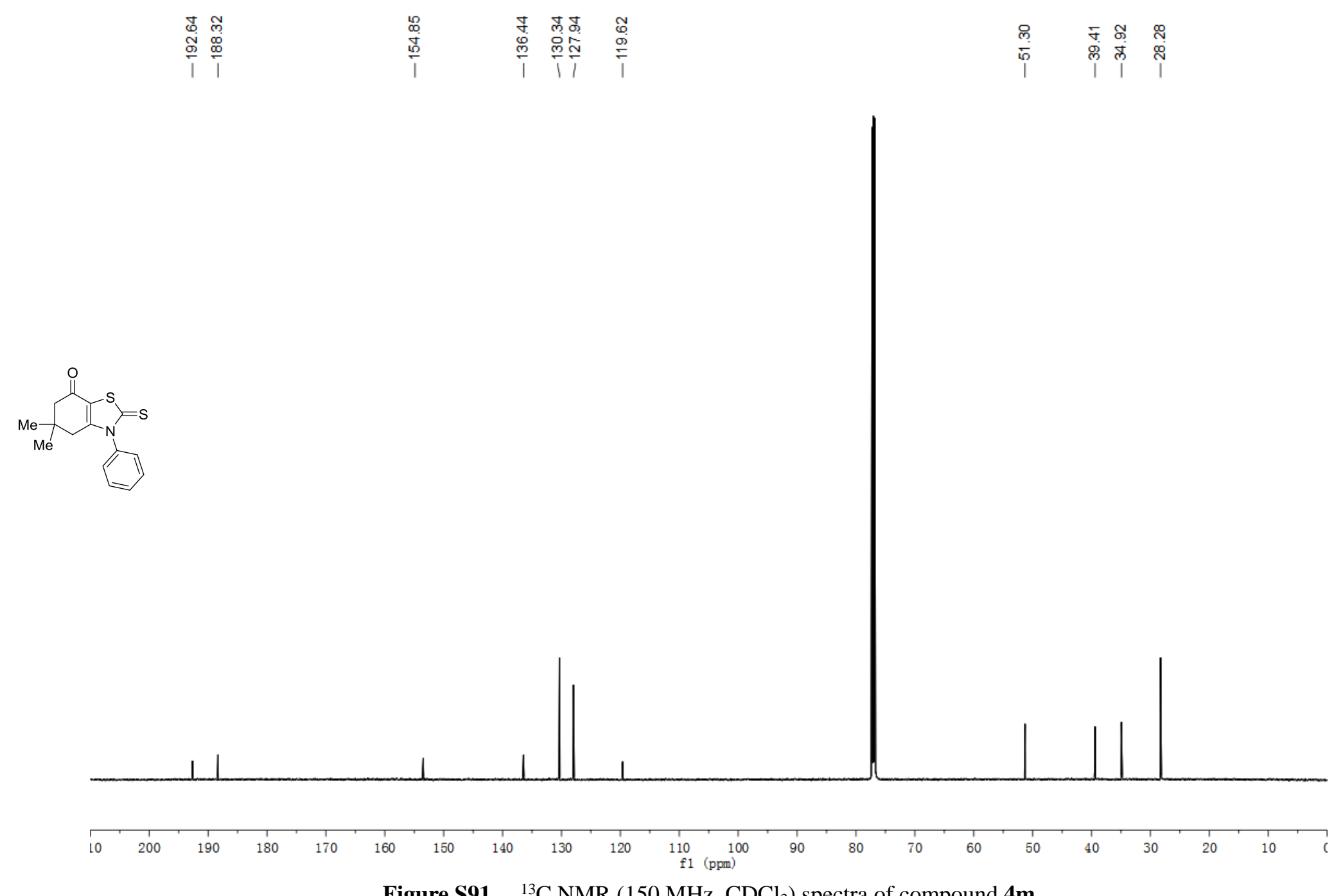

Figure S91. ${ }^{13} \mathrm{C} \mathrm{NMR}\left(150 \mathrm{MHz}, \mathrm{CDCl}_{3}\right)$ spectra of compound $\mathbf{4 m}$ 

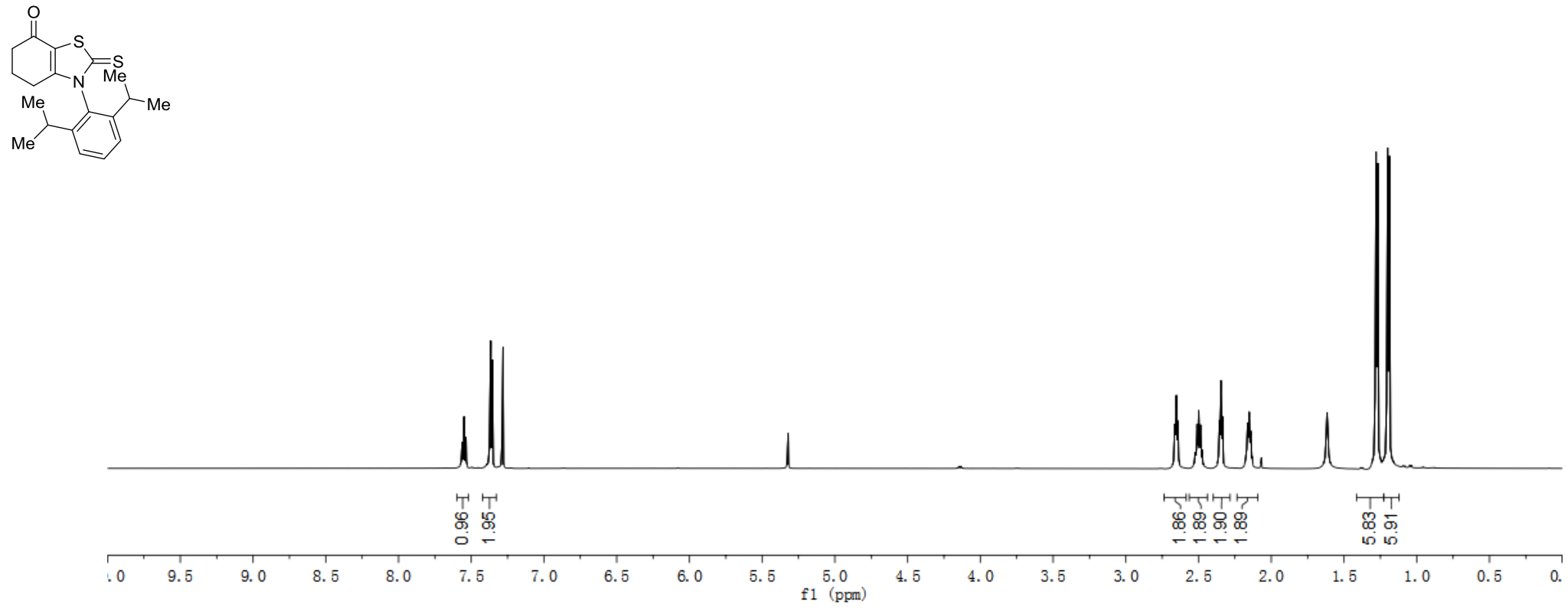

Figure S92. $\quad{ }^{1} \mathrm{H} \mathrm{NMR}\left(600 \mathrm{MHz}, \mathrm{CDCl}_{3}\right)$ spectra of compound $4 \mathbf{n}$ 

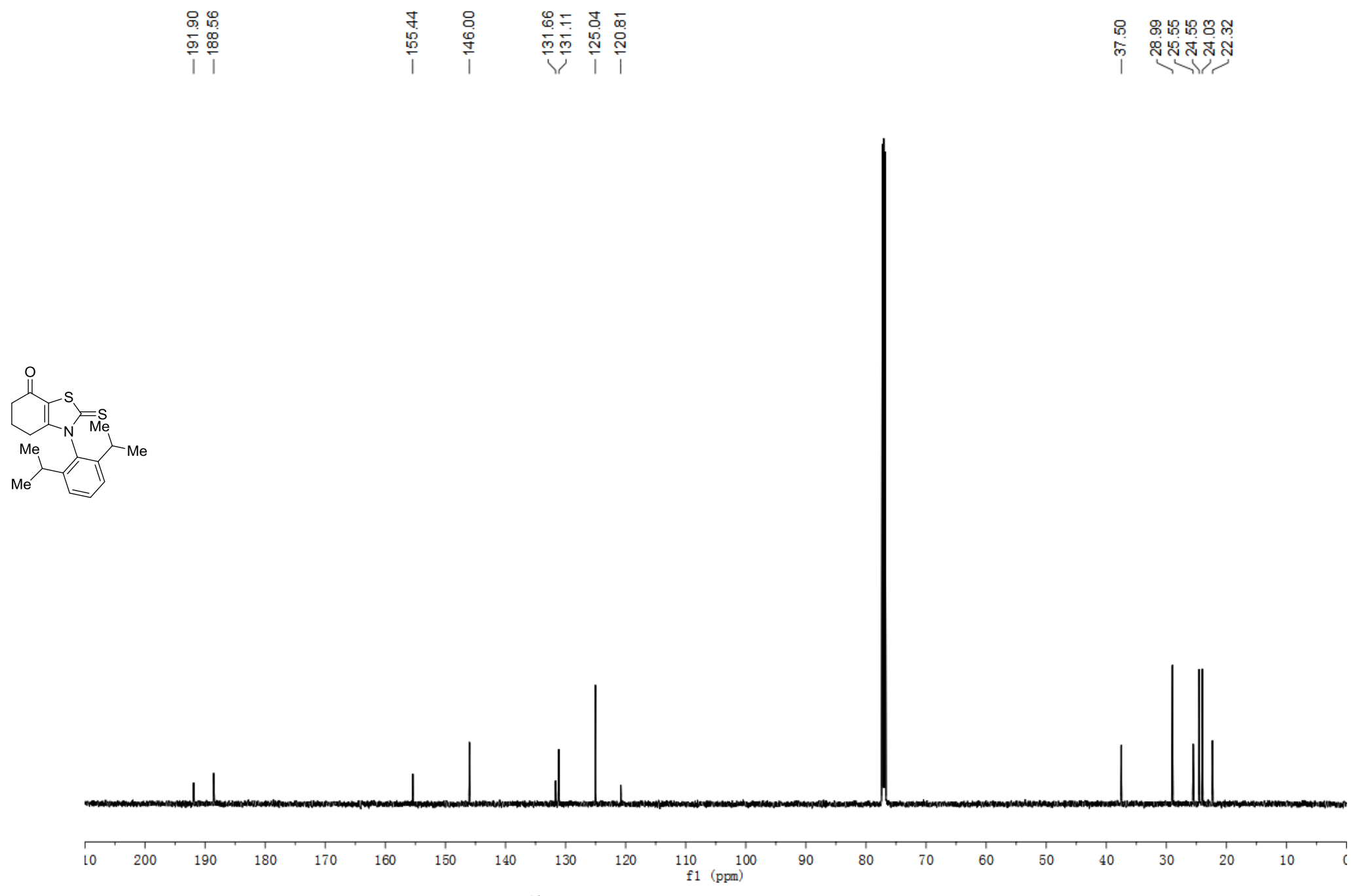

Figure S93. ${ }^{13} \mathrm{C} \mathrm{NMR}\left(150 \mathrm{MHz}, \mathrm{CDCl}_{3}\right)$ spectra of compound $4 \mathbf{n}$ 

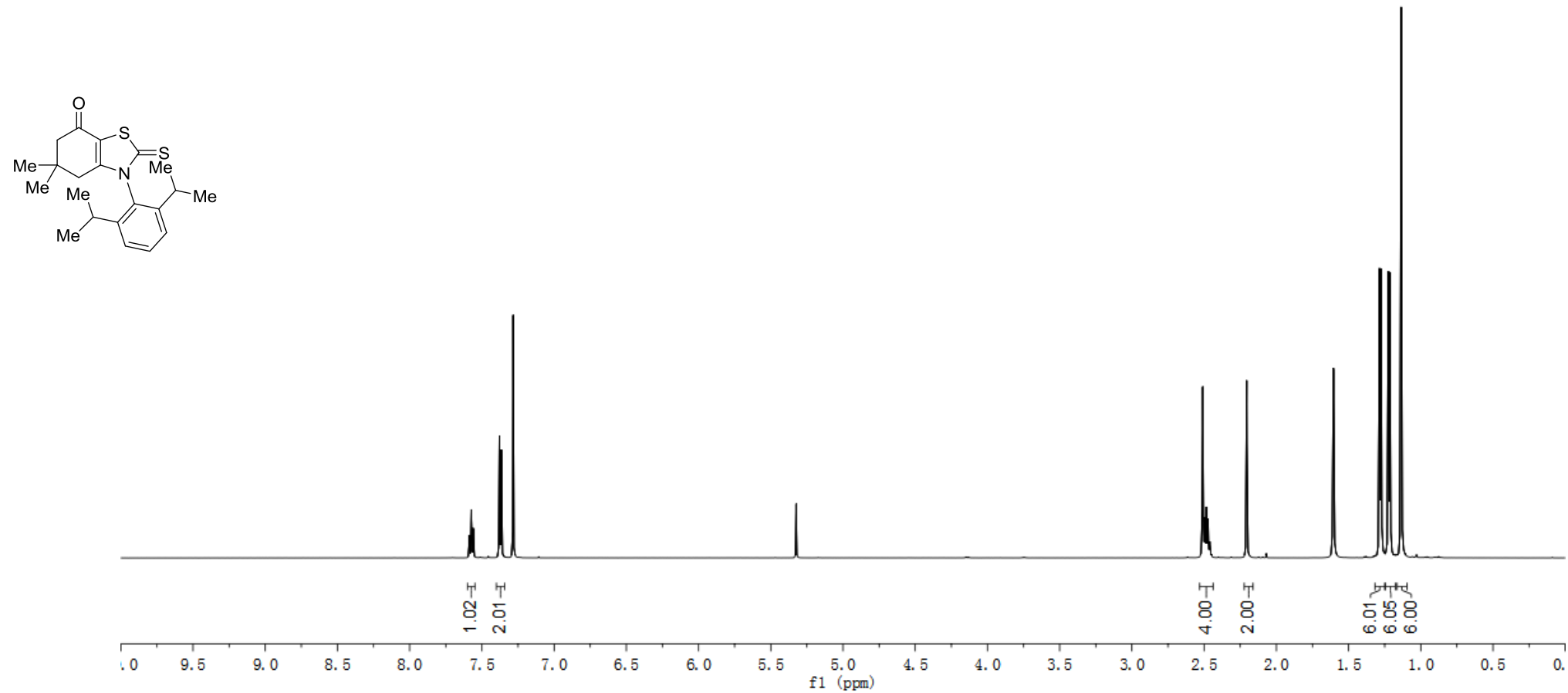

Figure S94. $\quad{ }^{1} \mathrm{H} \mathrm{NMR}\left(600 \mathrm{MHz}, \mathrm{CDCl}_{3}\right)$ spectra of compound 40 


$$
=1
$$




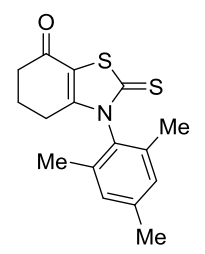




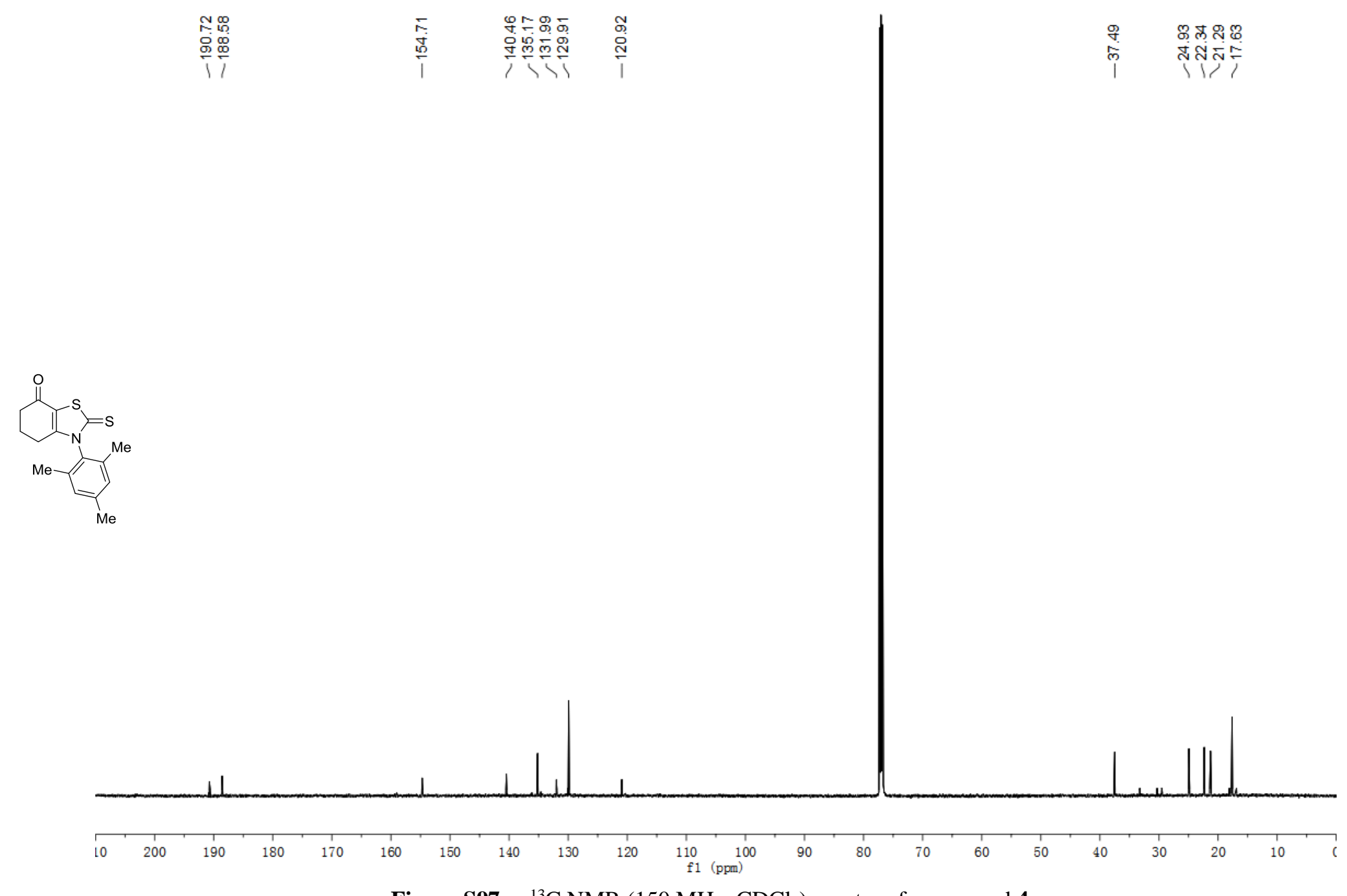

Figure S97. ${ }^{13} \mathrm{C} \mathrm{NMR}\left(150 \mathrm{MHz}, \mathrm{CDCl}_{3}\right)$ spectra of compound $\mathbf{4 p}$ 

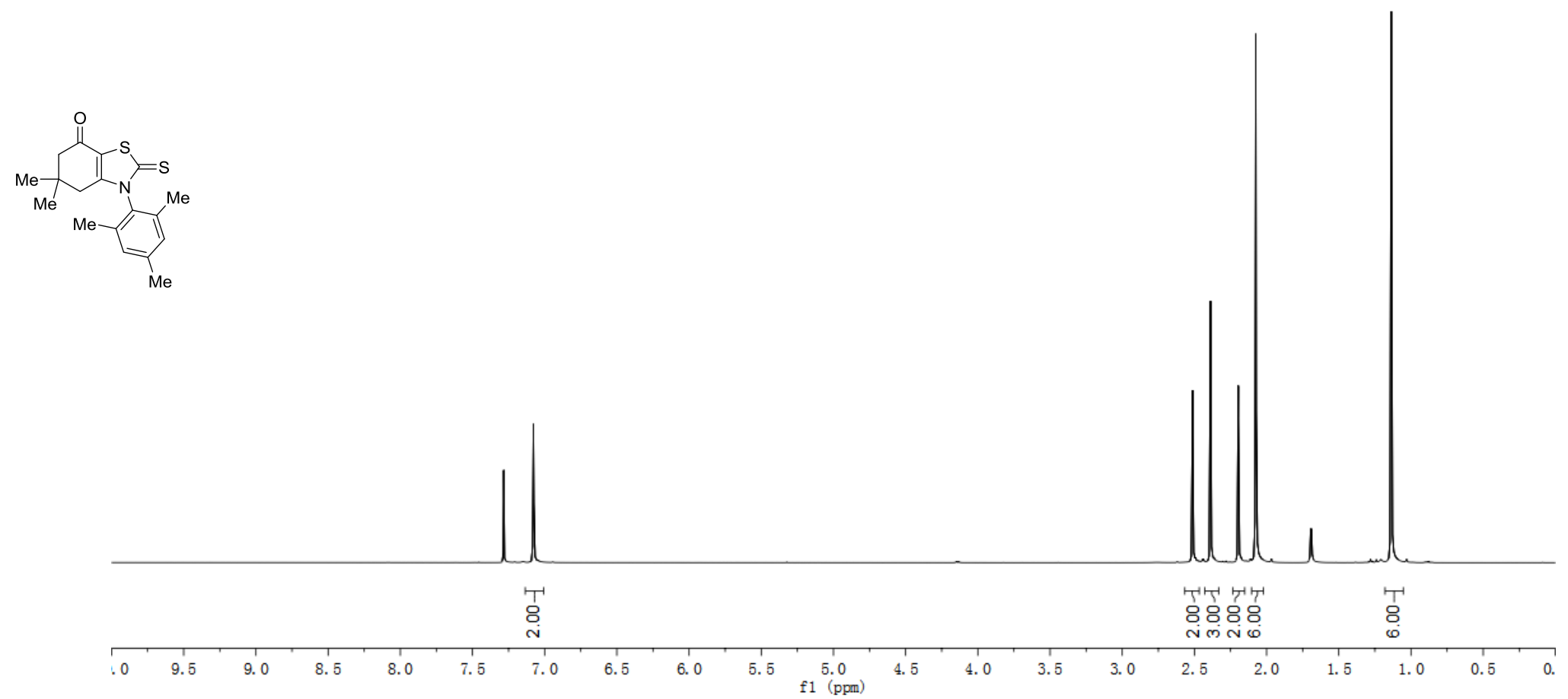

Figure S98. $\quad{ }^{1} \mathrm{H} \mathrm{NMR}\left(600 \mathrm{MHz}, \mathrm{CDCl}_{3}\right)$ spectra of compound $\mathbf{4 q}$ 


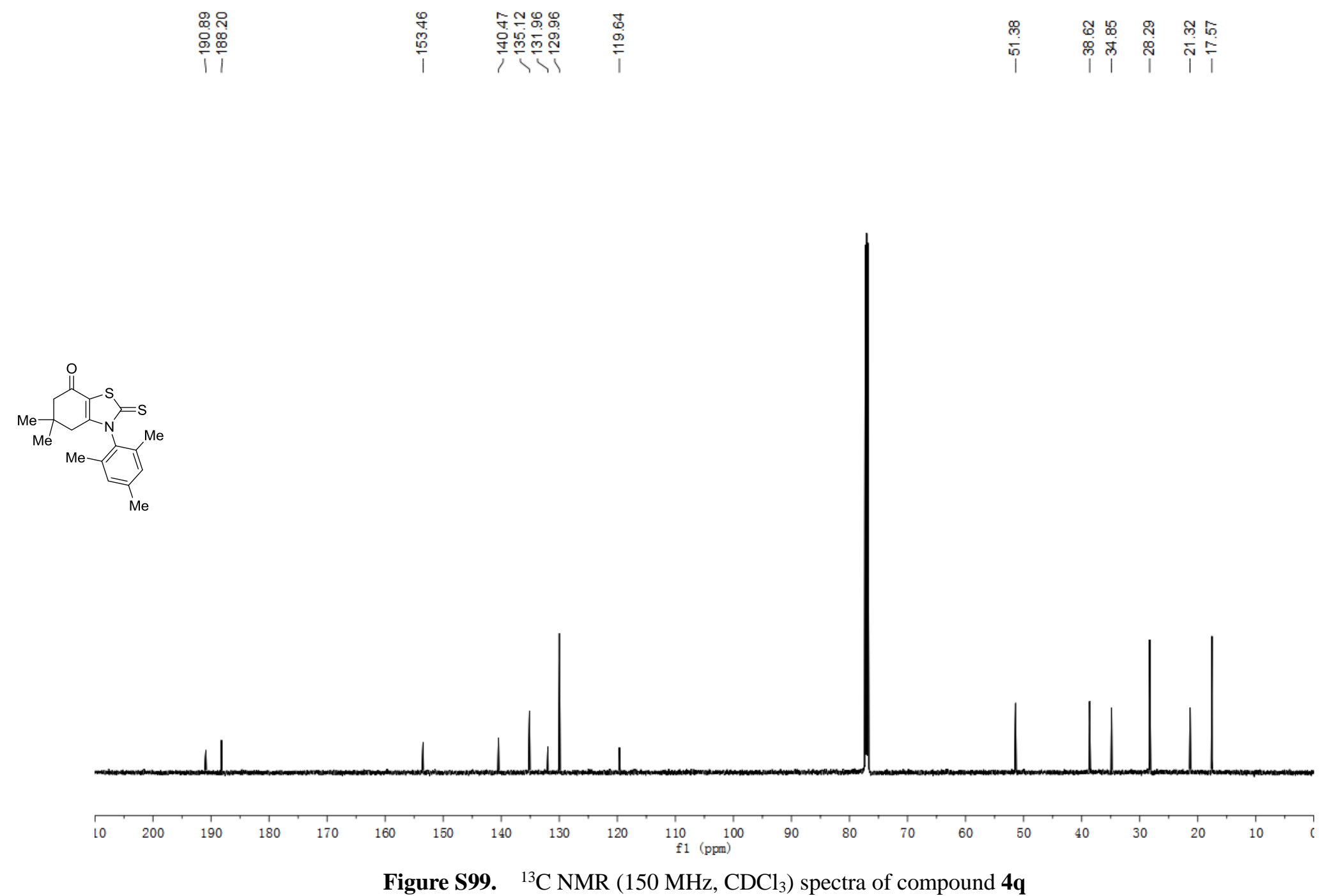


$\overbrace{N}^{S}=s$

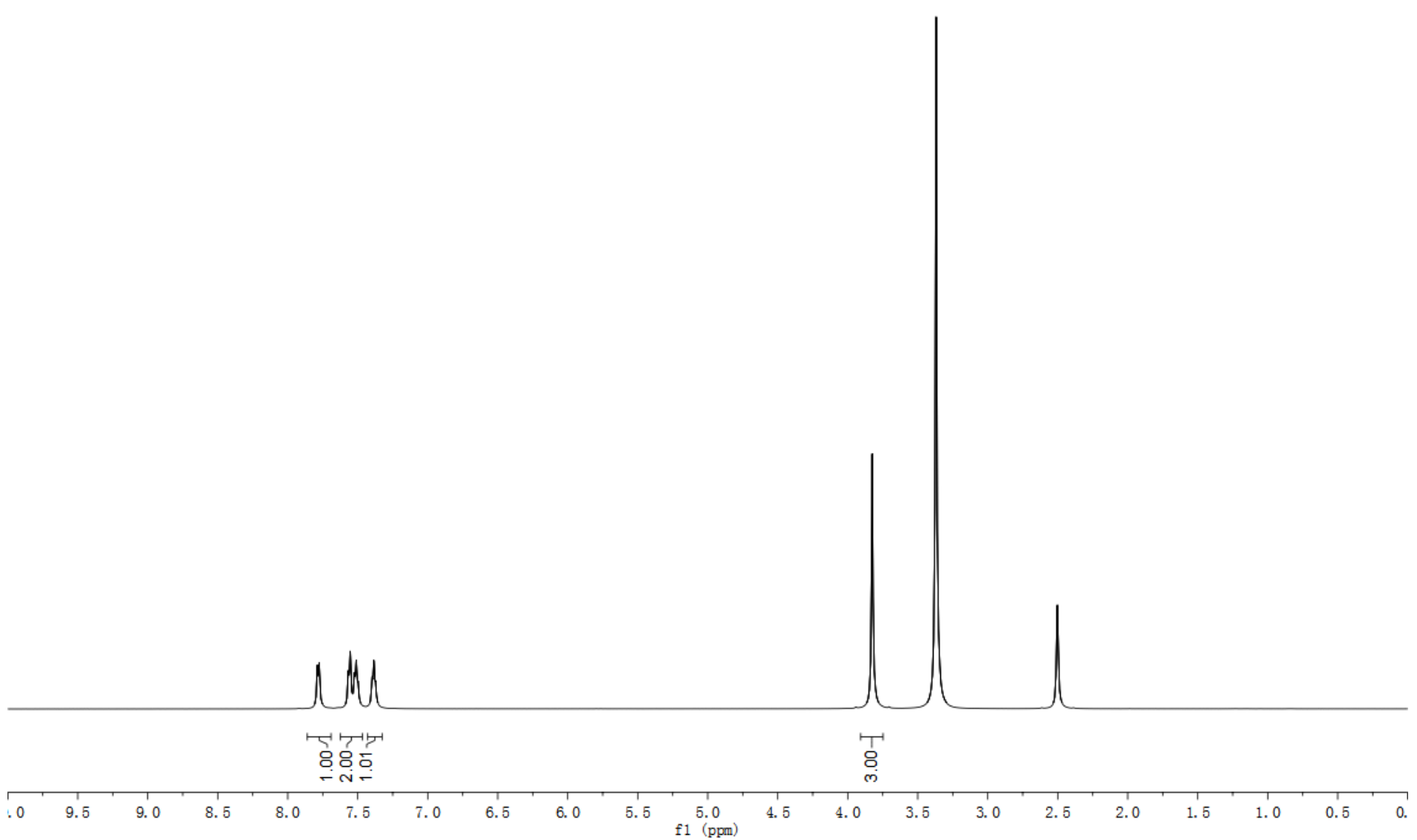

Figure S100. $\quad{ }^{1} \mathrm{H}$ NMR $\left(600 \mathrm{MHz}, \mathrm{DMSO}-d_{6}\right)$ spectra of compound 7a 
$\underset{\substack{5 \\ \infty \\ \infty}}{\frac{5}{1}}$

i
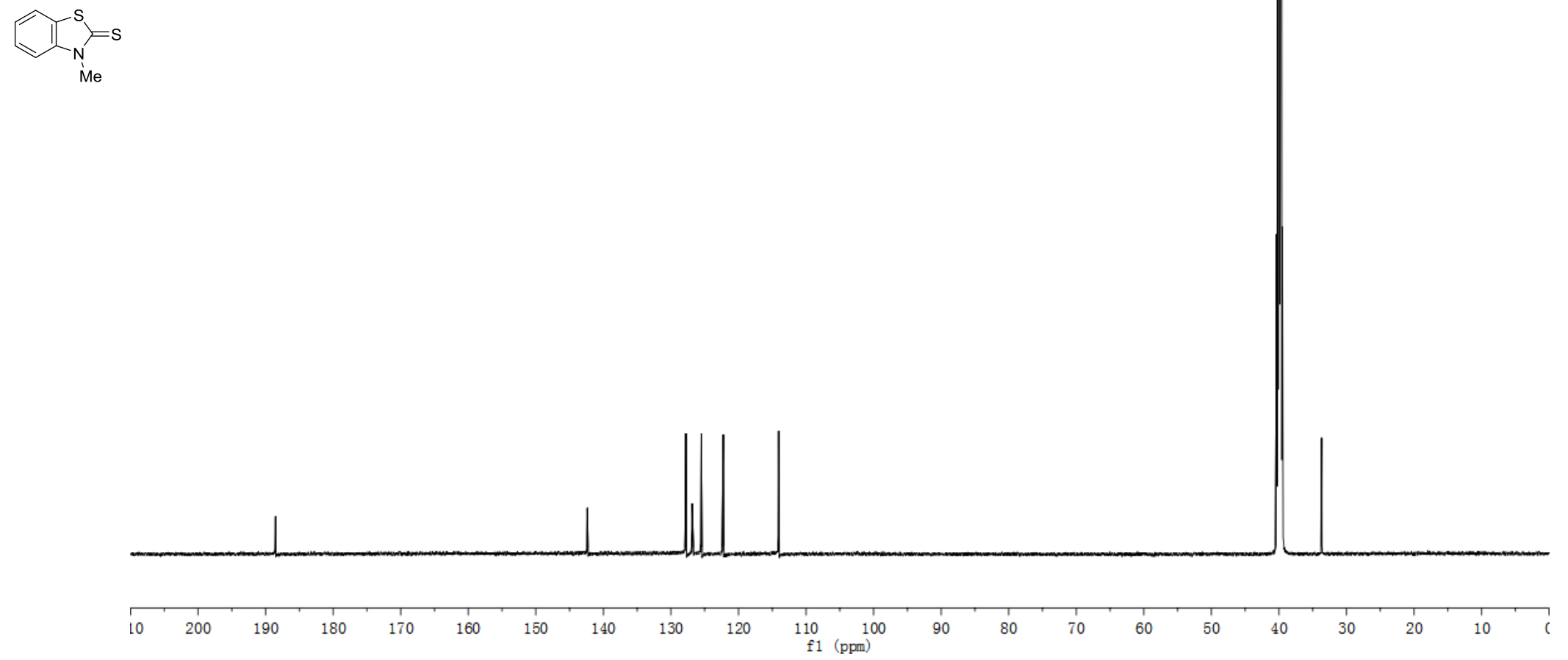

Figure S101. ${ }^{13} \mathrm{C}$ NMR $\left(150 \mathrm{MHz}, \mathrm{DMSO}-d_{6}\right)$ spectra of compound Ta

S121 


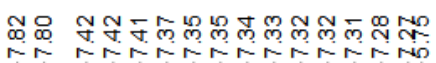

${ }_{N}^{s}=\mathrm{s}$

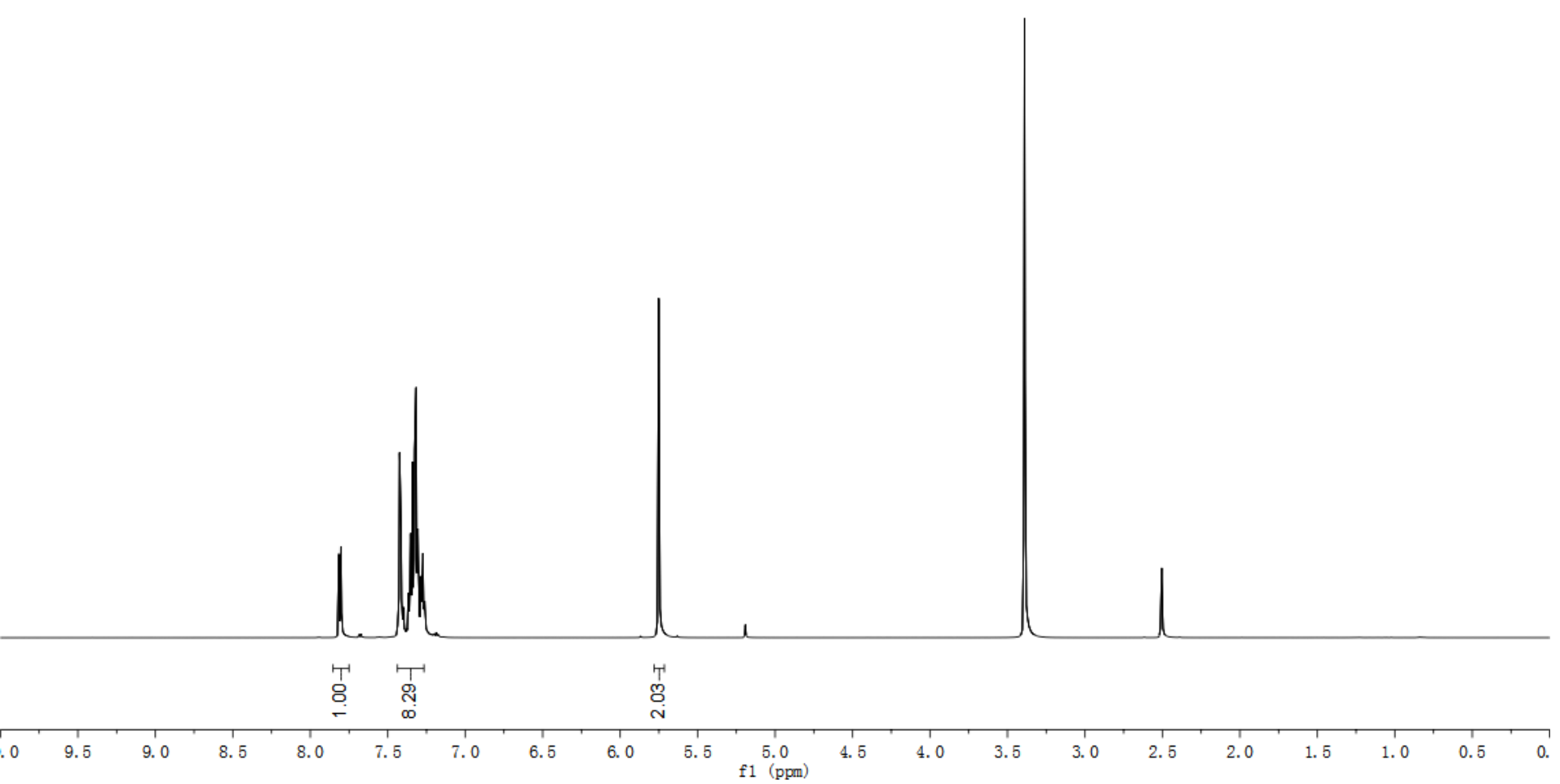

Figure S102. ${ }^{1} \mathrm{H}$ NMR (600 MHz, DMSO- $\left.d_{6}\right)$ spectra of compound $7 \mathbf{b}$ 


$$
1
$$




\section{References and notes.}

1. (a) Tingoli, M.; Mazzella, M.; Panunzi, B.; Tuzi, A. L-Proline-catalyzed activation of methyl ketones or active methylene compounds and DMF-DMA for syntheses of (2E)-3-dimethylamino-2-propen-1-ones. Eur. J. Org. Chem. 2011, 399. (b) Das, B.; enkateswarlu, K.; Majhi, V.; Reddy, M. R.; Reddy, K. N.; Yerra Rao, K.; Ravikumar, K.; Sridhar, B. Highly efficient, mild and chemo- and stereoselective synthesis of enaminones and enamino esters using silica supported perchloric acid under solvent-free conditions. J. Mol. Catal. A: Chem. 2006, 246, 276. (c) Liu, Y.; Zhou, R. Wan, J.-P. Water-promoted synthesis of enaminones. Mechanism investigation and application in multicomponent reactions. Synth. Commun. 2013, 43, 2475.

2. Gao, Y.; Liu, Y.; Wei, L.; Wan, J. Synthesis of enaminones containing diverse $N, N$-disubstitution via simple transamination: a study with sustainable catalyst-free operation. Res. Chem. Intermed. 2017, 43, 5547.

3. (a) Dang, P.; Zeng, W.; Liang,Y. Copper-catalyzed three-component synthesis of benzothiazolethiones from $o$-iodoanilines, isocyanide, and potassium sulfide. Org. Lett. 2015, 17, 34. (b) Tan, W.; Wang, C.; Jiang, X. Green carbon disulfide surrogate via a combination of potassium sulfide and chloroform for benzothiazine-thione and benzothiazolethione construction. Org. Chem. Front. 2018, 5, 2390.

4. CCDC 2062625 contain the supplementary crystallographic data for compound 2a. These data can be obtained free of charge from The Cambridge Crystallographic Data Center via www.ccdc.cam.ac.uk/data_request/cif. 